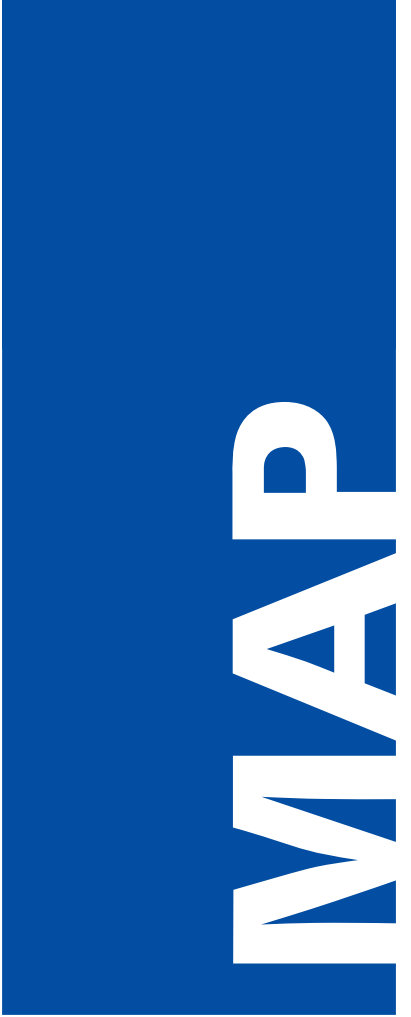

MULTI ACTORS AND MULTI MEASURES PROGRAMMES

\title{
STRATA
}
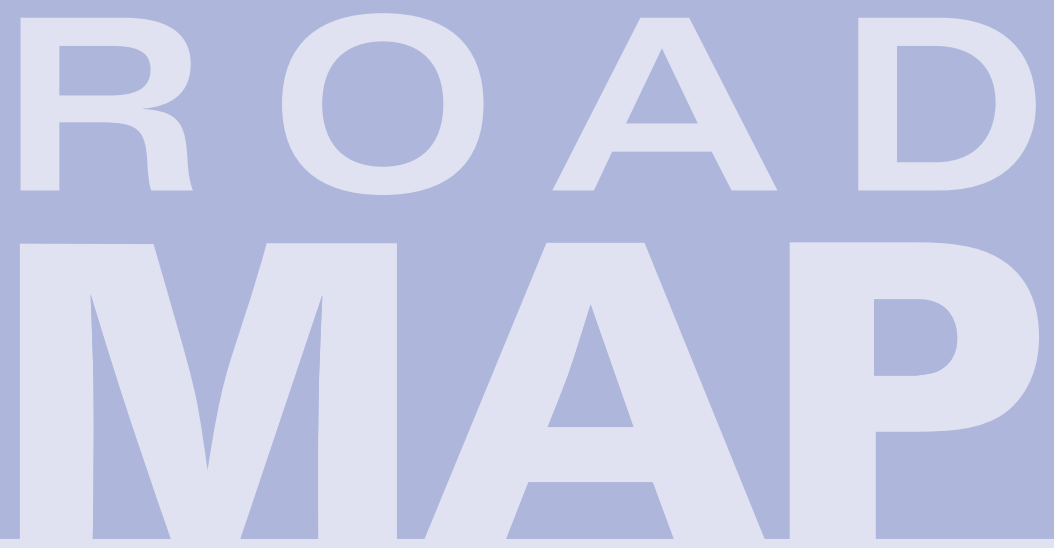

\section{Good practices} for the management of Multi Actors and Multi Measures Programmes (MAPs) in RTDI policy

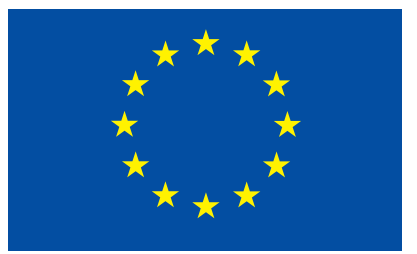


Good practices for the management

of Multi Actors and Multi Measures

Programmes (MAPs) in RTDI policy

"It's your mind that creates the world" (Buddha)

MAP-TN started three years ago out of a discussion, and that's how the network went on. Exchange of experience, intensive work and sharing of knowledge produced this guide to MAPs - our roadMAP.

It may not be best practice - but in a world of humans, different backgrounds and minds that will never be achieved - but good practice it is. We hope to give you an insight in the work with and some guidance to MAPs.

A big THANK YOU to the numerous people, who joined the network, gave their input, time or support - or just stuck around when the road seemed to go off its tracks...

The MAP-TN Team

"It's the MAP that creates your road" (The MAP-TN Team) 


\section{CONTENT}

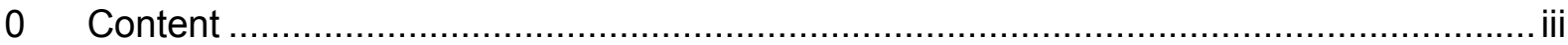

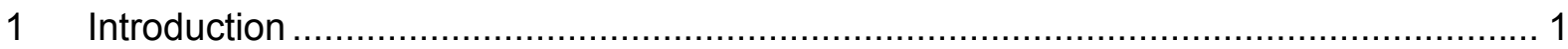

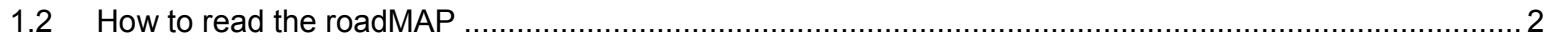

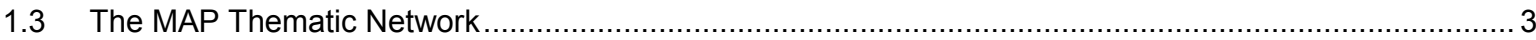

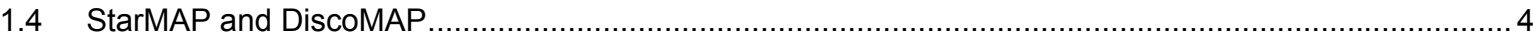

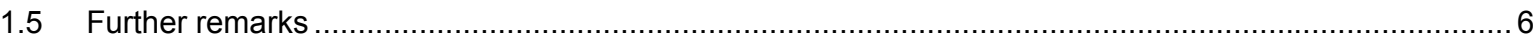

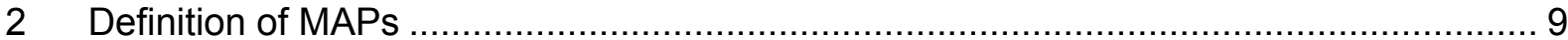

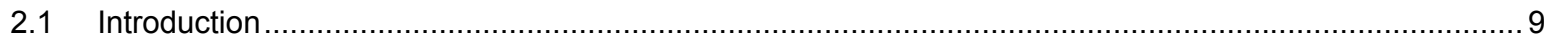

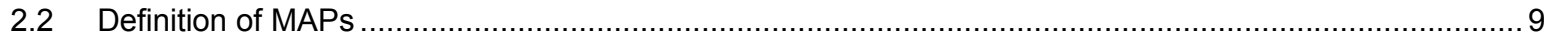

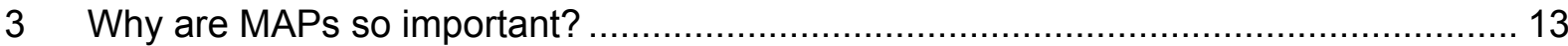

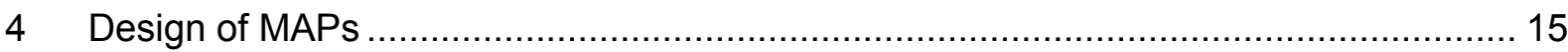

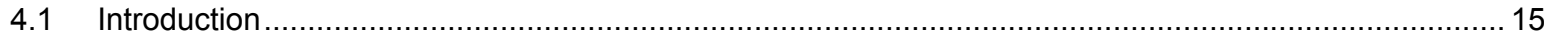

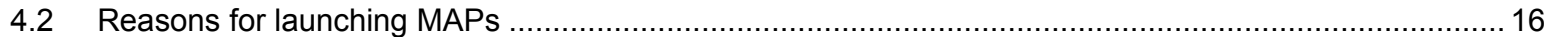

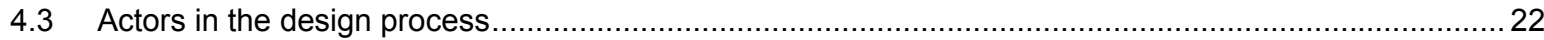

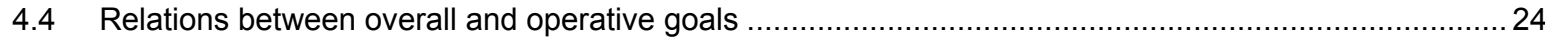

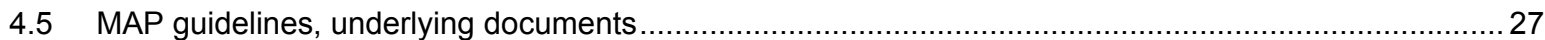

4.6 International influence and opening up of national programmes ..................................................... 31

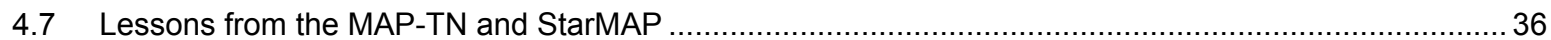

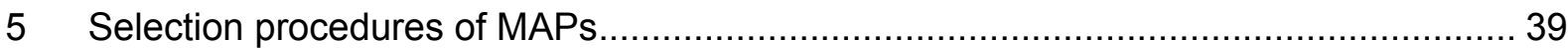

5.1 Introduction

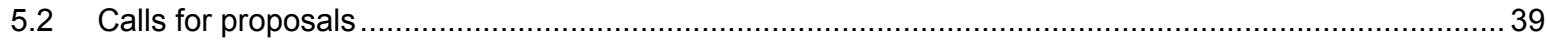

5.3 Proposal evaluation - particular challenges; the systemic view .........................................................51

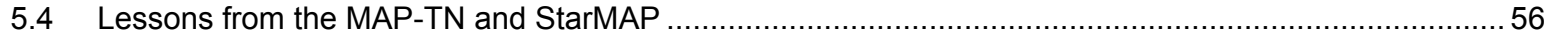

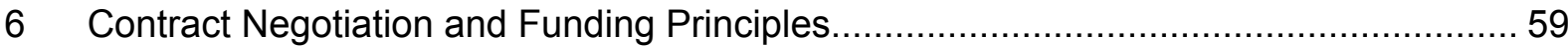

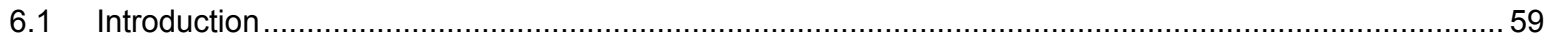

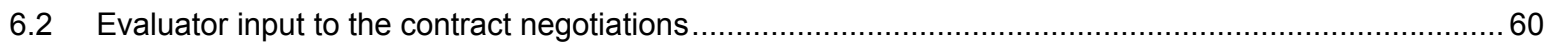

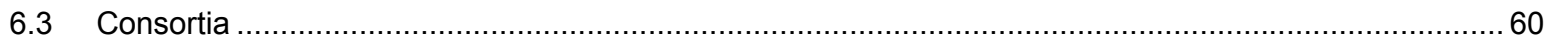

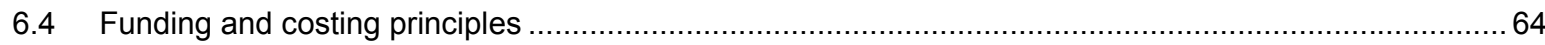

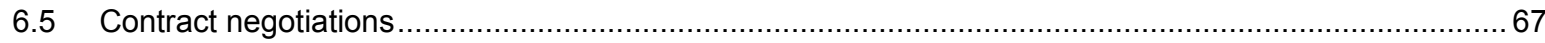

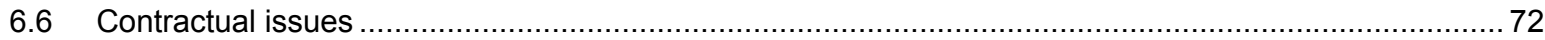

6.7 Concluding note 


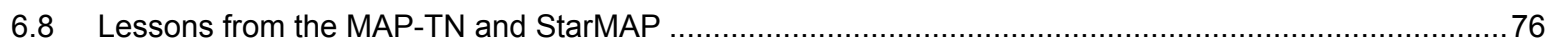

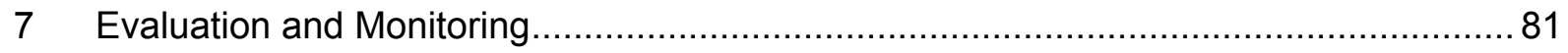

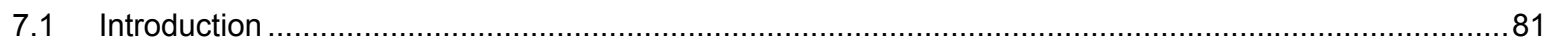

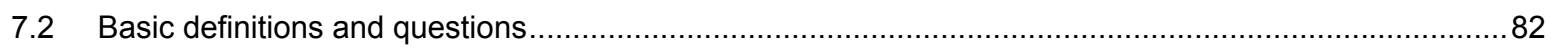

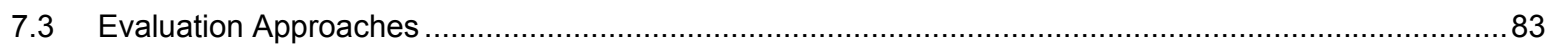

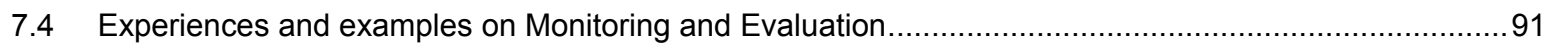

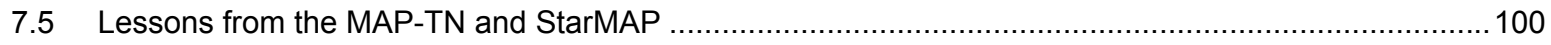

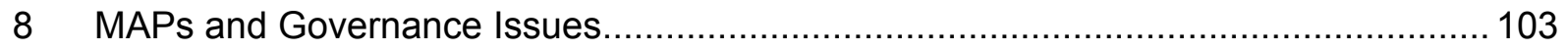

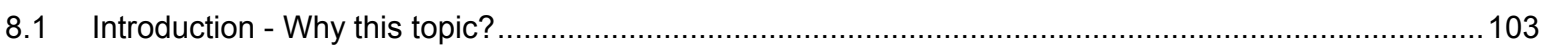

8.2 The terms „governance" and "social norms": difficult approaches ........................................... 104

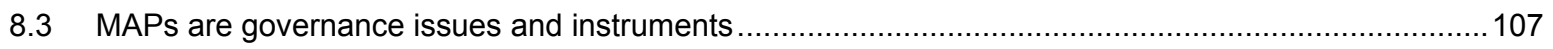

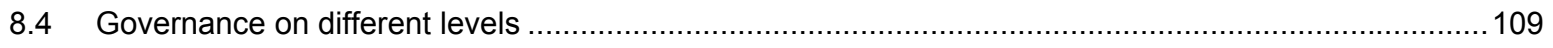

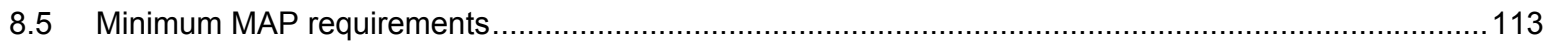

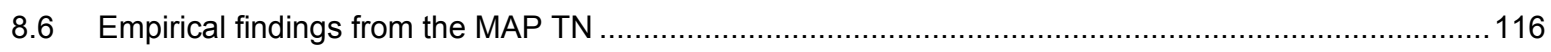

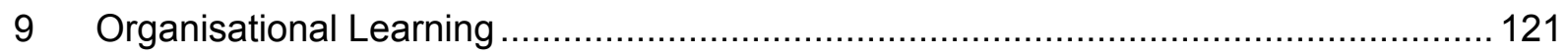

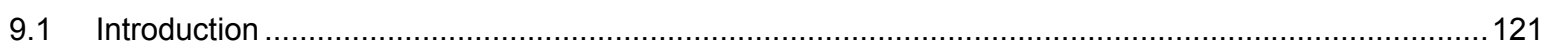

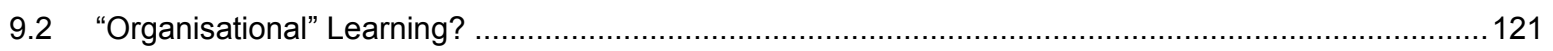

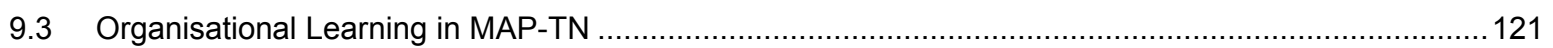

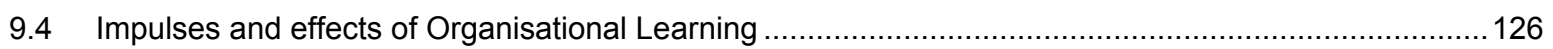

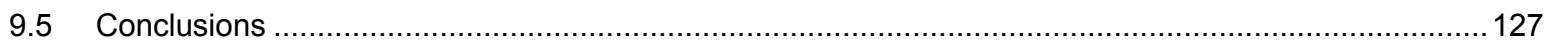

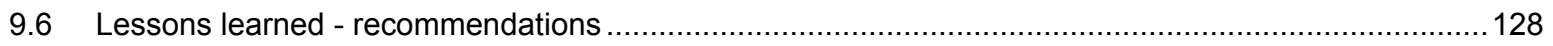

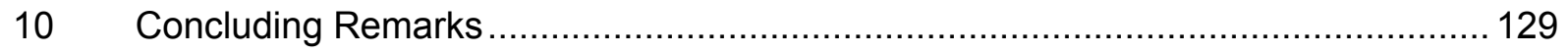

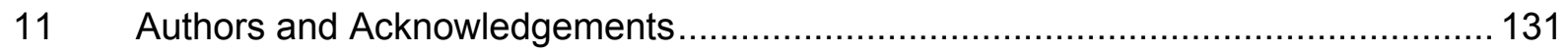

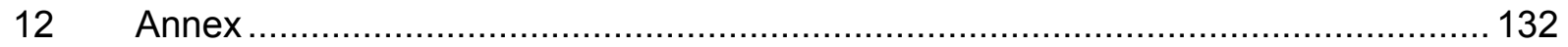

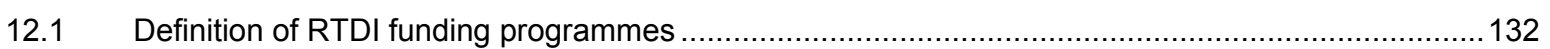

12.2 Competence Centre Programmes and Network oriented Programmes .......................................135

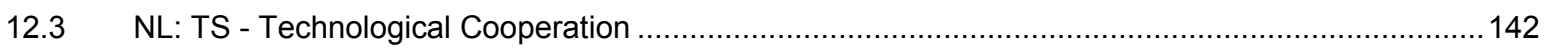

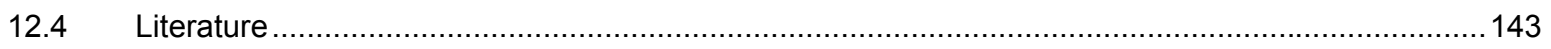

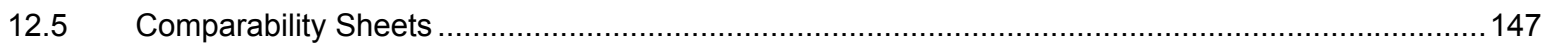




\section{INTRODUCTION}

This handbook is the result of a two years information exchange amongst experienced RTDI ${ }^{1}$ programme managers and complementary organisations. It tries to codify good practices that were observed in 16 RTDI programmes. 10 programmes were discussed in depth in workshops of the MAP Thematic Network (MAP-TN), 6 other programmes were studied within the STRATA project StarMAP (both projects will be described in chapter 1.4).

The RTDI programmes selected may be classified as Multi Actor Multi Measure programmes (MAPs; further description see chapter 4) with a focus on cooperation between science and industry. Nevertheless a huge variety of features and management practices was observed, depending both on the National Innovation System (NIS) in the respective country (especially the institutional setting) and on the problems addressed. However there are communalities that turned out to be used widely and successfully within the limits of certain frames and conditions. As these frames and conditions are the key issue for the successful implementation of an RTDI programme, the following approach was chosen for this handbook:

\subsubsection{Content of the handbook:}

At the very beginning you will find a definition of MAPs, showing the complexity of this specific type of programme to which we refer specifically in this handbook which was developed by the network. The MAP definition is followed by introductory remarks about "why are MAPs so important?"

After that you will note that the chapters follow the life cycle of RTDI funding programmes, namely design, selection procedures and contract negotiations, implementation, monitoring and evaluation as shown in the graph below:

Exhibit 1: $\quad$ Policy Cycle for RTDI Programmes

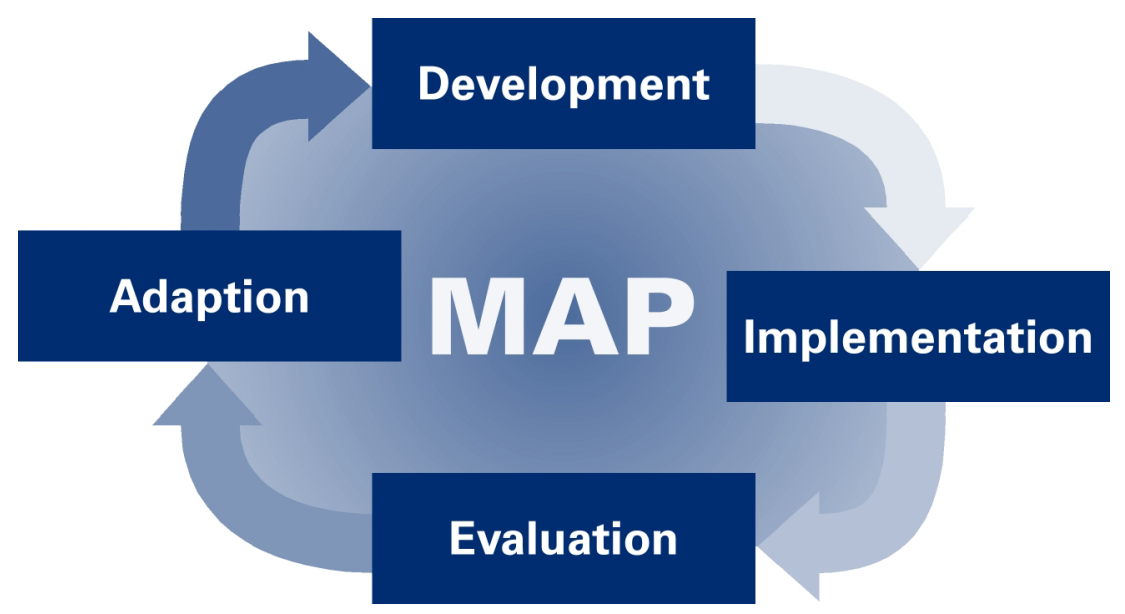

${ }^{1}$ Research, Technological Development and Innovation 
The chapter on the design phase deals with the reasons for launching a MAP, actors in the design process, the relations between overall and operative goals and the interface between programme design and evaluation, MAP guidelines, international influence and opening up of national programmes. Finally we talk about the lessons learned from the MAP-TN and StarMAP.

The following chapter describes strategies and operative issues with regard to selection procedures, e.g. amongst which options you can chose and which advantages and disadvantages go along with a specific option. We talk about calls for proposals and proposal evaluation, followed by lessons learned from the individual MAPs.

We continue with contract negotiations, having a look at the evaluator's input to the contract negotiations, consortia building, funding and costing principles, the contract negotiations themselves and finally contractual issues, again with a number of lessons learned.

The last chapter is about monitoring and evaluation, looking at particularities of MAP evaluations, impact measurement approaches and the usefulness of measuring MAPs. The chapter concludes with numerous examples and experiences from MAP-TN and StarMAP on monitoring, project evaluation and programme evaluation.

The latter is a general feature for all chapters mentioned above: All chapters include examples and experiences from 16 RTDI programmes, $15^{2}$ of which could be classified as MAPs according to the definition developed in the MAP-TN (see chapter 2.2).

In the final part of the handbook you find a chapter on governance and social norms, touching the issues of power, steering and framework conditions as well as funding cultures (traditions, experiences and habits) and a chapter about Organisational Learning.

In the Annex we have also attached comparability sheets of the main features of the MAP-TN programmes, accompanied by detailed programme descriptions, as well as comparability sheets from the StarMAP programmes.

\subsection{How to read the roadMAP}

The target groups for our handbook are RTDI programme managers, RTDI policy makers and evaluators who would like to get a better insight into good internationally proven management practices of RTDI funding programmes as MAPs.

Some of the content might sound trivial. In fact it is not, if you consider the complexity, the actors settings and all the things that can go wrong, which were also openly discussed in our network. The lessons learned also include critical success factors described in this handbook.

\footnotetext{
${ }^{2}$ The exception is the TEFT programme in Norway, which is according to our definition not considered a MAP.
} 
Please note that this handbook is the "long" version consisting of a lot of background material and references and in particular a lot of practical hints whereas the "short" version concentrates on the latter. Please keep in mind that mainly programme managers were involved in the preparation of this handbook and that is why it is very practically oriented rather than being a scientific paper.

Please note further that references are only used for the more theoretical issues in this handbook, for the practical part stemming from MAP-TN and StarMAP we have deliberately not quoted references for reasons of brevity. A short description of MAP-TN and StarMAP programmes can be found in the Annex. Further detailed background material referring to all issues discussed in this handbook can be downloaded from the MAP-TN homepage (www.map-network.net) which also serves as a forum for feedback and discussion.

The members of the MAP Thematic Network very much hope that you find this handbook useful for your daily work, either to be trained (e.g. for newly recruited programme managers) or to have your way of working and your management methods confirmed or modified.

\subsection{The MAP Thematic Network}

In 2000 TIG, an Austrian agency specialised in the management of MAPs started to create a network of RTDI programme managers and complementary organisations to exchange experience and know-how about the strategic and day-to-day-work of designing, implementing and evaluating RTDI funding programmes. These ideas mainly drew on the results of a workshop in Karlsruhe about the challenges of Multi Actors - Multy Measures Programmes at ISI in $2000^{3}$. A proposal - called MAP-TN - was therefore submitted in June 2001 to the STRATA programme $\left(5^{\text {th }}\right.$ EU Framework Programme) and started in January 2002 with the following partners working together over a period of two years:

- Austria: TIG - Technologie Impulse Gesellschaft (www.tig.or.at)

- Germany: ISI - Fraunhofer Institute for Systems and Innovation Research (www.isi.fhg.de)

- Spain / Basque Country: Asociación Unitec (www.asociacionunitec.com)

- Sweden: VINNOVA - The Swedish Agency for Innovation Systems (www.vinnova.se)

- United Kingdom: Technopolis Ldt. (www.technopolis-group.com)

- Hungary: Ministry of Education / Research and Development Division (www.om.hu)

- Slovenia: PMIS-lab - Faculty of Mechanical Engineering, University of Maribor (www.uni-mb.si)

- Ireland: SEI - Sustainable Energy Ireland (www.sei.ie)

- Belgium / Flanders: IWT - Institute for the Promotion of Innovation by Science and Technology in Flanders (www.iwt.be)

${ }^{3}$ see the contribution of Bührer, Kuhlmann and Stampferin Bührer / Kuhlmann 2003 
- Estonia: Ministry of Economic Affairs and Communications (www.mkm.ee)

- EARMA - the European Association for Research Managers and Administrators (no RTDI programme involved; partner for dissemination activities) (www.earma.org)

\subsection{StarMAP and DiscoMAP}

At the very moment when the network was granted EU funding two further proposals were launched within the STRATA programme to ensure funding of additional activities with regard to "good practices in MAP management". The projects "StarMAP" and "DiscoMAP". Also these proposals were evaluated positively. With StarMAP the "database" of the network was expanded as the project looked at six other MAPs in the Netherlands, France, Finland, Norway ${ }^{4}$, Canada and Australia, focussing on quite long term mature programmes in countries not participating in the MAP-TN. DiscoMAP increased the dissemination activities, respectively an international conference at the end of the projects, scheduled for the 29 and 30 March 2004 in Vienna.

\subsubsection{Which MAPs were investigated?}

The MAPs under comparison can be classified as following:

Exhibit 2 Characteristics of Network or Cluster oriented programmes and Competence Centre programmes

\begin{tabular}{|l|l|}
\hline $\begin{array}{l}\text { Competence Centre programmes } \\
\text { (with or without thematic focus) }\end{array}$ & $\begin{array}{l}\text { Network or Cluster oriented programmes } \\
\text { (with or without thematic focus; some are SME oriented } \\
\text { programmes with a rather loose network character) }\end{array}$ \\
\hline $\begin{array}{l}\text { Very strong cooperation within the projects (centre) } \\
\text { "Physical" centres }\end{array}$ & $\begin{array}{l}\text { Loose cooperation within the projects } \\
\text { Science-driven }\end{array}$ \\
\hline
\end{tabular}

Note that although in some cases Competence Centres have to be created as a legal entity of their own, in our opinion they are not seen as classical institutions for three reasons: (i) in general, institutions aim at a longer term duration (with no ex ante fixed date of termination in mind) and thus are associated with financing instruments that are in general not to be terminated after a defined period of time (e.g. General University Funds) Competence Centres have a fixed end date and are the "deliverables" of funding programmes; (ii) in Competence Centres, network characteristics e.g. non-hierarchical formal structures and cooperation are dominant; (iii) the centres are always public private partnerships regarding financing, planning and operation.

${ }^{4}$ It has to be pointed out that the Norwegian programme TEFT turned out (after an in-depth review) to be MAP-like but doesn't fulfil all MAP criteria, mentioned in chapter 2. However, as there might be some interesting aspects of the SME targeted programme, we left it in the descriptions. 
An overview of programmes and their classification is presented in the following table and graph:

Exhibit 3: Programme overview

\begin{tabular}{|c|c|c|c|}
\hline Country & & Name of the MAP & Classification of MAP \\
\hline Austria & AT & $\begin{array}{l}\text { Kplus } \\
\text { www.kplus.at }\end{array}$ & Competence Centre programme \\
\hline Australia & $\mathrm{AU}$ & $\begin{array}{l}\text { CRC } \\
\text { www.crc.gov.au }\end{array}$ & Competence Centre programme \\
\hline Belgium & $\mathrm{BE}$ & $\begin{array}{l}\text { VIS / CIN } \\
\text { www.iwt.be }\end{array}$ & Network oriented programme \\
\hline Canada & $\mathrm{CA}$ & $\begin{array}{l}\text { NCE } \\
\text { www.nce.gc.ca }\end{array}$ & Network oriented programme \\
\hline Germany & $\mathrm{DE}$ & $\begin{array}{l}\mathrm{CCN} \\
\text { www.nanonet.de }\end{array}$ & $\begin{array}{l}\text { Competence Centre programme } \\
\text { with thematic focus (nanotechnology) }\end{array}$ \\
\hline Estonia & $\mathrm{EE}$ & $\begin{array}{l}\text { Competence Centres Estonia } \\
\underline{w w w . m k m . e e}\end{array}$ & Competence Centre programme \\
\hline Spain & ES & $\begin{array}{l}\text { PCTI-Cooperación } \\
\text { www.euskadi.net/industria }\end{array}$ & $\begin{array}{l}\text { Network oriented programme } \\
\text { (with SME focus) }\end{array}$ \\
\hline Finland & $\mathrm{FI}$ & $\begin{array}{l}\text { Technology Programme } \\
\text { www.tekes.fi }\end{array}$ & Cluster oriented programme \\
\hline France & $\mathrm{FR}$ & $\begin{array}{l}\text { PREDIT } \\
\text { www.predit.prd.fr }\end{array}$ & $\begin{array}{l}\text { Network oriented programme } \\
\text { with thematic focus (transport) }\end{array}$ \\
\hline Hungary & $\mathrm{HU}$ & $\begin{array}{l}\text { KKK } \\
\text { www.om.hu }\end{array}$ & Competence Centre programme \\
\hline Ireland & IE & $\begin{array}{l}\text { Energy RDD Programme } \\
\underline{\text { www.sei.ie }}\end{array}$ & $\begin{array}{l}\text { Network oriented programme } \\
\text { with thematic focus (energy) }\end{array}$ \\
\hline Netherlands & $\mathrm{NL}$ & $\begin{array}{l}\text { TS } \\
\text { www.senter.nl }\end{array}$ & Cluster oriented programme \\
\hline Norway & NO & $\begin{array}{l}\text { TEFT } \\
\text { www.snd.no }\end{array}$ & $\begin{array}{l}\text { Network oriented programme } \\
\text { (with SME focus) }\end{array}$ \\
\hline Slovenia & SL & $\begin{array}{l}\text { Toolmakers Programme } \\
\text { www.uni-mb.si }\end{array}$ & $\begin{array}{l}\text { Cluster oriented programme } \\
\text { (with SME and thematic focus) }\end{array}$ \\
\hline Sweden & SE & $\begin{array}{l}\text { Competence Centre Programme } \\
\underline{w w w . v i n n o v a . s e}\end{array}$ & Competence Centre programme \\
\hline $\begin{array}{l}\text { United } \\
\text { Kingdom }\end{array}$ & UK & $\begin{array}{l}\text { Partners in Innovation } \\
\text { www.pii.org.uk }\end{array}$ & $\begin{array}{l}\text { Network oriented programme } \\
\text { with thematic focus (construction) }\end{array}$ \\
\hline
\end{tabular}


The graph below is an attempt to visualise the programme classification with regard to the following characteristics:

- science versus innovation driven programmes

- from short term project cooperation to long term networks and centre programmes

Exhibit 4: Typology of MAPs

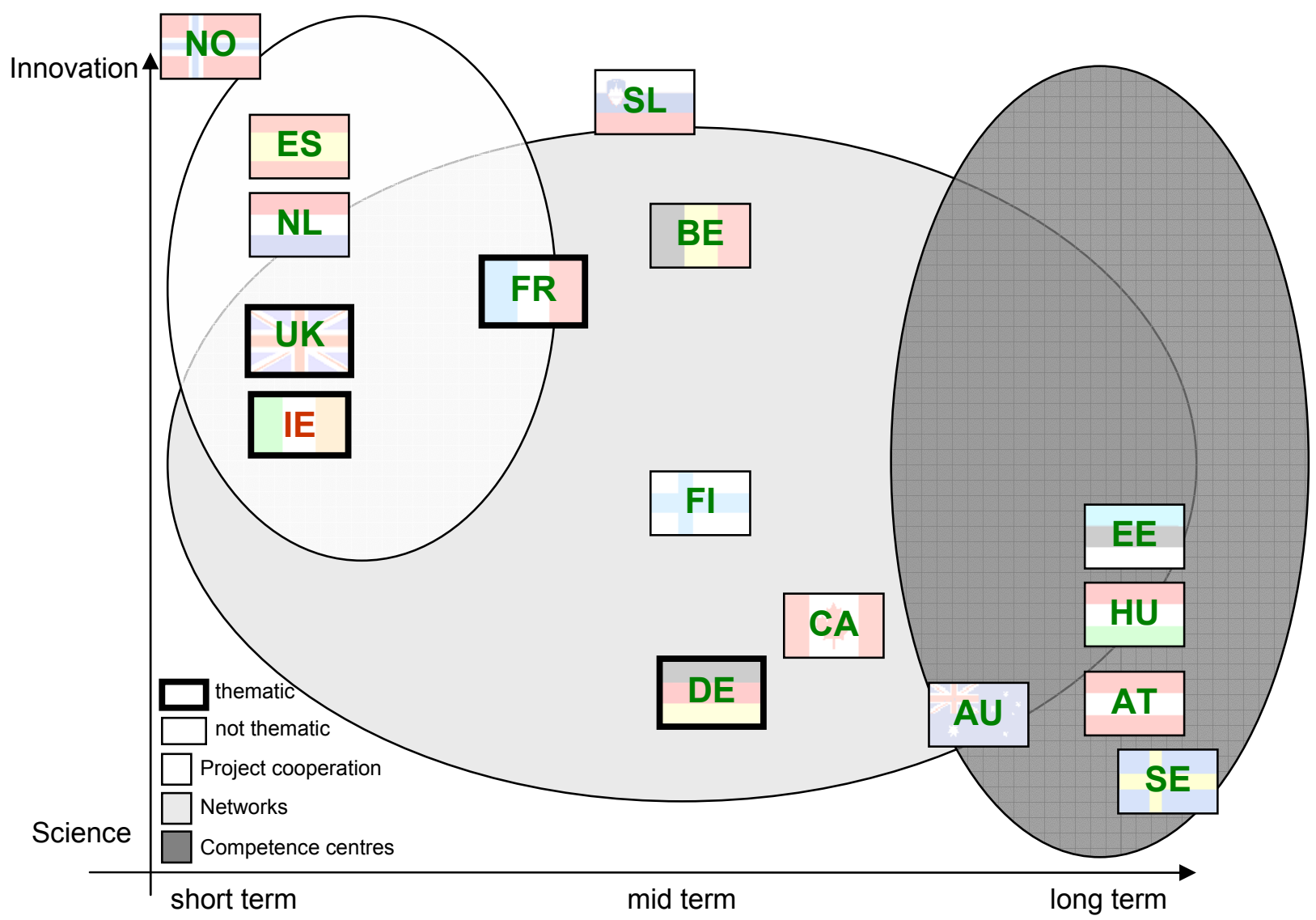

\section{$1.5 \quad$ Further remarks}

With the MAP-TN not only a stable platform for an open and frank exchange of experience based on confidence and trust was created, but it was also the starting point of further cooperation, staff exchange, and mutual collaboration as experts for evaluations, invitations as speaker to national workshops, etc...

From the beginning the MAP Thematic Network was supposed to be a learning platform for its members. E.g. this network was extremely valuable for EE as it ran parallel to the design process of the Estonian Competence Centre programme and a lot of the network's experience could be fed into the programme design. Also in Sweden a second call for Competence Centres was prepared at this time. 
MAP-TN can also be considered as a forerunner project of ERA-NET, covering at least stage one of ERA-NET, which is systematic exchange of information and good practice, including short-term exchanges of programme managers. Some MAP-TN partners consider going for the next ERA-NET steps. The ground for an even more in depth cooperation and further strategic activities was prepared, and also the questions of opening up of national programmes with regard to ERA were discussed.

MAP-TN wanted to go a step further, to disseminate what we have learned and let others participate in our exchange of experience: in the form of this handbook and the international conference.

Although these are more or less final activities of the network they could be considered as a starting point: a starting point for an experience exchange with you, the reader! We, the MAP Thematic Network, are very much interested in your experience, in your opinion, in your comments on the information provided, in the spirit of continuous organisational learning. 


\section{DEFINITION OF MAPs}

\subsection{Introduction}

In the MAP-TN we deal with RTDI funding programmes in the form of Multi Actor - Multi Measure initiatives with a focus on science - industry cooperation. Therefore a definition of MAPs had to be developed, based on a definition of RTDI programmes (see Annex) as a special tool of RTDI policy. So MAPs have to fulfil the criteria for programmes and a number of special criteria which are described below.

In our context RTDI funding programmes are a form of public support of RTDI activities. Such activities are performed by the private and / or the public sector. They are not identical with „RTDI programmes“, the latter meaning not the organised support, but the organised performance of RTDI activities, e.g. as an interlinked number of projects to achieve an overarching goal. The programmes provide the structural framework for the financial (or other ways) of public aid.

\subsection{Definition of MAPs}

In very general, MAPs are temporary answers to the complex settings and problems of innovation systems at regional, national and community level: We deal with funding programmes in the form of Multi Actors - Multi Measure initiatives ( $\rightarrow$ see Annex: definition of „RTDI funding programmes“). MAPs always have to fulfil the criteria for programmes and a number of special criteria, stated below.

Necessary elements constituting MAPs, discriminating them from other RTDI funding programmes

- Multi Measures: minimum two types of measures, in most cases more. Measures are activities within a MAP. In this context it is crucial to describe two levels of activities (i.e. measures taken) separately. Both levels should be in place, the second one must be there to constitute a MAP.

"Supporters" - Measures on the level of the programme: Nearly all MAPs have a whole set of instruments in their portfolio in order to intervene in complex settings within (a given part of) an innovation system. Such measures can be the delivery of e.g. project funding, other financial instruments, co-ordination and accompanying activities, information and / or other instruments. 
"Performers" - Measures on the level of the supported projects or initiatives: Here the focus is on the supported activities itself. The institution responsible for the MAP (i.e. generally the funding agency) uses one or a number of the instruments described above to support the activities defined as subject to funding in the set of goals and rules of the MAP. So financial support can be granted for research projects, demonstration projects, transfer activities, human resource development / exchange, networking, internationalisation activities, standard setting, spin-off activities and / or other measures within a consortium / a Centre / a group of cooperating actors. In this context it is important to note that the funding of different single activities without interconnections does not constitute a MAP.

For the further discussion we call "Multi Measures" only measures on the level of "performers" and to call measures on the level of "supporters" instruments.

Exhibit $5 \quad$ Typical examples for Competence Centre programmes and Cluster or Network oriented programmes

\begin{tabular}{l} 
Competence Centre programmes \\
\hline A funding agency provides as instruments mostly \\
financial, but also managerial support to consortia \\
according to a set of goals and rules. The consortium \\
consists of different actors (see below) who agree on a \\
structured multi-annual cooperation programme, thus \\
forming a Competence Centre. The centre and its \\
partners perform a number of activities like research, \\
technology transfer, human resource development etc. \\
in the form of interlinked projects and horizontal \\
activities (i.e. Multi Measures).
\end{tabular}

Network or Cluster oriented programmes

One or a number of agencies and other institutions support with a generally broad array of different financial and organisational tools the development of a whole interrelated sector in a particular spatial context. Generally rather loose cooperations between a large number of actors (see below, in different intensity) include RTDI projects but also managerial help, standards or export platforms, etc. (i.e. Multi Measures).

- Multi Actors: minimum two types of actors on the level of the "performers", i.e. the supported projects or initiatives. It is possible, but not enough if a number of "supporters", i.e. institutions / agencies together support single projects / individual actors without links on the level of "performers". Again, to constitute a MAP, the criterion of "Multi Actors" must be in place on the performers' level: Typical MAPs bring together different actors within innovation systems. Here science - industry cooperation is a typical feature of most MAPs. Multi Actors means therefore bringing together actors from different worlds, with different incentive systems, logic and cultures.

- System orientation: Nearly all RTDI funding programmes are problem-oriented; this means that they address specific questions and shortcomings that shall be solved. MAPs are always addressing problems, and those problems are on the level of the innovation system or a part of the system. A typical issue of MAPs is strengthening the links between different actors within such a system. Often system failures within a country / a region are the starting point for the design of a MAP. Note that this point is not fully MAP-specific, but all MAPs show such an orientation. 


\section{Further necessary but no discriminating features for MAPs}

- Programme character: See definition of RTDI programmes, (Annex); namely the characteristics "public support of RTDI activities", "existence of a structural framework", "set of goals and rules (regularly in the form of funding guidelines", "common umbrella" and "generally limited lifetime"). As MAPs are complex programmes, co-ordination issues are of special importance and in many cases an explicit, often external programme management is installed. As we talk about "RTDI funding programmes", mere planning documents or strategy papers are not MAPs. The term "RTDI funding programme" also excludes institutions, which can run / manage MAPs but are not MAPs or programmes themselves.

- Complexity: MAPs have a minimum level of complexity, resulting from the Multi Actor and the Multi Measure criteria described above. Therefore MAPs are always threatened by overcomplexity and role-conflicts. „Multi Goals“ is not necessarily an element of MAPs. Also note the strong interlinks between MAPs and the underlying institutional system. This point is not fully MAP-specific, but all MAPs show such an orientation.

- Evaluations: ex ante, monitoring and ex post with a particular focus on MAP-specifics and effects are at least stated in the set of goals and rules. (MAP-specific evaluation and monitoring features are covered in chapter 6).

\section{Optional features of MAPs occurring in most of the programmes}

- Competitive bidding for funds and public calls: Funding is in most cases given only after a competitive selection procedure. Quite often a mixture between top-down and bottom-up takes place. This means very often free choice of topics and formation of consortia.

- Two layers of "supporters": MAPs are in most cases managed / implemented by an external programme management or an agency commissioned by a ministry or a comparable institution. There are MAPs where one institution or funding agency has all the relevant tools in hand, while within other MAPs different institutions or funding agencies grant different forms of support with different tools. Due to varying policy settings, the supporting structures can be highly distinctive: Sometimes a ministry has a very strong role also in operative matters, sometimes specialised agencies have clearly defined sub-tasks in a MAP etc. Note that this point is not fully MAP-specific, but nearly all MAPs show such features.

- Specific learning elements and feedback loops: Due to the mentioned systems and Multi Actor approach most MAPs have some learning instruments.

So the most important features are: Multi-measures on the level of performers, cooperation between Multi Actors from different worlds on the level of performers and addressing of innovation system problems as the main driving rationale (see also chapter 9) 


\section{WHY ARE MAPs SO IMPORTANT?}

Since the mid 1990s, RTDI policy has increasingly utilised programmes which aim to bring about a structural change in national, regional and sometimes even cross-border innovation systems. These initiatives are often announced as structurally or thematically oriented competitions for the allocation of matching public and private funds: Consortia of applicants (public and private organisations) should formulate joint projects, goals and work plans in a selforganised form which formalise selection procedures. Partnerships of disparate institutions, like research institutes, universities, industrial companies, banks, technology centres, higher education establishments etc... aim, with a whole set of coordinated measures, at creating more effective local, regional or sectoral innovation systems. The promoted facilities receive funds to intensify research cooperation and networking. Such Multi Actor and / or Multi Measure programmes can extend either over regions and / or certain missions and / or selected technologies and branches. An overview of research and innovation policy measures in Europe from 2002 shows about 60 such programmes in the EU countries (www.trendchart.cordis.lu ). Two of the most prominent forms of MAPs are large science - industry cooperations like Competence Centres and rather network oriented and generally thematically focused cooperations like Cluster programmes.

To plan, promote, manage, run and evaluate such programmes is a considerable challenge for all actors involved, including ministries and funding agencies. So there must be good arguments, why they see an advantage in running MAPs, i.e. in embracing complexity. As we will see, the notion of innovation systems is of high importance in this respect - but is also important to see that MAPs are by far not the only instrument for public actors to intervene in systemic forms, there is also regulation, tax policy, higher education policy, other funding mechanisms etc.

\section{Some of the most prominent reasons are listed here in short:}

- As mentioned, innovation systems need tailored funding programmes. We will not repeat here the shift from the linear model and single actor orientated RTDI policy measures to the systems approach and it's emphasis on relationships and interconnections between actors (see Edquist 1997, Lundvall 1992 or Nelson 1993): This analytical and policy shift is well known and it is easy to see why MAPs are one eminent instrument of choice in this context. If the relations between different (types of) actors are crucial, RTDI policy had to develop appropriate funding mechanisms and tools.

- A related argument takes into account the specificity of regional and spatial cooperations. Both well-developed and less favoured regions see a considerable competitive advantage and development potential in defining strengths and potentials and in the subsequent coupling of different innovation-related actors in a region or a smaller nation-state (for examples see Bührer - Kuhlmann, et. 2003). Cluster programmes are a typical outcome of this strategy. Note that there are often also new actors like financing institutions, regional agencies or innovative service providers. 
- MAPs encourage interdisciplinary cooperations and are often formed to break up traditional boundaries between disciplines. This is more and more important for a number of industries facing complex innovation challenges which can only be mastered by inter- and transdisciplinary collaborations and networks - and skilled people like "industrial PhDs". Such networks also allow specialised SMEs to play a role and to profit from settings like Competence Centres.

- The argument of new cooperations and interdisciplinarity is also true within the research sector, namely the universities. MAPs help to overcome traditional trajectories and fragmentations in the light of the much discussed Mode II scientific production paradigm (Gibbons et. al. 1994). An additional advantage for universities to embrace many MAPs is their frequent design as mid-term and large science - industry cooperations. In times of tight budgeting, MAPs help to broaden the range of strategic funding partners.

- From a procedural point of view the arguments of behavioural change and learning matter most. Firms and research institutions have to adapt their routines, traditions and portfolios. MAPs on the one hand foster these change procedures, e.g. more management orientation in research or new forms of careers, within a well-funded mid term frame.

- Finally MAPs have the advantage to give a strategic push for whole fields without the need to build up everlasting institutions.

Of course not all arguments are of the same importance for all MAPs; but systems approach, behavioural change, new forms of cooperation, visibility of actors (and regions) always matter. In this respect we also believe that MAPs serve innovation systems better than single actor / single measure funding and financing instruments ... which nevertheless remain to be important for the functioning of an innovation system. 


\section{DESIGN OF MAPs}

\subsection{Introduction}

MAPs show - per definition - a number of particular patterns which influence the design process. They are usually implemented to address complex problems; they try to get whole fields moving in a certain direction, intend a behavioural and / or institutional change and are situated in a complex Multi Actor setting with the need to balance the different stakeholder interests, i.e. they follow a systems approach. Thus, as MAPs - often referred to as "innovation networks", "Cluster programmes", "Competence Centres" or "Competence Networks" programmes - are rather complex structures, a special situation arises with regard to the design of such programmes.

The design process of such a programme requires a number of clearly set steps on different levels:

(i) A profound analysis of the strengths and weaknesses of the own national (or regional) innovation system (NIS / RIS); (ii) the precise definition of what shall be achieved by the programme including appropriate and measurable aims and objectives; (iii) the analysis of the policy portfolio, i.e. other existing fun-ding programmes, policy initiatives and regulatory regimes as the danger of over-lap, confusion and "policy entrepreneurship" are high; (iv) the design of the tools of the particular MAP itself, $(v)$ the design of evaluation and monitoring procedures which take into account the particularities of MAPs; (vi) the question to what extent the different actors involved should be allowed to influence the programme design process; finally (vii) the question whether foreign participation shall be allowed and if yes, to what extent.

The design phase is of high importance and should be handled with care and transparency. If at this stage the roles, rules and playgrounds are not discussed and defined properly, unsolved problems will haunt policy makers, programme managers and funded consortia. It is especially difficult to repair a running programme or to define e.g. evaluation criteria from an ex post view. This being generally stated, we will not overload our report with hundreds of cross-references: The design process is prominent for the other steps in a MAP, described in the chapters handling "selection procedures", "contract negotiations" and "monitoring and evaluation". There is also a strong interlink between this chapter and the chapter on governance. MAPs strongly touch issues of regulation, actor sets, innovation culture and other governance-related issues. 


\subsection{Reasons for launching MAPs}

\subsubsection{Promoting innovation networks and other arguments for launching MAPs}

Modern economies can only thrive in the increasingly internationalised competition if they utilise their innovation potentials as widely as possible. In this connection, in the literature dealing with innovation science, it is argued that innovation processes pre-suppose the functional networking of different actors from industry, science, politics, regulation and education (OECD 1999, Caracostas / Muldur 1998). On which assumptions is this argument based? As long as research and innovation processes were understood as a linear sequence of complementary development steps practically and chronologically intermeshed and building on one another, from generic knowledge to the realisation of new products or production sequences, the prevailing idea was that the-se steps could be thoroughly organised and pre-planned. Meanwhile innovation processes are regarded mostly as recursive and interactive processes, in which there are not only different emergence contexts for innovation-relevant knowledge, but this knowledge is tested, if necessary modified, repeatedly invented and at the same time absorbed in other application contexts than those originally intended. So organisation and planning of RTDI policies get a different connotation.

The idea of networking different actors participating in the research and innovation process suggests itself in recent conceptions of complex innovation processes (Kline / Rosenberg 1986, Schmoch 1996, Rammert 1997, Burt 2003). Additionally a growing significance of research fields can be observed in which the results of basic research are of immediate interest for application and technological implementation and therefore a clear demarcation of research types is difficult (Mittelstrass 1994, Gibbons et. al. 1994). For such knowledge-based technology fields, science and innovation research could prove empirically that there is a closer interaction of application and basic research (Mode II approaches). Considerable inter-organisational cooperation between universities and the private-industrial sector $R \& D$ have been substantiated. The increase of interaction between industrial and university research (Schibany et. al. 1999, Nasin 1997, Meyer-Krahmer / Schmoch 1998, Schmoch 2003), especially since the eighties, is so large in the meantime that considerable repercussion on the research structures in industry and above all in universities can be assumed (Schmoch 2003). But: The interorganisational and cross-phase cooperation between institutions is in many cases still only moderately developed and must be greatly intensified, according to RTDI policy.

So innovation networks - and MAPs to stimulate them - are becoming increasingly important for innovation processes in modern economies. 
We assume that innovation networks arrange the required reciprocal linking of heterogeneous information and knowledge stocks as well as complementary resources better than hierarchic or market steering alone. Research and innovation networks are thus an institutional answer to heightened challenges in technology competition ${ }^{5}$.

Policy makers and innovation researchers have found also other reasons to come forward with Multi Actor - Multi Measure programmes. Such reasons are of course closely related to the above mentioned innovation system and Mode II approaches (Stampfer 2003a):

- Inclusion of new actors like innovative SMEs, the demand side (Arnold et. al. 1996) or public agencies from different sector policies like education, transport or environment into funding activities (Lundvall 2002);

- Securing interconnected long-term funding of project clusters without setting up new institutions;

- Integration of multi- and interdisciplinarity, as many MAPs address applied research needs for industry - and most of these challenges can only be met by approaches combining different disciplines and competences.

\subsubsection{Is it possible to create innovation networks ${ }^{6}$ politically?}

Innovation networks have a number of challenges for policy makers. They are of high importance for the functioning of innovation systems. Public actors can do a lot to ease birth and life of such networks: Awareness raising, partner search, building trust and a shared knowledge base, network organisation, provision of complementary resources and active cooperation can be typical public roles (Polt 2001). It would lead too far to analyse all these factors here but two questions seem to be central:

A first question in this respect regards equality versus dominance: If the principal economic policy regulatory question, whether the emergence of innovation networks should as a rule be left up to the self-organising powers of the innovation system, is answered with no, then the next question is, how should public promotional activities look like? A possible dilemma of public promoters can be that in Multi Actor arenas according to their self-conception they are the first among equals, in a network concept - and sometimes in reality - however only equal among equals (Kuhlmann 1998). As research on industrial districts, i.e. regionally anchored innovation networks shows (Crouch et. al. 2001), networks cannot be commanded, but are the product of a complex development process.

${ }^{5}$ Note that for us, Competence Centres are conceptualised as a form of an innovation network, though in some cases such Centres have to be set up forming a legal entity of their own. However, network characteristics such as non-hierarchical forms of communication, cooperation and decision within the competence centres are dominant.

${ }^{6}$ Note that also the creation of physical Cooperative Centres is to be understood as a measure to strengthen innovation networks of a multitude of different actors. 
We have to distinguish three issues here:

- Identifying structural weaknesses and/or system or market failures.

- Within networks there is generally no command and control, but of course dominant actors and less prominent players. We know this fact from running MAPs, often having one or few strong firms or professors as nodes, speakers, scyllas, charybdises etc. Also the social network analysis approach conceptually shows that there are different degrees of centrality and importance within networks (Barabasi 2002, Bührer 2002).

- The public actor(s) in such settings, namely the MAP managing and funding agency of course cannot dominate networks or centres funded by a MAP scheme. This fact does not mean that there is not a lot of power on the side of the agency, namely funding power and a strong role in negotiation processes. This strong role is mirrored on different levels: The public actors are defining the rules, funding guidelines and evaluation procedures, they select the peers and evaluators, they provide contract and report forms and impose additional requirements ${ }^{7}$. So there is still no command and control, but definitely no negotiations on totally equal terms!

A second question touches the issue of network creation by public policy. Generally it is wrong to welcome policy attempts to directly install or imitate innovation networks from the scratch. Public money alone is a bad incentive for scattered actors with no common work experience to team up. However, public policy appears promising in those cases where it can link up with traditional or latent innovation networks. Now such spatially located traditions cannot be found everywhere to link up to. At the same time there are innovation fields, in which networking takes place rather along the lines of certain subjects or technologies. This represents two special challenges to policy-making:

- Identifying excellent actors and actor combinations i.e. worthy to be supported and bringing them together and funding them overtime: Here appropriate - competitive - selection procedures, namely a good mix of bottom up and top down elements, gain especially attention - and MAPs are one chosen form of public action in this respect.

- "Network gardening": A second challenge for policy makers shows up in the forefront and around MAPs and similar funding programmes. While in MAPs the public agencies need a strong bargaining power, there should be a lot of preceding and accompanying "gardening work" of public and semi-public actors, not necessarily being funding agencies, as initiator, mediator and to some extent financial backer ${ }^{8}$. Public actors can do this kind of work because they should be trusted, having no particular interests.

${ }^{7}$ Compare the requirements for Competence Centres: instalment of boards, IPR rules, management structure etc. (see also chapter 6 on contract negotiations)

${ }^{8}$ Research on industrial districts (Silicon Valley: Saxenian 1994) shows that politics can be decidedly engaged, however not by means of harsh bureaucratic promotional measures, but by re-strained consulting. The state acts primarily in the role of a mediator (Kuhlmann 1998). In innovation research it is argued that it should be the role of policy-making in such situations to improve the resource situation in the network and support/ 
Finally it has to be said that design and management of MAPs are closely interlinked elements of one process, though often managed by different actors. Even some of the activities we called "network gardening" are part of a MAP preparation process. The more coherent a network oriented RTDI policy acts, the more harmonic different programmes - including MAPs - and other preparatory and accompanying measures will interact.

\subsubsection{The roles of MAPs in innovation systems}

MAPs are funding programmes and only part of an overall systems oriented RTDI policy approach. While "Multi Measures" and "Multi Actors" are always a necessary precondition within an explicit programme structure, the way of approaching different actor settings can be very different and distinct. (see chapter 2 on Definition of MAPs). Within our sample of MAPs and beyond we can roughly distinguish (i) network- / cluster-oriented MAPs and (ii) Centre programmes; the latter forming cooperatively funded and governed research entities generally called Competence Centres.

The function of all MAPs is to change actor settings and trajectories via temporarily funded projects or centres within programmes. On a policy level it is important to see that also other kinds of interventions like new institutions or regulatory measures could be possible answers to the challenges described above. The advantage of programmes including MAPs is their temporary, direct and adaptable character and their ability to influence institutional and systemic settings. Metaphorically speaking all forms of MAPs can be used in three following ways:

- As a kind of glue, linking together important parts of an innovation system, which would not link up at all / that fast / that strongly etc. A typical gluing activity is science - industry cooperation which is seen as crucial in innovation systems but faces a lot of barriers in both worlds. MAPs can contribute in this way to "produce" behavioural additionality - which in our case is also one of the most important evaluation criteria of such cooperation funding schemes.

- As a kind of solvent, changing rules, habits and incentives within subcultures. As we know from innovation research, trajectories always happen and they can be traps. Organisations find themselves locked in routines and need an incentive to change, to go into new kinds of research, new partnerships, new internal arrangements etc.

- As a kind of bypass, building roads around traditional blocks which are hard to change. Typical examples are strong departmental structures within universities or research institutions which will not perish overnight. MAPs can build ways around such disciplinary pillars by establishing new forms of long-term cooperations (compare ISI / KMFA 2004).

engender enthusiasm for new beginnings (Boekholt et. al. 1998). Bührer et al. (2002) recommend three corresponding policy instruments which are derived from the portfolio of all state technology and innovation policy: resource policies to activate the network potential, e.g. by financial promotional funds, infrastructure equipment, improvement of access to venture capital (VC); advice on strategic development potentials; "long promotional breath" corresponding to the long-term development tradition of grown models in order to achieve the necessary sustainability of network dynamics after the promotional measure is finished. 
To sum up: most MAPs are an answer to systemic failures in innovation systems. This means that a MAP is not primarily designed to help single actors to increase their individual competitiveness but to tackle problems relevant for whole (sub-)systems. The unique selling proposition (to the agencies' clients rather: the unique funding proposition) of MAPs is in our view that they have considerable systemic effects without building new organisations, without making new laws, without granting perennial benefits.

\subsection{4 "Multi-Problem": A MAP approach from the start}

Shortcomings in Science - Industry cooperation are one main reason to start such programmes and most of the MAPs under examination are strong in this respect. Of course there are also other forms of cooperations, namely vertical and horizontal inter-firm collaborations which are dominant in a number of Cluster programmes.

Our evidence shows different underlying rationales. Looking at the results of the MAP-TN and StarMAP, there can be problems on the academic side relating to a lack of incentives or subcritical masses (AT, DE, HU, EE, AU and CA) and / or on the industry side relating to a lack of absorptive power or short term planning horizons (AT, DE, ES, HU, SL, EE). Even if the subsystems are in a good shape there can be an urgent need to improve the communication and collaboration patterns between science and industry (SE, AU and CA). Some MAPs are designed to overcome structural weaknesses in sectors seen as strategically important for the future (DE, SL, IE, UK).

\section{Box 1}

\section{The Estonian CC programme - ivory and agony}

The launch of the Competence Centre Programme in Estonia was driven by the need to bring together the science sector and the enterprise sector to define possible joint projects in the form of a mid-term research plan. Strategic partnerships between these stakeholders shall be created within the innovation system. Earlier this kind of direct cooperation was not present, and the government sees the Competence Centres as the expected appropriate tool for the following problems: Low level of direct cooperation between science and industry; unexploited and potential market-oriented RTDI competence in both science and business sector; fragmented R\&D competence at the universities and/or their institutes; low level of RTDI capacity in the enterprises ("agony"); not enough skills in strategic planning and management in both science and enterprise sector (short-term and occasional funds-oriented projects at present, especially in the science sector); no entrepreneurial culture at the universities and their institutes ("ivory"); the older generation of the scientists is dominating at the universities and their institutes, not enough motivation for young scientists. 


\section{Box 2}

\section{The French PREDIT programme ${ }^{9}$ - moving to more network oriented transport research}

PREDIT was always motivated by a desire of multiple government bodies with an interest in land transport to increase coherence of their research activities. PREDIT 2 arose out of a decision to align the earlier and more traditional R\&D programme with the new model, the innovation network. The network model was seen to offer the possibility of expanding the reach of the programme especially within the private sector. Such an extension promised the possibility of more concerted and critical research investments as well as more credible and more effective diffusion mechanism as compared with a centralised programme. Since the launch of the first PREDIT programme, the technical focus has undergone some changes. PREDIT mainly financed the development of vehicle technology, while PREDIT 2 widened the scope considerably to include the organisation of transport services and the interfaces between those systems. Moreover, the focus on technology and engineering methods was broadened to include economic and social science approaches. PREDIT 3 is concerned with goods transport, environmental issues and security. Overall, the evolution in research priorities has involved successive programmes increasing their focus on demand-side and societal problems to do with transport rather than exclusively transport technology.

\subsubsection{Know your innovation system; Foresight and policy papers}

As MAPs try to give answers to systemic failures, it is necessary to analyse and understand the NIS. This includes a good data base, especially about the relevant actors and their needs, interests etc. Taking into account the logic of the different parts of the NIS and the different actors eases the assessment what (probably) works and what does not. For technologyoriented MAPs, foresight studies might be useful. However, the MAP-TN and StarMAP showed that just 3 out of 15 MAPs investigated took foresight activities in the design process into consideration (DE, IE, HU). Apart from foresight activities, own preparatory studies are good and necessary especially when MAPs are rather new to a given innovation system.

Policy papers played an important role in nearly all countries in the design process, be it an overall RTDI strategy (AT, EE, HU, FI, NL), an analysis about a given thematic field (DE, IE, SL) and / or an analysis of functional strengths and shortcomings in a given innovation system like the state of cooperation culture (AT, DE, $\mathrm{HU}, \mathrm{FI}, \mathrm{NL}$ ).

A further necessity regards having a clear picture on what shall be changed, respectively improved, in order to define the appropriate measures to reach these changes / improvements. It should also be seriously examined whether a MAP is actually a necessary and proper measure: As MAPs require a huge amount of preparatory as well as management and monitoring efforts, they are to be proposed and chosen with care.

\footnotetext{
${ }^{9}$ Programme de recherche et d'innovation dans les transports terrestres
} 


\subsection{Actors in the design process}

\subsubsection{No automatic actor synchronisation}

MAPs are per definition Multi Actor systems where different parties with different attitudes, interests, positions, strategies etc. come together in order to reach a joint set of goals. However, the different institutions involved in e.g. a Competence Centre or Competence Network like universities, non-university research institutes, large companies, small and medium sized enterprises do have different interests. These interests are subject to a series of negotiation procedures which have to be managed, mostly by the responsible funding agency.

The different actors have also - at least to some extent - considerable own means to finance their R\&D activities. The universities for example get basic funds (i.e. government, GUF) and project grants from foundations and research councils; large firms have considerable RTD project portfolios etc. Thus, the impact of a MAP on the behaviour of the actors is in fact limited. So MAPs are not all-powerful and not to be used as a panacea. So "No automatic actor synchronisation" means: Only the fact of starting a MAP and offering programme money is generally not a sufficient factor for involvement and behavioural change of public and private actors. They need to have a real incentive to take part in a MAP and the funding institutions have to have a clear picture about the incentives.

\subsubsection{MAP also means: Multi Actors in programme preparation}

Due to the complexity of MAPs and the Multi Actor setting it is principally useful to involve stakeholders in the design process in order to draw from different sources of decentralised knowledge, to stimulate the necessary dialogue between the different groups and to end up with a common understanding of what a MAP intends to achieve, the ways how to achieve the goals etc. Different groups mean representatives from all relevant actors like the different beneficiaries e.g. from science and industry as well as policy makers and agencies. As a MAP is not a law but a series of rules, negotiations and arrangements, communication requirements are quite high. Of course - we have mentioned it already - there is a certain power on the side of the ministry / the funding agency: They set the MAP agenda and they have the funding money. Nevertheless if they install a command and control system, their MAP will most probably fail. Additionally, in such a complex actor setting it is especially important to formulate clear roles of ministries, agency and stakeholders.

The involvement of actors already in the design process and in the process of the continuous development of a MAP assisted by, for example, a monitoring procedure, leads to community building, acceptance, commitment and learning, feedback and the formation of common expectations. However, as always there are also some potential disadvantages, especially the emergence or maintenance of a "culture of friends" and/or "culture of small commitments". One caveat: While in some countries like SE or FI a tradition of constructive stakeholder discourses seems to have evolved, other countries are full of "Bedenkenträger" (professional problem finders), and therefore the choice of stakeholders is a delicate process. 
In general all investigated MAPs include, as mentioned above, a variety of stakeholders in the design process, mostly in the form of expert groups/workshops. Some countries confronted these experts with detailed policy papers how the programme shall look like (AT, HU, SL), one country (IE) published the draft programme as basis for a written consultative process on the internet.

A general feature is that all design processes (initiated by ministries or agencies) carry out consultative processes with a number of different actors, like policy consultants or representatives from science and industry. An Advisory Research Council in form of a strategic governmental advisory board, which doesn't exist in all countries, was involved in the design just in two cases out of 16 (EE and CA).

A specific feature can be observed for so-called "thematic MAPs" as the involvement of additional actors very much depends on the subject covered by the respective MAP. E.g. UK's construction programme PII involved construction industry; Ireland's Energy RDD involved building developers, local authorities, energy agencies in the design process to ensure right from the beginning that results in these special sectors will be useful and taken up by the users.

Just AT, HU, EE reported about an efficient and fast design process where roles of the various stakeholders were clear which is considered as good practice. On the contrary the German MAP was developed through a very long and exhaustive design process, which included all relevant actors in the field.

\subsection{3 "And the dominant public actor is: ...."}

As the MAP-TN and StarMAP showed for the majority of MAPs investigated the ministries take the lead in the design process. An exception is SE where the former agency NUTEK (now VINNOVA) has set up the Competence Centre programme. This has to do with the Swedish system in general with their strong role for the agencies and their lean ministries. A similar example is FI with TEKES' Technology Programmes and the thematic MAP in IE, where the relevant agency Sustainable Energy Authority of Ireland, the former Irish Energy Centre, has had a very strong design role. A distinct approach was followed by AU (see details below) and CA: These countries started the programme design with a political announcement during elections. 
Box 3

The Australian CRC Programme ${ }^{10}-$ What the electorate usually swallows

The design process commenced in 1989 when an outline proposal of the CRC programme was presented by the Chief Scientist ${ }^{11}$, Ralph Slatyer, to the Australian Prime Minister, Bob Hawke. With the strong political backing of the Prime Minister a series of confidential discussions were held with other key Ministers, academics, research and business leaders in late 1989 and early $1990^{12}$. Yet before the formal approval by the Cabinet could take place elections were announced which resulted in the CRC programme becoming part of the election campaign "Clever Country". Had the Labour government not returned to power the programme might not have seen the light of the day as the other parties were opposing the scheme. When the next elections were held in 1993 the programme already had broad support and was not endangered any more. Immediately after formal approval of the programme by the Cabinet in 1990 the Prime Minister installed the CRC Committee and the CRC Secretariat in the Department of the Prime Minister and Cabinet. While the Secretariat developed the funding guidelines and prepared the first call for proposals the Committee was set up to provide recommendations to the Ministers and included representatives from the Prime Ministers Science Council, various government departments, stakeholders from industry, universities and $\mathrm{CSIRO}^{13}$ as well as the heads of other major government funding programmes. The Committee developed the selection criteria based on the underlying principles and objectives of the programme and set up two expert panels (physical sciences and life sciences) to play a key role in the selection procedure.

\subsection{Relations between overall and operative goals}

\subsubsection{Goal formulation: a minefield with time fuses}

The definition of appropriate goals - and the design of evaluation procedures able to measure the level of goal attainment - is a crucial factor in the design process. Of course all RTDI funding programmes, not only MAPs, have to have a number of written goals. But there are some MAP specific challenges which should be taken into account.

- Due to the experimental character of MAPs they necessarily show broad overall goals, but there is an absolute need for operative goals. By being larger and more complex, MAPs also create higher expectations on the policy level - this might be a danger especially with regard to goal overload.

${ }^{10}$ Cooperative Research Centres

11 The Chief Scientist is appointed by the Australian government and serves as an advisor in all science and technology related policy issues.

12 Slayer (2000)

${ }^{13}$ The Commonwealth Scientific and Industrial Research Organisation (CSIRO) is the largest government owned research organisation in Australia. Website: www.csiro.au 
- There is always a goal conflict between structural goals and the limited time horizon of funding: Multi Actor systems are created; they develop their own lives and a deep interest to ensure a long-term existence which is a MAP goal. MAP funding on the other hand stops after some years and it is often not possible or wise to plan self-sustaining initiatives: A common MAP specific goal is to create self sustaining networks but this is often a naive idea: ambitious networks / structures / organisations will not survive without some public money and without a kind of "MAP governance", namely if real scientific research shall be performed (see chapter 8 on governance).

- As behavioural and institutional change is important for MAPs, quantitative goals are only to be applied in a limited form, namely impact on firms and some output indicators. Hence, qualitative goals are always necessary elements of MAPs (see chapter 7 on evaluation and monitoring).

- As most MAPs show a large range of different aims, conflicting goals may occur, for example: the maintenance of scientific excellence, thus following the rules of basic science with a large amount of freedom, and an accelerated time to market; or as an other example: long term research and SME inclusion. Such conflicting goals should and can be dealt with in advance, often being easy to solve at this stage: With proper rules science can flourish and be relevant.

\subsubsection{Formulation of goals and evaluation design as one interlinked process}

A crucial point with regard to monitoring and evaluation procedures is the synchronisation of goal formulation and evaluation procedures before starting the MAP, e.g. in the design phase it is necessary to formulate goals that are suited for evaluation. Generally spoken, evaluations are more difficult than in other programmes (see chapter 7 on evaluation and monitoring): As mentioned above, MAPs pursue a lot of aims, quantitative and qualitative, many of them with a structural character which means that they are very difficult to measure - at least in the short run. In the design process attention should also be given to the definition of relevant indicators. As MAPs are complex, goals and criteria and also indicators are multi-dimensional. However, summative performance measurement rapidly reaches its limits. For this reason formative, learning-oriented evaluation approaches can and should be used - partly in competition, partly complementary to the summative ones. On the basis of multiple evaluation results on the relationship between programme targets, measures and impacts, spectra of alternative paths of further development of a programme can be shown, alternatively regarding adjusted targets and differing starting points, addressees and time and objective range. Such information can aid the actors to play their strategic games more transparently and "more effectively" in the general interest. 


\subsubsection{Scoring goals ... and scores of goals: Some empirical findings}

We can always find some "strategic" goals showing the main direction of the programme. Most of the MAPs included in the MAP-TN and in StarMAP have strong systemic goals, aiming at a better functioning of a given innovation system (AT, DE, SE, HU, SL, FI, FR, NL, AU and CA), while other more thematically oriented MAPs focus on the overall goal of strengthening industry's competitiveness (UK). Operational goals can be found in all programmes, but in different forms. Some of the MAPs have one overarching goal (AT, UK, SE, FI, FR, NL, AU and CA), others have a number of strategic goals, each being operationalised (see example ES).

Note that a number of MAPs do not feature quantitative goals (AT, UK, SE) though there is a strong link to evaluation procedures in a number of cases. This fact has apparently not so much to do with a lack of evaluation culture, as Scandinavian or Anglo-Saxon countries do have a long-standing evaluation tradition, but with obvious difficulties to formulate quantitative output or impact goals for MAPs, especially Competence Centres. As MAPs regularly address a number of actors with a number of instruments, clear predictions about individual measurable outputs and impacts are more difficult than in any single-issue programme. Nevertheless there are MAPs with measurable quantitative goals (DE, ES, SL, NO), some even show a disproportion between instruments available and a quite sizeable number of goals (DE). The link between goal setting and evaluation procedures is there from the beginning in all MAPs. A number of them show rather detailed work from the beginning (DE, HU, EE, SL, FI, NL, AU and CA), though there are other examples where quantification and detailed operationalised evaluation settings had been prepared some months after the MAP started (AT).

\section{Box 4}

\section{The Basque PCTI Cooperación programme - We dare to quantify}

Overall goals (also socio-economic): Fortification of the agents of the RTD system; The generation of new knowledge as a high priority area; Integration of the RTD system; Promotion of the technological innovation within SMEs; Correct use of the advantages of the Information Society Technologies; Generation of new products and companies as a result of the new knowledge.

Goals on the macro level: To increase the percentage of the Basque Gross Domestic Product spent on Research and Development (1.2\% - 1,7\% in 2004) and the companies expenditure in RTD (65-35); To equal the number of researchers to the EU average (5 researchers per 1000 active people).

Operational goals: To improve the competitiveness of the companies of the Basque Country, supporting research activities, technological development and innovation, and collaboration between the technological users and suppliers.

Quantitative goals: Participation of 300-400 SMEs in the different areas of the programme; Generation of 200 new commercial products.

Qualitative goals: Identification of the high-priority areas of knowledge for their development; Regional infrastructure of the knowledge; Commercial use of the RTD results; Opportunities generated by the Information Society Technologies; Promotion and development of international relations; Improvements in the management of the SMEs (standards of quality). 


\section{Box 5}

\section{The Dutch TS Programme ${ }^{14}$ - Impact assessed}

The aim of the TS is to increase the R\&D investments of Dutch firms and the return on these investments. This objective is defined in a qualitative manner; there are no targets for the increases in R\&D investment or the return on these investments. The programme's objective is "operationalised" by: technological success of projects, cooperation process and economic perspective of projects. In order to assess the achievements of the programme, EZ (Ministry for Economic Affairs) and the agency Senter set up a system for monitoring and impact assessment in $2001^{15}$.

The programme's achievements will be monitored through a set of indicators - based on the three criteria above - that are measured at the beginning and the end of the programme. With respect to the impact assessment, this will be done annually, asking project leaders who have completed their project about the project, the cooperation, the project's outcome and its continuation ${ }^{16}$. The development of the monitoring indicators was not yet included in the design of TS; it took place after the start of the programme. Therefore, there has not been a measurement at $t=0$. As a result of another streamlining of EZ policy instruments, a new cooperation scheme will be developed in 2004. The development of indicators for monitoring purposes will be included in the design of this new measure so as to permit a measurement of the target variables at the start of the programme $(t=0)$. This allows for a more critical analysis and checking at the end of the programme whether the objectives are achieved. With respect to ex post evaluations it was only specified in the design process that evaluations take place once every 4 years and that they are carried out by external evaluators.

\subsection{MAP guidelines, underlying documents}

\subsubsection{A need for own funding guidelines?}

MAPs are situated in complex actor settings having to balance the different actors' interests. Furthermore, MAPs are rather new policy approaches being characterised by a high amount of uncertainty and risk to fail, the social setting of consortia or networks adding risks to the "normal" technical risks. The risks have to do with the Multi Actors setting as communication becomes more difficult due to different expectations, values and "languages". Furthermore multiple goals might imply also conflicting goals and criteria which have to be addressed. The risk is high not to reach all formulated goals.

14 Technologische samenwerking

${ }^{15}$ Along with the streamlining of its firm-oriented technology policy instruments, EZ also expanded monitoring and impact assessment of the instruments. More specifically, the Ministry laid down the following principles: (i) to adopt a systemic perspective in assessing the effectiveness of innovation policy and (ii) to develop a strategy for data collection at the start of every policy action. This last action includes setting up a monitoring system with a number of specific indicators and linking of different data bases. (iii) to give evaluation a more central position within the organisation

${ }^{16}$ Dutch Ministry of Economic Affairs / Senter (2002) 
The larger size of the programmes and subsequently their higher visibility contains risks in particular for the implementing agency as the possible non-fulfilment of expectations is followed more closely by the policy level. Consequently, clear and transparent rules become essential.

For policy makers the question now is whether traditional and well-tried programme rules and guidelines $^{17}$ are sufficient or whether some specific MAP guidelines and rules are needed. There are several arguments favouring MAP specific guide-lines, i.e. formal guidelines and information documents:

- As MAPs are rather new policy instruments situated between different sub-systems, e.g. science and industry, standard solutions or tools from those different sub-systems might fit only partly.

- Additionally MAPs are based on and need intensive communication and negotiation patterns: clear and transparent rules which are directly related to the specific MAP challenges can ease the communication within the centres / networks / clusters and their environment and thus avoid misunderstandings and frustration.

- Clear and transparent MAP specific rules can also help to avoid the dominance by informal rules: informal rules often favour old boys networks and existing structures, things which are in most cases intended to be changed by a MAP. It is always difficult to decide how far policy makers shall trust the beneficiaries: mistrust can refer to two reasons: deceiving or misunderstanding. Potential for the latter is higher in MAPs due to their complexity and due to their bridging function. This is especially true in situations, which are characterised by a high level of uncertainty.

- A further argument promoting specific guidelines is the risk to fail (also for the MAP agencies) in view of the large amount of public money usually linked to MAPs: clear rules especially with regard to the selection procedures might help to avoid failure: even if the competitive bidding argument is rather size-specific than MAP-specific, MAPs are especially endangered to chaos and micro-politics. New intentions, approaches and logics cannot rely on "he was always a good guy"-mechanisms.

In general, there is a certain trade off between rules (be they specifically designed and tailored or be they the overall programmes rules) and the freedom to achieve goals by steering more freely: every programme actually needs a certain set of clear and transparent rules, but it is naive to think that there is no scope of interpretation: again, as always in the MAP context, communication abilities are becoming important in order to make these unavoidable interpretations or deviations from the rules transparent and accepted. Additionally, as rules are defined in advance of the start of the programme, one should install certain mechanisms to adopt them according to new insights and needs, for example following the results of monitoring and evaluation procedures.

17 Guidelines in a context are rather hard, normative sets of rules for programmes rather than soft explanatory papers 
The advantages and risks of specific MAP guidelines and underlying documents are summarised in exhibit 6 :

\section{Exhibit 6: $\quad$ Specific MAP guidelines, underlying documents}

\begin{tabular}{|c|c|}
\hline Advantages & Risks / Possible Disadvantages \\
\hline $\begin{array}{l}\text { Transparency and clear information; funding guidelines for } \\
\text { all actors (policy level, managing level, project level). }\end{array}$ & $\begin{array}{l}\text { Less opportunity to develop standard tools for all national } \\
\text { RTDI programmes. }\end{array}$ \\
\hline $\begin{array}{l}\text { Greater fairness - requirements are clear for the } \\
\text { proposers. Insider information is not (so) important. }\end{array}$ & $\begin{array}{l}\text { Higher information costs, experience and expectation of the } \\
\text { target group. Learning effects are contradicted if every } \\
\text { (new) programme has own rules. }\end{array}$ \\
\hline $\begin{array}{l}\text { Specialised knowledge is created in the developing and } \\
\text { implementing agencies (knowledge where to get relevant } \\
\text { information, what structures are appropriate). }\end{array}$ & $\begin{array}{l}\text { Specialised knowledge within the agency may be a pitfall } \\
\text { because it lays to dependencies and lock in - effects. }\end{array}$ \\
\hline $\begin{array}{l}\text { Potentially lower costs of design, implementation, } \\
\text { enforcement and monitoring: Better to have clear, MAP- } \\
\text { specific rules than a patchwork of ill-fitted traditional rules } \\
\text { and guidelines }{ }^{18} \text {. }\end{array}$ & $\begin{array}{l}\text { Potentially higher costs of design, implementation, } \\
\text { enforcement and monitoring, if variety of public rules / } \\
\text { guidelines becomes too high }\end{array}$ \\
\hline
\end{tabular}

\subsection{2 "It's the law!" Is it? - Some empirical findings}

There are two issues worth looking at: Firstly is there an overall funding law with strong impacts and rules how to draft MAP rules and guidelines? Secondly are there specific written MAP guidelines?

- As MAP-TN and StarMAP shows, many countries do not have a general RTDI funding / organisation law determining programme design, management and evaluation. The general legal basis for designing programmes is in most cases to be found in general funding guidelines or general provisions for public funding (AT, DE) or the statute of the agency in charge $\left(S E^{19}\right)$.

- Some programmes are based on special ministerial acts (below "law" level) regulating the individual MAP (AT, ES ${ }^{20}$; guidelines as a formal result of the design process); while others are designed within the responsible ministry or agency based on a good practice approach (SE, UK, HU, AU).

${ }^{18}$ Critical examples are some recent Austrian thematic programmes with strong MAP characteristics. Their set of rules can be seen as a patchwork of traditional "single innovation project funding rules", rules stemming from the long standing ministerial project funding (“Auftragsforschung”) and new elements.

19 VINNOVAs RTDI-support is based on the act $(1995: 1254)$ about state aid to technical research, industrial development and inventions. This act is decided by the government and notified and approved by the Commission. In summary: In a broad sense these guidelines have no legal char-acter but the act mentioned above allows us to allocate the support to the CC 
In MAPs (like in other programmes) we do not only talk about hard and formal rules like funding guidelines, contracts or reporting procedures, but also about softer factors like traditions or good management procedures (see also chapter 8 on governance). Rules are also strongly determined by the way of support, i.e. by the "Multi Measures"; compare in some Competence Centre programmes the availability of large sums of RTDI public funding money (AT, HU, SE, $A U$ and $C A$ ) versus the reliance on cooperation costs only in other programmes (DE).

Another rule-related issue is the level of freedom for consortia to develop their plans and organisational cornerstones. Most MAP managers in the MAP-TN consider their programmes as rather liberal: MAPs provide a number of legal, organisational and financial frameworks (e.g. legal structure of a consortium, project orientation, accountability requirements, organised and well documented planning procedures ...), within these frameworks the actors supported are free to plan and to design and to run their research programmes.

Finally, in most programmes the formal funding decisions are a ministerial prerogative (AT, DE, $\mathrm{HU}, \mathrm{SL}, \mathrm{FR}, \mathrm{AU}$ and CA) but there are exceptions; like SE, where VINNOVA is taking the formal decisions.

\section{Box 6}

\section{The Austrian K plus programme - prominent policy papers, strict guidelines}

There is no "Competence Centre law" or comparable document issued by parliament to establish the programme. Two 1996 / 1997 government policy papers (BMWVK 1996, Hochleitner / Schmidt 1997) stipulated the start of a CC programme including some first conceptual frameworks. The design process of the programme started in the end of 1997 with a 50 page policy paper, the so-called $\mathrm{K}$ plus "Vorhabensbericht". All the cornerstones of the planned programme were issued and made subject to public discussion. On the basis of the policy paper the funding guidelines ("Richtlinien für die Errichtung und Finanzierung von Kompetenzzentren "K plus", 1998, rev. 2001)" were written. The legal basis for these guidelines are the general provisions for public funding by the Ministry of Finance („Allgemeine Rahmenrichtlinien für die Förderung, BMF 1977). As a next step the programme was notified in the EU DG Competition procedure for national RTD state aid. After that a number of manuals for reviewers, proposers, evaluators etc. were written and made public. Note that contrary to such programmes other (project oriented) funding initiatives and organisations, namely the two big RTD funding organisations FFF and FWF have been established in 1967 based on the Austrian Research Funding Act. 


\section{Box 7}

\section{The Finnish Technology Programmes - let's remain flexible}

The funding in Technology programmes follows the general principles and criteria of RTDI funding defined by EU commission R\&D framework, Government acts concerning grants and loans and funding principles and criteria defined by Tekes based on the previously mentioned framework and acts. Each programme can and usually does define additional criteria to focus and direct RTDI within the programme to a specific direction. These additional criteria are defined in consultation with key stakeholders. Furthermore, there are guidelines for programmes for defining IPR rules and contracts. Practices related to selection procedures, monitoring practices, etc. are mostly tacit knowledge, not documented in detail. This has been partly intentional to allow for a sufficient degree of innovativeness. Most of these are currently documented for each programme separately to allow for transparency and exchange of good practice, but still the level of general programme guidelines is intentionally left to the level of guidance and check listing, not taken to the level of detailed instructions, rules or procedures. There is a manual of about 15 pages for tasks of the programme manager and the steering group. The rules are flexible, just giving an orientation and supporting the programme steering. The necessity to adapt the procedures and programme structure to the specific objectives and the structure of the corresponding sector is emphasised. Additionally, there exists a general guideline for evaluations of technology programmes.

\subsection{International influence and opening up of national programmes}

\subsubsection{International influences and pedigrees}

In the evolving European Research Area (ERA, European Commission 2000), international influence and interplay becomes more and more important and takes place at different levels, for example more international research alliances and networks, the opening up of national programmes, the involvement of foreign peers in selection procedures etc. However, it is essential to take the importance of the framework conditions into account: As Good and even Best Practice approaches grew highly popular namely in the OECD (OECD 1998a, 1998b, OECD 1999b) and EU context and it became fashionable to import foreign programme concepts, there is a certain danger to underestimate different legal, organisational, cultural frameworks and questions like budgets, size, past success etc. (see also chapter 8 on governance). Nevertheless especially MAPs need careful comparisons on management level due to their complexity and their specific problems as for example the lack of a dominant actor and the careful balancing of the different actor interests.

A further entrance door for international influence in the MAP context is the strong involvement of foreign peers in ex ante, monitoring and ex post evaluations which is caused not only by scientific necessities or country size but also simply by the fact that MAPs are very often large programmes addressing the most relevant national actors so that competent peers have to be looked for abroad. A further advantage of foreign peers is that they can feed in their experience, often stemming from their own MAPs. 
Finally, foreign peers ensure - at least in most cases - independence and thus fairness. Arguments against the strong involvement of foreign peers are that they are not familiar with the typical challenges of the individual NIS, that they are not strong enough in their judgements because they do not compete for the same money as national peers would do, and the difficulties, efforts and costs to identify and motivate foreign peers to participate in the national evaluation procedures. Mixed peer groups are one possibility to avoid most of the shortcomings.

As the results of the MAP-TN and StarMAP show, most MAP design processes are characterised by a strong "international good practice" approach. Policy planners and experts study foreign examples, go abroad and try to import apparently well-working MAPs (Stampfer 2003b). In addition, international experts are often involved in ex ante evaluations, programme design and related studies.

Box 8

\section{The German Nanotechnology CC Programme: A programme under the Influence}

The BMBF commissioned Technopolis to investigate the international initiatives to build, develop and support „Kompetenzzentren“ (Boekholt et al. 1988). The following initiatives have been studied in the report: Silicon Valley and Boston 128 as classic cases of entrepreneurial CC, Cambridge (information technology) and California South Bay (Laser Technology) as science and technology-led CC, Eindhoven and Gothenburg as Industry-led CC and Scotland (Silicon Glen), Japan (Kanagawa) and Hsinchu Science Park as Policy-led CC. The studied examples showed that the emergence of well-functioning $\mathrm{CC}$ needs a long-time horizon and can hardly be influenced by policy makers. However, the particularity of the German CCN is that no R\&D grants are directly involved which is really unique.

We can see also families and "pedigrees" of MAPs, which is illustrated below:

Exhibit 7: International Influence on the Design Process

NSF Engineering Research Centres, ERC,

USA 1985

CRC Cooperative Research Centres,

Australia 1990

Competence Centres,

Sweden 1995

K plus Competence Centres,

Austria 1998

KKK Competence Centres,

Hungary 2000

Competence Centres,

Estonia 2003

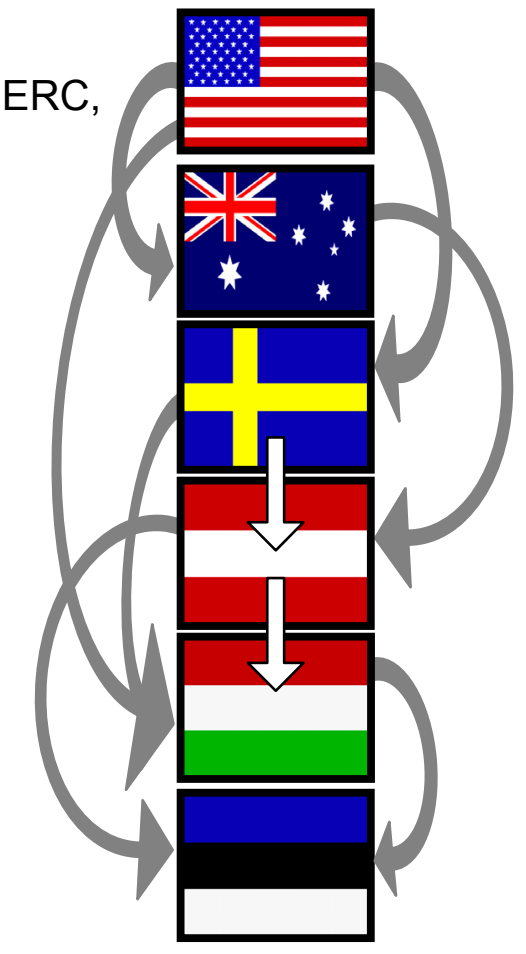


The making of typical Competence Centre funding programmes is a story of its own: The mother of all of them is the Engineering Research Centres programme (ERC) issued by U.S. National Science Foundation (NSF) in the Eighties. It directly influenced at least the Australian Co-operative Research Centre (CRC) programme and the Swedish CC programme (SE). Austria studied for the $\mathrm{K}$ plus programme the Australian, the Swedish and a Canadian example (AT), while the Hungarian KKK programme is based on the U.S. model primarily, taking into account Swedish and Australian experiences in the design and the Austrian experiences for the running of the centres. The same holds true for Estonia who studied the Hungarian and Swedish examples and got hands-on input from the Austrian programme. The European Framework Programmes played an important model role in at least one case (IE).

Some of the programmes ( $C A$ and $A U$ ) studied within the StarMAP project were quite unique when they were set up around 1990. These countries looked at foreign studies but could and did not carry out "fact finding missions".

\subsubsection{Opening up of national programmes}

Within the evolving European Research Area and with the increasing globalisation of industrial RTDI performers the national/regional RTDI funding programmes face a challenge to open up their programmes to a certain extent to foreign participation (see also Stampfer 2003b). Additionally, specific goals in some MAPs are to create institutions or networks with the aim that they should perform better on an international level than the individual actors could. The opening up of national programmes thus becomes a crucial issue also for MAPs: foreign actors can participate in a funded project. The aim of policy makers is that foreign project participants might contribute to the international competitiveness of a country. The aim of a foreign participant in a project is to create an individual win-win situation.

However, most of the MAPs under review take a rather cautious approach. This means that foreign participation is possible but not actively promoted (UK, DE, ES). Competence Centres are relatively easy to handle as the centre itself has its location in the "home country" of the RTDI funding programme (AT, SE, HU, AU and CA). Here and in other programmes often the question of reciprocity arises (SE). Some MAPs treat foreign partners and partners from the home country equally (AT, SE, HU, AU) while others provide open access but no financial support (DE). Most MAPs (also other RTDI funding programmes) changed their relevant evaluation criterion from "Do participants have research/production facilities in my country?" to "Is the project proposed really useful for my country?" (IE, HU, AT).

The StarMAP examples AU and CA introduced a specific clause: In Australia it's called the "capture the benefits of research for Australia" clause, as in the past there were obviously a number of incidents (commercialisation of know-how outside Australia without significant returns, purchasing and closing down of Australian companies by foreign "mother" firms. However "international collaboration" is part of the selection criteria for projects. Canada has also set up a similar clause "Benefit for Canada" for the same reasons. 


\section{Box 9}

\section{The Austrian K plus programme - Internationally minded centres ${ }^{21}$}

Foreign firms can take fully part in the funded Centre Programme: Max. $25 \%$ of industrial share (i.e. of the minimum $40 \%$ private contributions) can come from Non Austria-based firms. The issue is the location of a firm, while the nationality of the owner does not matter at all. The inclusion of foreign firms is checked via some ex ante evaluation sub-criteria: Usefulness for centre (plus strategic perspective: e.g. multi firm projects, specialised knowledge) and Usefulness for Austria. K plus funding and also all firm contributions go to the centre, there is no direct funding of firms in the $\mathrm{K}$ plus programme. Foreign industrial partners have the same rights as "Austrian" firms. About $10 \%$ of the programmes' industrial partners are foreign. Foreign scientific partners can be core partners or loosely cooperating partners: This depends on the coherence of scientific proposers, special knowledge, etc. If they contribute to the centre they are treated like Austrian scientific partners. PhDs, exchange of personnel, etc. Incentives for cross-border cooperation: Evaluation criteria (based on goals) as incentive, one of these addressing "Participation in international RTD programmes".

\section{Box 10}

\section{The Finnish Technology Programmes - Internationalisation as core aim}

On of the aims of technology programmes is to support the internationalisation of R\&D in Finland. This takes place on two levels: Tekes, the national R\&D-funding agency, actively encourages cooperation on programme level and is eager to be involved in the preparation of joint technology programmes in cooperation with other funding authorities in different countries. Such programmes should focus on international cooperation and how this can provide added value to the participants. Funding authorities agree on the general principles of funding and the interpretation of the results (IPR). Typically, international cooperation is accomplished in the field of basic research. Additionally, enterprises are encouraged to collaborate with their foreign counterparts as technology programmes provide a good framework for collaboration. Foreign research institutes, universities and enterprises benefit from direct access to research and development projects. The programme management organises opportunities for building partnerships between foreign companies and programme participants. Relevant costs of participation are primarily covered by the foreign entity itself or by national funding from its own country of origin.

\footnotetext{
${ }^{21}$ OECD (2004) $22 \mathrm{f}$
} 


\section{The Canadian NCE Programme: Benefits for Canada}

The Networks of Centres of Excellence programme (NCE) encourages international participation, but does not allow networks to supply NCE funding to researchers outside Canada. Current granting council rules do allow foreign researchers to obtain up to three months' travel expenses when visiting Canada in joint research projects. Also Networks can use partner funds to support research abroad. The basic idea behind that is the "Benefits for Canada"-clause. In the past there were obviously a number of incidents (commercialisation of know-how outside Canada without significant returns, purchasing and closing down of Canadian companies by foreign firms) that put the idea of "Benefits for Canada" high on the political agenda. This is also reflected in the evaluation criteria: "The extent to which the pro-gram will contribute to Canada's ability of lead in areas of research with high economic and social impact." In recent years there has been a growing emphasis on attracting international companies to do research in Canada, e.g. via a very generous tax system, while at the same time trying to protect Canadian IP.

\subsubsection{International regulations, competition law}

In a MAP design context it is also important to see the potential impact of regulatory issues, coming from other policy fields than RTDI policy. A prominent example in the European Union is competition policy with its aim to prevent market distortions by public subsidies. Though there is a positive approach in European competition policy towards research and innovation, there is nevertheless the need to regulate funding programmes with a potential to distort competition in the Internal Market. There are also maximum funding quota and other regulations. If MAPs have a strong and direct impact on industry RTDI budgets (i.e. a direct funding of industry is the result of the MAP), a notification procedure at DG Competition in Brussels - and a subsequent reporting requirement - can be part of a MAP design process. Some European MAPs do not include direct support to industry, others do. For the preparation process of all MAPs in the Union there should be a procedural step, where this issue of possible notification has to be cleared by the programme planners. Non European countries (CA, AU) of course are not subject to this regulation and can sometimes act more freely. (For the impact of regulations from other policy fields see also chapter 8 on governance). 


\subsection{Lessons from the MAP-TN and StarMAP}

Based on the experience of the MAP-TN and StarMAP we can formulate the following recommendations for the design process:

1. Know and analyse your (own) innovation system because of the nature of MAPs giving answers to systemic failures. In this context it is useful to have a good data base, including policy papers and international comparisons, especially in those cases where the MAP is new to the country. Foresight studies may help, but have to be designed and analysed carefully.

2. Know your regulatory, cultural and institutional environment: It is essential to take the importance of the framework conditions into account. As Good and Best Practice approaches grew highly popular namely in the OECD and EU context and it became fashionable to import foreign programme concepts, there is a certain danger to underestimate different legal, organisational, cultural frameworks and questions like budgets, size, past success etc.

3. Conclude whether a MAP is a necessary and proper measure. If it is, come forward with strong pictures and good public relations. Make yourself understandable, a MAP is not always clear and appealing to the public, to a firm or to a scientist.

4. Come in with a rather strong picture on what shall be changed, improved, achieved and / or strengthened and how and why. Otherwise policy makers and programme managers might be misguided and confused by the high complexity of MAPs. This can make the programme vulnerable to lobbyism, i.e. the domination of stakeholders' interests. Create a basic, innovative idea on how to bring about the improvements.

5. Make clear and verifiable goals, which does not always mean quantifiable goals: The definition of appropriate goals - and the design of evaluation procedures able to measure the level of goal attainment - is a crucial factor in the design process: Programme designers have to take into account that most MAPs show broad overall, structural goals which are difficult to measure and that they are endangered by goal overload.

6. Take time to prepare the programme and bring in stakeholders. Due to the complexity of MAPs and the Multi Actor-setting it is principally useful to involve stakeholders in order to stimulate the necessary dialogue between the different groups, to draw from their different and decentralised knowledge and to end up with a common understanding of what a MAP intends to achieve, the ways how to achieve the goals etc. Consultation processes at the beginning are essential for informing and building commitment which has to follow clear rules and a clear division of roles. In an ideal case there is an interactive MAP preparation process and other related measures of consensus building, like discussion platforms, RTD advisory councils or seed money for networks. 
7. Transform the basic idea (see recommendation 4.) into some simple meaningful pictures and slogans to transport the most important messages. Communicate these pictures actively, do it everywhere and do not stop this task. It really takes time to communicate MAPs and to anchor the message.

8. Take into account the logic of the different parts of the national innovation system and the different actors and study closely what works and what does not; take into account the logic of the different organisational players in the arena.

9. Set clear and transparent rules which are directly related to the specific MAP challenges like the different actor logics and interests. Stick to them and be predictable. Think twice before you change rules (also because you have to communicate them, see 7 .).

10. Authorise a competent funding agency which is close to the performing actors but independent enough to avoid domination by particular interests. Make sure that this funding agency is open to learning and development. This improves not only the complex communication procedures with all actors involved but ensures also a successful programme management.

11. It sounds trivial but it isn't always in practice that enough money is earmarked and available when the MAP starts. Failing to ensure mid-term financial security hinders the start of the programme or the programme might start and projects can't be contracted in time after the evaluation. Thus policy makers should avoid short-termisms, secure mid term budgets and install multi-annual financial planning mechanisms as MAPs need (even more than traditional programmes) stability and perspective due to complex actor settings and due to the mid term "culture change" approach of most MAPs.

12. Think early of evaluation requirements: The design of the evaluation procedures should take place before the start of the programme. Evaluators and evaluation users need to be clear about which questions in which breadth, depth and how radical are to be asked; evaluation research can produce realistic information about an evaluation object only through the combined usage of various social science methods and indicators. The "objectivity" of evaluation results can only be approximately guaranteed by deliberately taking various, different actor perspectives into consideration. 


\section{SELECTION PROCEDURES OF MAPS}

\section{$5.1 \quad$ Introduction}

This chapter aims at presenting the important issues concerning selection procedures of MAPs. All but one of the MAPs involved in the MAP-TN and StarMAP use a competitive approach to the acquisition of projects, mainly fixed-term calls for proposals. We can observe a number of different approaches concerning the type of call for proposals, the number of stages involved in the overall selection process, the duration of the call and the arrangements used to evaluate the proposals submitted. Common to all the programmes is nevertheless the need to rely on sophisticated selection procedures and similar overall design principles, for MAPs much more than for "normal" RTDI programmes, as a variety of goals have to be addressed. The selection procedures are then tailored to the specific needs of the individual programmes, the stakeholders involved and the relevant features of the innovation system.

According to the members of the MAP-TN, selection procedures are one of the most critical aspects of programme implementation. They are discussed in two steps: (i) Call for proposals and (ii) Proposal evaluation

At each point in the process, one can see features that reflect the particularities of MAPs. In the following sections we will briefly describe important stages and features of MAP selection procedures, relying on experiences of the MAP-TN participating organisations and StarMAPexamples. Not all the issues are strictly MAP specific. Nevertheless the sum of the elements in the selection procedures show a clear MAP specificity and a number of characteristics common to all MAPs involved.

\subsection{Calls for proposals}

All funding institutions and programmes in the RTDI policy field need at least some organised form of project selection to guarantee a minimum of transparency, comparability, fairness and value for taxpayers' money. Such minimum standards include a visible framework of rules and procedures, a formal entry gate with application forms and a review by neutral experts. Another reason for increased formalisation is that as knowledge economy and innovation have become increasingly into the spotlight of economic policy and subsequently public funding for RTDI has been increased, increased attention has also been paid on accountability and impact of public funding for RTDI.

In the last two decades we can observe a trend towards greater formalisation and more detailed rules and criteria, probably in part triggered by the high formal standards of the European RTD Framework Programmes. Some common practice is evolving all over Europe, forming a kind of stepping stone for opening up of national (and regional) RTDI funding programmes. While the MAP-TN sees benefits from this process of normalisation, concerns were expressed - and experienced even - with regard to the formalisation of programme rules. 
The conclusion is that we must search for an optimum level of bureaucracy that adds clear value and beware introducing so many rules as to cause MAPs to collapse under their own weight.

\subsubsection{Competitive approaches?}

The majority of the MAPs under review organise their selection procedures in the form of competitions with a clear structure, timetable and end-dates. Typically, a call is announced using a mixture of marketing media to maximise the number, variety and quality of the response.

All but one of the 16 programmes employ competitive approaches to the selection of projects ${ }^{22}$. This competitive approach is generally considered to be critical to goal attainment in what are large and complex programmes. While there is a basic legal requirement in many countries that applies to most if not all categories of RTDI programme, the MAPs have a specific interest in the incentives that derive from competitive approaches. In general, competition drives quality, and diversity in applications and project execution and these attributes are especially important within the context of MAPs, with the strategic goals, large size and multilateral nature.

\subsubsection{1 $\quad$ Advantages of competitive approaches}

All programmes in the MAP-TN quote the issue of safeguarding transparent and fair decision making. The underlying assumption is that scarce public resources have to be distributed selectively in order to make an impact and in a comprehensible way. The advantages for using competitive approaches include:

- Value for money through competition around solutions and prices;

- Increased choice for the policy actors;

- A number of programmes highlight the issue of capability building and the mobilisation of resources;

- The quest for more long-term research efforts, namely in industry;

Perhaps most important of all, the use of formal structures and high levels of disclosure and transparency will tend to protect the MAP and its portfolio of investments from the need to mirror short-term fluctuations in policy priorities. Moreover, if there are no transparent and competitive selection procedures, there is room for political manoeuvring, pork barrels, back doors and sellers of grand designs. There should be no doubt: the shadows of all four dangers mentioned are always present.

22 None of the MAP-TN programmes and StarMAP examples use other types of procurement mechanism, such as a restricted invitation to tender.The exception is the Norwegian TEFT programme, where candidates are selected by TEFT technology attachés on the basis of their personal knowledge and in consultation with the Norwegian Industrial and Regional Development Fund (SND). 


\subsubsection{2 $\quad$ Risks of competitive approaches}

While competitive approaches have advantages, there are risks too. The need to observe highly formalised procedures and timing to permit fair competition can result in a level of rigidity that may lead to a dilution of social or economic benefits which were discussed broadly in MAP-TN, other issues that have been observed from time to time include:

- Where 'competition' becomes too intense, with large numbers of applicants competing for a small number of grants, proposers may tend to manage this burden by increasing numbers of proposals and downgrading the quality of proposals. Taken to extremes, this can lead to uniformity in applications and suppress diversity in subjects, methods and risk profiles at the portfolio level, with a further implication that the beneficiaries might be pressed into shorterterm activities and under-investment in people and infrastructure.

- There is a danger of competitive approaches producing imbalance in the portfolio with the distribution of investments reflecting established capabilities and strengths. In the case of MAP-TN members, most have managed this risk by pre-selecting areas of strategic importance and committing to support only one nationally-significant investment in each strategic area (e.g. the Competence Centres programmes).

- There is a risk of excluding groups that might reasonably expect special assistance, such as those working in emerging and inter-disciplinary fields, although this may be dealt with through careful selection criteria.

- Do the best apply? Complex bureaucratic procedures and low success rates can be a disincentive to leading industrialists and researchers, and particularly where they have access to other research funds and state assistance (national or international) with simpler requirements and better prospects.

- Competition demands a rigorous and reliable proposal evaluation procedure even though MAPs must employ multiple criteria, mostly subjective, to choose between the long-term potential of a proposed action of possibly dissimilar proposals;

- Given the fact that perhaps $50 \%$ of proposals will need to be rejected there is a need to reflect on the legitimacy of such a social burden (including costs) and the consequences of widespread disappointment.

These risks are general to all MAPs and can be answered partly by in-built flexibility like differentiated sub-calls (with particular criteria), by giving assistance to newcomers and generally to stick to the above mentioned rules and ethics. 
The UK Partners in Innovation scheme (PII)- Evolving sub-programmes to start new trajectories.

PII shows within the programme framework three sub-programmes. PII OPEN is directed to all research active organisations in the UK and has a broad project portfolio on the basis of open competitions. With this instrument the quality of proposals from the construction sector could be raised, to a lesser extent also the number of innovative firms. PII FOCUS is a managed competition for priority areas. In this subprogramme excellence could be developed in key areas, accompanied by a certain visibility of efforts. PII PROGRAMMES as a new element tries to attract large consortia (similar to Networks of Excellence) within a given problem / area. All three sub-programmes have similar but adapted selection procedures based on competitive two stage application procedures and evaluation panels. There are five common sets of criteria. As capability building and problem-orientation are key for this programme, PII had to differentiate it's portfolio to reach also actors who did not participate in the first place. This had been by the way also an important argument to come forward with competitive procedures. In the course of the programme there was stakeholder pressure to have specific and un-anticipated issues supported likewise, so the programme was split into PII OPEN and PII FOCUS in 1998. In 2002 PII PROGRAMME was introduced to ensure a sufficient scale of investment by a critical number of stakeholders in strategic areas. There is even a fourth, ministry-led fast track scheme to fund very small projects. While different policy issues can be handled within PII, this programme is still considered, managed and presented as one MAP.

Some agencies fund losers through other programmes, given the fact that often the quality of MAP proposals can be high without fitting to the structural requests of the specific programme. While there are many risks that have to be managed, all MAP managers believe that the advantages of the competitive approach, outweighs the negative implications. The exception again is $\mathrm{NO}$ as the main aim of TEFT is to stimulate research in SMEs. To achieve this aim a technology attaché visits promising companies and carries out interviews and a technology audit. After that the attaché writes a pilot project report and links companies with appropriate research institutes. That's the whole unbureaucratic selection procedure (which would not at all be typical for a MAP, but note that TEFT is none). 
The Austrian K plus programme: The pro's and con's of competitions as a deliberate instrument of policy change

In Austria, K plus - started in 1997/98 - was the first large-scale funding programme to use competitive selection procedures. Learning from Sweden, Australia and other countries, the Austrian policy makers in charge deliberately chose this instrument to change the research culture and also to bring new elements of funding culture into the Austrian innovation system - namely when granting large projects. To ease the policy change, $\mathrm{K}$ plus started with a pilot scheme, inviting only a limited number of consortia to apply. Already one year later the first open competition started. In the following years two interesting developments could be observed. Parallel to the $\mathrm{K}$ plus programme a vast number of other funding programmes were designed as competitions. On the other hand a second, more industry- and networkoriented Competence Centre programme was installed by another ministry. This second programme called $\mathrm{K}$ ind / $\mathrm{K}$ net does not use competitive selection procedures but individual negotiations with strong industry consortia. This development was only partly a surprise for those involved.

The $\mathrm{K}$ plus programme tries to manage the risks mentioned as follows: Rigidity is softened by subsequent calls and managerial flexibility on all levels. Danger of uniformity is answered by individual solutions for individual consortia, as long as they stay within the general $\mathrm{K}$ plus framework. Overinvestment in preparations and creation of losers is a most difficult issue which can partly be answered by an open information policy, a two-step procedure and help to unsuccessful consortia to develop their ideas, perhaps with other funding sources. The exclusion of actors - along quality criteria - is an explicit goal of the programme. Not all actors around shall get some funding. Ranking problems finally are solved by a matrix approach through the combination of "scientific" and "industrial/managerial" ranking.

\subsubsection{Open or fixed-term calls for proposals}

While in fixed-term calls proposals have to be submitted at a fixed date latest and are all evaluated against each other, in open calls there is continuously the opportunity of submission; evaluation takes place in fixed periods or depending on the number of proposals received.

It appears that MAPs use one type of call for proposals rather than several, for reasons of simplicity and consistency of administration. Just two of the 15 MAPs make use of a mixture of types of call for proposals, reflecting the different needs of the particular funding stream. So, for example, an annual, fixed-term call for proposals is employed within the strand of the programme seeking to launch research networks of national scale and significance, while a continuously open call is used for the small-grants fund directed to capture innovation within businesses. Within fixed term calls there are further differentiations: (i) Annual or regular fixed term calls (UK, NL), (ii) one or a series of fixed term calls with indicating exactly when the next call will take place (SE, AT), but with no regular "timetable".

Generally, MAPs prefer fixed-term calls over calls that are open continuously, as a way to generate competition among applicants (and as an incentive to prepare better-quality proposals) on the one hand and to simplify programme administration on the other. The advantages and risks of open and fixed-term calls are displayed in the following exhibits. 
Exhibit 8: Open calls for proposals

\begin{tabular}{|c|c|}
\hline Advantages & Risks \\
\hline $\begin{array}{l}\text { Permits programmes to respond to emerging and } \\
\text { innovative opportunities in a timely fashion, which may be } \\
\text { of especial interest to business or occasional applicants }\end{array}$ & $\begin{array}{l}\text { Continuously open calls will tend to reduce the numbers of } \\
\text { proposals available to be considered at any one point in } \\
\text { time and, as a result, may weaken competition to a level } \\
\text { that is undesirable }\end{array}$ \\
\hline $\begin{array}{l}\text { Reduces the potential for calls to generate peaks and } \\
\text { troughs in workload of both applicants and the MAP } \\
\text { management unit }\end{array}$ & $\begin{array}{l}\text { May reduce volumes of applications to the point that the } \\
\text { programme management cannot commission an } \\
\text { appropriate spread of projects }\end{array}$ \\
\hline & $\begin{array}{l}\text { Reduces the opportunity for a programme to involve } \\
\text { eminent individuals from across the stakeholder } \\
\text { community in evaluations or high-level supervisory body }\end{array}$ \\
\hline
\end{tabular}

\section{Exhibit 9: $\quad$ Fixed term calls for proposals}

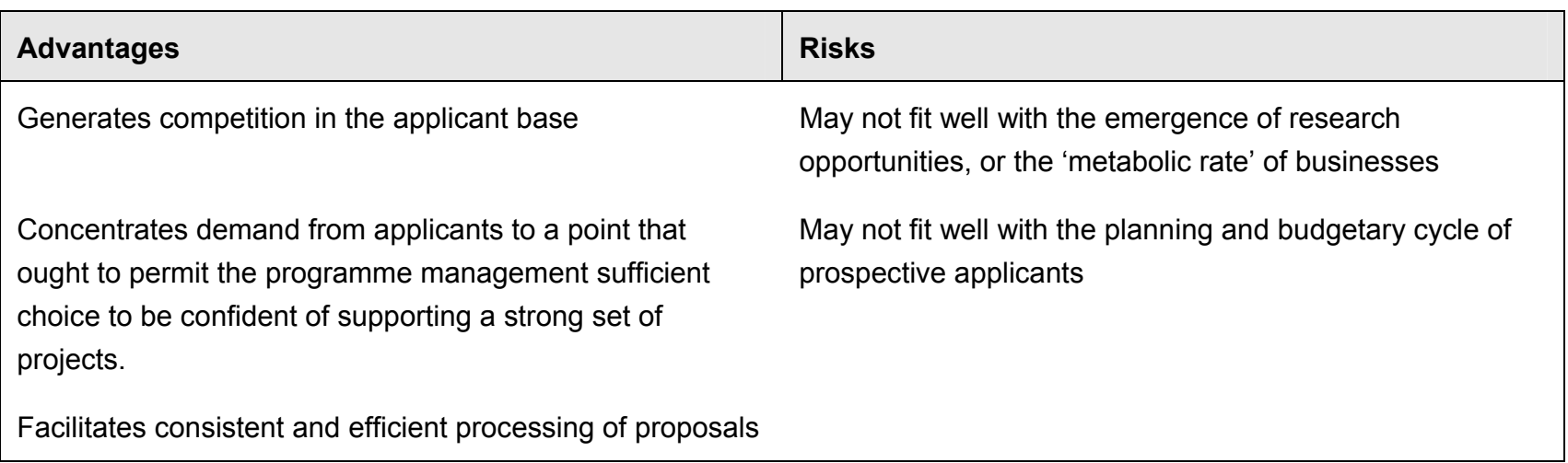

\subsubsection{One or two-stage application procedure?}

In a two-stage procedure applicants have in the first stage the opportunity to express interest by letters of interest, proposal outlines etc. in participating in the call. After an evaluation of those "expressions of interest" which are organised very differently in the examples investigated a subset of interested proposers is chosen for submitting a full proposal. On the other hand, in a one-stage application there is no such intermediate evaluation and/or choice, i.e. all applicants submit full proposals at the deadline of the call.

Given a free hand, participants in MAP-TN expressed a strong preference for using the twostage application procedure because it permits a much greater degree of control over the quality of the individual projects, which is particularly important in Competence Centre MAPs, where there may be just one award or investment in each strategic area. Another advantage which has to be used with caution is portfolio building, perhaps of more importance to the thematic and cluster MAPs. Another case when it seems to be better to use the two stage application procedure (again to be handled with care) is when it is foreseen or expected that potential applicants do not prepare joint proposals, the first stage feedback can be used to identify potential consortia and recommend joint preparation in stage two. Moreover, increased management costs are offset by stronger programme performance on goal attainment and return on investment. 
While there was a general preference for two-stage procedures, in practice only six of the 15 MAPs use a two-stage application procedure, with a formal evaluation of both the outline and full proposals (AT, AU, SE, HU, EE, CA), while seven MAPs use a one-stage procedure. Three others (the cluster MAPs) used both a two-stage application procedure and a one-stage procedure, depending on the nature of the sub-programme (SL, FI, UK).

The number of stages is not directly linked to the size of the applications and projects, though a two-stage procedure is used more often in large Competence Centre (and Network) programmes (AT, SE, HU, EE, CA). Especially the Swedish and Austrian MAPs are content with this solution, reducing workloads on both sides and allowing the funding agency to send signals to the field indicating the strategic direction in which they would hope the proposal could be developed. Industry needs time to give long term commitments and generally large consortia are not ready to start overnight. So a first warm-up round based on outlines and Letters of Intent is seen as a good procedure. There is one cluster programmes with a two-stage approach (UK), while others (SL, ES, IE, FR, NL) use a one-stage selection procedure. The latter is also feasible for Competence Centre programmes (DE), partly because of the possibility to install some feedback elements in a one-stage selection procedure.

Box 14

The Slovenian Industrial Clusters Development (Toolmakers): switching between one and twostage procedures

The Slovenian Industrial Clusters Development Programme uses a two-stage application procedure from time-to-time where the programme management unit considers there to be an especially large number of prospective applicants. The two-stage process permits the programme to minimise the cost of bidding to ultimately unsuccessful applicants.

\section{Box 15}

Programme and UK Partners in Innovation Programme: switching between one and two-stage procedures

The UK Partners in Innovation programme uses a two-stage application in most instances, but does operate a single-stage application procedure for its small grants-funding stream (which amounts to around $5 \%$ of its annual spend). In this case, it is a rule of thumb that the effort devoted to the ex ante evaluation of proposals should be proportionate to the size of the grants being awarded. The ministry is content for an advisory group to selectively award grants of say EUR 10,000 based on a single-pass evaluation of a batch of five-page proposals while insisting on a three-stage application process - and a 100-page proposal - for grants over EUR 1 million.

When looked at in aggregate, the data do not reveal any strong patterns with regard to the use of a one-stage or two-stage application procedure, and do not provide any indication as to whether one route might be preferred to the other and under what circumstances. When looked at a little more closely, however the case material suggests there may be several factors that inform the choice of single- or multi-stage evaluation, which include:

- The size of the awards envisaged, with two-stage applications tending to be preferred for programmes awarding larger grants. 
- The level of competition for awards, with two-stage applications tending to be preferred where demand far outweighs the available funds. In this case, one is seeking to be efficient in finding the best proposals among the many and to manage the burden of not being successful.

- The openness of the call in thematic terms, with the more open calls tending to use twostage application procedures wherein the first round is a 'competition of ideas' while the second round is a competition of feasibility and value.

Historical precedent seems to be rather more of a determining factor in the choices made however, with these other factors perhaps tending to colour an administration's thinking rather than form it. The advantages and risks of one and two-stage selection processes, as well as the advice on when to use one or the other, are summarised in the following exhibits.

\section{Exhibit 10: One-stage application process}

\begin{tabular}{|l|l|}
\hline Advantages & Risks \\
\hline Speed of selection process (average 1-2 months) & $\begin{array}{l}\text { Concentrate demand on leading consortia, entrance } \\
\text { barrier for "newcomers" } \\
\text { Tends to be simple to administer }\end{array}$ \\
Tends to involve lower costs for programme management & $\begin{array}{l}\text { High rate of rejection } \\
\text { Tends to increase the costs of bidding for unsuccessful } \\
\text { proposers }\end{array}$ \\
\hline
\end{tabular}

When to use: (i) Where requirements can be more closely specified, (ii) Where grants are small, (iii) Where programme is small, (iv) Where speed of response is critical e.g. to attracting applications from consortia involving SMEs.

\section{Exhibit 11: Two-stage application process}

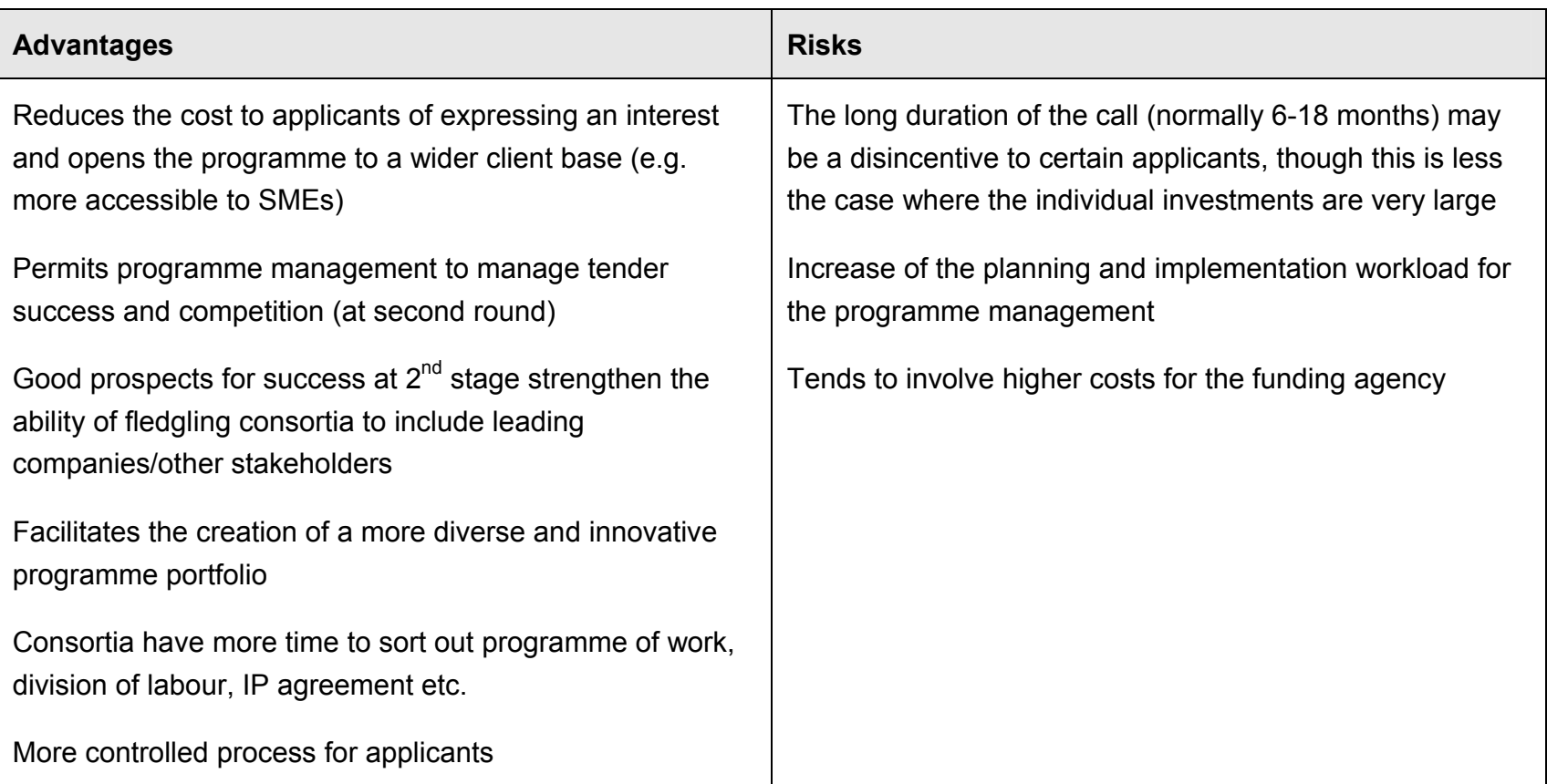


When to use: (i) Programmes with complex, multiple goals, (ii) Programmes with large projects, (iii) Programmes with large consortia or networks, (iv) Where the available budget is rather small when compared with the volume of demand, to avoid producing a large number of users and high costs for the losers, (v) Where anticipated projects are very large, and involve a highdegree of concentration of funding in a given field, with grave consequences for unsuccessful consortia, (vi) Where SMEs are a major focus

The time required from deadlines for proposals to decision varies among MAPs. The large Swedish Competence Centres Programme call, implemented through a two-stage selection, took more than 18 months to conclude. Hungary had a six month run through process for similar centres and Germany applied the same amount of time with its one stage selection. Both countries employ consultants to run expert evaluation panels, recompense the panel members and have arrangements that tend to be quicker to run than classical peer review with its reliance on academic support and goodwill. For smaller projects in cluster-like MAPs (UK, SL, BE) the typical time-scale is three months for writing and three to four months for assessing the proposal. The differences are explained in part by programme lifecycles. The Swedish case was the time taken for the first call of what was a pioneer in the Competence Centre movement, where in other cases (e.g. BE) it is a steady-state time for a programme that has been running for some years.

The time needed for selection depends on several variables, out of which some are listed as examples: (i) The number of stages in the competition, (ii) The arrangements chosen for the proposal evaluation process.

Two-tier evaluation involves both an evaluation committee (experts' panel, high-level group comprising major stakeholders) and peer review (where domain specialists provide proposalspecific evaluations). Obviously those MAPs operating with two-tier evaluation require more time, especially if the domain specialists provide written information for the experts' panel in time as additional information for their judgement. Evaluation processes relying on recommendations of the experts' panel without peer review take less time.

- Equally, one might see longer duration processes where MAPs are providing proposalspecific written feedback to the applicants, facilitates learning and trust among programme management team and applicants, rather than simply notifying people about decisions;

- The size and complexity of the proposals anticipated, with MAPs that foresee funding a small number of large proposals tending to take longer to process those proposals (bigger proposals demand higher levels of scrutiny).

Overall, the single stage calls tend to be cheaper to administer and faster to conclude, which is rather attractive to financial controllers and to industrial participants. By contrast the two-stage procedure may cost more to administer and take longer to conclude, however there is greater opportunity for strengthening work programmes and consortia through feedback/learning between rounds. 


\section{The Swedish Competence Centres: "We did it in one big bang!"}

As many other MAPs the Swedish CC programme is a deliberate attempt to change research and cooperation culture in the innovation system (Hjorth 1998). The proposed wide and selective selection procedure was one main argument to get the programme granted within the 1993 research bill. While the principle and the thrust of the programme were soon accepted on the policy level, financing proved difficult and nearly led to over-coordination with other funding sources. These problems in the early stage brought up considerations to start with selected technological areas, but soon it was decided by NUTEK to come forward with a broad approach to address all high quality groups and fields in Sweden. So a real nationwide competition between most technologies and industrial sectors took place. The programme was even considered to be a kind of implicit, hands-on foresight programme. Competitive bidding for large-scale cooperative consortia was "learnt" in preceding NUTEK materials research consortia around 1990.

The two-stage selection procedure was the result of a learning experience with the US NSF Engineering Research Centres programme. The special feature of the Swedish process was that even those not invited to enter the second stage could do so - but without receiving a planning grant. The immense workload for all the participants can be illustrated by the sheer numbers: 326 applications in the first stage, 61 planning grants, 117 final proposals, positive funding decision for 30 centres. Eight expert panels (one of them being the overarching central committee giving funding recommendations to NUTEK) and 20 NUTEK programme managers dealt with this process. Note that in this selection procedure no classical external peer review took place. VINNOVA, NUTEK's successor, now considers a new selection round for CCs, but maybe not on such a large scale at a time.

\subsubsection{Electronic submission of proposals}

Many public administrations have pressed programme management units to implement electronic submission of proposals as part of their wider obligations to meet targets on moving to paperless environments. It is assumed that switching to electronic proposals will improve management efficiency and throughput rates.

In practice, MAPs have had mixed success with this technology and challenge the applicability of the general assumption that electronic submission will yield the kind of benefits mentioned above. Pure electronic submission is mandatory in just two countries at present (ES and HU). The UK MAP uses fully digital communication at the outline proposal stage and operates a voluntary system at the full proposal stage; the evaluators however continue to demand the large, complex proposals be delivered in hard copy. While Austria uses electronic submission only as a backup and Sweden has withdrawn from its attempts to make a success of a paperless process with the interoperability and security problems being deemed to outweigh the benefits. 
Exhibit 12: Electronic submission

\begin{tabular}{|l|l|}
\hline Advantages & Risks \\
\hline $\begin{array}{l}\text { Reduces the need for the management team to re-key } \\
\text { data and avoids the introduction of mistakes by } \\
\text { administrators }\end{array}$ & $\begin{array}{l}\text { Evaluators tend to prefer to work with hard copy, as it is } \\
\text { easier to work with, and it can be worked with in more } \\
\text { places }\end{array}$ \\
$\begin{array}{l}\text { Reduces the time required to receive, log and compile } \\
\text { proposals }\end{array}$ & $\begin{array}{l}\text { Database and servers receiving applications may struggle } \\
\text { to cope with peaks in submission }\end{array}$ \\
$\begin{array}{l}\text { Helps evaluators to perform faster and more effective } \\
\text { searches on multiple proposals }\end{array}$ & $\begin{array}{l}\text { Application software may not be accessible to all } \\
\text { prospective applicants, as they may not possess the IT } \\
\text { equipment and skills necessary }\end{array}$ \\
$\begin{array}{l}\text { A digital archive enables evaluators to access past } \\
\text { proposals to check additionality }\end{array}$ & $\begin{array}{l}\text { Interoperability problems can 'lose' and or corrupt } \\
\text { proposals, damaging applicants chances of success } \\
\begin{array}{l}\text { A digital archive provides managers with easier access to } \\
\text { past funding decisions }\end{array}\end{array}$ \\
$\begin{array}{l}\text { A digital archive requires less storage space at expensive } \\
\text { premises }\end{array}$ & $\begin{array}{l}\text { Security technology is complex and unstable } \\
\text { shere is uncertainty over liability and compensation for }\end{array}$ \\
\hline
\end{tabular}

When to use: (i) Where there is a need to process a large volume of proposals in a short space of time; (ii) if the system is proven to work reliably, can be used by people across many organisations and with widely different IT ability; (iii) net based password secured applications are probably the future in paperless submission and assessment of proposals. This is possible as soon as experts have learned to work without paper. The added value of this is security and confidentiality (controlled access to information), possibility to work between several organisations and geographical locations, etc.

\section{Exhibit 13: Paper submission}

\begin{tabular}{|l|l|}
\hline Advantages & Risks \\
\hline $\begin{array}{l}\text { High levels of reliability for administrators, evaluators and } \\
\text { applicants, because it has been in use for decades }\end{array}$ & $\begin{array}{l}\text { Make it harder for administrators and evaluators to deal } \\
\text { with each call for proposals from an informed position } \\
\text { about previous calls }\end{array}$ \\
$\begin{array}{l}\text { Most evaluators find hard copies more legible and more } \\
\text { flexible (in terms of where the evaluations are performed) }\end{array}$ & $\begin{array}{l}\text { Programme management units descend into paper } \\
\text { jungles, with no room for people }\end{array}$ \\
\hline
\end{tabular}

When to use: Everywhere, until the substitute electronic system has been fully proven

Electronic submission - and digital systems generally - hold out the promise of important advances that will be of especial utility for MAPs, given their complexity and learning nature. Databases/archives of proposals and projects can be used both by the administrators and programme evaluators to monitor and research patterns of behaviour among applicants and contractors. The electronic submission permits the evaluators to make more informed decisions about current requests for funding, while the administrators have a more solid basis for deciding about research priorities and the division of labour between funding bodies/programmes. 
In practice, these technologies have not proven to be easy to implement and offer much lower levels of reliability as compared with paper-based systems, when considered at the system level. As a result, these advanced systems can erode trust/respect for the MAP and the programme management unit among applicants as well as incurring additional costs; the opposite of what they are supposed to offer. In practice, one can expect to see a gradual transition to electronic submission as the technology becomes more robust and processes become more appropriate.

\subsubsection{Support to applicants}

All programmes covered by the MAP-TN and StarMAP actively provide information within the limits of fair procedures (e.g. the provision of information and advice stops shortly before the deadline for the call in UK), and most, though not all, provide direct support to applicants too. Some MAPs show active matchmaking (DE, BE). Others arrange briefing meetings for applicants, which present the programme and give people an opportunity to seek clarification regarding application procedures and selection processes (AT, EE, FR). Competence Centre programmes with a two-stage process give planning grants in the second stage (AT, SE, HU, EE), other programmes sometimes have funds for feasibility studies (IE, UK). Financial support to applicants is provided to offset the cost of developing comprehensive proposals for large projects or national centres of excellence, which would be expected to operate over 5-7 years.

MAPs offer extensive written information (due to complex goals and procedures) on all aspects of the programme: its goals, financing, selection procedures and operational arrangements. They also provide standard forms for making an application, for consortium agreements, for monitoring, et cetera. The majority have an 'open-door policy', with applicants being invited to approach administrators to discuss proposal ideas and needs (e.g. help with identification of partners). Those with a two-stage selection procedure offer written feedback on first-round proposals, with a view to assisting applicants with the orientation of proposed work and its methodological robustness.

MAPs provide rather more support to applicants than is normal for other RTDI programmes managed by the same ministry or agency. This reflects the complexity of typical MAP 'actions' and the desire on the part of the administrators to: (i) attract a sufficient number of strong proposals; (ii) increase the prospects of programme success and (iii) reduce the potential to waste resources. 


\subsection{Proposal evaluation - particular challenges; the systemic view}

MAPs tend to be aligned against socio-economic goals the realisation of which may be of a rather more challenging and uncertain nature than is the case for classic RTDI funding programmes. As such, proposed projects offer to orchestrate a variety of social actors with different and complementary backgrounds/disciplines to work together to achieve a specific impact several years in the future. It is assumed that MAPs will use a multi-stage call for proposals and a competitive selection procedure, the objective of the process being to build a portfolio of projects that offers the greatest likelihood of securing the MAP mission. Portfolio building should not be a taboo, but if misunderstood it can easily be. Also here the use of an impact model and open targeted calls based on portfolio analysis and/or the original MAP rationale can be used to avoid misunderstandings related to portfolio building, using transparent rules. For example, cluster MAPs can not lead into networks and clusters if key linkages between key actors are not developed. If such proposals are not received or they are not successful in the competition, targeted calls are a way to encourage and ensure them.

Some considerations should be taken into account when building portfolios:

- Portfolio building can be a taboo when open calls are running;

- Portfolio-building can be necessary when multi-goals are to be found in the MAP and many applicants submit proposals;

- Portfolio-building can be very explicit if it is goal in itself;

- It can happen implicit if there is a jury/committee to come forward with a nice basket of projects (but danger to make politics and the field happy);

- Please note that strict transparency rules have to be applied when building portfolios.

Note that the number of MAPs explicitly rule out the possibility of portfolio-building and rely on a strict bottom-up process even if strategic allocations are the result of the selection procedure (e.g. in the AT K plus programme)

The 16 programmes under review strongly suggest that any organisation wishing to operate a MAP successfully will need to arrange a rather different process as compared with more traditional RTDI programmes when it comes to the ex ante evaluation of proposals.

MAPs generally: (i) focus on socio-economic objective/s and evaluation tests give most weight to appraisal of a proposed project's likely impact on the problem/target audience; (ii) draw from, and address, both the scientific and the industrial world. Therefore ex ante evaluation procedures from both worlds have to be employed, combining for example scientific peer review with quantitative investment appraisal techniques; (iii) in addition to that societal evaluation criteria could be used where appropriate; (iv) manage portfolios rather than single projects encircling a problem with multiple initiatives employing multiple measures - with final decisions on centres or projects tending to be made at the level of the 'set' or the programme. 
The Finnish Technology Programmes: Portfolio building

One way of tackling the problem of unbalanced portfolio: Targeted competitions are used, i.e. call for proposals of specific types of projects. Tekes has used this in context of technology programmes, when a programme steering group has identified a balance problem they have launched a focussed/targeted call for specific types of projects. The call is still competitive and open for everyone, but additional criteria are used to make sure that the new projects balance the programme portfolio. The approach Tekes has frequently used to reduce undue burden for project preparation and rejection is the use of two step application procedure (two step calls for proposals).

In the following two sections, we review the issues around MAPs' ex ante evaluation processes and evaluation criteria.

\subsubsection{MAP proposal evaluation processes}

Typically, MAP evaluators are identified by the MAP management unit, or its management support contractors, often from an established database of experts using a combination of competence, experience and location. The independence of experts is important and in fact, 14 of the 16 programmes studied use external expertise at the heart of their ex ante evaluation process (except $\mathrm{FI}$ and $\mathrm{NO}$ ).

Most MAPs use a modified form of the classical peer review, whereby each proposal is considered by several evaluators with a range of competencies and experiences rather than a group of leading scientists working in the same sub-field as the applicant. Such a heterogeneous group provides the management unit with the ability to test each proposal on several markedly different dimensions, for example: (i) the relative importance of the proposed outcomes/impacts; (ii) the additionality of those intended benefits over that which might be expected to arise without the intervention, (iii) the methodological robustness of the proposed work plan; (iv) the track record of the proposed consortium and its project director; (v) the value for money offered by an investment as compared with other fundable options.

The care and resources devoted to the process tend to be proportionate to the size and complexity of the MAP in question, as well as the particularities of the programme objectives. These are the common procedures of ex ante evaluation:

- Compliance checks (eligibility checks) made by the MAP programme management unit or its management support contractors for example on the eligibility of particular categories of participants, or the funding requested, for a given form of support;

- Expert assessment (peers, domain experts) of each proposal in terms of its 'additionality' and its 'feasibility,' with written evaluations - scores, comments, recommendations provided to MAP management unit for compilation and distribution to the MAP high-level evaluation group (experts panel);

- Presentations to or site visits by high-level evaluation/advisory groups 
- High-level evaluation/advisory group or groups with responsibility for recommending to the budget holder the sub-set of proposals from the whole call - or thematic area - deemed to offer the best chance of delivering on the MAP mission. Such groups or juries rely on the results of the individual reviews and have an overview on all proposals while judging along strategic criteria.

If written statements by experts from the field (peers) are included we can describe it as extended peer review, including site visits, organisational and economic feasibility, a matrix evaluation approach and a final expert jury (AT, AU, CA; to some degree planned in EE). Similar approaches include technology experts drawn from the agencies' databases (ES, UK). A pure jury / experts panel approach is installed in a number of countries, ranging from one expert panel (DE, HU) to a number of co-ordinated disciplinary panels (SE). See also the considerations on ex ante evaluation in chapter 7 .

It is interesting to see how far foreign experts are included. For the above mentioned panels mostly national experts were chosen (HU, DE, SE, FR, NL in AT the final expert jury), but all of them had some foreign element included. Especially small countries face difficult trade-offs between excellence, bias, partisanship and knowledge about the system. In the Austrian case, the six scientific peers per centre application are all foreign, while the economic feasibility is written by Austrian experts. Estonia also plans a foreign peer review process. In AT the foreign peers are selected by the Austrian Science Fund, i.e. the national research council, as a contractual partner of the funding agency TIG. Overseas, in Australia and in Canada, also foreign evaluators are used to avoid a bias.

In MAPs granting smaller projects, it is mostly the agencies' database (UK, ES). If large centres are to be granted and panels are in charge of the selection recommendations, it is either the strong agency (SE, EE) or the responsible ministry (HU, DE) who selects the peers.

IE and FR use external evaluators only on a case by case basis. FI with Tekes is an exception of the rule of using external experts in ex ante evaluation, unless the programme planning/steering group is considered to act as a panel of external experts. However, the planning/steering group consists of stakeholders, so the only independent experts in ex ante evaluation are Tekes experts. In Norway the Technology Attaché is responsible for setting up and finally recommending projects for funding.

Anonymous peer review is another interesting question with a broad range of answers. While some countries adhere to non-anonymous procedures due to funding traditions (SE, CA), size of country (IE) or the concept of the programme (HU, ES), other MAPs have the principle of anonymous reviewing (SL). In AT the $\mathrm{K}$ plus peers stay anonymous until the stage of the visiting committee. The issue of confidentiality is clearer: Most MAPs regard all relevant information as confidential, varying degrees are the result of different national public service information policies.

Evaluators in MAP selection procedures usually are rewarded, because they face a complex task and the issue is not a purely academic one. In three of our MAPs, this is not the case and seen as an honour (SE, FR, AU). In other countries the decision to pay was also fuelled by strict timetables (AT, UK). 


\section{Box 18}

\section{The German CC Nanotechnology: A mixed expert board approach}

This German MAP gives support to centres without granting large funds for RTD work. Nevertheless the German "Nanolandscape" is partly shaped by the programme because of the label of excellence that comes along with Competence Centre funding. To choose between the $\mathrm{CC}$ proposals a jury of nine experts was installed by BMBF as responsible ministry, including two experts from German scientific institutions, one from the German finance sector, three from German industry; a Swiss and an American Nanoexpert and a Dutch technology policy specialist completed the panel.

Those nine specialists got the proposals from the responsible project agencies. The latter managed the preparatory stages of the programme, gave advice to the consortia and collected the proposals. After an eligibility check, the proposals were given to the expert jury. They discussed the proposals and gave recommendations. The final funding decisions were taken by the ministry. This is an example for a rather low-intensity external expert use, contrary to a full scale peer review model. This approach is adapted to the needs of the programme as individual RTD projects and work programmes of these centres are subject to other ex ante evaluation procedures.

\subsubsection{Ex ante evaluation criteria}

In all funding programmes clear ex ante evaluation criteria have to be in place well in advance. They have to allow for differentiation and evaluation. They are perhaps the most important signals a funding body sends to the innovation system or a certain actor set. MAPs need complex ex ante criteria as they try to influence whole sub-sets of an innovation system. All MAPs present their explicit evaluation criteria to potential applicants in advance. This is almost a requirement when designing any funding programme. The range of criteria partly depends on the type of MAP. While formal eligibility criteria like timeliness and completeness are common to all programmes, qualitative criteria vary. Track record is important in nearly all the programmes investigated. The scientific quality of the proposal is central to a number of MAPs (AT, EE, HU, SE, AU, CA). 
The Hungarian KKK programme: Strict Criteria in drifting policy frameworks.

While the Hungarian programme faced some difficult times regarding responsibilities, overall political support and "re-integration" of agency structures into the ministry, two stable factors helped - inter alia to deliver excellent results of the selection procedure. The first was the highly motivated KKK management committee, the second a clearly formulated and rather strict set of funding criteria providing orientation to all parties involved. These criteria included a number of workable sub-sets:

\section{"Evaluating the objective of the CRC":}

Extent of industry partner inclusion in goal-setting, ability to satisfy user needs and relevance of academic institutions in this respect, work programme reflecting industry orientation, perspectives for social and economic benefits (these criteria proved to be the most difficult ones).

"Professional values and feasibility of the research programme":

Quality of work programme and chance for breakthroughs, relation programme - goals, feasibility, competences of key personnel, appropriateness of infrastructure, chances to implement strategy, IPR policy.

"Relevance of the educational and training programme":

Quality of training also regarding industry needs, $\mathrm{PhD}$ programme, vehicle for technology transfer.

"Cooperation programme of partners":

Academic commitments, industrial commitments, commitments regarding long term strategy.

"Organisational structure":

Appropriateness of resources, quality of proposed organisation, excellent director, clear roles.

"The role of $\mathrm{CC}$ within Higher Education Institution":

CC programme fitting to university.

"Performance indicators":

Acceptable internal evaluation procedures, meaningful indicators.

Some of these criteria are quantitative, some qualitative. The committee members used them as a basis for a scoring system, complemented by verbal comments. On both stages there were individual scorings, feedback within the committee and meetings to discuss the results. This system is elaborate but helped the KKK committee to rank the proposals.

Socio-economic aspects can be drawn broadly (AT, HU) or just include the question of mid-term industrial benefits (SE). They can be more general or be linked to indicators like propensity to form spin-off companies or to generate innovative products (DE, UK). The issue of good management and planning concepts is especially important when centres are to be established (SE, AT, EE, HU, AU, CA) and / or when there is a strong "value for money culture" implanted in the national system (UK, but see also ES). Another issue of Competence Centres is the question about system renewal and changing research culture (SE, AT, AU, CA).

Quality of existing networks can be an important criterion for centre applications (DE), as the long-term prospects (DE, AT, AU, CA) and the build-up of international links (SE, AT, FI, NL). 
The quality of education and training is also a key requirement of Competence Centres (DE, SE, AT, EE, HU, AU, CA). MAPs funding single projects ask for quality and innovation of this project and about potentials for commercialisation (ES).

\subsection{Lessons from the MAP-TN and StarMAP}

\subsubsection{Good practice in the selection of projects}

The procedures employed by members of the MAP Thematic Network do converge at several points at what we might call preferred practices, which include:

1. Widely publicised calls for proposals, with a fixed deadline, rather than targeted invitations: This encourages new blood, new alliances and innovation, increases number of applications, intensifies competition and helps driving up quality and value for money.

2. A two-stage application process where programmes are directed to supporting large, complex 'actions' and demand for awards far outweighs the number of (large) awards on offer. Where the scope of the programme mission is broad, a first round permits something approaching a 'competition of ideas'.

3. A different approach compared with conventional programmes, with a much greater emphasis on interaction with, and coaching of, applicants around investments that have major socio-economic objectives, seeking a significant change in capability and or behaviour. There should be also a greater emphasis on transparency and fairness to secure trust, where the individual investments are to be so large and selective.

4. Full and varied support to prospective programme participants including comprehensive programme literature, in print and online; conferences and briefing sessions for prospective participants; and an open-door policy with applicants able to visit programme managers. Support and interaction intensifies as the procurement process moves from the first to the second application stage. In some cases, administrators (and evaluators) will visit prospective research networks to discuss proposals while in other cases, administrations make available proposal development grants.

5. A two-tiered proposal evaluation system with an overarching high level group / jury selecting the best projects based on a set of 'technical' reviews considered against the MAP mission and budget. Technical reviews are provided by (panels of) reviewers (peers and other experts) considering sets of proposals grouped by theme or topic area.

6. Multiple, and sometimes soft evaluation criteria, including: (i) Quality and appropriateness of project 'inputs,' from methods, to resources, to partnerships; (ii) The nature, extent and attractiveness of the promised benefits, from the perspective of the MAP mission and priorities; (iii) The likelihood that the proposal will secure the promised impacts; (iv) The value for money of one proposal as compared with all others 
7. To use formal evaluation criteria and record the assessments of reviewers, scoring and commenting on each proposal. Several MAP-TN members provide evaluators with a full set of evaluation reports and spreadsheet information on bids and budgets, by MAP priority. Formal evaluation criteria offer structure and discipline and provide a basis for discussion among reviewers and increase likelihood of consensus as to which set of proposals is deemed most likely to deliver the MAP goals. Furthermore, they ensure the programme management team has access to intelligence based on consistent and careful assessment of all proposals on key questions for the MAP.

\subsubsection{Critical success factors}

Attracting and identifying the best projects is critical to any RTDI programme, however MAPs are perhaps even more exposed to this issue with their complex actor settings, various measures and finally the broad range of goals including goals on the meso level. However, be aware of 'goal overload' and goals too broadly defined.

All programmes described in the MAP-TN try to be clear and open about their goals, procedures and decision-making structures. Acceptance of the ex ante evaluation process by winners and losers is an explicit success factor in a number of countries.

Feedback to failed applicants ranges from informal information to highly formalised information policies and even referral to other possible funding sources. However, only a few programmes measure customer satisfaction as a performance indicator for the programme (UK). Another MAP-specific feature that appears to be critical to success is the consultation with stakeholders and applicants in parallel with the call for proposals. Often, we also find that MAPs have a relationship with applicants in terms of the volume/quality of support given in the run up to submitting proposals for what are large, complex actions.

Generally the approaches presented seem to work well. Only few partners in the MAP-TN formulated needs for change in the future. Issues were raised about the composition of the selection committee in the Hungarian case. There was also a question of reduction of administrative procedures while doing more explicit communication work (ES, IE) and further improvement to the interface between evaluators and programme management (BE).

Most of the Competence Centre programmes try to come forward with "one product" while cluster-like programmes tend to show a more differentiated funding portfolio. This means a necessity for Competence Centre programmes to stick to one kind of centre structure (but remember the Austrian case with the existence of another network-like centre programme by another funding authority). Within this strict frame (AT, SE, HU, DE, EE, AU, CA), the range for negotiation and differentiation is necessarily broad, but "we did not accept networks between universities" (SE), "the centre must have a physical core and must be a legal entity" (AT).

On the other hand cluster like programmes often have a number of sub-schemes (SL, UK, FR, $\mathrm{NL}$ ) to better meet a specific mission - or subsidiary objective - of the programme and offer less flexibility within these measures. There may be a link with the number and volume of applications too. It seems to be easier to find individual solutions when negotiating with a small number of large consortia. 
Examining the critical success factors, it is also necessary to understand what did not work properly in our MAPs and the risks that have to be managed:

- The extent to which administrators, evaluators, applicants and others are able to fully comprehend the programme goals and processes.

- Ranking based on multiple soft criteria generates uncertainty among administrators and stakeholders with respect to the final choice of awards; investing on the basis of potential future impact is a new and uncertain departure for most. The concern over evaluating applications in terms of their future benefits is a classic challenge of ex ante impact assessment.

- National competitions - thematically open - can lead to a situation where demand for support far outweighs available funds (number of awards), which in turn causes substantial wasted effort and many disheartened researchers outside the lucky few centres.

- The scale of the projects and their duration means that programme portfolios are scantily populated and offer little opportunity for steering and adjustment of risk profile. 


\section{CONTRACT NEGOTIATION AND FUNDING PRINCIPLES}

\subsection{Introduction}

Satisfactory performance of a project is dependent on a true meeting of minds concerning how the project will be implemented, the terms and conditions applied, and the deliverables expected. For this reason, the contract negotiation phase is of vital importance. The purpose of this phase in developing a successful project is to clarify and agree all aspects of the project, the work programme and the expectations of all parties to the contract ${ }^{23}$. Effective contract negotiations create a climate in which concerns, constraints and requirements can be discussed openly. The overall aim should be to ensure no surprises at a later stage.

In general, the more complex the programme, the more complex the negotiation process. In MAPs, many partners are usually involved in the proposals; the work programme is usually complex, relatively long-term and multi-dimensional in nature; and the funding and contractual arrangements may also be complex. Accurate specification of what is expected in terms of outputs may be difficult. For these reasons, the contract negotiation phase assumes a particular significance.

Because funding is normally a key aspect of contract negotiations, the principles by which MAPs operate the funding of projects are covered in this chapter also. The chapter covers:

- The composition of consortia within MAPs;

- Costs and funding issues;

- How contract negotiations are organised and carried out;

- How Intellectual Property Rights (IPR) are dealt with in contracts;

- Requirements in relation to publication and reporting of results;

- Arrangements for auditing and verification.

It will be seen that there is considerable variation between MAPs in the approaches taken to all of the above issues. There are often good reasons for these variations, arising from particular conditions within which different MAPs are implemented. Thus, although we aim to produce a set of good practices, it also must be noted that actual best practice within a given MAP is contingent upon many external factors.

${ }^{23}$ Of course, the rules and requirements concerning issues such as consortium composition, IPR, etc, should be made clear to potential applicants at a much earlier stage - prior to submission of proposals. No key requirement of the programme should come as a surprise after they have been approved for the MAP. However, there are often many details that remain to be agreed finally at the contract negotiation stage. 


\subsection{Evaluator input to the contract negotiations}

In all MAPs, the agreed work programme forms the primary specification for what will be done, what resources will be involved, and what outputs will be delivered. In most cases, the proposal evaluation stage will throw up suggested amendments, and these represent the main basis for negotiation. It is necessary to ensure that the work programme that forms part of the contract takes account of the suggestions from the evaluation.

Sometimes these will be agreed during the negotiation, and there is then a need to modify the work programme annexed to the contract. In other cases, the proposers are first invited to consider and take account of evaluator comments, and to modify the work programme accordingly, prior to the negotiation stage. Modification of the work programme prior to negotiation would seem to represent the most rational approach, since this may reduce the number of issues to be discussed in the negotiations themselves.

\section{Exhibit $14 \quad$ Key points: Evaluator input}

\section{Evaluator input}

A strong proposal evaluation, with constructive suggestions for improvement, provides an excellent agenda for the contract negotiation process

The more independent and expert the external evaluation, the more likely that the proposers will see the benefits of modifying the proposal.

Proposal evaluation provides an opportunity for a strong international input to the process, where this is felt to be desirable.

It is highly desirable that proposers be required to consider and take account of evaluator suggestions, prior to and during the contract negotiations.

\section{3}

\subsubsection{Composition of consortia}

By definition, all MAPs involve consortia of some kind, involving partners from a wide range of institutions and sectors. The programmes represented in MAP-TN are no exception to this: all of them involve consortia, and these are usually a considerable number of partners composed of organisations from the scientific community (higher education research and research institutions) and from industry. Typically, there are more industrial partners than academic, although the precise combination is dependent on the aims of the MAP.

24 Once again, the MAP requirements with regard to consortia should be made clear long before the contract negotiation stage. However, it is the case that there are frequently outstanding issues and requirements to be discussed and agreed during negotiations. 
However, the composition of consortia is dependent on the programme itself, and varies greatly from MAP to MAP. In one case (UK), 95\% of the programme funding is awarded to consortia, with the other $5 \%$ being composed of small awards to single companies. In another (BE), some two thirds of proposals involve consortia of research groups across institutional boundaries. In DE programme Nanotechnology CC, applicants are single institutions, but individual CCNs are themselves composed of more than one organisation, either organised as a registered association (with membership fees) or as loose networks in which the partners sign declarations of intent to contribute to the network. Sweden's Competence Centres (CC) Programme usually involves multiple departments from a single university, together with from six to more than 20 industrial partners. The other CC programmes from AT, $\mathrm{HU}$ and $\mathrm{EE}$ and require similar consortia. The CA NCE programme requires including the "receptor community".

\subsubsection{Required organisational form for consortia}

As with the composition of consortia, the organisational requirements vary from MAP to MAP depending both on the nature of the programme and on the legal and procedural requirements pertaining in the country. Indeed, the variations in requirements are very broad.

\subsubsection{Organisational structure}

There are no specific requirements for the legal structure of consortia in the preparatory stage e.g. in AT, ES, UK, HU, SL or IE, although in some of these cases the practice is to have some form of contractual arrangement between partners to regulate the operation of the contract. In the $\mathrm{K}$ plus programme (AT), for example, while there is no requirement for a legal form of the consortium, the inner structure of the Competence Centre being proposed must be clearly structured in the proposal, and Letters of Intent among the partners are considered to be of importance. For the operation of the Centres, it is required that they form a company with limited liability $(\mathrm{GmbH})$. In $\mathrm{AU}$, as for example, Centres can be either incorporated companies or unincorporated joint ventures. The majority of CRCs is currently operated as unincorporated joint ventures but incorporation would be preferred by the CRC programme.

In many cases, there is a specific requirement that the proposal involves primarily organisations based in the host country. However, the rules for this vary in strictness from country to country: in SL, the requirement is that the consortium must be an organisation registered in Slovenia, but in practice this may mean that the main contractor is Slovenian. EE has stricter requirements: at least one of the scientific partners and three of the industrial partners must be registered in Estonia, even though other partners may be non-Estonian. The whole consortium must be at least $51 \%$ Estonian.

\subsubsection{Changes to the consortium}

Inevitably, in a contract of long duration the need to alter the composition of a consortium will arise from time to time. This may be because one or more partners fail or are unable to deliver what has been agreed, or become less relevant to the work of the project. In some cases a partner may face financial problems. It may also be because an unforeseen need arises to augment the team. 


\subsubsection{Key principles for consortium organisation}

Whatever the composition or form of the consortium, a number of central principles emerge. These include the following:

It is important to ensure that one organisation acts as lead partner throughout, including negotiation of the contract and contractual responsibility for implementation and reporting. Some form of agreement between partners is important - at the very least to ensure that all partners are aware of their obligations in performance of the contract, and to ensure that arrangements for IPR are clear to all consortium members. Although this may be nothing more than a Letter of Intent from each partner at the initial stages, it is highly desirable to ensure that a more detailed contractual arrangement is drawn up between the lead partner and the others in the consortium. Such an arrangement should specify what is required both in terms of the work to be done and in terms of the deliverables and reporting requirements.

Contractual arrangements between partners should not be more complex or detailed than the project requires. However, they should be adequate in relation to the project and the contract; the more complex and/or long-term the contract, the more detailed the arrangements should be.

Requirements for the national identity of a consortium should be spelt out clearly. Bearing in mind the direction of European RTDI programmes, consideration should be given where possible to the opening of programmes and consortia to organisations from other countries.

\section{Box 20}

\section{Organisational form required in Sweden's Competence Centre (CC) Programme:}

In Sweden's CC Programme, each Competence Centre is hosted within a single university. However, since the Centres perform multidisciplinary research, research groups from several departments within the university are usually involved. All Centres must have a number of actively participating Industrial Partners, numbering between 6 and more than 20 companies.

Because the Centres are organised within a university, they are not required to form a legal entity. Most but not all Centres are linked to a host department, and in some cases they report to the office of the university President. However, the CC Programme requires a three-year Principal Agreement to be in place, and this specifies the internal organisation of the Centres. Each Centre is managed by a Board of Directors appointed by the university in consultation with VINNOVA (the MAP manager) and the Industrial Partners. A Centre Director is appointed by the university, and is responsible for the operational management of the Centre's activities. Collaboration Agreements are signed between the host university and other research institutions involved. An Incorporeal Rights Agreement is also signed with the Industrial Partners; this regulates such things as property rights, transfer of project results, publication, etc. Ten success criteria for the operation of Competence Centres are appended to the Principal Agreement.

Thus, although the programme does not require any specific legal entity to be established, it does ensure that a comprehensive set of agreements between all partners is in place; it specifies the internal organisational requirements for a successful consortium; and it specifies the main criteria for satisfactory performance. 
Exhibits 15 and 16 illustrate two similar organisational / contractual links between the funding agencies involved, the host universities/higher education institutes (HEls), the Ministries involved, and the other partners.

\section{Exhibit 15: Organisational and contractual links: Hungary’s KKK Programme}

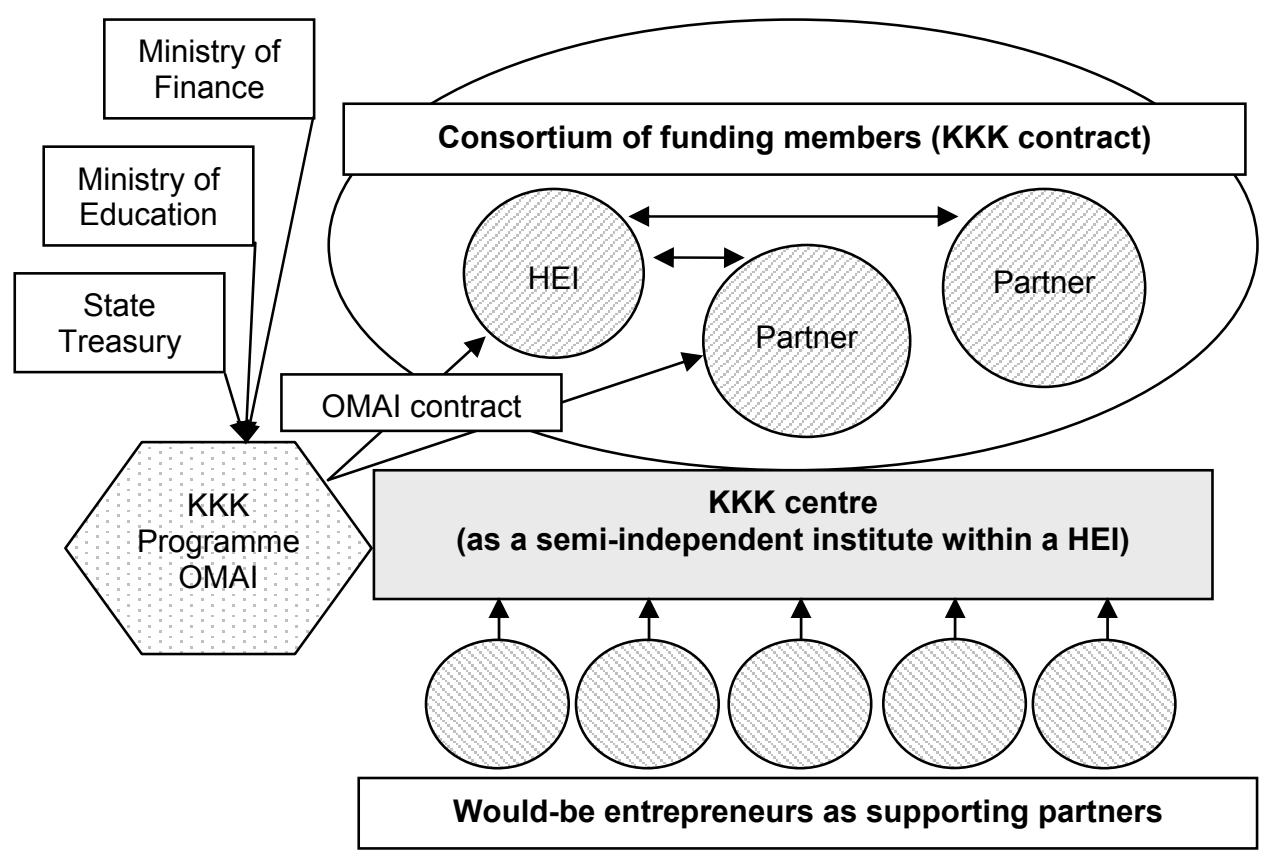

Notes: The main contracts in the KKK programme are: (i) The OMAI contract(s), which represents the main contract between the Ministry/MAP Managing Agency and the HEI host; (ii) The KKK contract, which represents the contract between the organisations hosting and supporting the KKK (Competence) Centre

Exhibit 16: Organisational and contractual links: Sweden's Competence Centre Programme

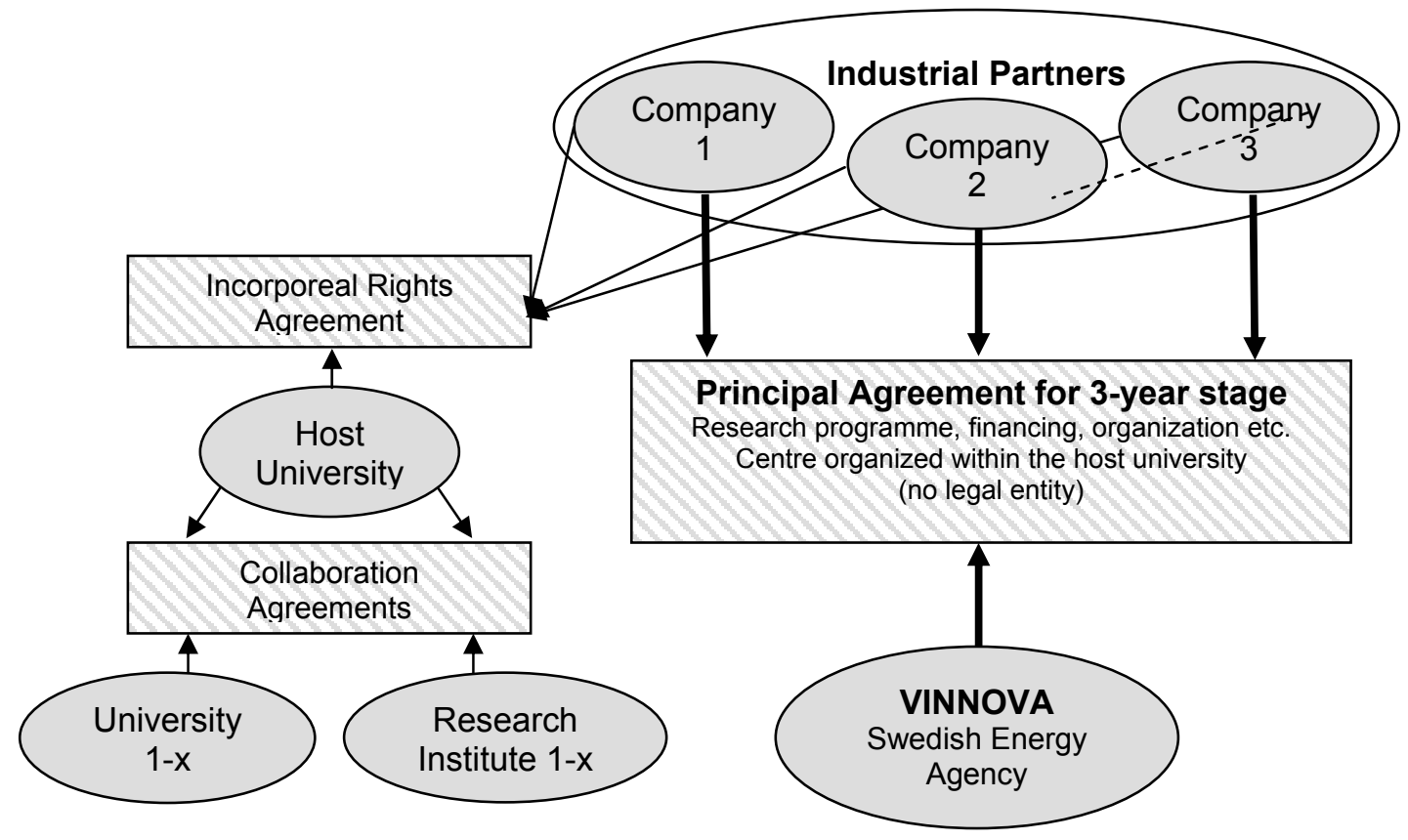




\section{Notes:}

- Principal Agreement - is an agreement between the parties cooperating in a Competence Centre - a number of Industrial Partners, a university, and the MAP management agencies, VINNOVA or the Swedish Energy Agency, STEM. The agreement is signed and entered into for each stage. It regulates the performance and joint financing of the research cooperation, the rights to use results, reporting, evaluation, audit, confidentiality etc. The agreement includes a long-term strategic plan, a research programme and a budget for the stage in question.

- Incorporeal Rights Agreement - is an agreement between the university and all the Industrial Partners, which regulates (among other things) the rights to acquire project results, payments to creator at the university for grants and transfer of project results, publication, etc.

- Collaboration Agreement - is an agreement that regulates research work from other research providers such as other universities, industrial research institutes, other organisations or individual people whose participation within the Competence Centre is stated in the operating plan but which is not a party to the Principal Agreement.

- Connecting Agreement - is an agreement connecting an Industrial Partner as a party during the present stage.

\subsection{Funding and costing principles}

If shared goals represent the main reason for developing a consortium to submit a proposal in the first place, funding is what binds the partnership and facilitates the collaboration. There would be no proposals without funding. And the level of funding determines the level of influence of all parties to the contract, in shaping the work programme and in determining who has legitimate rights in relation to what is done and how. Thus, funding is not simply about providing the necessary resources, but is also a principal determinant of influence among the parties. Most specifically, the level of funding determines to what extent the programme itself can dictate what is and is not to be done within the project. A low level of funding, then, may allow for a wider spread of contracts, but it also reduces the influence of the programme itself. This may be an issue if the programme has over-arching aims that transcend the aims of any one project (for example, one of the policy aims of the European Union FP6 is to advance the European Research Area).

It is also necessary to ensure that the arrangements for funding and the costing of work are equitable, and that the rules for payment reflect the principle that only those costs allocable to the work of the project should be paid. 


\subsubsection{Programme funding levels}

For the partners within MAP-TN, there are quite considerable variations in levels of funding offered. For example within the PII programmes (UK), the funding level is nominally $50 \%$ of project costs, but in practice the level depends on the degree of market failure being addressed, and the actual funding levels vary. Also, the level of funding has dropped over time, with $52 \%$ being the average level in 1997, but $42 \%$ being the average for 2002 ; the main reason for this has been a desire to support a greater number of initiatives. Usually the level of funding lies within the range of $40 \%$ to $60 \%$, with higher levels of funding for some projects.

A number of underlying principles emerge from an assessment of MAP-TN programmes:

- Funding is lower for private sector organisations (industry) than for public sector organisations such as research institutions. Typically, industrial participants receive from $30 \%$ to $60 \%$ funding, while public sector/higher education institutions are eligible to receive up to $100 \%$.

- Funding is higher for SMEs than for larger enterprises - for example, ES provides up to $50 \%$ funding for SMEs and up to $40 \%$ for larger enterprises.

- Funding tends to be higher for: long term, basic/strategic research; and for public good projects (for example, IE), which often receive up to $100 \%$ funding.

- Funding tends to be lower for: demonstration projects; applied research that is close to the market; and in projects where direct partner exploitation of the results is likely.

Note that funding quota are often depending on the rules of EU competition policy and it's R\&D state aids framework. 
Box 21

\section{Policy objectives in state funding: - Estonia's Competence Centre Programme}

In Estonia's Competence Centre Programme, the aim is to achieve an appropriate mix between basic, industrial and pre-competitive research, although in the longer term the aim is for Competence Centres to concentrate on industrial research. In accordance with the legislation on state aids, the programme can provide up to $100 \%$ state funding for basic research, up to $50 \%$ for industrial research, and up to $25 \%$ of costs for pre-competitive development. Higher levels of funding may be available for SME involvement, location of the R\&D operations, and international cooperation.

Box 22

\section{Policy objectives in state funding:- The UK PII Scheme}

Over the past six years, the UK Ministry has been anxious to increase the financial gearing within its PII Scheme. Applicants compete on 'Aid Intensity' and, as a result, government funding has reduced from $52 \%$ in 1997 to around $42 \%$ in $2001 / 2002$. This is partly because of the high level of demand for funding through the PII programmes, and partly in order to award different levels of support depending on the perceived degree of market failure.

\section{Box 23}

\section{Policy objectives in state funding:- Hungary's KKK Programme}

As in Estonia, the Hungarian KKK programme supports the establishment of Competence Centres. State grants provide funding for all operating costs other than acquisition or renting of buildings, or payment of taxes. The longer term aim is to reduce dependence on funding, and for that reason the maximum funding levels provided are $50 \%, 40 \%$ and $30 \%$ of costs in the first, second and third three-year funding cycle, respectively.

Funding levels are guided by at least four policy objectives, and it is necessary in the design of a MAP to identify which of these are of the greatest importance:

- To provide encouragement for long term research that is less likely to attract private sector funding, and that is considered to be research for the public good rather than for short term returns. The corollary to this, of course, is that less funding is provided for RTDI that is close to the market.

- To maximise the gearing provided by state funding. This is a policy objective pursued when the total state budget is limited compared with the demand for funds from the marketplace.

- To reduce dependence on state funding over time. This seems appropriate where an organisation is being established that is expected to become self-funding in the longer term.

- To meet other policy objectives, such as encouraging SME involvement, providing incentives for the development in selected geographic locations or technology fields, or encouraging international cooperation. 


\subsubsection{Matching funds, cash and in-kind contributions}

All of the MAPs represented in MAP-TN require matching funding in some form, with the exception of some specific programme elements (e.g. public good studies/projects - IE - and some programme elements that provide $100 \%$ funding for basic/pre-competitive research - e.g. EE, BE). The MAPs in AT, ES, EE, HU, IE, SL and UK all allow in-kind funding, though some (AT, EE) place a maximum of $50 \%$ in-kind provision of matching funds, and $\mathrm{HU}$ has a limit of $10 \%$ in-kind funding, which can be composed of laboratories, equipment and other assets. For $\mathrm{SE}$, in-kind contribution from industry is both desired and expected and may be from $35 \%$ to $100 \%$ of the matching funds. The StarMAP examples AU, CA and NO allow in-kind funding of up to $50 \%$, for the other StarMAP countries there is no information.

Where in-kind funding is permitted, it is usual to have a requirement that a reasonable financial value be attributed to it. In the PII scheme (UK), the in-kind funding must also be defined in terms of the added value to the project, in order to ensure that it is valid as matching funding.

\subsubsection{Some key principles of MAP funding}

Drawing from the experience of the MAP-TN partners, the following principles are suggested for funding of MAPs:

- Some form of matching funding from the partners is normal and is an expression of their interest and commitment to the project.

- Funding levels often vary, and this is generally considered to be an important way of recognising either the relative importance of the outputs to the funding agency or the nature of the research being undertaken. In general, public good and long-term, basic or precompetitive research receive up to $100 \%$ funding; funding to industrial partners varies from $40 \%$ to $60 \%$, and SMEs generally receive higher levels of support.

- In-kind funding by partners is usually permitted up to a set limit of, say, $50 \%$ of the matching funds.

- It is desirable to have some form of assessment of in-kind funding, to establish its real financial value and its likely relevance and contribution to the work being undertaken.

\subsection{Contract negotiations}

As indicated above, contract negotiations represent the stage in the process at which real agreement can be reached between the contracting parties. For this reason, it is usually a vital element in the process.

AT, DE, EE, HU, IE, SE, SL, NL, FR, FI and AU all have a contract negotiation stage in their procedures; ES and BE in general do not, and the proposals in these cases are usually either accepted as they are or are modified by the proposers in accordance with recommendations from the proposal evaluators. The exception is NO (which is no MAP according to our definition) where the Technology Attaché takes care about all administrative issues to facilitate the settingup of projects. 
Contract negotiations within MAPs are often complex and multi-dimensional, reflecting the complex nature of the programme itself and of the projects adopted by it. The fact that many projects are long-term and strategic in nature also tends to make for longer and more complicated negotiations. Other determining factors may include: lack of prior experience in collaboration, differences in motivation among parties, and unbalanced bargaining power. Of course, the application procedure (one- or two-stage process, for example) also has a major bearing on the length of the negotiations.

\subsubsection{The negotiating parties}

Where MAPs are generally being administered fully by a (programme management) agency on behalf of the government, it is usual for the programme negotiating team to be drawn from that agency. This would appear to be the case especially where the agency is a public sector one managed by the funding Ministry. However, where the agency is very close to its parent Ministry, it happens that the negotiation team is to be made up of staff drawn from the agency and from the Ministry, although the agency normally manages and oversees the process. In other cases the Ministry commonly acts in a support or advisory/approval role. A desirable role for the parent Ministry(ies) is as a body that can be referred to for a negotiating mandate in relation to sensitive proposals, or for proposals that are marginal in terms of their conformity to the MAP conditions, but desirable from the point of view of meeting strategic objectives. In such cases, the Ministry can either confirm the agency's negotiating mandate, or serve as an external reference party whose function is to ensure adherence to the conditions of the MAP.

The negotiators on behalf of the proposers always include the lead partner, at a minimum; it is important to have the lead partners involved, as it is they who must take responsibility for the outcomes. In six cases within MAP-TN (DE, EE, BE, IE, SL and UK - PII Open/Focus programme elements), only the lead partners are involved in the negotiations. In all other cases where negotiations are involved, the negotiating team for the proposers includes some or all of the partners. It would seem that the more complex, long-term and strategic the proposal, the more relevant it is to involve all of the partners in the negotiation process. The disadvantage of this is that it means a much greater number of negotiators, and in consequence the risk of a drawn-out process. Its advantages are that all partners are fully involved and are directly aware of the issues and outcomes being discussed; and that the partners gain a direct understanding of the priorities and concerns of the programme management.

Based on the experiences of the MAP-TN partners and StarMAP, the following generalisations seem to apply: (i) Where the proposal is for long term, basic research, and has a strong academic focus, the negotiation tends to be with the whole consortium; (ii) Where the proposal is for applied research, negotiation tends to be with the lead proposer; (iii) Where the proposal relates to establishment of an organisation (e.g. a Competence Centre), negotiation tends to include the host organisation, the director of the new organisation, and at least a sample of the academic and industrial partners within the proposal. 


\subsubsection{The method of negotiation}

Most contract negotiations involve face-to-face negotiation meetings - most usually a series of meetings held over a period of one to four months. Elements of MAPs that are more straightforward, involve shorter and less expensive contracts, and/or intended to provide a 'fasttrack' option for smaller projects with limited objectives, may be negotiated without a negotiation meeting. In such cases, if changes are required to the original proposal, these may be conveyed by letter, e-mail or through telephone conversations.

\subsubsection{The negotiations themselves}

Because the contract negotiation process must be contingent upon the nature of the MAP and the specific proposal being discussed, there is clearly a wide variation between MAPs in terms of the way negotiations are organised. However, based on the MAP-TN experiences, a number of principles for an effective contract negotiation emerge:

- A strong proposal evaluation provides a good basis for an effective negotiation, particularly if all parties have been well briefed on the conclusions of the evaluation, and the issues and concerns that have emerged. The evaluation stage also offers an opportunity for a strong international input to the proposals. An international input may be viewed as an impartial assessment of the issues, and this may be useful in providing a set of views that will be acceptable to both negotiating parties.

- Evaluator comments and suggestions should be taken into account in preparing the negotiation agenda. While this may seem obvious, some MAPs use other means of dealing with evaluators' comments.

- All experienced negotiators agree on the importance of a clear set of objectives for the outcome. The well-known categorisation ('M-I-L')of negotiation objectives into 'Must' (those outcomes that you must achieve in order to be able to reach agreement), 'Intend' (those outcomes you believe are desirable and achievable), and 'Like' (those outcomes that are peripheral but desirable if they can be achieved) provides an excellent preparation framework for contract negotiations.

- The most effective negotiations seem to be organised as a flexible, informal process, not too tightly specified in the MAP work programme. This allows the process to be managed in an organic way, responding to issues specific to each contract negotiation, and flexing as necessary during the process in order to respond to and deal with any issues that arise. A broad overall framework designed to bring some clarity to the expectations of both parties is highly desirable, but this should not exclude the possibility of varying the process as it proceeds.

- Because the negotiation stage can take some time, it is critical to commence the contract negotiation phase as promptly as possible after the proposal evaluation and approval stages have been completed. This also ensures that any concerns or issues raised during the evaluation remain fresh in people's minds. 
- An agreed timetable is important. Deadlines help to ensure progress within a reasonable time period. They also allow the MAP manager to monitor and control the lead-time from approval of the proposal to conclusion of the contract. And deadlines require the partners to take a business-like attitude to resolving the critical issues that usually exist in every complex negotiation, and that often are left until the closing stages.

- It helps to impose a small number of intermediate milestones on the process, in order to ensure reasonable progress through the negotiation agenda.

- International experience on negotiations indicates that skilled and experienced negotiators tend to lead to fairer and better outcomes, even where the balance of power is very unequal between negotiating parties. MAP managers should ensure that their teams have been well trained and well prepared. It may sometimes be desirable to assist proposers to prepare for the negotiation, as this can lead to a better resulting agreement.

- One of the most important traits of effective negotiators is the ability to listen to the other side. Listening skills are more important than verbal or persuasion skills, and a mix of nondirective and directive approaches is much more effective than either a solely directive or non-directive approach.

- Negotiating parties often adopt positions based mainly on the function and roles of their organisations. While this is inevitable, it may be more important to focus on achieving outcomes that will maximise performance and the exploitation of results than to focus on a narrower set of organisational priorities. Focusing on an effective outcome also permits an agenda of discussions that both parties can understand and accept.

- One of the most critical tasks for MAP managers is to create a spirit of collaboration and trust, rather than one of competition and suspicion. Seeking common ground, recognising and supporting the genuine concerns of the other side, and testing and expressing understanding are powerful behavioural tools in developing and maintaining a culture of collaboration. Such apparently 'soft' approaches can often be extremely tough in practice, and they do not have to mean an absence of strong negotiating objectives.

Equally, there are many pitfalls to be avoided in the contract negotiation phase:

- It is easy to be distracted by the inevitably large number of small issues to be discussed, and to forget the small number of key issues. If possible, the important issues should at least be discussed in the early stages, although they may then be deferred until other issues have been agreed. Negotiations should not be allowed to break down or be delayed by relatively unimportant issues.

- Proposers invariably have a high level of commitment to their projects, and often a high level of expertise on the issues surrounding it. This is often translated into commitment to negotiation objectives, and a high level of energy and zeal in the negotiation process. It is important that the MAP management team match this. It is also important to retain sufficient flexibility to recognise the positive aspects of the proposers' viewpoint, where these exist. 
- The parties to the negotiation should be knowledgeable about the scientific and socioeconomic potential of the project, as well as the risks and financial and management aspects. A negotiation between the proposers and a MAP team comprised of people not closely familiar with the topic and the MAP should be avoided.

- It is important to ensure that any additional advisory resources brought into the negotiation or to the implementation are really engaged with the project. Not to do so is to risk adding to resources or delays without any commensurate return to the project.

- Contractual regulations are important but they should not become the overriding topic in negotiations. Of course, critical regulations and procedures must be adhered to, but they rarely represent a truly strategic aspect of a proposal.

- Negotiations should not focus on one major issue to the exclusion of all others. The solution to important but apparently intractable negotiating problems often lies in creative packaging of several elements of the negotiation. The principle that "nothing is agreed till everything is agreed" is often very helpful when attempting to create multi-dimensional outcome packages to which everyone can agree.

- Finally, a negotiating team with power on its side (and MAP managers usually have the ultimate power of accepting or refusing the proposal) can sometimes use this power excessively, to obtain an outcome with which they are satisfied, but with which the other side is extremely dissatisfied. The aim should be to achieve an outcome with which everyone expresses equal satisfaction.

\subsubsection{Duration of the contract negotiation phase}

Among the MAP-TN partners and StarMAP examples, there is a wide variation in the expected duration of the negotiation stage. As expected, negotiations tend to take longer where large, complex proposals with multiple partners are under discussion, and shorter in the case of simpler proposals. Also, the context influences duration: a MAP within a highly proceduralised or bureaucratic setting can take much longer than one where the decisions can be taken at the level of the negotiating team.

The general principle should be that negotiations should take as short a duration as possible, commensurate with a satisfactory outcome being achieved. In general, negotiations take longer (up to 18 months) where: (i) A new organisation is being established as part of the contract; (ii) There are multiple partners; (iii) The proposed work programme is long term in nature.

The average contract negotiation among the MAP-TN partners is about 3 months, and this seems to represent a reasonable time period for most MAPs.

Factors affecting the nature and duration of MAP negotiations include:

- It is perhaps unique to MAPs that the contract negotiation represents an important early step in a long-term mutual learning process between MAP manager and the proposers.

- A high level of discussion and consultation is necessary before reaching a comprehensive and effective agreement. 
- Less standardisation is usually possible within MAPs; thus, a longer negotiation may be expected.

- It is often necessary to consider each step very carefully, and simple procedures or rules may not work in such a setting.

- In MAPs, the most significant outputs may also be the most intangible ones in the contract negotiation.

- Finally, MAPs are relatively new instruments, and MAP managers and proposers alike are still on a learning curve.

\subsubsection{Contract finalisation}

MAP-TN and StarMAP experience suggests that very few negotiation teams have the authority to approve the outcome of the contract negotiations without referring to some other authority. This may be (and most usually is) a matter of referring the negotiation outcome for approval at a senior level within the MAP management agency, or alternatively of referring it for approval by a specific approvals board. In some cases it is necessary to obtain the approval of the funding body (Ministry or parent organisation); however, where the MAP management agency has clear responsibility for implementation of the programme, it is desirable that this should extend to the final decision - with referral to the funding body only being required when legislation demands it, or when the outcome implies a deviation from the established procedures or policies for the MAP.

The most important points here are that:

- The policies and procedures determining what can and cannot be accepted within the MAP should be clearly established and understood by all parties.

- The MAP management agency should have clear responsibility for effective implementation of the programme, and they should assume the authority necessary for that implementation, so that referral upward is by exception rather than being the rule.

\subsection{Contractual issues}

\subsubsection{The contract itself}

Within MAP-TN and StarMAP, all but one (ES) of the MAPs involve the signing of specific contracts. In the case of ES, acceptance by the proposer of the funding implies acceptance of all aspects of the governing decree.

Seven of the MAPs in MAP-TN (AT, DE, UK, HU, SL, BE, IE) and all StarMAP programmes utilise a contractual form that is the 'standard' one for the agency, with either the work programme or a more complete agreement attached to the contract as an annex, with details such as the work programme, key deliverables and outcomes, conditions governing issues such as Intellectual Property Rights (IPR), eligible costs, reporting requirements, conditions for exploitation, and special payment terms or other conditions. 
In the case of $\mathrm{HU}$, the governing contract is a standard 'model' contract which includes references to the decree establishing the MAP; the contract for the Competence Centre, which is an agreement between the founding members, plus an agreement on IPR, are attached as annexes. SE has a more complex and tailored contractual approach, with a Principal Agreement signed between the MAP managing agency, the host university and the industrial partners. There is also a requirement for Collaboration Agreements between the research institutions involved, and an Incorporeal Rights Agreement between the host university and the industrial partners (see case study above in this chapter). A similar contract has to be signed within the other CC programmes, like in AT, AU and CA.

Contracts typically cover a wide range of issues, including payment terms, reporting required, dissemination and exploitation of results, IPR, termination of the contract, changes to the consortium and/or work programme, and success criteria.

\subsubsection{Checking state aid/quota provisions}

$\mathrm{HU}, \mathrm{SL}$ and EE MAPs involve approval of the financial/funding aspects of the contract by the parent Ministry; in $\mathrm{HU}$, the financial aspects are checked by the MAP manager, but in consultation with the Ministry of Education (R\&D Division) in relation to the proposed R\&D activities. And in Australia, the CRC secretariat is part of the ministry carrying out all aspects of the contract negotiation.

AT, DE, ES, UK, SE, BE, IE, NL, FI, FR and CA MAPs allow for checking by the MAP agency itself, although the German MAP procedures also allow for Ministry involvement along with the agency. In the UK and elsewhere, the funding body is involved only on an exception basis. In NO the Technology Attaché has the overall power.

It would seem to represent good practice to place the responsibility for such checking with the MAP management agency, since this represents one aspect of programme management, and since it forces the MAP manager to adhere to accepted funding and quota procedures as part of this management function. However, clearly this must be governed by the prevailing national regulations and policies on such matters.

It should also be noted that in Europe the programmes themselves must normally be checked against EU Directives on state aids (as part of the European competition law, see chapter 8 on governance). This is normally undertaken at Ministry level, but it is usual for this to be done in relation to the entire programme rather than in relation to specific contracts.

\subsubsection{Payment schedules}

There is widespread variation among the MAP-TN partners and in StarMAP examples as to the phasing and timing of payments. In some cases (HU, UK, SL EE, FI IE), stage payments are determined by the timing and size of each milestone in the work programme. In AT and Australia, the contract sum is broken into annual budgets, with quarterly payments based on these budgets. In DE, payments are also made quarterly, and in ES payments are made annually. In BE, the norm is to allow for nine equal instalments spread over the four-year term of the contract. CA and FI pay once a year and the NL twice a year. 
Relatively few MAPs involve initial, up-front payments unless the work programme is such that this is necessary for implementation. Money withheld as a final phase payment is reported for DE, UK, FI, AT and NL, in these cases this final payment is between $5 \%$ and $20 \%$ of the full budget.

Some principles for payment schedules:

- Stage payments are best agreed on the basis of the work programme packages and the budgets required for each package;

- Payment terms should be such that they do not place an undue liquidity burden on the participating organisations;

- Up-front payments may be necessary where there are substantial set-up costs;

- Funds withheld till adoption of final reports can place a heavy cash flow burden on consortia. Where they are regarded as necessary, they should be kept to as small an amount as feasible.

\subsubsection{Reporting}

AT, DE, ES, SE, HU, SL, EE, FI, NO, AU and CA all provide reporting guidelines as part of the contract. UK does not include guidelines in the contract, but standard forms are provided for administrative reports. In the NL the forms can be downloaded from the net.

Provision of a broad framework for mandatory reporting would seem to represent good practice. It clarifies to the consortium what is expected by the MAP manager, and it increases the probability that acceptable reports will be delivered. A clear reporting framework also increases the uniformity of progress reports, which in turn makes for much easier administration by the MAP manager.

\subsubsection{Intellectual Property Rights (IPR)}

IPR represents one of the most difficult aspects of complex RTDI projects, and is a matter of concern both to MAP managers and to consortia. Different MAPs handle the issue differently (see cases). However, the general principles for IPR within MAPs would appear to be as follows:

- The contract should set out the principles for IPR very clearly - this is often not the case, but there are very good reasons for adhering to this principle;

- The originator of the innovation or a consortium partner (or in $\mathrm{CC}$ programmes the centre) owns the results;

- Normally, all partners have (at least) non-exclusive right of use;

- In contracts involving close collaboration between partners, it is desirable to ensure that any contract between them includes a specification of the rules for handling IPR and related issues;

- The MAP management agency or Ministry retains the right of publication; 
- It may be appropriate for the MAP agency to offer assistance in managing IPR;

- In some MAPs, the contract stipulates that IPR must be applied for promptly. This is a worthwhile idea, since obtaining IPR is a vital requirement for exploitation and commercialisation.

Overall, the aim should be to strike a sensible balance between the need to protect IPR and the need and desire to disseminate the results.

\subsubsection{Publication of results}

In most MAPs, publication of results is considered desirable at least, and is often mandatory. Indeed, the number and quality of publications arising from the contract represents one of the dimensions for evaluation of the contract.

It is normal for MAP managers to retain the right of limited publication, but this often extends only to publication of bare details of the project: the title, partners, duration, and a description. However, it can also extend to limited publication of a more detailed description of the project, together with a summary of the results, possible applications, benefits, further work required, etc.

It is also normal to expect the contractor(s) to publish more detailed results in suitable journals refereed wherever possible. The originator of articles and papers may be the organisation(s) centrally involved in obtaining the results, or the lead contractor, or all of the partners. In some cases, the contract stipulates that all partners must agree with the content of any proposed publication, and this would seem reasonable.

In other cases, the MAP agency works with the contractor to develop an effective exploitation plan. Where this is appropriate, it is a desirable activity for the MAP agency to engage in, considering the importance of effective dissemination.

\subsubsection{Amendments to the contract}

Ten of the MAP-TN MAP-TN programmes permit at least some amendments to the contract (AT, DE, ES, FI, UK, SE, HU, EE, BE, IE); one (SL) does not. It is clear that at least some of those allowing amendments limit these to changes in budget, and only a few deal specifically with other changes - such as changes to the consortium. DE does not permit changes in costs.

Where complex and in particular long-term contracts are the norm, it would seem advisable where possible to allow flexibility in amendments. Budgetary flexibility is very desirable, and in some cases within MAP-TN (AT, ES, HU, EE) this flexibility is built in through incorporating changes in annual budget and research plans into the means of managing the programme. It is also often desirable to permit changes to the consortium, although this may be limited in order to ensure stability. It is worth noting that the $6^{\text {th }}$ Framework Programme (with long term contracts being envisaged) is structured such that budgetary and consortium changes are expected and permitted. This is in part a reflection of the objectives of FP6 itself, but is also in part a recognition of the realities of long term RTDI activities. 
However, there should be a good reason for any changes proposed, and they should be justified in writing to the MAP manager before permission is granted. It is good practice to have a procedure for major changes that require the MAP manager to have them approved by a higher authority, in the same way that proposals themselves have to pass through an approval process.

\subsection{Concluding note}

Contract negotiations are the stage at which a true meeting of minds and a merging of objectives is achieved between the MAP agency and the proposers. For this reason, it is vital that the procedures for negotiations be thought through very carefully, and well documented both for the MAP managers and for the proposers.

The negotiation phase is frequently the point at which major delays are introduced into the proposal evaluation/approval/contracting phase of a project. It is important to allow sufficient time for effective and comprehensive negotiations to be conducted. However: (i) Contract negotiations should be commenced as early as possible after the conclusion of the proposal evaluation stage; (ii) MAP work programmes should specify a maximum time for negotiations; (iii) MAP managers should monitor the length of time negotiations take in practice, and analyse the reasons for delays when these occur.

The contract itself is where the conditions, procedures, and outcomes of the negotiations are reflected - whether in the body of the contract or in annexes, including the project's work programme and budget. It is desirable to make sure that the contract covers all aspects of the requirements of the MAP. However, a balance must be struck between: (i) The need to be comprehensive versus the need for a contract that is not overly complex or lengthy; (ii) The need for a stable agreement versus the need in practice for flexibility.

Contracts should reflect the above tensions. The result should be detailed but workable.

\subsection{Lessons from the MAP-TN and StarMAP}

\subsubsection{Consortia}

The most appropriate form of the consortium is best determined by the aims of the MAP. However:

- It is vital to ensure that there is one lead partner responsible for all aspects of the contract negotiation and of the subsequent implementation of the contract.

- It is highly desirable to ensure that there is a clear set of arrangements, confirmed by some form of sub-contract or letters of intent, to ensure that all partners are legally bound to the contract.

- Even though there may not be a specified organisational form for consortia, it is important to ensure that the consortium's internal structure and procedures are clearly specified and are adequate to the requirements of what is usually a complex and long-term project. 
- For projects of long duration, it is advisable to have procedures that enable a change in the composition of consortia. Generally speaking, these procedures should allow for considerable flexibility, though not so much flexibility that the stability of the consortium may be threatened, a balance between flexibility and stability should be the aim.

\subsubsection{The negotiation setting}

- Complex and/or long-term proposals usually benefit from face-to-face negotiations involving all of the principal parties. These are usually augmented by written communications or telephone discussions.

- The negotiation team for the MAP should include the organisation charged with management and implementation of the MAP. Where major expenditure or policy issues are at stake, it may be desirable to include representatives from the funding Ministry or body. However, a more effective role for such bodies may often be to act as advisor in sensitive or marginal cases, or to serve as a mandating body external to the negotiations themselves. The need to refer to an external body for a negotiating mandate can be useful at times, although in general the negotiation should include those who are empowered to arrive at an agreed outcome.

- The negotiation team for the proposers should always include the lead partner, for obvious reasons. It is usually also desirable to include any partners who may be considered critical to the success of the consortium, whether because of the level of resources to be committed by them, or because of the level of expertise or credibility they can offer, or because they occupy a strategically important role within the consortium.

\subsubsection{The negotiation process}

- Utilise recommendations and suggestions from the proposal evaluation process in forming an agenda for the negotiations.

- Start the contract negotiations as soon as practicable after the evaluation phase.

- Set a firm deadline for conclusion of the negotiations, but be prepared to extend the period if this is essential to achieving an outcome.

- Establish a sound set of prioritised negotiating objectives, and stick to these.

- Ensure that both negotiating parties are as well trained and prepared as possible.

- The negotiating team for the MAP should have a clear mandate from their organisation(s), and be authorised to reach an outcome, even though this may require formal approval subsequently.

- Negotiations should be seen as a mutual learning process, from which all sides (and especially the work programme) can benefit. This suggests that the MAP negotiators should be prepared to listen and change their views, while at the same time bearing in mind what outcomes are considered essential to achieving the objectives of the MAP in question. 


\subsubsection{Duration of negotiations}

- Negotiations should not be so truncated that the outcome is less than optimal. However, they should start as promptly as possible (see above), and they should be timetabled so as to ensure that reasonable progress is made at all stages. MAP-TN partners believe that a negotiation period of not more than three months should be adequate for most situations. Note that it is very difficult in this stage for the MAP managing agency to get out of the commitment again, so there is not much to apart from threatening to leave from the negotiation table.

\subsubsection{Contract finalisation and approval}

- There should be a clearly laid-down set of policies and procedures that determine where the final approval of negotiation outcomes rests.

- It is usually desirable to ensure that the MAP management agency should have the responsibility and the authority necessary to enable decisions to be taken by that agency. In that case, it should only be necessary to refer upwards where the negotiation outcomes deviate from accepted procedures.

\subsubsection{State aid and quota provisions}

- European state aid provisions are normally checked by the funding Ministry (or appropriate Department) in relation to the entire programme.

- Where national policies allow, checking of financial provisions, quotas, etc, should be a responsibility of the MAP management agency, with exceptions only being referred upward for decision.

\subsubsection{Payment schedules}

- Initial and withheld final payments are usually best held at small amounts, unless the specific work programme requires larger amounts.

- Good practice would suggest that timing and amounts of payments should be linked to budgets compiled from the detailed work programme, and that payment should be linked to concrete deliverables.

\subsubsection{Reporting guidelines}

- Good practice would dictate that contracts should refer to guidelines on the frequency, structure and required content of reports on the implementation of the contract. These should not be too prescriptive, but they should be sufficient to allow the consortium to know what must be reported, and where possible they should introduce a reasonable uniformity in reporting format. 


\subsubsection{Intellectual Property Rights (IPR) and Publication of results}

- IPR is a difficult issue for MAPs, and the response is sometimes to avoid it. However, it is good practice to specify clearly in the main contract how IPR is to be handled. It is also good practice to ensure that partners contract in writing between themselves, with a clause spelling out what has been agreed regarding IPR and the use of results by each partner. It would be the norm that the originator owns the results, but the main issue to be dealt with in contracting is the rights of the other partners (and often the commonly financed centre as well as sometimes the MAP itself). MAP managers should consider the possibilities of offering support to contractors in obtaining IPR, and where feasible it may be wise to stipulate in the main contract that IPR protection should be sought promptly.

- It is good practice for the MAP agency to stipulate what means of exploitation/ publication is expected, in the form of an exploitation plan included as an element in the work programme.

- Where partners are working closely on the research, the content of any publication should be agreed by all of the relevant partners.

- Because publication of results is so crucial to exploitation of results, the MAP agency might consider the possibility of working with contractors to develop the most effective exploitation and dissemination plans.

\subsubsection{0 $\quad$ Amendments}

- MAP managers should assess very carefully the level of flexibility they think should be permitted within their programmes. Bearing in mind the long term nature of RTDI projects, and the uncertainties surrounding their progress, good practice would dictate that MAPs should permit maximum flexibility, provided this is consistent with both stability of the consortium and the aims of the programme.

- It is recommended that procedures for amending budgets, work plans and the composition of consortia should be specified within the contract. MAP agencies should adopt a procedure for dealing with changes; minor changes could be authorised by the MAP manager, while major changes in budgets or the consortium should require an authorisation procedure involving at least some of those involved in approving proposals in the first place. 


\section{EVALUATION AND MONITORING}

\subsection{Introduction}

In the world of RTDI policy, we are still working through the implications of the comparatively new theoretical perspectives, which place change at the centre of economic development and growth and which conceive of the economy in terms of multiple systems rather than focusing on atomistic actors. MAPs are a class of interventions that tackle properties of these systems: Not (only) a narrowly defined research or technological goal should be achieved, but also that an impetus towards a structural and behavioural change in the participating institutions should be provided.

The reason is that classical programme promotion is partly being abandoned in favour of comprehensive attempts to influence whole innovation systems - or sub-systems - by means of policy measures. Especially inter-organisational cooperations are becoming more and more important in order to cope with the challenges of modern innovation systems.

Evaluating MAPs involves tackling more actors and more complex interactions than are normally handled in traditional evaluations of (simpler) interventions, such as single actor R\&D programmes. Using multiple parallel methods remains a hot topic, becoming even more important, but some of the units of analysis change - especially from project level to refocus on institutions, organisation and their interactions.

As the evaluation of MAPs is a rather novel endeavour and as the requirement for the design of evaluations for each MAP is very specific and unique, this chapter intends to give a conceptual overview on the specialities in monitoring and evaluating MAPs in order to highlight the special MAP-challenges and new venues. Apart from the usual handling of topics in the MAP network the conceptual input had a high priority in the discussion, rising up the problems we face. In a next step we collected, summarised and discussed the experiences of the network members and evaluation experts. Thus, a proper combination of "learning from each other" in the network and "learning from experts' inputs" was chosen to meet the demand of a very complex and difficult topic.

As a result, this chapter on monitoring and evaluation is structured in the following way: The first sub-chapter provides some basic definitions and basic question that were addressed. The second sub-chapter provides a comprehensive overview of evaluation approaches. In the third sub-chapter we offer concrete experiences of the MAP-TN members and from the six quite mature StarMAP programmes. Finally, lessons from the MAP-TN and StarMAP are given. Note that StarMAP was especially important regarding evaluation, because most of the MAP-TN programmes are rather young and therefore not always long-standing MAP-evaluation routines have evolved yet. Note also the discussion of ex ante evaluation procedures in other chapters of the roadmap: design process standards for ex ante programme evaluation, selection procedures for ex ante evaluation. 


\subsection{Basic definitions and questions}

\subsubsection{Definition of Evaluation}

Programmes need to show their effectiveness to their stakeholders boards, clients, and funders, to name the most important ones. An evaluation is the way to validate and judge the work of a programme or the included projects, the activities, the volume, the impact, the cost, and the outcomes. It involves the systematic collection of information about activities, characteristics and outcomes. It provides information for policy makers, managers, projects and the interested general public on the suitability of specific initiatives for achieving the intended objectives. It should improve the effectiveness and decision-making with regard to the projects or programmes. Taking this as a basis understanding of evaluation, the following definitions for monitoring, project evaluation and programme evaluation were used:

The term "Monitoring" refers to the periodical collection of data (and indicators) concerning the performance of projects including the financial controlling of the projects.

"Project Evaluation" is defined as a judgement about the quality (performance, scientific quality, impact etc.) of projects, networks or centres. Project Evaluation could be ex ante (selection of projects $=$ appraisal) accompanying, after certain years (interim), after the end of the project (ex post) and in most cases it is carried out by external experts.

"Programme Evaluation" is defined as a judgement about the quality (performance, scientific quality, impact etc.) of a whole programme. Programme evaluation could be ex ante, accompanying, after certain years (interim), after the end of the programme (ex post or backward look) and in most cases it is carried out by external experts.

Moreover, in evaluating MAPs one needs to consider different levels of analysis. For each specific MAP the various levels take different shapes, but in principle three levels can be differentiated, and must be regarded differently in the evaluation design: The level of the whole programme (that itself is embedded into a policy context whose assumptions need to be looked at as well), the level of "centres" or "networks" (if existing; and see above: we talk about a centre as one distinct intervention, i.e. a project) and the level of single research projects.

In order to cope with the complexity of MAPs, one can simply list the most important characteristics (see also chapter 2) that have a repercussion on the evaluation.

\section{Characteristics of MAPs}

- Multi-measures

- Co-operating multi-actorMulti Actors from different worlds

- Addressing whole (sub) systems of innovation

- Complex management and communication structures

- Specific learning elements and feedback loops

These characteristics influence the design and implementation of the evaluation in many ways and on different levels (see also the "Minimum MAP requirements" in chapter 8 on governance). 


\section{Consequences for the Evaluation}

- Proper selection of projects or centres

- New formative elements and the dimension of learning

- The implementation of a proper monitoring system

- The outcome and impact of funding: Choosing right issues and proper measure-mix

- "Systems of evaluation" or "evaluation integrated in the policy cycle"

Exhibit 16: Relevant project level for monitoring and evaluation

\begin{tabular}{|c|c|c|}
\hline Programme level & Centre Programmes & $\begin{array}{c}\text { Cluster and Network } \\
\text { oriented Programmes }\end{array}$ \\
\hline $\begin{array}{c}\text { Relevant project level for } \\
\text { monitoring and evaluation }\end{array}$ & Centres & Projects \\
\hline & $\begin{array}{c}\text { Individual research projects } \\
\text { within the centre }\end{array}$ & \\
\cline { 2 - 3 } & &
\end{tabular}

After having discussed conceptually the challenges of evaluating and monitoring MAPS we will, at the end of this chapter, map the MAP characteristics and the consequences for evaluation.

\subsection{Evaluation Approaches}

\subsubsection{Monitoring of MAPs: needs and pitfalls $\mathrm{s}^{25}$ :}

MAPs need adequate monitoring systems. In contrast to a formal controlling, which looks after the sound spending of money in compliance with the proposal and the guidelines of the programmes etc., a monitoring system is a comprehensive instrument to assist the (self)governance of the programme and its „projects“26. At the same time it serves as a permanent source of information for the funding ministry and / or the programme management. Thus, a monitoring system must take the explicit objectives of the programmes as a starting point and try to mirror not also the input and output dimension, but also the behaviouristic dimension of MAPs.

Ideally the monitoring system integrates the requirements on three levels: funding ministry, programme agency/evaluators, funded institutions/ structures (e.g. centres). The funding political body should get current data on the activities, structures and budget flows of the funded projects. The data needs to be collected on the level of projects (centres etc.) but should be

\footnotetext{
${ }^{25}$ These considerations have been developed in the context of a recent assessment of the Austian $\mathrm{K}$ plus and Kind programmes commissioned by the Austrian federal ministry for transport, innovation and technology and the Austrian federal ministry of economics and labour in 2003 (Edler / Lo et. al. 2003).

${ }^{26}$ Projects are not meant to be individual research projects but rather the new strucutres funded within which single research projects are conducted.
} 
aggregated currently. Monitoring should be designed in order to enable the analysis along different dimensions of importance for the funding body, such as the allocation to technological areas, regions, types of actors etc. The main objective of monitoring here is to enable informed, current and differentiated political communication about the MAP.

A monitoring system should, moreover, deliver data for an accompanying, periodic or ex post evaluation of the projects/centres and the programmes as such. ${ }^{27}$ This improves the adequacy, effectiveness and efficiency of the data gathering of the evaluation. The monitoring data are at the same time important for the programme management/agency for the optimisation of the programme management and accompanying service / coaching activities.

Last but not least, the monitoring should be designed in order to meet the operative and, above all, strategic requirements of the new structures (centres, networks etc.). Monitoring should be understood as an instrument for the "self-governance" ${ }^{28}$. In this context the self-reflection initiated by the monitoring activities is a pre-condition for strategic development. Thus, the collection and presentation of the data should be in the strategic self-interest of the funded structures.

There are a set of pitfalls and potential drawbacks connected with a comprehensive monitoring system as outlined above:

If political decision makers and funding institutions of the programme receive structural data of the programme and its projects continuously, the clear division between the decision-making function and the governance/management of the programme may become blurred. Depending on daily political requirements and opportunities, certain indicators and data could be weighed differently; the programme could be politically instrumentalised in a way that does not correspond to the overall objective and the self-organising rationale of the programme. Thus, all data collected should be in connection to the explicit objectives of the programme.

A comprehensive monitoring could deteriorate to an "l'art pour l'art" exercise, either producing monitoring fatigue or wasting important human and financial resources. The best means to avoid this is to connect the monitoring not only to the strategic objectives of the programme, but to the strategic planning of the structures / centres themselves. Once the monitoring activity is not only a top down instrument but at the same time accepted as a necessary strategic tool, fatigue and waste can be minimised.

Monitoring systems tend to neglect context variables as they are mechanistic in nature. A "naive" benchmarking approach through which many different projects and structures are compared on the basis of uniform data sets is counterproductive. Monitoring systems must strike the balance between uniformity / comparability on the one hand and indicators that map the context. Ideally, monitoring reports are accompanied by some interpretative exercise by the

27 Evaluation should be independent from the monitoring and should, ideally, serve to improve the monitoring process. An ex post evaluation should not only use the data delivered by the monitoring, but also verify and more importantly - contextualise it.

${ }^{28}$ See also the notion of a fifth level governance in chapter 8 
management of projects, respectively the centre directors, as well as by the programme management.

\subsubsection{Evaluating MAPs: What do we measure}

Starting with an evaluation of a MAP the very first question we have to ask is what we are intending to measure at all. One of the most intuitively natural ways to evaluate a simple intervention is through a chain-link model. A similar approach is the distinction between output, outcome and impact, where outputs are immediate technical results like software tools, publications etc. outcomes are the direct effects of the intervention like new jobs created, increased productivity etc. and impacts are the wider effects of the programme on the society, e.g. faster diffusion of technology, changing innovation behaviour (see e.g. OECD 1997, Ohler / Arnold et. al. 1998, Kuhlmann / Bührer 1999).

One more concept which has taken into account is the concept of social returns: The return of research activities by a firm consists not only of the private return the firm receives but also of the knowledge spillovers and market spillovers the innovation causes. The following picture shows the complete social return of such an investment:

Exhibit 19: Social return of investment

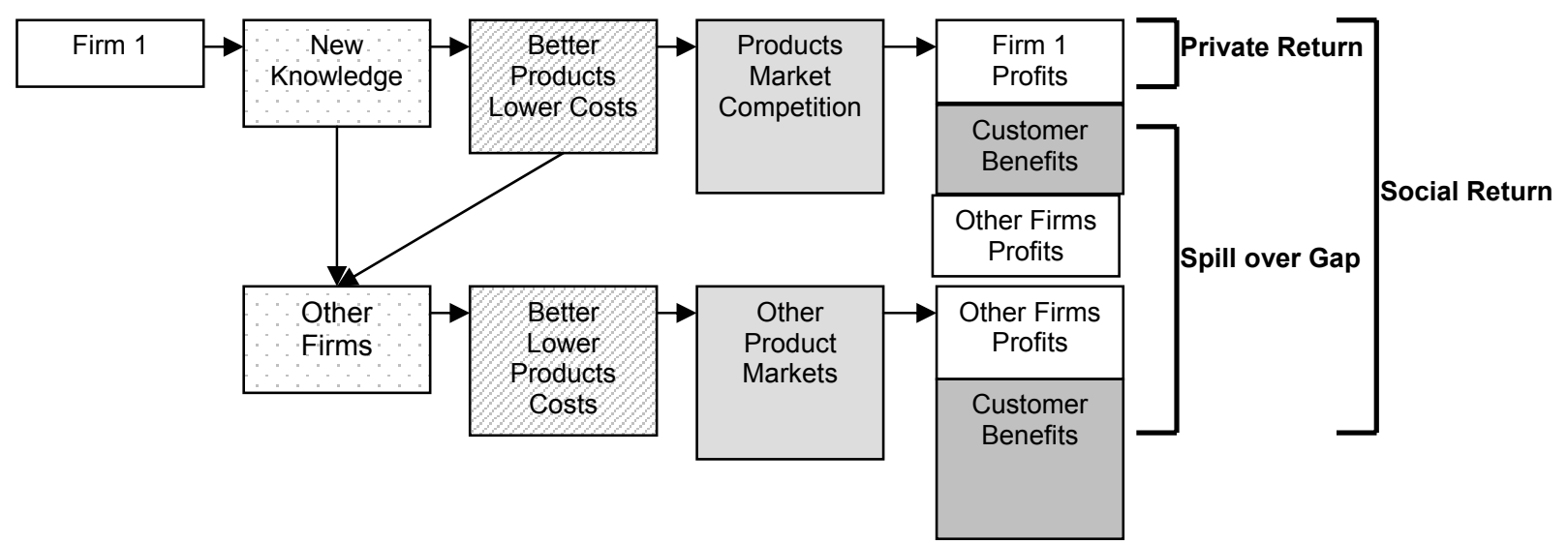

The further we look, the more factors outside of the programme in question come into play and the more complex is measurement. The measurement of impacts does not only want to capture all the direct and indirect effects of an intervention but all the effects on the whole society. Difficult enough for "normal" RTDI programmes, for MAPs the picture becomes even more complex. As discussed above, we could do better to think of impacts as mediated by a collective, than as a series of impulses. 


\subsubsection{Evaluating MAPs: How do we measure?}

Concerning tools and techniques, a lot of different approaches are available for impact assessment. In this section, we briefly review and systematise them and discuss their usefulness in the context of evaluating MAPs.

Following the categorisation of the EPUB project ${ }^{29}$ three big method-groups can be distinguished: (i) Statistical data analysis; (ii) Modelling methodologies; (iii) Qualitative and semi-quantitative methodologies.

We will explain these different approaches in some more detail by the following tables:

Exhibit 20: $\quad$ Statistical data analysis

\begin{tabular}{|l|l|l|l|l|}
\hline Method & Description & Impact relevance & Strength & Weakness \\
\hline $\begin{array}{l}\text { Innovation } \\
\text { Surveys }\end{array}$ & $\begin{array}{l}\text { Provide basic data to } \\
\text { describe the innovation } \\
\text { process, summarised } \\
\text { using descriptive } \\
\text { statistics. }\end{array}$ & $\begin{array}{l}\text { "Faster diffusion of } \\
\text { Innovation" }\end{array}$ & $\begin{array}{l}\text { Permits to identify size } \\
\text { and distribution of } \\
\text { impacts }\end{array}$ & $\begin{array}{l}\text { Costs, some types of } \\
\text { information are difficult } \\
\text { to obtain }\end{array}$ \\
\hline
\end{tabular}

\section{Exhibit 21: $\quad$ Modelling methodologies}

\begin{tabular}{|c|c|c|c|c|}
\hline Method & Description & Impact relevance & Strength & Weakness \\
\hline Micro Models & $\begin{array}{l}\text { permit to study the } \\
\text { effect of policy } \\
\text { intervention at the } \\
\text { level of individuals and } \\
\text { firms. }\end{array}$ & \multirow[t]{2}{*}{$\begin{array}{l}\text { "Firms competitiveness", } \\
\text { "Regional, country } \\
\text { productivity" } \\
\text { "Employment" }\end{array}$} & \multirow[t]{2}{*}{$\begin{array}{l}\text { R\&D Additionality, } \\
\text { Social rate of return to } \\
\text { R\&D } \\
\text { Long term policy } \\
\text { intervention impact }\end{array}$} & \multirow[t]{2}{*}{$\begin{array}{l}\text { Data demanding } \\
\text { Disclose information, } \\
\text { Robustness, } \\
\text { time lags }\end{array}$} \\
\hline Macro models & $\begin{array}{l}\text { allow estimating the } \\
\text { broader socio- } \\
\text { economic impact of } \\
\text { policy interventions. }\end{array}$ & & & \\
\hline $\begin{array}{l}\text { Control group } \\
\text { approaches }\end{array}$ & $\begin{array}{l}\text { allow to capture the } \\
\text { effect of the } \\
\text { programme on } \\
\text { participants using } \\
\text { statistical sophisticated } \\
\text { techniques } \\
\end{array}$ & $\begin{array}{l}\text { Firm, industrial } \\
\text { competitiveness }\end{array}$ & $\begin{array}{l}\text { Capture the impact of } \\
\text { policy intervention on } \\
\text { the programme } \\
\text { participant entity }\end{array}$ & Costs, Data Demanding \\
\hline
\end{tabular}

\footnotetext{
${ }^{29}$ EPUB - Socio Economic Evaluation of Public RTD Policies: EPUB - Tool Box (June 2002)
} 
Exhibit 22: Qualitative and semi-quantitative methodologies

\begin{tabular}{|c|c|c|c|c|}
\hline Method & Description & Impact relevance & Strength & Weakness \\
\hline $\begin{array}{l}\text { Cost Benefit } \\
\text { Analysis }\end{array}$ & $\begin{array}{l}\text { Allows establishing } \\
\text { whether a programme / } \\
\text { a project is } \\
\text { economically efficient } \\
\text { by appraising all its } \\
\text { economic and social } \\
\text { effects. }\end{array}$ & $\begin{array}{l}\text { "Quality of life", } \\
\text { "Standards of living" }\end{array}$ & $\begin{array}{l}\text { Socio economic effect } \\
\text { of the intervention }\end{array}$ & $\begin{array}{l}\text { depends largely on the } \\
\text { assumptions made } \\
\text { Data demanding }\end{array}$ \\
\hline \begin{tabular}{|l|} 
Expert Panel \\
Peer Review
\end{tabular} & $\begin{array}{l}\text { Measurements relying } \\
\text { on the perception } \\
\text { scientists have of the } \\
\text { (scientific) contribution } \\
\text { made by others. }\end{array}$ & R\&D Performance & $\begin{array}{l}\text { Flexibility, Evaluation of } \\
\text { scientific merits }\end{array}$ & $\begin{array}{l}\text { Economic benefits not } \\
\text { captured }\end{array}$ \\
\hline \begin{tabular}{|l} 
Field Studies / \\
Case Studies
\end{tabular} & $\begin{array}{l}\text { Use direct observation } \\
\text { of naturally occurring } \\
\text { events to investigate } \\
\text { behaviours in their } \\
\text { indigenous social } \\
\text { setting }\end{array}$ & $\begin{array}{l}\text { "Quality of life", } \\
\text { "Organisational } \\
\text { Efficiency", "Industrial } \\
\text { Competitiveness" }\end{array}$ & $\begin{array}{l}\text { understanding how } \\
\text { contexts affect and } \\
\text { shape impacts }\end{array}$ & $\begin{array}{l}\text { results can't be } \\
\text { generalised }\end{array}$ \\
\hline $\begin{array}{l}\text { Network } \\
\text { analysis }\end{array}$ & $\begin{array}{l}\text { Social network research } \\
\text { serves to analyse the } \\
\text { structure of } \\
\text { (cooperation) } \\
\text { relationships and the } \\
\text { consequences for } \\
\text { actors' decisions on } \\
\text { actions. }\end{array}$ & $\begin{array}{l}\text { High. SNR is an key } \\
\text { method to assess e.g. } \\
\text { "behavioural } \\
\text { additionality". }\end{array}$ & $\begin{array}{l}\text { it can point to weak } \\
\text { spots in the } \\
\text { communication and } \\
\text { cooperation of network } \\
\text { members; } \\
\text { the analysis of total } \\
\text { networks provides a } \\
\text { multiplicity of results, } \\
\text { by comparing different } \\
\text { network types it is } \\
\text { possible to derive } \\
\text { specific success factors; }\end{array}$ & $\begin{array}{l}\text { Time consuming, } \\
\text { especially for the } \\
\text { questionees } \\
\text { data security } \\
\text { tendency to } \\
\text { overemphasise the } \\
\text { visualization of results }\end{array}$ \\
\hline \begin{tabular}{|l} 
Foresight / \\
Technology \\
assessment
\end{tabular} & $\begin{array}{l}\text { Used to identify } \\
\text { potential mismatches in } \\
\text { the strategic efficiency } \\
\text { of projects and } \\
\text { programmes }\end{array}$ & \begin{tabular}{|l|} 
Technological \\
paradigms shifts
\end{tabular} & $\begin{array}{l}\text { Assessing strategic } \\
\text { impact }\end{array}$ & $\begin{array}{l}\text { Impossibility to detect } \\
\text { major RTD } \\
\text { breakthroughs }\end{array}$ \\
\hline Benchmarking & $\begin{array}{l}\text { Allows to perform } \\
\text { comparisons based on } \\
\text { a relevant set of } \\
\text { indicators across } \\
\text { entities providing a } \\
\text { reasoned explanation of } \\
\text { their values }\end{array}$ & $\begin{array}{l}\text { Industry } \\
\text { competitiveness, good } \\
\text { governance }\end{array}$ & $\begin{array}{l}\text { Support to systemic } \\
\text { evaluation of institutions }\end{array}$ & Non transferable \\
\hline
\end{tabular}

Due to the complex structure of MAPs and due to the importance of learning elements, some methods are of specific importance in the context of the evaluation of MAPs. Nevertheless, methods have to be adapted to the specific network character of MAPs and the use of multiple parallel methods remains important. This may be exemplified by the Peer review method and its relevance in the context of MAPs, since this is the method mostly applied by the network members.

Peer evaluation is based on scientists' perceptions of contributions by others and is influenced partly by the magnitude of those contributions and partly by other factors. It covers a wide range of methods in which peers express opinions. However peer review needs to be guided and modified according to the particular evaluation's objectives and methods. Peers are important in research quality evaluation since they represent the key reference point: namely, the state of the art of the given scientific field. Peer review is one of the most common evaluation techniques. Many government science and technology funding agencies use peer review as a primary instrument of evaluation. 
Peers are important contributors in evaluation, since they are the experts and remain the most reliable, 'rounded' source establishing scientific quality. There is a risk that peers' evaluation is biased, though this can be reduced by using structured guidelines, evaluation strategy and strict evaluation management. In the context of MAPs, Peer review techniques have to go beyond quality of science and include issues concerning a broader context of social and economic benefits. These techniques are described as modified or extended peer review. In this type of evaluation, scientific experts generally need professional support from the evaluators and from other non-peer members in the expert group - such as social scientists, economists or business people, and potential users - to be able to assess outcomes and impacts. There are also combinations of peers with other evaluation methods. Modified / extendet peer review's key weakness is the small number of individuals who can be involved. In many types of evaluation, and especially for the evaluation of MAPs it is necessary to use complementary techniques in order to compensate for this.

Apart from finding the right mix and adaptation of evaluations methods in order to capture MAPs there are two remaining problems: time and persuasion. Since many important features of MAPs may take years to have effects (most of socio-economic impacts, capacity building, longterm change of behaviour, etc.), a long term evaluation perspective should be applied. This long term perspective contrasts with the need of policy makers for quick arguments and results. Furthermore, policy makers - and partly the people working in the centres and networks too used to be unhappy with comprehensive and expensive evaluation designs.

\subsubsection{Evaluation: Learning and formative approaches}

Sadly, the idealised picture of the R\&D policy making system shown below is often no more than an ideal. In this wider context, evaluation is expected to provide accountability, learning, policy guidance at all three levels. Of course, there should be a vertical coordination between these levels. In real life, evaluation - and the necessary complementary policy analysis capabilities are not always so widely distributed.

An important implicit characteristic of the hierarchy shown is the idea that policies can simply be decomposed into programmes and projects - an idea which itself is problematic in a systems world. 


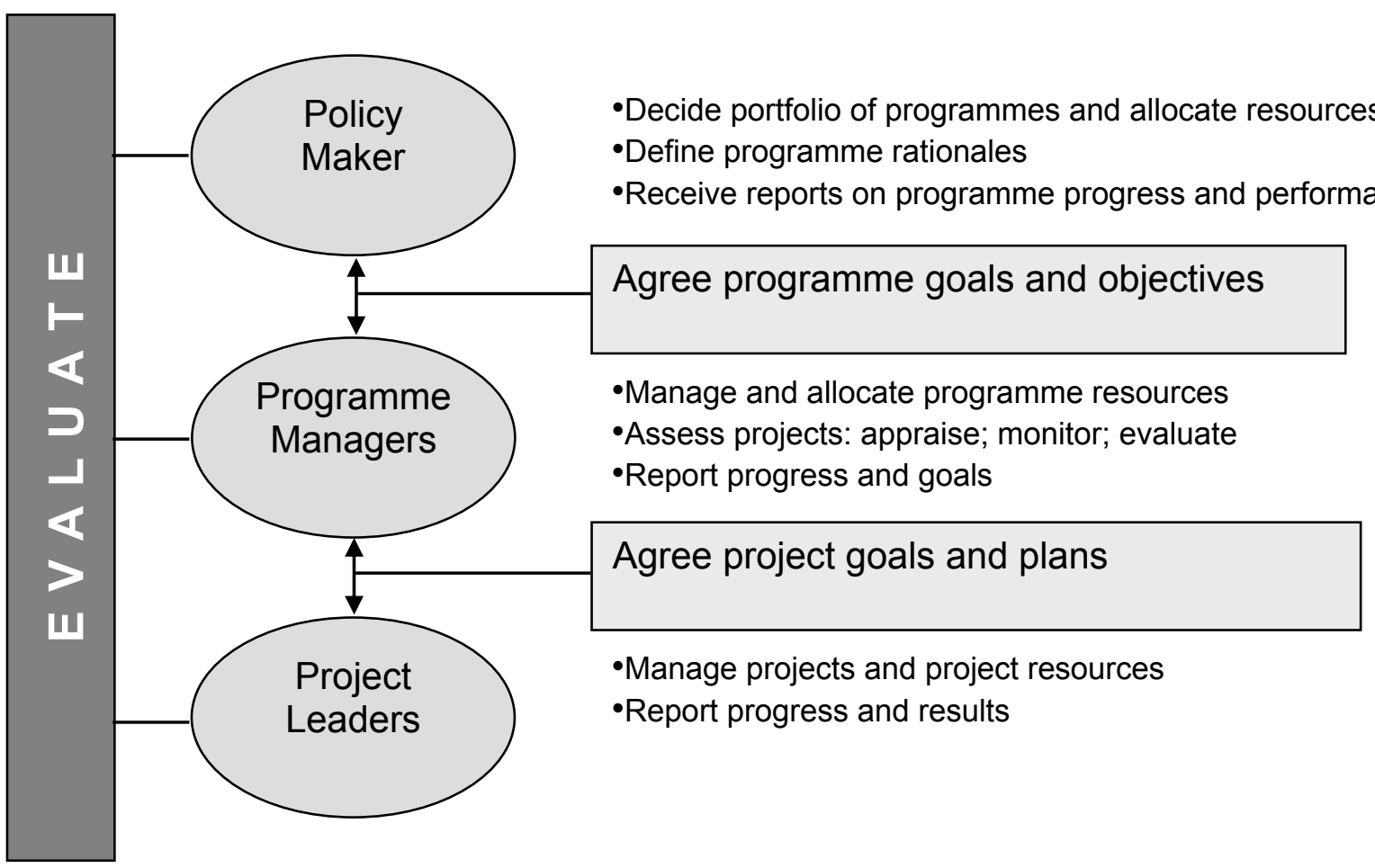

Source: Arnold / Boekholt / Keen (1995)

Learning from evaluations of MAPs needs to be codified in at least two ways. One is via changes in processes at the level of MAP funding and management. The second is in a more portable form, allowing the learning to be re-used in other situations and input to policy and intervention formulation. In the context of MAPs, the following functions seem to be especially important: (i) Improving programme management and „fine tuning” science and technology policy programmes, (ii) Releasing new ideas or legitimisation already circulating ideas about changes in R\&D centres and funding agencies, (iii) Improving transparency of the rules of the game of S\&T funding decisions and (iv) Enhancing the information basis for science and technology policies, in the sense of a government-led mediation between diverging and competing interests of various players within the science and technology system (compare also Kuhlmann 1999)

With regard to sophisticated programmes like MAPs which intend a structural and institutional change, evaluation procedures should be conceptualised and applied as 'learning medium' within Multi Actor-arenas. Such evaluation procedures are intended to be an 'intelligent' supplier of moderation strategies of the responsible political actors. 'Intelligent' means linking research and technology, industrial efforts, societal needs and political interventions more transparently and insofar easing the interactive collaboration between the parties involved. In that sense, 'intelligent' procedures of policy development shall supply information that: (i) Is based on analyses of the dynamics of changed research systems, innovation processes and functions of public policy; (ii) Take into account the different institutional and interest-related perceptions of the actors involved; 
(iii) Help making the different perceptions more 'objective' through offering compatible indicators and mechanisms of information dissemination, and (iv) Can be accompanied through discourses between the competing actors (or their representatives).

Such evaluation procedures of RTDI policy measures go much further then the simple measurement of scientific and technological performance; they are intended to contribute to the creation of new actor constellations and to the change of actor's perceptions.

The structural ambitions of MAPs raise at least two problems for programme design and learning through evaluation, however. Generally, it is seen as good practice to engage stakeholders or beneficiaries in programme design and to consult them in evaluations, since they normally bring unique knowledge and perspectives that are important for success. However, there can be an inherent conflict between the MAP agenda of structural change and the beneficiaries' interests in the status quo - which may, indeed include the problems tackled by the MAP (Braun 1993). A corresponding learning question is how the learning through MAP evaluation can cross boundaries, in other words how experiences of MAP can be taken up in different policy-making organisations, such as different ministries. How can learning mechanisms be put in place that will allow evaluation results to be 'invented here' and therefore 'accepted here'?

The expectations of the evaluation process veer between two functional poles: evaluation can serve primarily to measure performance and thus retrospectively justify promotional measures, i.e. the summative function, or it can be utilised as a learning medium, in which findings about the connections between cause and effects of current or completed measures are used as intelligent information for currently running or future initiatives, i.e. the formative function (see Bührer et. al. 1999).

The summative pole is above all at home in the evaluation practice of the Anglo-American countries: here in the efforts towards reform and cost reduction in the public sector ("New Public Management") procedures aimed at "Performance Measurement" exercised great influence also in research and innovation policy (Shapira et al. 1997). Sparked off by the "Government Performance and Results Act (GPRA)", the US government and a majority of the federal states pressed increasingly ahead with "performance-based management and budgeting systems" (Feller 2003).

As however MAPs are complex (set of diverging aims, effect on behaviour, affecting heterogeneous institutions etc.), summative performance measurement rapidly reaches its limits. For this reason formative, learning-oriented evaluation approaches were developed and applied - partly in competition, partly complementary to the summative ones. Proceeding from the experience that evaluation results often produce only small impacts in policy decisionmaking processes or only support few of the expectations and interest positions represented in a policy arena, evaluation experts tried (and increasingly also policy-makers) to loosen up / relax the borders between evaluation and decision-making processes, even to partially integrate the two spheres. 
The key word of the new, extended understanding /definition of evaluation is "negotiation" in actor arenas( Kuhlmann 1998): (i) Evaluation is conceived as a procedure of the empiricalanalytically prepared, structured presentation and confrontation of (partly conflicting) actor perspectives; the entire spectrum of evaluation methods can be utilised therein. (ii) The evaluator acts as a "facilitator", as a "critical friend", he supports the mediation/ moderation of the arguments in the negotiation system on the part of actors of the politico-administrative system. (iii) The evaluation target is not only the assessment of facts from a single actor perspective (e.g. the politico-administrative system), or the "objective" examination of the suitability of a policy, but the stimulation of learning processes by overcoming hardened actor attitudes.

Elements of system-oriented, formative evaluation:

- Identify governance of related arena: power distribution, formal and informal rules; stimulating and hampering factors for integration;

- MAPs need „multi-perspective approach“ of evaluation: identify and name the polyvalence of represented actor interests;

- Create appropriate indicators and benchmarks for system-orientation and networking - in agreement with policymakers and beneficiaries;

- Apply creatively variety of quantitative and qualitative analytical concepts and methodologies, e.g. social science network analyses as useful methodological tool;

- Analyse the cost of complexity:

Evaluation is about learning, and evaluating MAPs means to set up complex learning processes. Comprehensive evaluation systems of MAPs should try to integrate traditional evaluation approaches and these broader principles and opportunities of strategic intelligence in order to improve the overall learning process of policy makers and stakeholders of MAPs.

\subsection{Experiences and examples on Monitoring and Evaluation}

In contrast to the analytical inputs so far, which focused on the 'ought-to-be' aspects of MAP evaluation we will focus now on the 'real picture' in our MAPs. Based on questionnaires, sent to all the MAP-TN members, a summary on the topic of Monitoring and Evaluation was formulated with the intention to draw these two sides - the 'ought-to-be' and the 'real picture - together once they have been discussed by the network.

Note that this 'real picture' is in many countries just evolving, as MAPs are a rather young kind of funding programmes. This means lack of a long standing experience and of a fully fledged MAP evaluation culture. There are some countries nevertheless with ample experiences, among them AU and CA. As other MAP countries just now come into serious evaluations of their programmes and large project consortia, we have included the preceding conceptual considerations in this "monitoring and evaluation" chapter. 


\subsubsection{Monitoring}

The term "Monitoring" refers to the periodical collection of data and indicators concerning the performance of projects including the financial controlling of the projects.

As a general result, for all the MAP-TN members it can be stated that Monitoring is highly standardised and mainly quantitative. Fixed monitoring guidelines are part of the management of most MAPs, they are focussed on numbers, indicators and financial information, in some cases accompanied by qualitative parts while in other cases data management tools are used (ES, UK, HU, NL).

In $A U$ and in CA monitoring and evaluations are mandatory for all government funding programmes and were therefore set up at the very early beginning of the programme. In Australia the centre performance is monitored by a number of reviews by external experts and through reports submitted to the CRC secretariat (Quarterly financial statements, Annual Reports, Management Data Questionnaire). In CA the NCE directorates require Annual Reports consisting of bilingual corporate reports, statistical tables, financial reports, statements of other sources of funding, and administrative reports on an annual basis. The advantage is clearly for the project and programme evaluation, be it mid-term or ex post, as good and continuous data collection exists.

Box 24

\section{The Finnish Tekes Technology Programmes - Monitoring system}

The Tekes Technology programmes apply monitoring, which is a combination of standard reporting to Tekes (used in all Tekes funded projects) with some additional reporting to the programme steering group. The problem Tekes has encountered is not that a Technology programme would cause monitoring fatigue, but that at times research groups are flooded with information requests from several evaluations due to the fact that their funding is coming from several sources. Tekes monitoring consists of both quantitative and qualitative data.

Apart from this standard monitoring which looks quite similar for all the partners especially concerning the controlling function of the Monitoring system, two topics were discussed in some more detail: The question of additional qualitative information and the question of Monitoring fatigue. Additional information can be gathered in workshops meetings or cooperative boards. Nearly all the Competence Centre programmes have such boards (SE, AT, HU, AU) as a soft and qualitative monitoring and to some extent steering instrument. Note also the different approaches to organise information exchange between centres in these countries. They meet often with the funding organisation as guest or even host - for information and best practice exchange. 
"Boards" as a qualitative steering element in addition to the standardised monitoring system The Case of the Austrian K plus Programme

"Boards" are periodical meetings of all the partners involved in a K plus centre, including the funding and managing organisations. Boards are no legal bodies according to Austrian corporate law, but due to the strategic orientation - the board makes decisions or recommendation on issues like budget, new projects, and new partners - the board has some informal power. Intended as forums for selforganisation of centres, the boards are successful in creating common understanding and cooperative culture. The programme manager TIG participates in the boards as a non-voting member and takes a role as advisor, as observer or as consultant.

From the point of view of the programme manager, boards have been established as an important instrument for monitoring of culture, behaviour and ongoing developments. Especially in combination with other more quantitative monitoring it provides a very good information base. One major challenge is the balance between a purely passive attitude and a too strong steering behaviour. Another challenge is the handling of vested interest: Participants often pursue individual interests, less often the interest of the centre. Conclusion: Valuable instrument, but use with care.

One more major topic of discussion was the question of monitoring fatigue. Although most of the programmes employ modest monitoring systems, HU, ES and esp. SE, AU and CA mention the point of the „burdensome financial audit" or the threat of „monitoring fatigue“. CA's NCEs complained about the high reporting load which was quite often required by the network managers and not by the programme management, the NCE directorate.

Monitoring fatigue is especially a problem if the system is neither transparent nor user-friendly. This is especially the case, if collected data are difficult to gather for the projects and centres, and if same data collected more than one time for different purposes, and if no one has any idea what happens with the enormous amount of data collected. MAPs with no problems concerning monitoring fatigue employ good, relatively simple and robust systems. One special case to mention in this context is the DE with its $\mathrm{CCN}$ programme, where the monitoring system for R\&D projects in the context of the $C C N$ is the one which is used for all R\&D grants in Germany. Therefore, it is well known to the researchers from university and industry, not too exhaustive but fulfils the requirements to assure accountability.

To avoid monitoring fatigue, a general rule should be applied: Collect all data you use and use all data you collect.

In conclusion, monitoring strengths of most of the MAPs include: Good financial and milestone data, which facilitates management of centres and projects. The collection of data allows the generation of reports on demand concerning all financial aspects that feeds into evaluations and other ad hoc requests. The bureaucracy and burden for the projects and centres is bearable. Monitoring weaknesses include: The systems are less flexible when it comes to generating reports on qualitative matters (here a construction similar to the mentioned "boards" can be helpful). In most cases, outcome and impact data are not fully reliable. The monitoring systems are backward oriented and can serve as a basis for decisions on further developments only in a limited form. 


\subsubsection{Project evaluation}

"Project Evaluation" is defined as an ex ante, interim or ex post judgement about the quality (performance, scientific quality, impact etc.) of projects or centres and in most cases it is carried out by external experts ${ }^{30}$. Remember that in this context a consortium, a Competence Centre or a Cluster initiative is seen as one project!

Whereas all MAPs involved in the network have experiences in the ex ante evaluation (see chapter 5 on selection procedures), this is not the case for interim and ex post evaluations. In some cases (HU and EE and regarding ex post evaluations AT and SE) programmes are comparatively new and projects run for a long time, so that some evaluations are planned but not carried out yet. In other cases such interim and ex post evaluations are not even planned (e.g. SL with a totally and UK with a partly missing interim evaluation or SE and AT were the decision about an ex post centre evaluation is still open). Note the very open and publicly available Swedish mid term Centre evaluations.

The picture is somehow different for the StarMAP countries as we looked at mature programmes. Again two prominent examples: AU carries out three reviews of the CRC Competence Centres in the seven year funding period: first year visits and second and fifth year visit whereas the latter decide about the continuation of funding. A number of reports are already available. In CA after 4 years a mid-term review of the NCE Networks of Excellence is carried out, for which each network has to prepare a detailed report on the first years of activity and a strategic plan for the remaining years. The mid-term review could result in continued funding, continued funding on a conditional basis, or the phasing out of a network before the end of the current award.

\section{Box 26}

\section{The Norwegian TEFT programme: Being hardly a MAP allows minimum requirements}

This programme runs totally different as its aim is to stimulate cooperative research activities of SMEs and tries therefore to reduce the administrative as much as possible: Therefore a Technology Attaché carries out a technical verification of the project. And instead of a continuous monitoring SMEs have to do a voluntary self-evaluation filling in forms provided by the programme management. The technical verification of the project is basically up to the company, which gives its approval or disapproval based on the technology project report at the end of the project. If a project is not approved and subsequent improvements do not change this, the second half of the money might not be paid. But this has never been the case until now. The performance by the company itself is not evaluated.

\footnotetext{
${ }^{30}$ The exception is FI where Tekes internal experts carry out the ex-ante project evaluation and also NO where the key person is the Technology Attaché.
} 
One of the most interesting results of the comparison of the different designs of project evaluation is that the approaches are very similar concerning the ex ante evaluation but totally different concerning ex post or interim evaluation which is evident in particular from the mature StarMAP examples. For the ex ante evaluation, the selection of the funded projects peer review methods or modified peer review methods were used by all MAPs. For the interim and ex post evaluation the spectrum of designs applied reaches from extended monitoring processes (ES and $D E$ ) to sophisticated peer-based approaches (for the interim evaluation AT, SE, AU and CA).

One major reason for the different approaches is the difference between the MAPs which indeed explains a lot of divergence in the project evaluation designs is the scale and complexity of the project. Competence Centre programmes, where the unit of investigation is a whole centre or big network including all complex relationships between science and industry in a nutshell, of course tends towards a more complex evaluation design ${ }^{31}$ than project based programmes (as we can find for example in the UK or SL).

One more explanation for the differences is the kind of monitoring employed. If the monitoring system is a comprehensive one, fulfilling nearly all the information needs we have concerning the quality of the projects, no specific project evaluation would be necessary. This is for example the case in ES, the opposite case could be identified in SE, where a modest monitoring system goes along with a comprehensive project evaluation system.

Other crucial points, which explain much of the differences in evaluation design is the evaluation and agency culture in a country, including the evaluation competence of those commissioning evaluations and the extent of experience in using MAPs in the country.

${ }^{31}$ Germany is an exception: In spite of the complex structure of the Nanotechnology Competence Centres, no specific project evaluation takes place; the interim evaluation is a simple audit and can be interpreted as an extended monitoring exercise. The reason here is the existence and the specific design of an accompanying programme evaluation which focussed exactly on the specific architecture of the Competence Centres and their internal and external relationships. 


\section{Comprehensive stage-by-stage evaluation on centre level - The Case of the Swedish Competence Centre Programme}

The Swedish system consists of three interim evaluations during the lifetime of a centre:

Year 2: Performance (MAP-specific);

Year 5: (Mid-term): Performance + scientific output;

Year 8: Scientific and technical output + industrial impact + future strategies.

The evaluations were carried out by international review teams (a core group + scientific peers) during two-days site visits. Evaluation results are public. As far as experiences go now the evaluations are established as a very successful measure for development of the Centres and the Programme! It has to be mentioned that the first evaluation after $1 \frac{1}{2}$ year gives a very good and early inside into the performance of the centre. Training programmes for Centre managers were established as a direct result.

One more important question was whether MAP specific features were taken into account, and if, how exactly this works. Concerning the integration of learning elements and feed-back loops, all programmes with interim evaluations or accompanying programme (e.g. AT, SE, DE, AU and $\mathrm{CA}$ ) evaluations employ a dialogue-oriented design where not only scientific quality of the work done in projects and centres but also the connection between the different worlds as well as their organisation and management is addressed. This is mainly done by integrating systemknow-how into the evaluation process and thus increases the strategic intelligence of the system.

Conclusions: After taking a careful look at the specific background of the programmes and the governance structures of the countries, one cannot give a simple recommendation which kind of project evaluation should be employed. Different "good practices" are possible, if it is guaranteed, that monitoring, project evaluation and programme evaluation are connected in a proper way enabling the important decisions about further developments of the projects and the programme as a whole.

\subsubsection{Programme Evaluation}

"Programme Evaluation" is defined as an ex ante, interim or ex post judgement about the quality (performance, scientific quality, impact etc.) of a whole programme and in most cases it is carried out by external experts.

Due to the fact that most of the MAPs represented in the network are relatively new programmes, concrete experiences concerning interim and ex post programme evaluation are modest. Indeed only for three programmes, the German Nanotechnology CC programme, the UK Partners in Innovation and the Slovenian Toolmaker's programme, programme evaluations were already carried out. One more problem is that the few approaches we can witness in the MAP-TN differ extremely. Nevertheless we can subdivide the approaches into three types (which perhaps could stand for further examples): 
The first approach designs programme evaluation with a focus on socio-economic impact evaluation. This is the case for SE, where the design is currently under construction, for EE as a plan in the future and to some extent for AT, where a mainly qualitative "assessment" was carried out recently and a more impact-oriented evaluation is planned for the next future. Since in all these programmes evaluations at centre level took place with a comprehensive qualitative design, quantitative measurement is left for the programme level, though AT already collects impact measures for a future programme evaluation. The UK example could be taken under this category too, since programme evaluations are mainly impact oriented but in contrast to the other cases the connection between project evaluation and programme evaluation is not a systematic one.

The second approach is the German one with modest project level evaluation but an accompanying formative programme evaluation, focussed on learning aspects and with explicit integration of specific methods like Social Network Analysis. With regard to sophisticated programmes like the Nanotechnology Competence Centres which intend to bring about a structural and institutional change, evaluation procedures can be - and were - were conceptualised and applied as a "learning medium“ within Multi Actor arenas (Kuhlmann 1998). Such evaluation procedures are intended to be an "intelligent" supplier of moderation strategies of the responsible political actors. "Intelligent“ means linking research and technology, industrial efforts, societal needs and political interventions more transparently and insofar easing the interactive collaboration between the parties involved. Here again, the connection between project and programme evaluation doesn't exist. 
Evaluation as a "learning medium“ within Multi Actor arenas in the German Nanotechnology Competence Centre Programme

The programme evaluation is conducted by two different kind of organisations: a research institute (Fraunhofer ISI) and a business consultancy (mundi consulting). The evaluators were free to choose the most appropriate design and methods, this was subject of the selection process. Objectives of the evaluation are (i) the assessment of the impact and level of goal attainment of the scheme three years after the start, illustrated by the six promoted nanotechnology Competence Centres, and (ii) the stimulation of learning experiences and optimization processes through the collection of experiences and the intensive exchange among all parties involved. According to these different objectives the evaluators have two distinct roles: they should act as a "critical friend" as well as an "objective assessor". In terms of methodology, the design of the evaluation study foresees (i) a strategic analysis of the scheme, (ii) an in-depth-analysis of the six promoted CCN for nanotechnology, (iii) an analysis of alternative $\mathrm{CCN}$ models (in the field of nanotechnology, other fields of technology, national as well as international).

With regard to the schedule, the study is divided into three phases: preparatory phase (about 6 months), monitoring phase (18 months), evaluation phase (6 months). During the preparatory phase, the ancillary conditions are investigated, including the scientific and technological competences of the actors involved, the aims and strategies of the centres and the organisational models developed in order to achieve these aims. The monitoring phase is characterised by a detailed analysis of the structures of the (internal and external) cooperation and communication and a comparison with alternative models of Competence Centres - at the national and also the international level. Finally, the evaluation phase is devoted to the analysis of a number of quantitative and qualitative indicators referring to nine performance dimensions (scientific performance, competence with regard to collaboration, interdisciplinarity, technological competence/ transfer, efforts to influence the business environment, education and training, public relations, norms and standards, long-term perspectives of the centres).

Overall, different kinds of methods were used: document analysis, qualitative interviews, innovation studies, bibliometric analysis, control group approach, field / case studies, network analysis and workshops to discuss / adapt and disseminate the results.

A third approach is represented by Slovenia in the MAP-TN: Here the line between project evaluation and programme evaluation is blurred, so programme and project evaluation use more or less the same methods and criteria.

\subsubsection{StarMAP examples with its mature programmes provide a different picture}

The French PREDIT transport research programme carried out and will carry out the following ex post evaluations: The first PREDIT (1990 - 1994) had a final evaluation by external evaluators in 1995; PREDIT 2 (1996 - 2000) had a mid-term evaluation by external evaluators, which took place in the end 1998, early 1999. It was followed by an ex post evaluation by an independent expert committee supported by professional research evaluation experts, 2001. For PREDIT 3 (2002 - 2006) a mid-term evaluation and ex post evaluation are foreseen, a "cellule de qualité" was installed for ongoing quality monitoring. 
In the Dutch TS Programme, every four years, an ex post evaluation by external evaluators, which serves to regulate the internal reviews deriving from the project level evaluations. The evaluators are chosen through competitive selection. The last evaluation was the evaluation of the BTS in 2000, carried out by a Dutch policy consultant. The aim of the ex post evaluation is to assess the programme's effectiveness. It also serves a learning purpose: the results of the evaluation are used to adjust the programme and/or the management.

Within the Norwegian TEFT programme, evaluation is not a central issue, since the budget is rather small and the quality or outcome of a project is not in the centre of attention. TEFT II will be evaluated ex post. The objective of the evaluation is to resume the experiences and learning effects achieved in the programme.

All Finnish Technology programmes are evaluated at least once during their lifetime. This used to be ex post and sometimes also interim. Generally it is the aim of the evaluation to provide feedback on how the programme objectives have been realised, to find out how relevant the programme is and to produce information to support the strategic development of programme activities and the activities of TEKES in general. The concrete goals again depend on the decision of the programme management and the evaluator. Today, Technology programme evaluation has been developed from evaluating single programmes into the direction of thematic evaluations, where the contribution of several programmes to thematic overall objectives is evaluated. These new types of thematic evaluations include e.g. "Climate change - Impacts of technology policy and programmes", "Targeted Technology Programmes: A Conceptual Evaluation - Evaluation of Kenno, Plastic Processing and Pigments Technology Programmes.", "Towards a competitive cluster - An evaluation of real estate and construction technology programmes" and "Space technology programmes 1995-2000." This means that some programmes can be evaluated one or more times during and/or after its lifetime, depending on the timing of specific thematic evaluations. At the same time, programme steering groups have been encouraged to strengthen their interim evaluation activities, which in some cases employ external experts and typically also strategic forward looking elements.

Evaluations in the Australian Cooperative Research Centres Programme are mandatory for all government funding programmes and agencies since 1990. The CRC programme has been under review several times, the two most significant evaluations being conducted in 1995 and 1998. Their conclusions amongst others were "that the CRC Programme become a continuing key element in the Government's approach to supporting education, training, research and development" but that "only a few centres will be likely to become self funding on the basis of their research".

In the Canadian Networks of Centres of Excellence Programme the first evaluation already took place in 1993, already 4 years after the program started. This exercise concentrated on management issues in NCEs which made evident that management varied from network to network, from badly to very well managed networks. It happened once that a resubmitted network for the second term was not taken again due to bad management even if there was a star-researcher involved. The second evaluation in 1998 focused on the impacts of the programme. The evaluators looked at several completed projects and carried out a "partial 
benefit/cost analysis" where the data collection turned out to be frustrating in particular in companies as they very reluctant to present figures, as e.g. on sales. Through interviews and the structure of the NCE-projects it was evaluated if real or fake collaboration took place. The third evaluation in 2002 on the rationale of the program summarised that the NCE program is seen "still as a valuable and unique part of the Canadian research landscape" and gave a strong recommendation to continue the program. The evaluation methodologies included International literature review, a review of the NCE performance data and other reports, Survey of all network partners through an e-mail based survey, interviews with the Network Scientific Leaders and Network Managers and web-based survey of Network Researchers, finally interviews with members of the Expert Panels and NCE Selection Committee.

As shown above the StarMAP countries have gained much more experience in particular on programme evaluation which is evident as most of these programmes run for more then a decade meanwhile.

\subsection{Lessons from the MAP-TN and StarMAP}

Coming to an end we want to go back to the very start of our work, were we tried to figure out which concrete characteristics of MAPs (as defined by the MAP-TN) have which consequence on the evaluation. Most important seems to be the proper integration of formative evaluation approaches based on a judgement of the network members.

The following picture can give an outlook on the further challenges in the field of evaluation of some of the MAP-TN programmes: The common understanding that evaluation has to be a strategic exercise, which needs a lot of professional know-how as well as a professional and competent management for the sound implementation of evaluation results. One more point is that a lot of MAPs struggle with the problem of vested interest: There is an certain need for more independency, transparency and publicity of evaluation results.

\section{Exhibit 25: Conclusions and outlook}

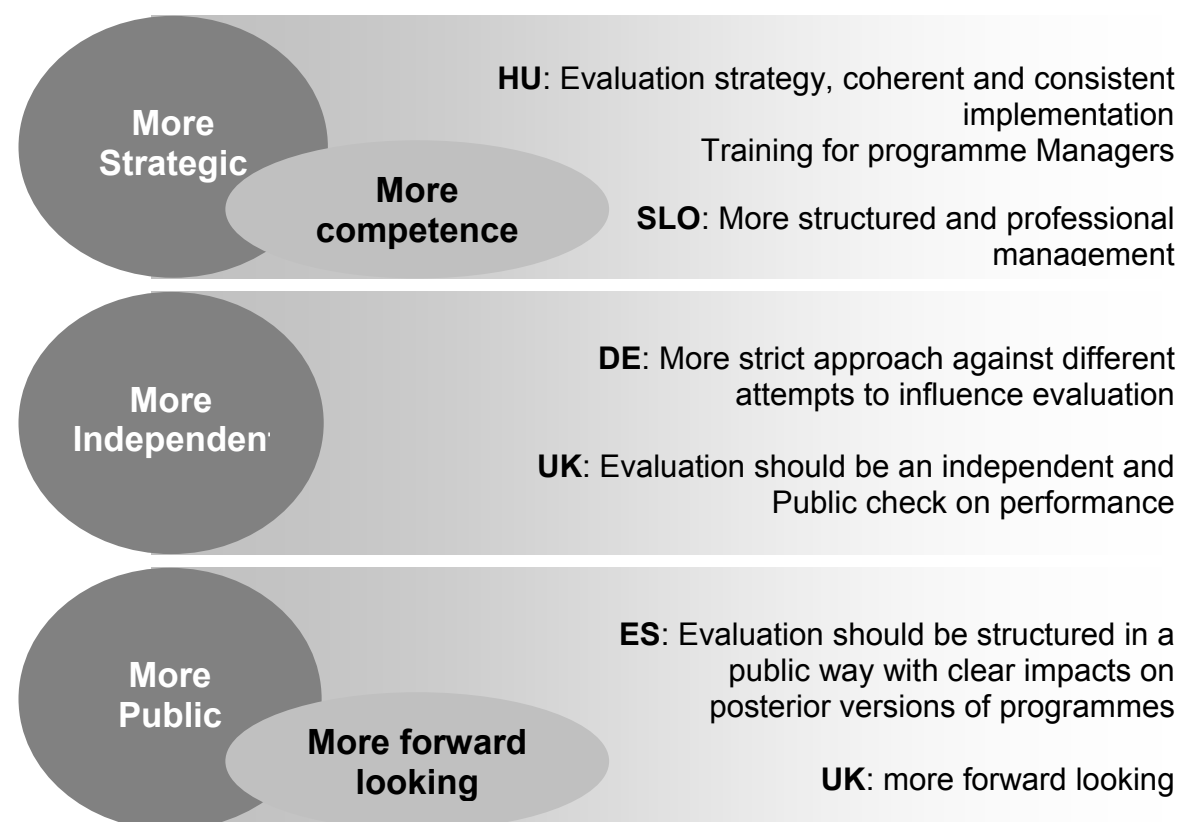




\subsubsection{Monitoring:}

How to select data and indicators properly, especially concerning the long time horizon MAPs usually have? The data needs to be collected on the level of projects (centres etc.) but should be aggregated currently. Once implemented, broader changes of the monitoring system should be avoided, since the comparability of data must be guaranteed over a long period of time. Monitoring should be designed in order to enable the analysis along different dimensions of importance for the funding body, such as the allocation to technological areas, regions, types of actors etc.

How to avoid monitoring fatigue? Same data for different purposes e.g. monitoring, evaluation, controlling should be collected only once and then distributed for the different uses. Too comprehensive data collection should be avoided: Good monitoring systems follow the principle: "Collect all data you use and use all data you collect".

How to use monitoring data as an input for evaluation? A monitoring system should deliver data for an accompanying, periodic or ex post evaluation of the projects / centres and the programmes as such. This improves the adequacy, effectiveness and efficiency of the data gathering of the evaluation. The monitoring data are at the same time important for the programme management/ agency for the optimisation of the programme management and the accompanying service/coaching activities.

How could qualitative information be added to monitoring systems? Monitoring systems in general are less flexible when it comes to generating reports on qualitative matters. Here a qualitative steering element in addition to the standardised monitoring system like Competence Centre "Boards" can be helpful.

\subsubsection{Project and Centre Evaluation}

How could learning elements and feed-back loops be integrated into the Evaluation process? The integration of learning elements and feed-back loops is of specific importance in the context of Competence Centre programmes, where the unit of evaluation is a whole complex centre with different research cultures working together. Here, evaluations should employ a dialogueoriented design where not only scientific quality of the work done in projects and centres but also the connection between the different worlds as well as their organisation and management is addressed. This could be done by integrating system-know-how into the evaluation process and thus increases the strategic intelligence of the system. A lot of emphasis should be given to the interim evaluation since only with interim approaches learning during the lifetime of the centre is possible.

How to cope with the specific challenge that not only scientific quality but also the cooperation of different organisations in the network or centre should be taken into account? By using peer review techniques, one has to go beyond quality of science and include issues concerning a broader context of social and economic benefits modified or extended peer review. In this type of evaluation, scientific experts generally need professional support from the evaluators and from other non-peer members in the expert group - such as social scientists, economists or business people, and potential users - to be able to assess outcomes and impact. 
Modified peer review's key weakness is the small number of individuals who can be involved. Therefore, it is necessary to use complementary techniques in order to compensate for this.

\subsubsection{Programme Evaluation}

How to use new formative approaches in order to enhance learning in the whole system? Since MAPs intend a structural and institutional change, evaluation procedures should conceptualised and applied as a learning medium within Multi Actor arenas. Such evaluation procedures are intended to be an 'intelligent' supplier of moderation strategies of the responsible political actors. 'Intelligent' means linking research and technology, industrial efforts, societal needs and political interventions more transparently and insofar easing the interactive collaboration between the parties involved.

Which methods are of specific importance due to the complex structure of MAPs and due to the importance of learning elements in the context of the evaluation of MAPs? Important methods include the proper application of peer review, Social Network Analysis (SNA) and some moderate impact assessment approaches. SNA is an expensive and time consuming tool, but nevertheless useful if applied properly. Peers are important contributors in evaluation, since they are the experts and remain the most reliable, 'rounded' source establishing scientific quality.

\subsubsection{Evaluation in the Policy Cycle}

What should be done to implement evaluation results properly and optimise learning out of evaluations? Evaluation results should, directly or indirectly, support decision-making processes in research and technology policy. This, in turn, requires that evaluation results be presented in a timely and effective manner and that sufficient scope is given to communicate them. The credibility and effectiveness of evaluations will, generally speaking, be enhanced by disseminating them widely and ensuring a suitable level of publicity.

Should evaluation be combined with other elements in order to improve its effectiveness? In order to be able to best utilise evaluations for policy-making and for strategic planning, it is important to consider the interdisciplinary aspects of evaluation and connections to other analysis techniques (e.g. Foresight Assessment). 


\section{MAPs AND GOVERNANCE ISSUES}

\subsection{Introduction - Why this topic?}

The MAP-TN deals with a special form of programme funding in RTDI policy. The specialty lies in Multi Actor settings of those funded and in a broader array of measures delivered and / or stimulated by the programme, which could also be described as a kind of umbrella for a set of public interventions. Typical examples include the Competence Centre programmes or Cluster funding schemes. Complexity is a key word: As MAPs address systemic problems like insufficient cooperation behaviour or lack of critical masses, they need to mobilise more complex actor fields and a differentiated set of measures. The other way round, as MAPs want to bring together actors from different fields, the level of complexity of the programme design, management and evaluation is much higher for all actors involved and the institutional setting as a whole, including the responsible funding agency, than in "normal" funding schemes addressing only one (type of) actor. Most MAPs have both a systemic approach and an ambition to change some of the rules and mechanisms on the systems level.

In doing so, MAP planners and managers need to take a look at the policy mechanisms and to define where, how and why they want to come forward with their interventions, whom they affect and how learning, feedback or "inhibiting" loops look like ${ }^{32}$. The picture of the policy cycle is key, though sometimes nearly as arty, ramified and transient as a Buddhist mandala. The planning and management procedures of MAPs are closely connected with a whole number of policy questions and issues of impacts on the policy level(s). So MAPs- much less than many other programmes - cannot ignore issues of governance. This includes both formal / legal and informal / cultural settings and interlinks.

In the MAP-TN we need to see and analyse on the one hand the legal relationships like general legal framework, RTDI-specific laws; funding rules and regulations or agencification and funding contracts. On the other hand the same is true for softer rules, habits and culture like traditions and history; the shape of organisations, common beliefs, taboos and shared expectations. This is of importance for more than one reason:

- Specific for MAPs is the aim to bridge the gap between at least two "worlds" - like science and industry - in the national or other given innovation system (NIS) with a high probability of different rules and routines, incentives and expectations.

32 Most planners and managers of "normal" funding programmes also need to be aware of some systemic and framework conditions of their work. But it is much easier: A science funding council asks for quality of applicants and projects and not much more. Of course this can have tremendous long term impacts on the scientific institutions where the researchers work - and the working conditions and incentives in these institutions somehow influence the way and success of the funding scheme. Nevertheless it is a much simpler thing to plan and manage a one actor / one measure scheme where only limited goals within one actor / one project are addressed. Note that the lines between MAPs and all other RTDI funding programmes are of course blurred. 
- Funding for MAPs usually originates from public sources, and is disbursed to the MAP manager via public sector mechanisms. Assuming that at least some of the MAP target groups are private organisations, this means that MAPs usually have to bridge the gap between the two "worlds" and "institutional norms" of the public and private sector ${ }^{33}$. Though this holds true not only for MAPs, it adds to their typical complexity.

This short contribution somehow repeats and modulates the analytical and descriptive parts from a different angle, focusing on the aspects of "governance" and "social norms". In the following chapter 8.2 we try to find some common ground in the very broad and soft discussion of governance and social norms, while chapter 8.3 tries to argue that MAPs can be seen primarily as a governance issue. Chapter 8.4 establishes five different levels of governance as a tool for analysing actor sets and relations, while chapter 8.5 tries to formulate some minimum MAP requirements from a governance or institutional setting perspective. In chapter 8.6 we try to give empirical examples from the MAP-TN's work, exemplifying governance issues concerning selection procedures and indicating the influences of "social norms" on the development and management of MAPs. These can be used as broad hints when being confronted with the challenge to develop or adapt (elements of) MAPs for different institutional contexts.

\subsection{The terms „governance“ and "social norms": difficult approaches}

Discussing governance issues is a slippery ground. Many different meanings and definitions are existing, the whole topic being a very soft one - both in wording and in concept. Many authors use this term to describe a "government plus ..." or „legal framework plus ..." approach, one that is useful for our concern. But: What is our concern? We need some framework where we can put MAPs into, both on a conceptual and a practical level. The number of public support organisations, research performers, firms and other organisations affected by a MAP is high; and the MAP itself is shaped by very many factors: legal and cultural, written and unwritten, global and specified. So governance is not about pure top-down, command and control, simple and clear cut approaches and relations, but "about the handling of complexity and the management of dynamic flows. It is fundamentally about interdependence, linkages, networks, partnerships, co-evolution and mutual adjustment" (De la Mothe, 2001, 3).

Governance can be described as "the effort of human communities to try to control, direct, shape, or regulate certain kinds of activities. ... (It) usually constitutes a subset of public policy more broadly.” (Fukuyama / Wagner, 2001,198). We talk about a „... broader notion than government (i.e. legal system) ... involves interaction between those formal institutions and

${ }^{33}$ This bridging function is not only true for institutional norms between private and public sector, but also between different norms concerning the logic of action: developing and carrying out a project follows a different logic than e.g. developing policy strategies and planning in election terms. Also, annual budgeting is usually not easily compatible with funding of multi-annual projects etc. We even do not talk about different institutional subsets of norms and traditions within the public sector! 
those of civil society ... no automatic normative connotation" (Int. Institute of Administrative Sciences, homepage, 1996). This broader approach is quite naturally a soft one and it is about:

- Perspectives, where conventional boundaries between politics and administration are perhaps less significant;

- the question how whole (organisational / institutional) ensembles „dance together“, with a focus on the interplay of actors;

- larger sets of actors to influence strategic, managerial and allocative agendas;

- the handling of complexity and management of dynamic flows ${ }^{34}$.

This approach is seen as a suitable way to analyse systemic policy fields like RTDI policy. It can be defined as a set of arrangements and regulations in fields where not only direct, one-way steering and control mechanisms work. Governance is a broad set of hard and soft rules including feedback loops (see also Kuhlmann 1998, referring to Mayntz / Scharpf). Similar meanings and definitions are described by Mayntz, from "politische Steuerung" via cooperative modes to analysing network economies and relations (Mayntz 1998, $1 \mathrm{ff}$ ).

As MAP-TN is a funded European Framework Programme project it is also important to note the somehow different meaning of the term governance in the European institutional debate: In this context governance often focuses on the discussion about "good“ European politics and policies in the light of public acceptance. Governance initiatives shall guarantee smoother policy procedures and less distance between European institutions and the citizens. The general definition of the European White Paper on governance is nevertheless useful for our work: „Governance means rules, processes and behaviour that affect the way in which powers are exercised at European level, particularly as regards openness, participation, accountability, effectiveness and coherence ${ }^{35 " \prime}$ (European Commission, see also Boekholt / Arnold, 2002, 4). The governance discussion is a fruitful instrument also to analyse the interplay of different actor sets in the European and National RTDI policies developing ERA scenarios (Kuhlmann / Edler 2003, Caracostas 2003)

\footnotetext{
${ }^{34}$ For the bullet points: different sources (see Boekholt / Arnold, 2002, p.3)

${ }^{35}$ Note that this definition of the term governance includes two aspects: the "active aspect" of power and the "passive" aspect of "rules affecting the way power is exercised".
} 
Finally, some authors see in the STI Policy context governance as a counterpart to government and concentrate on negotiations and incentives. Nevertheless the governance instruments for these authors are also priority setting, funding, evaluations, internationalisation moves etc. (Feron / Crowley 2002).

So governance in our context includes:

(i) The power / ability to design, influence, manage and reshape a MAP, in a broader sense also to influence the behaviour of those touched / funded by the programme. This includes indirect influence via feed-backs and learning and takes place (as we will see in chapter 8.4) on a number of different levels. Governance issues therefore are not so much about activities of one single actor, but about relationships between a number of actors. These relations are shaped and expressed by a multitude of instruments also within a MAP, from "hard" legislative or contractual measures to "soft“ facts and measures like a common history, beliefs, advice, management and cooperation support. (ii) Our pragmatic governance definition also includes a broad range of external factors and framework conditions that also influence and shape MAPs. The difference is that these general laws, habits, worldviews and trajectories are not as directly attributable to a specific actor or level than the above mentioned influencing factors.

The main interest of the members of MAP-TN, being MAP managing agents or closely linked to MAP management is to be seen in the different approaches and opportunities to actively influence MAPs (see the above mentioned approach of Fukuyama / Wagner, 198, to control, direct, shape and regulate). However, the framework of softer rules and norms shaping those opportunities have considerable effects on the design and implementation of MAPs and therefore have to be taken into account. A fruitful approach to describe and analyse these elements of our very broad governance definition is the notion of "institutional settings" and the "rules of the game".

According to North (1992), the term "institutions" means the "rules of the game", the rules and restrictions for our behaviour that guide interaction between the players ${ }^{36}$. Such rules of the game can be defined by laws, they can be a result of traditions and culture: underlying world views, values, attitudes etc. This implies that the rules of the game are partly formed by prior experience: History matters, e.g. the experience in relationships with others in general, in participating in other programmes. They define which opportunities of action are perceived and realised by the actors for themselves. And they form expectations concerning the actions of others and have therefore considerable impact on decisions ${ }^{37}$.

36 North differentiates between organisations and institutions. Organisations are "the players" (i.e. groups of individuals that aim together at a certain defined purpose), institutions are the "rules of the game".

37 Institutional economics considers e.g. transaction costs, dependencies, trust and uncertainty and the importance of expectations concerning the decisions of others. It develops explanations why organisations evolve and shows the influences of institutional norms on transactions or cooperation between those. Since MAPs by definition have a broader set of measures than financial incentives but aim at bringing together actors from different fields, and also at "changing research culture", these influences should be taken into account explicitly. Approaches based on Coase and 
Some authors even emphasise that when taking the view of institutions as "regularities of behaviour understandable in terms of rules, norms, and routines", it is inappropriate to conceive of certain decisions as deliberate choices from a given and well known set of opportunities; but rather that most of the choices are accomplished more or less automatically by "built in" routines (see Scott, 2001, 32).

For the MAP-TN the term "social norms" is used for the "rules of the game", including formal norms, but with a focus on softer rules often described as "funding culture" etc ${ }^{38}$. Hence, social norms are included in a broader notion of governance, providing a soft but powerful influence by creating and limiting opportunities.

\subsection{MAPs are governance issues and instruments}

As the term governance in our context gives us better understanding of hard and soft factors shaping social settings and realities and as it can structure like choreography the "dancing together of whole ensembles", Multi Actor - Multi Measure funding programmes seem to be at the core of the governance issue. MAPs affect many layers, procedures and institutions - and vice versa. As an example this can be illustrated with Competence Centre programmes, a typical subgroup of MAPs:

A Competence Centre (CC) tries to bring together a number of scientific research groups (sometimes at more than one institution) and a number of innovating firms. The framework is on the one hand a funding scheme, on the other hand a complex research programme consisting of dozens of individual projects. To make a CC work, a large set of worldviews and conditions of three different worlds must be streamlined: Public administration, industry and academic research. Such framework conditions include IPR, human resource management, research planning horizons and mechanisms, cooperation behaviours and incentives, leadership styles etc. A larger CC involves in different forms over seven years at least a ministry, a specialised funding agency and perhaps other funding organisations, regional actors, about five research groups from more than one academic organisation, perhaps a contract research organisation, a dozen firms of all sizes, consultants, public brokers etc. The centre management has to take account of different trajectories and styles and has to forge a distinct working style of the centre itself. There are general funding laws, CC guidelines, dozens of contracts, a general agreement and different sets of beliefs, incentives and interests ${ }^{39}$.

History clearly matters, at least in two ways. Historical trajectories shaped the norms and organisations that are relevant for and participating in MAPs. These historical trajectories -

institutional economic thought are suited to provide answers for these specific requirements (see also Coase, 1960/1988).

38 To avoid confusion in the discussion, since in our daily language use "institutions" generally means "organisations" (see also North 1992).

39 By the way it would be interesting to analyze deeper if the funding money really is the single most important governance instrument for the funding agency and the centre management. 
which can be of a planned or unplanned nature - are laws, organisation principles, quality and evaluation standards, specialisations, cooperation patterns, funding culture and routines and many more ${ }^{40}$. On the other hand MAPs are an attempt to answer, to influence, to change or to reverse these historical developments. Perhaps this is even part of the definition of MAPs: They are generally an effort to deliberately change not only the situation of one actor or to totally comply with his logic of doing things, but an attempt to change this logic and to push forward new architectures and combinations.

So MAPs strongly affect actors within complex organisational settings, and they are often designed to change agendas of large, often traditional organisations, namely in the academic and the public research sector. Competence Centres, Cluster programmes and other MAPs, besides their function to push research, foster human resource development, get hold of more intellectual property rights etc., generally want to change organisations and institutional settings. This is a typical "governance feature" of MAPs: The change agenda comes as an incentive for organisations. This means no direct change of statutes, association articles and constitutions but using programmes as a trigger for organisations to adapt. In an ideal world RTDI policy makers can combine MAPs (or other programmes) with organisational reform, while in bureaucratic reality such attempts prove to be difficult.

MAPs themselves can be seen as governance instruments on and for organisations in a given innovation system, acting as a "Glue" a "Solvent" or as a "Bypass" (see chapter 4.2.3 on roles of MAPs)

The rationale for policy makers to run MAPs is to "change research culture" without setting up new organisations. The good thing is that this approach is quicker and cheaper than building new research organisations. Most of the public RTDI budgets are full of basic funding commitments with no end date and room for manoeuvre (and reform) is tight. On the other hand MAP-funded initiatives, namely centres, face the challenge of a foreseeable ending.

To sum up, policy makers design MAPs to address governance issues. Actors running MAPs see and must see the shadows of history, the institutional settings, the hidden interests etc. They must be able to calculate trajectories and to work in interplay with a multitude of actors ... always with a change agenda in mind. The actors affected / funded by MAPs face strategic challenges and mirror signals to the levels „above“.

${ }^{40}$ For MAPs and for whole innovation systems the influencing and shaping factors are manifold. A number of other policies are strongly interrelated to RTDI policy and to include fields like labour market or education policies makes the analysis of innovation systems more fruitful, e.g. for Denmark see Lundvall (2002). On the other hand here is a danger both for RTDI policy making and programme, i.e. MAP, planning: Some policy fields cannot be reached or changed and the programmes have to adapt and compromise. Some funding programmes even failed because of detrimental framework conditions, like innovation inhibiting sector regulation. 


\subsection{Governance on different levels ${ }^{41}$}

To analyse MAPs with a governance approach, five levels are of interest. All of them show "hard" legal and "soft" cultural properties ("social norms").

- First level: General laws, government practices, cultural issues within and above a given innovation system;

- Second level: RTDI programme specific laws, regulations and practices, the given culture of programme design;

- Third level: Contracts and relations between policy making institutions like ministries and operative funding agencies;

- Fourth level: Contracts and relations between operative funding agencies and clients;

- Fifth level: Contracts, rules and cooperation culture within large consortia in different fields.

With this tool we can better describe influences, framework conditions and interdependencies between MAPs, institutional settings and regulatory frameworks (Stampfer 2003b) and we can try to find out some "minimum MAP requirements" (chapter 8.5 ) that should be in place when a MAP is designed and managed. On the level of the MAP-TN it can serve as a kind of summing up for the whole RoadMAP and its recommendations along the policy cycle.

The first two levels influence all kinds of RTDI policy activities and are open to MAP feedback only in a limited, long-term and indirect form. The third, fourth and fifth levels show many specific features in MAPs and the interaction between the programme and the surrounding actor sets is direct, constant and very strong. Note that the following attributes like abstract, farreaching etc. are only indicative and not normatively defined in any form.

\subsubsection{First level governance}

A first level of governance is constituted by very general (legal) frameworks, rules and complemented by elements of the institutional setting and social norms such as cultural habits, partly within the RTDI context, partly outside. This is often the strongest form of governance, shaping the expectations and lifes of the actors fundamentally.

An example of an RTDI context-specific framework governance are the laws regulating the organisation of universities, the incentives for cooperation, be it with other academic partners or with industry and the related cultural issues: What is success? How do career paths look like? Are we open to foreign competition? ... etc. A MAP tackling cooperation weaknesses has to analyse carefully the hard and soft factors in advance.

\footnotetext{
41 The term levels should not be understood in a strict hierarchical sense, but we speak about magnitudes and generalization / specialization. This term should not imply that one level is more important than another - taking into account a systemic view of a policy cycle
} 
Another is the issue of the set of actors and the related social norms: How many ministries are responsible for what? Is there a strong strategic advisory body? How is co-ordination managed? ... etc. (For details see Boekholt / Arnold et. al. 2002, Hart 2001.

An example of non-specific framework governance is corporate taxation, influencing firms' investment and RTDI behaviour, again no matter if they take part in a specific MAP or if they are more generally subject to public state aid. A soft issue is the innovation culture in private industry, where differences between countries, firm sizes or industrial sectors can be identified.

Finally a mixed example is European state aid policy, originating in Single Market policy and not in RTDI policy, but providing strong and very detailed legal framework as to when, how and to what extent firms performing RTDI can be subsidised by public funds.

This first level strongly shapes the actor settings and the arenas for RTDI policy makers and therefore also for MAP-makers and -managers. Governance on this level is generally abstract and not tailored to the specific needs of a MAP or even our policy field, far-reaching, longlasting and often giving unclear or contradictory signals to the actors in a MAP.

\subsubsection{Second level governance}

A second level comprises legal and social regulations establishing and determining RTDI funding programmes. Nearly all such programmes are based both on specific traditions and legal acts, the latter, in the national context, mostly designed or issued by a responsible ministry. Some countries rely more strongly on basic funding for research performers, while others come forward with dozens of specified programmes; some others relying more on strictly bottom up - single project funding mechanisms.

Funding programmes are also results of different organisational settings. Some countries have strong and independent agencies where programmes are designed and managed out of one hand. Streamlining, organised learning ${ }^{42}$ and portfolio approaches can be the positive effects of such architecture. Other countries have more or less co-ordinated, even feuding actors with programme responsibility in the RTDI scene, with different agendas, cultures and quality approaches.

Typical examples for second level governance include formal funding guidelines for a given programme or a set of programmes. Features with a strong governance impact can be the degree of strictness of guidelines, the way how winners are selected (competitive vs. non competitive open approaches), the degree of management accountability for the funding agency, the openness to change over a given time period, the possibility for political interference on the operative level etc. Note that there is always a corresponding "soft" funding culture. A good example for their influence is the difference that can be observed between the ex ante evaluation of proposals between Sweden and Austria:

\footnotetext{
${ }^{42}$ Learning from abroad, in our case from other MAPs, needs to be a highly structured process, taking into account the most important governance issues of the programme you want to learn from (Rose 2001).
} 
While in both cases the process of evaluation, i.e. public calls, evaluation by international external experts / peers according to programme guidelines etc. shows similarities, considerable differences can be observed in terms of confidentiality vs. publicity of the process and the evaluation report.

These rules are often formal as a MAP is mostly based on written and binding directives or guidelines. They are less abstract than documents on the first governance level, but still not tailored for individual initiatives. They are mostly flexible and based on knowledge about the relevant instruments and actors. Governance is still abstract, but mid-term oriented and aimed at specified actor groups. The rules are primarily binding for public administration actors who then, on this basis, exercise fourth level governance.

\subsubsection{Third level governance}

A third level concerns contracts between policy-makers like ministries and operative funding units like specialised agencies, and the culture of agencification. Again this is an issue of organisational setting (and social norms): A considerable number of programmes, including most MAPs, are run by actors other than those making the overall or specific (legal) rules and there are good reasons to separate strategic from operative business.

Here the most important instruments are contracts between the ministries and the funding agencies. In some countries there is a long standing tradition of contracting out on the basis of a number of performance criteria, while other countries still experiment with different approaches. On this level important features are the terms of reference, success criteria, procedures and other issues of a programme. On the other hand social norms like trust, habits and the overall architecture of the system matter: Are there long-standing relations between the contracting ministry and specialised agencies? Are there common worldviews? Have the programme managers evolved rather in the public sector in the form of agencies or public funds / research councils or is there a kind of a market including private programme managers? Even within the public sector, traditions and organisational form of the actors are wildly different; autonomy and flexibility are subject to tradition, to the degree of formal independence and to management features. Even within one big agency most different sub-cultures can evolve and survive, as the Norwegian RCN example shows.

Third-level governance is more detailed and still mid-term oriented. It is supposed to pre-shape the relation between an agency and its "customers“, i.e. firms and research groups being supported under an RTDI funding programme. 


\subsubsection{Fourth level („operative“) governance}

The relation between the agency and its "customers" - or in some cases the ministry / or the European Commission etc. and its "customers" - is regulated on a fourth level. This is the interface between the public actor and those funded. Note that with MAPs public actors need an especially clear agenda and interface, as they want to serve complex actor settings like science - industry consortia.

Both contractual and soft relations are designed and regulated in detail here. The culture of RTDI funding programmes mainly derives from the activities of the agencies responsible. This is the place where a lot of reality is created, a reality of course less stable than - let's say - an RTDI funding law (see first level governance). Nevertheless it is one that immediately spreads and grows. There is a strong interplay between the funding body and those funded. Learning and adaptation is a hot issue on both sides as the gluing / dissolving / bypassing / strengthening procedures evolve.

Governance is concrete and direct, feedback loops are generally immediate and strong. There are concrete rules, partly for the whole programme, partly on a case-by-case basis. The target group is clear and known. Those funded are strongly influenced both by the written rules and by the management style of the funding actor. The degree of invasiveness can vary wildly between MAPs but a number of common features include strong support of applicants, strong ex ante evaluations, multi-party contracts and managerial / financial monitoring, without disturbing the evolvement of the individual consortiums' research programme.

The degree of freedom for an agency in it's relation to customers depends on $2^{\text {nd }}$ and $3^{\text {rd }}$ level governance. This issue is crucial for learning and learning cycles as strong symbiotic relationships between agencies and those funded can result in looser ties between agency and the responsible ministry. There is in some countries an ongoing discussion about fully developed learning cycles (where you also need ministries and policymakers equipped with ears) but also about a danger of free-roving agencies not listening any longer to ministerial priority setting. Needless to say we see in some countries explicit and clear solutions, at least for some time.

\subsubsection{Fifth level governance}

The relations within large consortia become more important as the typical consortium has been growing. This happens with the new instruments in the $6^{\text {th }}$ EU Framework Programme, where subsidiarity of consortia management and of some selection procedures was - together with the wish to have larger consortia - one of the reasons for creating Networks of Excellence (NoE) and Integrated Projects (IP) as funding mechanisms. MAPs are often similar to these large consortia, some MAPs can even be seen as a kind of forerunners of NoE or IP.

Large consortia involve a big number of actors, mostly from different fields. We find a delicate balance between inner regulations and practices pre-structured by the programme and those established by the consortium itself. 
Typical issues include management, monitoring, evaluation and reporting responsibilities, the question how much of the funding money can be distributed within the consortium without being earmarked in detail by the funding agency, the possibility of partners to leave or new partners to join a consortium during a funding period, etc. There is a certain trend towards more liberalism and goal / key data-oriented programme management within agencies: Note the discussion about sub-calls issued by the funded consortia in FP6 IP's.

Governance is very direct and often linked to individual projects, deliverables, resources or results. While for research performers this means new management roles and mechanisms, the funding agencies sometimes have to learn how to deal with such a new balance. If they abstain from too many interventions they have time and opportunity to monitor the right things if the third and fourth level governance mechanisms are in place and well planned, sending the right incentive signals to the funded consortia, centres, clusters etc.

\subsection{Minimum MAP requirements}

With MAPs we face long term mutual learning processes where the most significant outputs are probably the most intangible ones. Again: one typical MAP consortium consists of a (large) variety of actors, often coming from different fields. Inside a MAP the portfolio of different consortia (centres / cluster initiatives etc.) can vary strongly, as most of the MAPs favour thematic or even organisational diversity. As MAPs are mostly large programmes spending considerable amounts of public money and as they always want to bring change to the innovation system, they are visible and attract high attention of ministers, RTDI advisory bodies, policy makers and mandarins of the involved sub-systems. They all want their say and they all have their expectations. This factor raises the challenge for strategic positioning, constant learning, but also for marketing a MAP. Some of the necessary overall requirements for MAP management in a governance perspective include the following:

First a thorough actor setting (or system) analysis before starting the programme, including a consideration of the main social norms. MAPs want to influence whole sub-systems and those responsible have to take into account the large number of influencing factors. Namely the definition of programme goals, the incentives for those funded and the management and evaluation procedures have to be designed hand in hand with a close analysis of framework conditions, namely of first and second level governance issues.

There is a need for clear roles in policy cycle, a clear division of labour regarding strategy, programme management and project level. Again, because of complexity of MAPs and the high number of those involved from different constituencies, MAPs are to be planned and handled more carefully than other rather simple programmes. The question of clear roles means also: "Do we have the right agency for such (future) programmes?"

One relevant issue in this respect are constant organised learning exercises. Adapting complex funding programmes can be a highly painful process - on the other hand a process even more necessary than in simple standard funding programmes. 
Other points that matter are:

- Clear interfaces and - even more important - a clear rationale and "message" of the MAP. There is a trade-off between clear-cut programme marketing and different target groups, nevertheless this is one decisive success factor;

- Competitive and transparent selection procedures;

- Balanced management approaches, with a good mix of determination and laissez faire;

- Special monitoring requirements to safeguard a broad array of feedbacks beyond simplistic signals and indicators;

- Systemic evaluation approaches to capture behavioural additionality, network effects, impact on level of consortium partner etc.

So we could draw a MAP governance matrix, where some minimum requirements for successful MAPs are included.

The matrix combines the five levels of governance with the five big steps in the MAP policy cycle, which is also the underlying structure of the MAP-TN. It asks for favourable framework conditions $\left(1^{\text {st }}\right.$ and $2^{\text {nd }}$ level governance, i.e. the levels rather influencing MAP agencies and stakeholders) and for suitable programme specifics and procedures $\left(3^{\text {rd }}, 4^{\text {th }}\right.$ and $5^{\text {th }}$ level, i.e. the levels rather influenced by MAP agencies and stakeholders).

This matrix can be useful for the analysis and comparison of individual MAPs. Of course some of the requirements are equally necessary for other forms of funding programmes and of course there are country and MAP specific differences. A final remark: Good practice, but not best practice is feasible (Rose 1993). 


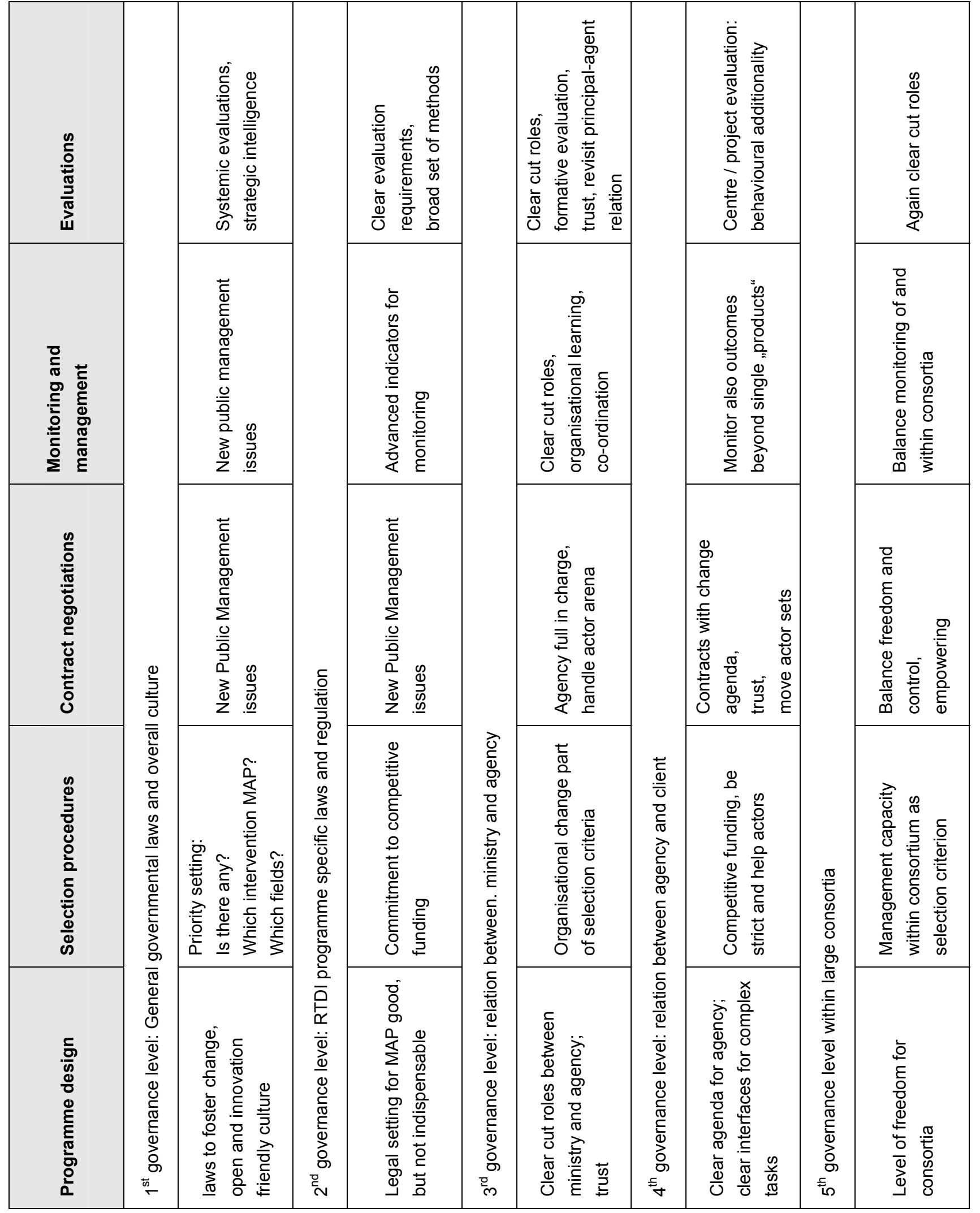




\subsection{Empirical findings from the MAP TN}

\subsubsection{Governance and selection procedures}

In this chapter we want to exemplify some governance issues concerning selection procedures. The arrangements and regulations by which MAPs may be governed do reveal themselves at this critical point in the programme lifecycle very clearly, mainly covering the range from $2^{\text {nd }}$ to $4^{\text {th }}$ level of governance in different ways

There is no particular pattern evident with respect to the overall structure of the management of MAP selection procedures, with the network comprising examples of all of the main modes of organisation. There are MAPs where the selection procedures are coordinated by a team within the Ministry with overall policy responsibility or a by dedicated funding agency or by an external contractor working to a specification for a fixed term and indeed, hybrids thereof. Only a few members of the MAP-TN manage programmes that are steered directly by ministry officials (e.g. $A U)$. Those few who have a strong role in the process use partly external management capabilities $(\mathrm{HU})$. More often ministries delegate aspects of programme management to consultants and other non-governmental organisations (DE, SL, UK, ES), leaving some of the operative authority with the ministry structures. In several cases, funding agencies are entrusted with the programme management (SE, AT, EE, NO, NL, CA, FI, BE); the Swedish agency VINNOVA, the Finnish TEKES and the Flemish IWT are examples for complete agencification of tasks.

\section{Box 29}

\section{The Estonian Competence Centre Programme: Evolving in the MAP mirror.}

Estonia takes part in the MAP-TN as observer and develops its CC programme just in time. A very small and open newcomer to the EU, Estonia embraced an agency model with clear cut roles and responsibilities early on. As in most countries there are two ministries responsible for RTD (education and economy), the CC programme coming from the Ministry of Economic Affairs (MoEA). EAS / ESTAG is the responsible operative agency. A strong RTD council designs multi-annual RTD policies, while the Ministry of Finance has a say in all budgetary planning issues and acts as regarding compliance to EU rules. MoEA was responsible for the planning of the programme, using foreign MAPs as role models and employing foreign consultants for the design process.

EAS / ESTAG was strongly involved in this design phase and will run and finance the programme after getting the necessary budgetary allocations. With the strong inclusion of foreign peers to review the proposals, the agency will be responsible for the whole selection process. The funding decision will be taken by the EAS board. There is a number of expert groups for consulting and co-ordination as well as foreign experts to monitor and accompany the policy process. Note that the general idea for the Estonia CC Programme, the set of rules and the dynamism came mainly from the ministry. The programme was just evolving and got impulses for the design of the selection procedure directly out of the MAP-TN. 
The final decision on which projects to fund often remains with the ministries (AT, HU, DE, ES, $\mathrm{SL}, \mathrm{AU}$ ). In other cases (SE, FI, NL, NO, EE, BE) the agency has the decision power. In the Canadian case, the NCE Steering Committee, consisting of a minister and presidents of the Research Councils, makes the final judgement. Note that in nearly all countries the formal decisions normally follow the expert / agency advice. In some MAPs this happened from the beginning on, while in UK the accordance rate rose due to learning and trust-building procedures.

Going down one step, some programmes sport a division of labour within the MAP management structure. One element is external peer review, other differences regard shared responsibilities for different parts of the selection procedures. In the Austrian case, the responsible funding agency (TIG) oversees and organises the whole selection procedure, while using the Austrian Science Fund to choose the scientific peers. Furthermore a specialised industrial funding organisation, the ERP Fund, has the task to review industrial and managerial matters.

Many agencies use special advisory bodies and mixed panels for the peer process (e.g. HU, SE, DE, BE, NL, FR, AU, CA). The level of complexity of such structures seems to reflect both the complexity of the MAP itself and the historically grown architecture and competencies of the actors involved. How to deal with this particular topic is associated with $3^{\text {rd }}$ level governance.

There is an equal degree of variability with respect to horizontal coordination as is the case with vertical integration between policy, administrative and performing levels. The coordination with other policy areas is an issue where all MAPs find themselves in a trade-off between necessities on the one hand and pitfalls on the other hand. This holds true for a general political level ( $1^{\text {st }}$ level governance) where intense co-ordination is sometimes seen as a value in itself and as a vehicle to blur clear responsibilities. This also holds true for the level of inter-agency cooperation ( $2^{\text {nd }}$ and $3^{\text {rd }}$ level governance), which can only succeed if there are clear roles and architectures. If such a structure is not there it is better to abstain from excessive cooperation even if others lure with extra funds. This was evident in the Swedish case, where there was a political pressure for NUTEK, the managing agency, to co-ordinate the process with another newly established Foundation. As it became apparent that the Foundation was not able to take quick enough decisions and NUTEK would probably have lost its momentum if it had opted for a cooperative call, NUTEK started the programme alone on it's own terms.

There was a presumption that MAPs as larger, nationally important initiatives, might have been expected to devote attention to various co-ordination activities to ensure harmony with other policy fields and programmes. The selection process is one possible point in a programme's life where these linkages can be explored and tested. The MAP-TN data do not bare this out. Indeed, most of our MAPs do very little with respect to policy/programme coordination and where this does happen it tends to be conducted through the interpersonal networking of administrators outside of the formal evaluation process. The UK and HU programmes do use some formal mechanisms, such as involving stakeholders and other policy interests in the evaluation process. 
The co-ordination issue is tackled in some small measure through the expertise of the evaluators when they apply the evaluation criterion relating to the additionality of the proposal i.e. that it promises a novel, original, or additional contribution to the status of knowledge. MAPs are already highly complex animals and there is a certain danger of over-coordination. In addition to that in small countries you can observe that a small number of people are sitting in all committees.

Co-ordination is nevertheless a hot issue for MAPs tackling complex actor settings with a number of measures. Cluster-like programmes must have a vital interest in links to other policy actors and measures (UK, SL), while Competence Centre programmes need much coordination efforts when the own funding resources are scarce (DE). Among the described MAPs, there are some attempts to fund selected projects in collaboration between multiple agencies (FR, FI). In the French case this inter-agency approach, seen as highly important, is to some extent hindered by the difficult administrative arrangements.

\subsubsection{Influences of formal and informal norms}

In general, the above mentioned governance issues concerning selection procedures in MAPs are about the power / ability to design, influence, manage, and reshape (parts) of these procedures in a deliberate way (see definition in chapter 8.2). But as was mentioned earlier, governance in our meaning also includes a broader range of external factors and framework conditions, including formal and informal rules (the latter we called "social norms").

In general, those norms cannot easily and deliberately be influenced; rather, most actors are not even aware of them. However, during the work in our MAP-TN we found several hints indicating the influence of social norms on particular elements in the design and management of MAPs. E.g. concerning international influences (as an example learning from good practices, integrating international experts in the design phase) it must be emphasised that it is essential to take into account the importance of the framework conditions: As best practice approaches grew highly popular namely in the OECD and EU context and it became fashionable to import foreign programme concepts, there is a certain danger to underestimate different legal, organisational, cultural frameworks and questions like budgets, size, past success etc. (see chapter 4 on design of MAPs).

For the development of good practices, i.e. of elements of MAPs that can be transferred, knowledge about governance structures including social norms is crucial. Moreover, to make use of this tool box of good examples, a basic understanding in which case to use which tool is needed.

What questions have to be asked and what knowledge has to be gained to have the opportunity to learn from each other's different MAPs, to understand their specific situation and put it in context, and to transfer elements of MAPs?

- What are relevant elements of the "action set" (see Alexander 1995, i.e. organisations interacting in the particular context-Who are the players)? This question is already dealt with in chapter 8.4 where different levels of governance were introduced. 
- What are the most relevant legal and social norms?

- What influence do they exert on the particular MAPs in consideration?

In general it can be stated that experiences of the different actors (organisations) matter in certain ways: (i) The developments in innovation processes/in innovation systems are path dependent; changes of the paths can be costly and must usually be introduced from outside the system. (ii) Experiences shape worldviews and create expectations also concerning the range of possible actions of others, be it partners, competing organisations or else. Deviations of which can hamper cooperation by decreasing trust and increasing uncertainty. Hence, new elements have to be designed and communicated deliberately and with care. (iii) These expectations and worldviews are broken down in very concrete guidelines of action. They can be laid down formally as codified knowledge, like legal requirements, guidelines, model contracts etc. or be tacit knowledge. In general, these formal and informal norms ease communication and cooperation, building trust and decrease transaction costs.

It follows that for the design and the management of MAPs not only formal rules are relevant but also informal, social norms. Concerning the choice of competitive selection procedures it turned out that in some cases there was not even a choice (e.g. UK and DE, where allocation of public funds via competitive procedures it is a requirement), whilst in some other cases competitive calls were introduced into the system as a rather new instrument.

How to find out about those informal social norms? One way would be to consider previous experiences and the expectations of the action set concerning selection procedures that would be proposers and partners, managing agency, experts, policy level. Are there experiences with participation in calls for proposals in other programmes? What are the believes concerning the typical reasoning for competitive calls what are the opportunities to learn and to change believes, e.g. by installing pilot schemes?

Examples for formal norms requiring competitive selection procedure: Especially in the UK this requirement is a matter of protocol and good practice in the UK, which has been very much part of the scientific administrator's toolkit since the publication of a seminal text back in 1971, commonly referred to as the Rothschild Report. In addition, in the UK there is a growing number of more or less official guides available to R\&D programme managers that provide advice as to which procedures to use in which situation. However, DTI officials rather draw from their practical experiences that from official guidance. On the other hand, in Austria competitive approaches for RTDI funding had no tradition (only one small programme for "Fachhochschulen" using this approach starting 1995). Hence, the reasoning for the implementation of the selection procedure of $\mathrm{K}$ plus was not only based on the motives mentioned in chapter 4 on selection procedure of MAPs, but the aim was also to initiate cultural changes in the Austrian Innovation system. Experiences showed that introducing such a new element has to be done very carefully to avoid negative side effects. $\mathrm{K}$ plus started with a pilot phase with a less sophisticated procedure than was implemented for the following calls. In Sweden, NUTEK, VINNOVA's forerunner had positive experiences with a competitive selection procedure which was used also for the CC programme. In this case, that experience, seen positively also by governmental actors, was an argument for the start of the whole programme. 
Examples for publicity vs. confidentiality: In practically all MAPs, the evaluation procedures and the criteria to evaluate against are very transparent. However, there are differences to be found. E.g. in Sweden, the reports of interim evaluations are published. In Austria, the Competence Centre gets the report; in Canada, parts of the report, leaving out some critical points, are published. Another issue is about how do the evaluators' recommendations get binding character? In Austria, they are part of a formal agreement between the funding agency TIG and the centre. In Sweden the binding character stems rather from the publicity of the report. Those cultural differences also shape the way how experts and peers are dealt with e.g. anonymous / non-anonymous evaluation. In a less transparent and more towards bilateral intervention oriented climate it is reasonable to change at least partly the evaluation group from call to call.

These few selected examples from our MAP-TN show the influence that not only formal but also informal rules / social norms, can exert on the design and management of MAPs. The lessons we learned are obvious:

- Previous experiences of the different actors have to be considered.

- Don't underestimate the influence of different legal and cultural frameworks when using good practice models.

- Use international expertise not only to learn from good practices, but also to broaden your view by trying to identify and confront different social norms.

- Introduce new elements into the system carefully, taking into account the uncertainty arising from deviations from those norms. 


\section{ORGANISATIONAL LEARNING}

\subsection{Introduction}

MAPs are programmes that address (sub-)systems of a national innovation system, trying to get whole fields moving in a certain direction and intend a behavioural and / or institutional change. The rationale behind such an intervention is to stimulate cooperation between actors, to support creation of new and exchange of existing knowledge - in other words: to increase intelligence and trigger learning processes in the system.

\section{2 "Organisational" Learning?}

Common understanding of "learning" is usually person-based: a child learns to talk, in school pupils learn to write, later on people might learn a language or acquire special skills in their professional life. The insight that also organisations have the ability to learn has become the focus of interest only in recent years. Pioneering work in the diffusion of the concept was done by Peter Senge or Chris Argyris (Senge 1990, Argyris / Schon 1978). The founding of SOL Boston (Society of Organisational Learning) and the subsequent spread of the concept through more than 30 countries worldwide ${ }^{43}$ helped disseminate the concept of Learning Organisations by establishing "communities of practice".

In MAP-TN the understanding of Organisational Learning $(\mathrm{OL})$ is that OL provides a framework for a wide range of activities and instruments that enable organisations, programmes and projects to learn and remain innovative - thus stimulate learning effects beyond the learning of individuals.

\subsection{Organisational Learning in MAP-TN}

MAPs have a level of complexity resulting from the systems approach and the Multi Actor and Multi Measure approach which - together with their long-term orientation - means that MAPs will remain effective only if they have the ability to adapt and innovate in other words: to learn.

It is common understanding that these characteristics of MAPs require programme managers to consider how learning activities can be included in the programme. Experience from MAP-TN shows that learning loops can already be considered during the design phase (DE, EE) but are more likely to be implemented later on (AT, HU, SE, SLO, UK).

The amount and type of OL activities in MAP-TN varies but most programmes do include elements, some more explicitly than others. While some MAPs are regarded as being "one big learning experience" (SE) others have designed specific elements of OL on the project level

\footnotetext{
${ }^{43}$ For details see: www.solonline.org
} 
(EE). Compared to other features of MAPs the topic of OL is much more diverse in MAP-TN programmes and a categorisation of individual approaches difficult.

Of course there is also a close connection between OL and Evaluation: an evaluation can work as an impulse to trigger learning activities in a programme, a project, an agency. The main difference between $\mathrm{OL}$ and evaluation is that the latter always contains some form of judgement. If and how this judgement works as an impulse to trigger changes in behaviour and attitudes depends on the willingness and ability of those being evaluated. What MAP managers can do to facilitate this learning process is documented in the case studies below (e.g. design of and follow-up activities after evaluations) and forms part of the MAP service pack that MAP managers tend to offer.

The following examples shall document the diversity of approaches. The presentation in the form of case studies reflects the general approach in OL literature and practice: learning is seen as highly context specific. There is no ready-made formula for enabling learning processes in organisations. Activities in OL are successful only when the organisation itself is willing to adapt and learn.

\section{Box 30}

\section{The Swedish Competence Centres: Building Leadership Potential}

The Swedish Competence Centre programme started 29 centres in one big bang. The responsible programme manager NUTEK/VINNOVA deliberately decided not to stipulate the way how to organise the centres within the universities. As a consequence the 29 centres more or less had to swim on their own and the support they received from their university managements varied a lot between participating universities.

Three international evaluation rounds have been performed so far, and especially the first one, which focussed on management issues, was systematically used to develop the centres and the programme. One of the recommendations made by the review team was to establish formal training programmes for centre leaders and annual meetings of all centre leaders should be convened with an agenda aimed at disseminating best practices, building teamworks, etc. around the centres.

As a consequence NUTEK/VINOVA started a "centre leadership project", which has strongly contributed to the organisational learning within the centres and the programme. The main theme of the project was to develop the leadership potential in network organisations. This was done through structured experiential learning in the group of centre Directors, with the support of skilled development- and process leaders. The project was built on two parallel processes: (i) A tailor-made programme was created for leadership development based on experiential learning and on the process leaders' interventions in the development process and their knowledge of leadership development. (ii) A step-bystep construction was made from the common base of knowledge through documenting the directors' experiences. Models and common conclusions are based on experiences of this kind of leadership (grounded theory).

During the course of the project several examples of models for tackling the challenges of leadership appeared examples and models which are interesting also in a broader perspective, both for other scientists leading network organisations and for universities generally. The knowledge development project reflects the development of Competence Centres in relation to changing processes going on within the universities nationally as well as globally. 
As a consequence of this project many Competence Centres have arranged follow-up activities like leadership and management training days in order to develop their organisation. It is noteworthy that all centres have changed their internal organisation one or several times. Some centres have made considerable changes after five years of activities based on the experiences gained.

\section{Box 31}

\section{The British "Partners in Innovation" - Continuous Learning Efforts with a Link to Evaluation}

Although Organisational Learning is not an explicit philosophy within the British "Partners in Innovation" programme there is a professional desire on the part of the programme management to operate a scheme that continues to please its constituents over time and one that generates sufficient visible impacts to protect the budget against the claims of competing sectors and policies. In this regard, PII has no formal OL elements. However, the scheme operates within an annual cycle and was designed from the outset to evolve in line with changing industrial and technical priorities. As such, it began with a number of learning mechanisms and has acquired more over time. These include:

A stakeholder conference to announce the broad strategy for the forthcoming round, with the opportunity for detail design inputs by the research base.

A Closing Report for each annual competition, which covers the effectiveness of the entire process and its results. This is based on an analysis of the results, an online questionnaire survey of all applicants and a survey of appraisers.

Appraisers' performance is rated by officials and this feeds forward into the register of experts maintained.

On the portfolio of projects, there are: (i) Monthly management meetings, which discuss routine management questions and ad hoc issues (e.g. the benefits and costs of the programme seeking to build better links with other construction research programmes elsewhere in Europe). (ii) Quarterly reports at the portfolio level, which report on progress with contract negotiations, the timeliness of project execution against milestones, the quality of outputs, etc. (iii) Post project impact assessments prepared by the management contractors overseeing projects are signed off by ministry officials, which feeds data into the MIS on efficiency and quality and permits.

On the level of administrators: (i) Officials have an annual appraisal system, which is linked in some small part to their performance with regard to the success of the elements of the research portfolio for which they are responsible. (ii) Management contractors have comprehensive reporting procedures and annual reviews, which link performance of the portfolio to performance of the contractor. The contracts are re-bid every three years. 


\section{Organisational Learning in Austrian K plus Centres}

Competence Centres operate under fragmented and heterogeneous organisational conditions; for example, different corporate cultures meet in such centres and project team members are sometimes scattered over a number of different locations. Classical management approaches concentrate on project management, HR development or team building with a focus on producing the required output. The question of learning of individuals and organisations are usually not in the centre of attention and therefore often remain marginalised.

In order to tackle the issue of organisational learning, the programme management agency Technologie Impulse Gesellschaft (TIG) and the Society of Organisational Learning (SOL) initiated a pilot project in 2001 in which four Competence Centres participated. The method chosen for this project was action learning. The issue of learning and innovation processes was discussed via the topics of "Person-based knowledge and fluctuation", "Developing competence in the centres" and "Fragmented corporate culture".

Each participating Competence Centre was visited by a group consisting of other centre managers, consultants from SOL Austria and TIG in order to discuss these focal topics. The aim of these visits was to conduct an open discussion of the respective centre's position in a workshop which typically lasted a whole day. In order to gain the most comprehensive possible view of the Competence Centre in question, that centre's management, area managers and project leaders, as well as staff delegated by the centre's industrial partners, participated in the discussion. On the basis of these focal topics, concrete results applying to all involved centres emerged in the key strategic areas of entrepreneurship, competence development, organisational culture, fluctuation and strategic orientation. The participants found the contact with members of other centres very useful. The exchange made them aware of challenges which they all share even though the participating centres work in different fields. In fact, it was these very differences which made it possible to examine the more general issues of organisational learning to which little attention is devoted during the centres' day-to-day business.

The results were laid down in a report that is used by TIG to stimulate awareness for aspects of OL in new $\mathrm{K}$ plus centres. Its main messages are: (i) Fluctuation is a challenge and a chance: loss of key competence is especially hard for knowledge generating centres but with new people new ideas flow in, (international) networks can be built. (ii) Build up of knowledge beyond individual RTD projects is of vital importance: internal workshops, matrix organisation, informal gatherings to disseminate information are ways to build knowledge across different projects and areas within the centre. (iii) People with different backgrounds working on a temporary basis pose a challenge for building up an identity of the centre. Strong leadership, a clear mission and visibility (physical centre, corporate identity) help to build an identity for the centre and the people working in it. (iv) Centres need the chance to emancipate from founders and owners - a good degree of entrepreneurial spirit in the management team of a centre helps to achieve that goal.

As a result of this positive feedback there was a follow-up project in 2003 with seven $\mathrm{K}$ plus centres, now focussing on a set of questions that also reflected the more mature nature of centres evolved. 
Box 33

\section{The Hungarian KKK Programme - Communication matters!}

The Hungarian activities in OL focussed on one particular aspect of OL which can be seen as a necessary precondition for learning - the ability to communicate. Centres were asked to develop in-centre training courses for personnel development focussing on management and communication.

Examples include a 4-days workshop organised by a centre on communication strategies, which resulted in significant changes in personal relations and communications channels - especially between university researchers and industrial researchers. Other centres organise weekly "scientific workshops", where students, researchers and also external people present their results and pose their problems for the others. During these seminars also management problems of the centre are discussed resulting in a deeper understanding of cooperative activities.

Activities specifically directed at the centre management include regular meetings of the directors intended to enable the exchange of experience and the establishment of "best practices" for management related topics.

\section{Box 34}

\section{The Estonian CC Programme - Learning from international experience}

The Competence Centre Programme in Estonia was launched in the beginning of 2003. The plan was to include an element of Organisational Learning already during the implementation of the programme: To allow the applicants to learn from international experience it was recommended by the programme management to involve centre directors from Austria, Sweden etc as part of the activity financed by the grant to prepare the Full Proposals. While the centres in Estonia will probably be slightly different from their counterparts in Sweden or Austria the idea was to establish a transfer of experience with those elements that will be comparable: setting up an organisation, building teams, establishing leadership in a highly innovative environment.

\section{Box 35}

\section{Mechanisms to facilitate Organisational learning in Flanders}

Organisational Learning between MAP-type platforms (i.e. VIS and SBO programmes) is stimulated in Flanders through the following coordination mechanisms :

Annual meetings for a given type of programme (e.g. VIS) are organised for project leaders and key personnel of MAP-type platforms in order to convey and exchange practical experiences and good practices;

Meetings are also held every six months to bring together the individual MAP-type platforms which are related to the same broad technology area (e.g. surface and micro/nano-technology; ICT; environmental technology);

There exists also a funding mechanism directed at universities in order to enable them to professionalise their interface offices (i.e. develop expertise in IPR strategies; contract management with industrial partners; spin-off services etc...). Representatives of the interface offices of each of the 5 Flemish Universities are brought together in a steering group managed by IWT. The objective is again to facilitate the development of good practices and exchange of experiences. These interface offices can 
indeed play an important role to support research groups to initiate and manage MAP-type platforms.

With regard to university interface offices, one criticism is often expressed that they are too far away from the actual research groups and/or too horizontal in scope or too subcritical in order to adequately understand the utilisation value of scientific developments from all fields of research. The existing culture within academic research groups also tends to focus on the research as such and not yet sufficiently on the ultimate goal of "how to contribute to move this research into a commercially viable product". A pragmatic solution has been implemented by "Leuven Research and Development", which is the interface office of the K.U. Leuven ( http://www.kuleuven.ac.be/lrd/ ). This approach involves the $50 \%$ assignment of a postdoctoral researcher from within research-intensive university departments. This person acts as an intermediate between the department and the interface office. After receiving ample training from the interface office, he or she continues to work in the department in order to provide a focussed facilitation. This allows for a more concentrated effort to better understand industry trends as well as to deepen the the specialised contact base and interactions with key industry players and to contribute to the triggering of new spin-off activities.

Of course "organisational learning" is also critically important for agencies managing MAP-type funding programmes. In this respect, main learning mechanisms for IWT-Flanders are its direct involvement in the coordinating mechanisms which have been indicated above. In addition IWT's interactions with agencies managing similar programmes in Europe (i.e. MAP, TAFTIE and possible future ERANET projects) are also highly relevant in this regard.

\section{Box 36}

\section{The Australian CRC programme - the significance of corporate memory}

During the interviews conducted for the StarMAP report Australia it turned out that a lot of information was available on current activities but it was difficult to find out about the "early days" of the programme, mainly for two reasons: (i) a central policy document describing the background and rationale for the CRC programme from the time when the programme was established (1989/1990) does not exist and (ii) due to several changes in the programme administration during the last 13 years "corporate memory" especially on the early days is not very strong. The key person during the initial phase of the CRC programme, the former Chief Scientist, Prof. Ralph Slatyer, retired some years ago and fluctuation in the $\mathrm{CRC}$ secretariat was high.

This loss of "corporate memory" clearly did not affect daily business in the programme management and selected people in the CRC system still had knowledge about the initial phase of the programme. But there was some indication that relations to individual centres were affected by frequent changes in the programme management personnel. The lesson to be drawn from this example is that especially with long-running programmes some means of storing and transferring knowledge between individuals are necessary to enable the organisation to accumulate knowledge and gain stability.

\subsection{Impulses and effects of Organisational Learning}

Despite the broad spectrum of OL activities in MAP-TN the impulse for starting activities in this field usually comes from the same source - the MAP agency (AT, GB, HU, SL). Only in two cases (SE, EE) was the original impulse an external one (evaluation, international examples) but the agencies played a central role in further developing the learning activities. 
The effects of applying instruments of Organisational Learning are perceived as highly significant in all cases. They range from better performance (SL), changed communication patterns and a better understanding of the "world outside" (HU), more systematic approaches to learning in agencies and centres e.g. "Quality Circles" in MAP agency (SE, AT) to substantial changes in the management of the programme (move from single to two-stage application, seperation of funds into strands, installation of a steering committee) (UK).

\subsection{Conclusions}

Although not always part of the MAP design Organisational Learning (OL) is now an element in most MAPs. A broad diversity of approaches exists but wherever OL is applied the results are seen as highly significant for the programme management as well as for the funded projects. The MAP managers (agencies) play an important role for the establishment of OL elements but inputs also come from external sources like evaluations or international examples. There is strong evidence that the results of OL processes enhance the stability and performance of MAPs on the programme and project level.

MAPs are in most cases managed by external programme agencies. Those organisations - as any organisation - need to learn to remain innovative in changing environments. Giving room for bottom up initiatives, the establishment of feedback loops or common reflection of findings and procedures like Quality Circles are crucial elements to make use of existing potential in those organisations.

$\mathrm{OL}$ is not the solution to every problem. But especially for MAPs it provides effective instruments to support the necessary processes of adaptation and innovation especially when it comes to changing cultures. It can also be used to deepen the understanding between programme and project levels in MAPs through joint OL activities.

Besides producing specific outputs like patents, publications, excellent people MAPs can be described as big learning exercises. Individual actors in the innovation system are stimulated by MAPs to meet and interact - to do so in a productive way they need to be open for new insights, build new relationships and structures and change their way of behaviour. To be successful they need to learn - and OL in MAPs is a way to strongly support that. 


\subsection{Lessons learned - recommendations}

Simply be aware of the fact that organisations need to learn to remain innovative. That is already the first step towards successful learning exercises.

As a programme manager consider elements of organisational learning in connection with established parts of your MAP, e.g. as a follow up activity to enable learning processes after evaluations.

Learning can result in the need for changes in the design of your programme - but such changes need to carefully consider existing practices, cultures and expectations. "Learning" must never be used as an excuse to constantly change the rules of the game!

You cannot force learning. Rely on incentives and motivation.

- Establish communities of practice and remain open minded. Have trust in the learning process whatever the outcome may be. 


\section{CONCLUDING REMARKS}

As noted already in the introduction and it should be stressed at the very end is the importance of learning: both from each other, in organisations and on an international level. What you have just read was mainly based on the exchange of experience between programme managers and this network was an attempt to learn from each other, to create and share a common view (also by drawing from the tacit knowledge developed within the MAP-TN partners by different instruments, such as benchmarking activities, staff exchange), as well as identify differences; in the roadMAP we tried to codify this knowledge.

This knowledge building which was carried out in MAP-TN, will be one of the aims of a scheme initiated by the European Commission within the 6 Framework Programme: ERA-NET. MAP-TN can be regarded as forerunner project of ERA-NET with regard to the first step of the scheme, but ERA-NET goes a step further, a step towards creating multi-national RTDI programmes. But this final step can only be achieved when having carried out a comparison between different programmes, which was done in MAP-TN and StarMAP, as well.

It has to be mentioned that applying the suggested "standards" or "good practices" also entails risks. The major risk might be to "copy \& paste" a programme without considering the respective NIS (and systemic failures), the governance system and the respective funding cultures.

Conclusion:

Applying "good international practices" is successful when national frames and conditions, cultures und research traditions are respected! 


\section{AUTHORS AND ACKNOWLEDGEMENTS}

in alphabetical order

\section{Main authors}

- Birgit Baumann (TIG, AT)

- Susanne Bührer (ISI, DE)

- Heather Greer (SEI, IE)

- Harald Hochreiter (TIG, AT)
- Sabine Mayer (TIG, AT)

- Paul Simmonds (Technopolis, UK)

- Michael Stampfer (WWTF, AT)

- Dorothea Sturn (TIG, AT)

With the support (through papers, feedback and discussions) of the MAPpers:

- Erik Arnold (Technopolis, UK)

- Peter Büttner (EARMA)

- Bernard de Potter (IWT, BE - Flanders)

- Peter Debreczeni (NKTH, HU)

- Jakob Edler (ISI, DE)

- Staffan Hakkanson (VINNOVA, SE)

- Staffan Hjorth (VINNOVA, SE)

- Rafael Ispizua (Asociación Unitec, ES)

- Stefan Kuhlmann (ISI, DE)

- Jan Larosse (IWT, BE - Flanders)

- Nina Ludewig (ISI, DE)
- Mattias Lundberg (VINNOVA, SE)

- Karin Männik (MINECO, EE)

- Alina Ostling (Technopolis, UK)

- Iztok Palcic (PMIS-lab, SL)

- Ilmar Pralla (Enterprise Estonia, EE)

- Paul Schreurs (IWT, BE - Flanders)

- Brane Semolic (PMIS-lab, SL)

- Eric Sleeckx (IWT, BE - Flanders)

- Katharina Sojde (VINNOVA, SE)

- Ben Thuriaux (Technopolis, UK)

- Borja Ullibarri (Asociación Unitec, ES)

With the support (through feedback and discussions) of the StarMAP Steering Committee:

- Claire Nauwelairs (Merit)

- Jari Romanainen (Tekes)

- Andreas Schibany (Joanneum Research)

A special thank is given to Natascha Hoskovec (TIG), responsible for all financial issues of the projects and the real but unfortunately mostly invisible "pearl" of the network, to Barbara Kunz (TIG) for all kinds of organisational and design issues and to Paraskevas Caracostas and Elie Faroult (both EC) for their support from Brussels! 


\section{ANNEX}

\subsection{Definition of RTDI funding programmes}

As it seems there is no exhaustive standard definition available, MAP-TN also developed a definition of RTDI funding programmes as indicated in chapter 2 on definitions

\subsubsection{Characteristics of RTDI funding programmes}

In the MAP project we deal with Multi Actor - Multi Measure initiatives in the form of funding programmes. Therefore the definition of MAPs has to be based on a definition of programmes as a special tool of RTDI policy ( $\rightarrow$ see definition MAPs, chapter 2 , which always have to fulfil the criteria for programmes and a number of special criteria, see also OECD 1995)

\subsubsection{What are the characteristics of an RTDI funding programme?}

1. In our context RTDI funding programmes are a form of public support of RTDI activities. Such activities are performed by the private and / or the public sector. They are not identical with „RTDI programmes“, the latter meaning not the organised support, but the organised performance of RTDI activities, e.g. as an interlinked number of projects to achieve an overarching goal.

a) It has to be stated that "funding“ must be seen in a very broad sense. While in most cases this will mean direct support for RTDI performance like grants, the definition includes also programmes funding networking activities, or providing other financial and managerial "glue" for ensuring the cooperation of different actors.

2. RTDI funding programmes provide a structural framework for the funding or other ways of support of a number of single projects or initiatives. In most cases this framework is stated in written programme guidelines or similar documents which are not subject to frequent change of rules or wide interpretations. Such guidelines are normally binding for the authority responsible for and entrusted with the programme. In this framework generally the following issues are stated:

a) Goals of the programme.

b) Responsibilities of the different actors: Who is in charge of the supporting mechanisms brought forward by the programme? $\rightarrow$ Usually a programme management unit within the authority or entrusted by the authority. Who can get the support offered by the programme? The last point is very important because many programmes address a specific target group.

c) Definition of the activities supported by the programme: In case of RTDI Funding Programmes this will in most cases be R\&D work; but also studies, transfer and dissemination issues and a broad range of other activities, namely the funding of networks are within the scope of such activities. 
d) Definition of the ways of support: RTDI funding programmes can show also a wide range of instruments, the most prominent ones being of financial nature like grants, loans or guarantees. Many programmes (also) include „softer“ measures like information, advice, business support etc.

e) Definition of an annual or multi-annual budget.

f) Definition of the duties of both sides (supporting programme management and supported initiative) regarding legal obligations, reporting, monitoring, subject to evaluations etc.

g) Possibility to evaluate the whole set of actions covered by a "programme“ in a coherent way. This means again: Are there explicit common goals? Are there explicit rules of procedure? Are there measurable outputs and impacts which can be attributed to the programme?

3. RTDI funding programmes have - as already mentioned above - within their structure a set of goals and a set of rules that should specify the activities supported by the programme and distinguish them from other activities. Such specifications can be technology-oriented (e.g. „Biotech Funding Programme“) or defined in a more horizontal way aimed at functions within the innovation system (e.g. „Programme to foster cooperation science - industry").

4. Within an RTDI funding programme the activities supported shall serve the common thematic or structural goals of the programme. The links between the supported activities under the programme umbrella (e.g. a Biotech programme) can be rather loose (,it has to be scientific Biotech projects“) or strong („the funded projects must be part of a managed thematic, interdisciplinary network").

5. RTDI funding programmes generally have a limited lifetime („the programme runs from ... to ...) or the lifetime of the programme ends when the last project or other initiatives supported by the programme ends. Projects always have a given timetable with a defined end-date.

6. An RTDI funding programme itself is different from a "legal entity“ and from an „institution“; but an instrument accessible and of possible usefulness for individual and / or legal persons.

7. An RTDI funding programme is an activity / a set of activities run and governed by a responsible authority ( $\rightarrow$ see 2 .), i.e. a RTDI funding institution like an agency, a ministry, or a research council. 


\subsubsection{Instruments different from RTDI funding programmes}

Programmes are different from generic funding activities and schemes, from organisations and from projects:

Direct generic funding schemes: Typical for this kind of RTDI funding instruments are agencies or research councils giving support (in the form of grants or in another way) to initiatives brought forward by a RTDI performer, be it a firm, a scientist or comparable natural or legal persons. This support is granted, project per project, under a set of common rules. Such rules specify only lifetime, RTD intensity, funding rates, the form of the proposal and similar formal characteristics of applicable initiatives. These direct generic funding schemes often have a limited lifetime, but there are also many cases where such a scheme is open for decades with no ending date, being the main „product“ of a funding institution. Such schemes generally neither have a thematic focus nor a strategic orientation or strategic goals. E.g.: Their mission is not to "boost Biotech applications to strengthen young Biotech firms within the next three years", but to „enhance general competitiveness of the Nation's industry“ by open single project funding. Of course the borders between such activities and RTDI funding programmes are blurred; the same is true for the borders between schemes and the funding institutions running them.

Indirect generic support activities: Instruments like RTDI tax credits are no funding programmes because there is generally no limited lifetime of this activity, there are generally no specific goals and no thematic / structural missions. Moreover, there is by definition no set of managed activities as funding instrument.

Organisations: Organisations generally do not have a limited lifetime but are considered permanent fixtures, even when they undergo periodic evaluation. The normal features of an organisation are: Long term mission; possibility to act as a legal person with all rights, duties and liabilities; written company agreement, formal boards and bodies, most of them according to law; director(s) and employees; etc. Institutions as legal persons can apply for and run / manage RTDI funding programmes and / or projects.

- Special issue: Programmes for the set-up of institutionalised RTDI performers like Competence Centres. Some programmes try to trigger cooperative R\&D by selecting and funding new institutions with a limited life-time. The main task of these institutions is to run a number of cooperative R\&D projects and to perform related activities, often in form of giving an initial boost for consortia that shall work in a self-sustaining way after the end of the funding. Nevertheless the "nature" of an RTDI funding programme is given, as long as the characteristics under 10.1 .2 are there.

- Funding Organisations: As stated under 10.1.2, a programme has to be governed / to be run by a responsible authority. These authorities are organisations, their instruments are inter alia RTDI funding programmes.

Projects: Projects have a clearly set lifetime, a work plan and include a well defined task. Whether small or large, they are targeted at one issue (....). Projects can be part of programmes, while programmes are generally an umbrella for a number of projects. 


\subsection{Competence Centre Programmes and Network oriented Programmes}

\subsubsection{AT: K plus - Competence Centre Programme}

The $\mathrm{K}$ plus Competence Centre Programme funds collaborative research facilities with a specified life time that were set up to carry out top quality, long-term and internationally compete tive research and technological development (RTD) projects at a pre-competition stage. The goal is to perform research that is highly relevant for both the academic world and industry and to develop human capital in areas that are either multi-disciplinary or which are relevant for a number of sectors / companies. Up to now $18 \mathrm{~K}$ plus Centres are established.

Contact (from MAP-TN):

Harald Hochreiter / TIG - Technologie Impulse GmbH

www.kplus.at

\subsubsection{AU: CRC - Cooperative Research Centres}

The CRC Programme's mission is to strengthen long term collaboration between research organisations, and between these organisations and the users of research, in order to obtain greater benefits from Australia's investment in R\&D”.

The objectives of the CRC Programme are to contribute to national objectives, including economic and social development, and the establishment of internationally competitive industry sectors, through supporting long term, high quality scientific and technological research;

to stimulate a broader education and training experience, particularly in graduate programs, through initiatives such as the active involvement of researchers from outside the higher education system, and to enhance the employment prospects of students through initiatives such as involvement in major cooperative, user oriented research programs;

to capture the benefits of research, and to strengthen the links between research and its commercial and other applications, by the active involvement of the users of research in the work and management of the Centres; and

to promote cooperation in research, and through it a more efficient use of resources in the national research effort by building centres of research concentration and strengthening research networks.

Contact:

Marea Fatseas / CRC Programme, Department of Education, Science and Training, Canberra www.crc.gov.au 


\subsubsection{SE: Competence Centre Programme}

The Swedish Competence Centres Programme is a long-term effort to strengthen the very important link in the Swedish innovation system between academic research groups and industrial R\&D. The programme comprises 28 Competence Centres at eight universities. It is managed by VINNOVA in collaboration with the Swedish Energy Agency, STEM, which is cofinancing five of the Centres.

Contact (from MAP-TN):

Staffan Hjorth / VINNOVA

$\underline{\text { www.vinnova.se }}$

\subsubsection{HU: KKK - Cooperative Research Centers}

KKKs main objective is to create, or to strengthen the operation of, research centres allowing the formation of integral ties between the institutions of Hungarian college and university (higher) education, other non-profit research institutions and the enterprise-business innovation sector, and wherein the strategic integration of education, research and development, knowledge and technological transfer can be realised. It is aimed at as an institutionalised form of science/industry relations, with a long-term strategy and concentrated financial support for RTDI projects. An important factor was to help (force gently) universities to articulate a welldefined research strategy of several research units of the university in co-operation.

Note: At present, the Ministry does not maintain a separate web site for the programme. However, information about the centres involved in the programme is available on the following pages:

PTE - DD-KKK: http://www.ddkkk.pte.hu/english/index.html

ME - MeAKKK: http://www.meakkk.uni-miskolc.hu/

VE - VEI-KKK: http://vikkk.vein.hu/eng/index.htm

SOTE: http://www.webio.hu/kkk/

BME: http://www.etik.hu/index-en.html

Contact (from MAP-TN):

Peter Debreczeni / Ministry of Education

www.om.hu 


\subsubsection{DE: CCN- Competence Centres for Nanotechnology}

The programme supports activities in the nanotechnology sector, especially for industrial application. The scheme has the following main objectives: (i) Optimal transfer of (nanotechnological) knowledge into marketable products, processes and services; (ii) Setting up a competence profile in the selected technology field which makes the location attractive and well-known, at the national as well as at the international level.

Additionally, the CCN shall: improve education and further training; engage in public relations activities (in order to provide public acceptance for the technology field, to attract investors etc.); create an economically attractive environment; counsel mainly industrial prospects in the corresponding field of nanotechnology; develop standardisation activities

Six competence centres for nanotechnology were granted since 1998.

Contact (from MAP-TN):

Susanne Bührer and Jakob Edler / ISI - Frauenhofer Institute for Systems and Innovation Research

www.nanonet.de

\subsubsection{IE: Energy RDD Programme}

The Energy RDD Programmes mission is to stimulate and accelerate the generation, delivery and implementation of demand side and supply side solutions contributing to a sustainable energy economy in Ireland. These solutions, leading to improved products, systems, practices and policies, are to be created by portfolios of research, development, demonstration and associated projects supported through a set of focused sectoral [R\&D] programmes". The Energy RDD Programme is operated by the IEC/SEAI.

Contact (from MAP-TN):

Heather Greer / SEI - Sustainable Energy Ireland

www.sei.ie 


\subsubsection{SL: The Toolmaker's Programme}

Slovenian industrial policy aims at promoting competitiveness with an emphasis on skill formation and industrial development by implementing two interrelated programmes, the programme to promote entrepreneurship featuring the subprogram IV cooperation, networking and cluster development and the programme to support structural adjustment and restructuring.

The Ministry's of Economic Affairs "Subprogram IV: Enterprise Cooperation and Cluster Development" aims to enhance cluster development in Slovenia through the co-financing of joint projects in the fields of product development, marketing, technology and value chain. The pilot has launched three cluster initiatives in the automotive, logistics/transport and machine tool sectors. The University of Maribor hosts the latter centre.

Contact (from MAP-TN):

Brane Semolic - University of Maribor, Faculty of Mechanical Engineering www.uni-mb.si

\subsubsection{UK: PII - Partners in Innovation}

Partners in Innovation is a collaborative scheme which provides up to half the costs of research and innovation (R\&I) projects within the construction sector. It is open to all UK companies, industry bodies, institutions, research and technology organisations and universities in the UK (England, Wales, Scotland and N.I.). PII encourages the pursuit of common goals through active partnerships between government, industry and research organisations. PII is the main mechanism for providing government support to collaborative projects for construction related research and innovation. All research proposals should be able to demonstrate relevance to the key economic, social and environmental policies set out in Rethinking construction and Building a Better Quality of Life.

Contact (from MAP-TN):

Paul Simmonds / Technopolis Ltd.

www.pii.org.uk 


\subsubsection{BE/Flanders: VIS (=CIN - Co-operative Innovation Networks)}

The aim of CIN is to regroup dispersed activities, to create awareness for (technological) innovation in industry, to create powerful networks through joining actor's forces and creating a critical mass. It is seen as seed for the new Centres of Excellence.

CIN provides subsidies for three types of activities: collective research, technological advice and/or technological innovation stimulation; in addition to that CIN coordinates activities between all of groups of the running projects. Eligible for funding are structural cooperations between (in general) Flemish companies, in case together with knowledge organisations (universities, R\&D centres, ...) and can be seen as counter part of support to individual companies.

\section{Contact (from MAP-TN)}

Bernard de Potter

www.iwt.be

\subsubsection{ES/Basque Country: PCTI Cooperación}

PCTIs mission is the improvement of the competitiveness of the Basque companies. PCTI is embedded in the Industrial Policy of the Basque Government which defined the Technology Policy to follow. This Industrial Policy has as one of its three major axis the promotion of structural change of companies towards a networked and cooperative operation, away from traditional individualistic practice.

Contact (from MAP-TN):

Borja Ullibarri / Fundación Unitec

www.euskadi.net/industria

\subsubsection{EE: Competence Centre Programme}

The mission of the Estonian Competence Centre Programme is to integrate the Estonian RD\&l potential through the establishment of the new structural initiatives in the Estonian Innovation System in a longer-term perspective. This is one of the ambitious plans that the Estonian R\&D strategy „Knowledge-based Estonia“ has stated for 2002-2006. It supports the vision of the strategy that is: „Estonia is a knowledge-based society where new knowledge, the application of knowledge and skills, as well as the development of human capital, constitute the source of economic and labour force competitiveness and an enhanced quality of life“.

Contact (from MAP-TN):

Ilmar Pralla / Ministry of Economic Affairs and Communications

www.mkm.ee 


\subsubsection{CA: NCE - Networks of Centres of Excellence program}

The goal of the NCE Program is to mobilise Canada's research talent in the academic, private and public sectors and apply it to the task of developing the economy and improving the quality of life of Canadians. This goal is consistent with, and reinforces, the three pillars of the federal Science and Technology Strategy: sustainable job creation and economic growth; improved quality of life; and, advancement of knowledge.

The NCE program invests in national research networks that will:

stimulate internationally competitive, leading-edge fundamental and applied research in areas critical to Canadian economic and social development;

develop and retain world-class researchers in areas essential to Canada's productivity and economic growth;

create nation-wide multidisciplinary and multi-sectoral research partnerships that integrate the research and development priorities of all participants; and

accelerate the exchange of research results within the network and the use of this knowledge within Canada by organisations that can harness it for Canadian economic and social development.

Contact:

Jean-Claude Gavrel / Networks of Centres of Excellence

www.nce.gc.ca

\subsubsection{Fl: Technology Programmes}

Technology programmes promote development in specific sectors of technology or industry, and the results of the research work are passed on the business systematically. „In technology programmes the focus is on technological research and development. These are extensive multi-annual co-operative projects comprising research projects by the universities, applied research, and corporate product development". The main benefits lay in the close cooperation between research institutes and industry, the widespread involvement of small and mediumsized companies, and the high level of international cooperation. Through the close cooperation with private companies in the preparation and steering of a programme, the business orientation of the programmes is guaranteed.

Contact:

$\underline{\text { www.tekes.fi }}$ 


\subsubsection{FR: PREDIT - Programme for research and innovation in land transport ${ }^{44}$}

PREDIT is a programme of research, experimentation and innovation in land transport, started and implemented by the ministries in charge of research, transport, environment and industry, the ADEME and the ANVAR. By stimulating cooperation between public and private sector, this programme aims at encouraging the creation of transportation systems that would be economically and socially more effective, safer, more energysaving, and finally more respectful of Man and environmentally-friendly.

PREDIT 1 (1990-1994) ) was mainly devoted to technological innovations in vehicles: TGV, automobile vehicles, light automatic underground trains. This first programme was granted 2.6 billion Francs (396.4 million Euros) in public funds.

PREDIT 2 (1996-2000) covered a noticeably wider field, considering the subjects concerned (involvement of Man and Society sciences, Life sciences...), but also the aims of the research (transport services organisation, goods, harbour interfaces...). This second programme made about 1400 research projects possible and received over 300 million Euros in public funds.

PREDIT 3 (2002-2006) will be marked by a specific effort put on goods transportation and energy and environment issues, greenhouse effect in particular, as well as a diversified research on safety. This third programme has been given about 300 million Euros in public funds.

PREDIT 3 is channelled by three general objectives:

To ensure the sustained mobility of people and goods,

To increase the safety of transportationsystems,

To reduce environmental impacts and contribute to the fight against the greenhouse effect.

Contact:

www.predit.prd.fr

${ }^{44}$ Programme de Recherche et d'Innovation dans les Transports terrestres 


\subsection{NL: TS - Technological Cooperation ${ }^{45}$}

The mission of TS is to strengthen R\&D by companies and to promote cooperation between businesses themselves or between companies and (semi-) public research institutes.

The TS programme is composed of a generic R\&D component and a complementary measure to support specific areas where international cooperation is deemed to be of strategic importance to the Netherlands. These include grants to support EUREKA projects, market research and cooperative projects to support engagement with emerging markets, cooperation within the maritime sector and cooperation in the area of ICT-breakthrough projects. In addition to its mainstream grants, TS sponsors measures such as networking, internationalisation and knowledge transfer.

\section{Contact:}

$\underline{\text { www.senter.nl }}$

\subsubsection{NO: TEFT - Technology Transfer from R\&D institutions to SMEs ${ }^{46}$}

TEFT is a nation-wide programme run by the Research Council of Norway (RCN). The programme is oriented towards small and medium-sized enterprises (SMEs) and research institutes. Concretely, it aims

to strengthen the ability of SMEs to initiate R\&D projects (business development),

to stimulate research institutes to orient themselves more strongly towards the needs of SMEs, to increase collaboration and thus transfer knowledge more effectively (infrastructure development).

\section{$\underline{\text { Contact }}$}

www.snd.no

\footnotetext{
${ }^{45}$ Technologische Samenwerking
}

${ }^{46}$ Teknologiformidling fra Forskningsinstitutter til små og mellomstore bedrifter 


\subsection{Literature}

Alexander (1995): How Organisations Act Together: Interorganisational Coordination in Theory and Practice (Amsterdam)

Argyris / Schon (1978): Organisational learning: A theory of action perspective, Reading MA.

Arnold / Boekholt / Keen (1995): Good Ideas in Programme Management for Research and Technical Development Programmes, Brighton

Arnold / Thuriaux / Lavery (1996): Putting Users in charge of R+D. International Experience of State Actions, Brighton

Barabasi (2002): Linked. The New Science of Networks, Cambridge (Mass.)

Boekholt, Arnold, et. al. (2002): The Governance of Research and Innovation. An international comparative study, Synthesis report Amsterdam

Boekholt, et. al. (1998): An International Comparative Study on Initatives to Build, Develop and Support "Kompetenzzentren", Brighton

Braun (1993): Who governs intermediary in reserch agencies Principla - agent relation policy making in: Journal of public policy, 15 (2) $135 \mathrm{ff}$

Bührer et. al. (1999): Kommunikation und Kooperation in interdisziplinären Zentren für klinische Forschung an Hochschulkliniken, Stuttgart

Bührer (2002): Network Analysis as an Evaluation Instrument: Methodes and Examples, EPUB, RTD Evaluation Toolbox, Spain (CD-Rom)

Bührer et. al. (2002): Die Kompetenzzentren der Nanotechnologie in der Frühphase der Bundesförderung. Ein Bericht der begleitenden Evaluation, Karlsruhe.

Burt (2003): Social Origins of Good Ideas, Chicago (University of Chicago, http://gsbwww.uchicago.edu/fac/ronald.burt/research/SOGI.pdf)

Caracostas / Muldur (1998): Society, The endless frontier. A European vision of research and innovation policies for the $21^{\text {st }}$ century. European Commission (Brussels)

Coase (1960/1988): The Firm, the Market, and the Law, The University of Chicago Press, (Chicago)

Caracostas 12003): Shared Governance through mutual Policy learning, in Changing Governance of Research and Technology Policy

Commission of the European Communities, COM (2001) 428 final (Brussels) 2001

De la Mothe, (2001): Knowledge, Politics and Governance; in: in: De la Mothe, J. (ed.), Science, Technology and Governance (London - New York), 3 ff

ETAN (1999): Options and Limits for Assessing the Socio-Economic Impact of European RTD Programmes, Report to the European Commission. 
European Commission (1999): Evaluating socio-economic programmes, MEANS collection Luxembourg.

\section{European Commission (2000): Towards a European Research Area}

Féron, Crowley (2002): Internationalization and the Governance of Research; in: Innovation The European Journal of Social Science Research, vol 15, Nr. 4 (Vienna)

Fukuyama, Wagner (2001): Governance Challenges of Technological Revolutions; in: De la Mothe, J. (ed.), Science, Technology and Governance (London - New York), 188 ff.

Georghiou / Roessner (2000): Evaluating technology programs: tools and methods. In: Research Policy 29, $657 \mathrm{ff}$

Hart, (2001): Governmental Organisation and Implications for Science and Technology Policy; in: De la Mothe, J. (ed.), Science, Technology and Governance (London - New York), 88 ff.

Hjorth (1998): The NUTEK Competence Centre Programme - An effort to build bridges between science and industry

ISI / KMFA (2004): Assessment "Zukunft der Kompetenzzentrenprogramme"; mimeo project report for Austrian ministries BMVIT and BMWA, Karlsruhe - Vienna

Kickert (1997): Managing Complex Networks, Strategies for the Public Sector, London

Kline, Rosenberg (1986): An overview of innovation. In: R. Landau; N. Rosenberg (Hg.): The Positive Sum Strategy: Harnessing Technology for Economic Growth. Washington D.C., S. 275305.

Kuhlmann (1998): Moderation of Policy-making? Science and Technology Policy Evaluation beyond Impact Measurement: the Case of Germany. In: Evaluation (Sage), Vol. 4, No. 2, April 1998, $130 \mathrm{ff}$

Kuhlmann (1999): Evaluation of Research Policy as a Moderation Process. In: Science Evaluation and It s Management, edited by V. Paces, L. Pivec, A.H. Teich. IOS Press, $8 \mathrm{ff}$

Kuhlmann (1999): Politisches System und Innovationssystem in "postnationalen" Arenen. In: Grimmer, K. /Kuhlmann, S. / Meyer-Krahmer, F. (eds.): Innovationspolitik in globalisierten Arenen, Leverkusen, $9 \mathrm{ff}$

Kuhlmann / Bührer (Ed.) (1999): Evaluation of Science and Technology in the New Europe. Proceedings of an International Conference. on 7 and 8 June, Berlin.

Kuhlmann, Edler (2003): Governance of Technology and Innovation Policies in Europe: Investigating Future Scenarios, in: Edler, J., Kuhlmann, S., Behrens, M. (ed.), Changing Governance of Research and Technology Policy. The European Research Area (Cheltenham Northampton) 2003, $3 \mathrm{ff}$.

Kuhlmann, (1998): Politikmoderation. Evaluationsverfahren in der Forschungs- und Technologiepolitik (Baden-Baden)

Lundvall (1992): National Systems of Innovation: Towards a Theory of Innovation and Interactive Learning, London. 
Lundvall, (2002) Innovation, Growth and Social Cohesion. The Danish Model (Cheltenham Northampton)

Mayntz, (1998): New Challenges to Governance Theory, Jean Monnet Chair Paper RSC No 98/50; http://www.iue.it/RSC/Mayntz.htm,

Meyer-Krahmer / Schmoch (1998): Science based technologies: university - industry interactions in four fields. In: Research Policy 27, $835 \mathrm{ff}$

Mintzberg (1973): The nature of managerial work, New York

Mittelstraß, (1994): Grundlagen und Anwendungen - Über das schwierige Verhältnis zwischen Forschung, Entwicklung und Politik. In: Chem.-Ing.Tech. 66, Nr. 3, 309-315.

Mumford / Robinson / Stradling (1987): Developing directors: the learning processes. Sheffield, The Manpower Commission

Narin (1997): The Increasing Linkage Between Technology and Public Science, Research Policy 1997, Vol 26, pp. 317-330.

Nelson (1993): National Innovation Systems, New York

North, (1992): Institutions, Institutional Change and Economic Performance (Cambridge).

OECD (1995): Impacts of National Technology Programmes

OECD (1997): Policy Evaluation in Innovation and Technology. Towards Best Practices;

OECD (1998): Technology, Productivity and Job Creation. Best Policy Practices

OECD (1998): University/Industry Research Partnerships: Typology and Issues [DSTI/STP/SUR(98)4]

OECD (1999): The knowledge based Economy. A Set of Facts and Figures

OECD (2001): Innovative Networks. Co-operation in National Innovation Systems, Paris

OECD (Hg.) (1999): Boosting Innovation: The Cluster Approach. Paris.

Ohler / Arnold et.al. (1998): Evaluation of the ITF Transport Technology Programme

PHARE (1995): Project Slovenia Expert Performance of firms with R\&D input, Ljubljana

Polt, (2001): The Role of Governments in Networking; in: Innovative Networks. Co-operation in National Innovation Systems. OECD (Paris)

Rammert (1997): Innovation im Netz. Neue Zeiten für technische Innovationen: heterogen verteilt und interaktiv vernetzt. In: Soziale Welt 48, S. 397-416.

Rose, (2001): Future Governance: Lessons from Comparative Public Policy. Ten steps in learning lessons from abroad. ESRC Future Governance Discussion Paper 1 (Glasgow)

Rose, (1993): Lesson-Drawing in Public Policy. A Guide to Learning Across Time and Space (Chatham, N.J.)

Saxenian, Annelee (1994): Regional Advantage. Culture and Competition in Silicon Valley and Route 128. Cambridge, Mass.: Harvard University Press. 
Schibany / Jörg / Polt (1999): Towards Realistic Expectations. The Science System as a Contributor to Industrial Innovation. Austrian TIP study

Schmoch, (1996): Die Rolle der akademischen Forschung in der Technikgenese, in: Soziale Welt 47, 2, 250-265.

Schmoch, (2003): Hochschulforschung und Industrieforschung. Perspektiven der Interaktion, Frankfurt/M. (Campus, Bd. 858).

Scott, (2001): Institutions and Organisations (Thousand Oaks - London - New Delhi), $2^{\text {nd }}$ ed.,.

Stampfer, (2003): European Research Area: New Roles for National and European RTDI Funding Programmes? In: Edler, J., Kuhlmann, S., Behrens, M. (ed.), Changing Governance of Research and Technology Policy. The European Research Area (Cheltenham - Northampton), $135 \mathrm{ff}$.

Stampfer, (2003a): Mehrebenenprogramme in Österreich - Konzeption, Rahmenbedingungen und Evaluierungsanforderungen anhand aktueller Beispiele aus der österreichischen Technologiepolitik; In: Bührer, S., Kuhlmann, S., Politische Steuerung von Innovationssystemen? (Stuttgart) S. $45-76$

Stampfer (2003b): European Research Area: New Roles for National and European Funding Programs?, in Edler, J., Kuhlmann., S., Behrens, M. (Eds.), Changing Governance of Research and Technology Policy. The European Research Area, Cheltenham - Northampton, 135 - 164

Stenberg (1997): Learning and policy development at STU/NUTEK - Competence Centres as an example. Paper presented at workshop of Advanced Science and Technology Policy Planning (ASTPP) Network, 26-28 May 1997 Bordeaux, France.

Technopolis / VDI-VDE / IKEI / Logotech (1999): Cross-Border Co-operation within National RTD Programmes 


\subsection{Comparability Sheets}

12.5.1 Comparability of MAPs in MAP-TN

12.5.2 Design of MAPs

12.5.3 Selection Procedures for MAPs

12.5.4 Proposal Evaluation of MAPs

12.5.5 $\quad$ Contract Negotiations in MAPs

12.5.6 Monitoring and Evaluation in MAPs

12.5.7 $\quad \underline{\text { StarMAPs }}$ 
12.3.1 Comparability of MAPs in MAP-TN (2002)

\begin{tabular}{|c|c|c|c|c|c|}
\hline & & & & & \\
\hline AT & TIG & \begin{tabular}{|l} 
Kplus \\
Competence \\
Centre \\
Programme
\end{tabular} & $\begin{array}{l}\text { COMPULSORY! long-term, Centres } \\
4+3 \text { years based on research plans, } \\
\text { EU-notified }\end{array}$ & $\begin{array}{l}\text { yes, PM-level: besides funding } \\
\text { extensive management help (learning } \\
\text { projects, workshops); Centre-level: } \\
\text { RTD projects, resource-development, } \\
\text { training courses, knowledge about } \\
\text { IPR, internationalisation-activities, } \\
\text { changing research culture }\end{array}$ & $\begin{array}{l}\text { yes, industry, science (univ., research } \\
\text { centres); intermediate organisations } \\
\text { play also important role in some } \\
\text { Centres ... Multi Firm Criterium }\end{array}$ \\
\hline SE & Vinnova & \begin{tabular}{|l|} 
Competence \\
Centre \\
Programme
\end{tabular} & COMPULSORY! core of programme & $\begin{array}{l}\text { yes, RTD projects, HR development, } \\
\text { leadership training (develop skills), } \\
\text { indirect: influence on universities to } \\
\text { organise big collaborations, in future } \\
\text { also hub for international } \\
\text { cooperations ... }\end{array}$ & $\begin{array}{l}\text { yes, university groups (no contract } \\
\text { research organisations) and industry, } \\
20 \% \text { hi tech SME; important is } \\
\text { personal involvement of industrial } \\
\text { RTD people; multi firm criterium }\end{array}$ \\
\hline EE & & \begin{tabular}{|l} 
Competence \\
Centre \\
Programme
\end{tabular} & & & \\
\hline DE & FhG-ISI & $\begin{array}{l}\text { Nanotechnology } \\
\text { Competence } \\
\text { Centre } \\
\text { Programme }\end{array}$ & $\begin{array}{l}\text { COMPULSORY? within network } \\
\text { shaped CC }\end{array}$ & $\begin{array}{l}\text { yes and no - and one is missing: no } \\
\text { project funding but only direct } 1 \text { ) } \\
\text { funding of network costs; network } \\
\text { agencies get funding, responsible for } \\
\text { networking; } 2 \text { ) cooperations ... 3) } \\
\text { many horizontal activities were } \\
\text { expected (+ there was pressure), but } \\
\text { not extra funded .... Project funds by } \\
\text { ministry for ALL actors }\end{array}$ & $\begin{array}{l}\text { yes; science, industry, transfer } \\
\text { agencies, "all actors with relevant } \\
\text { competence" }\end{array}$ \\
\hline ES & $\begin{array}{l}\text { Asociación } \\
\text { Unitec }\end{array}$ & \begin{tabular}{|l} 
PCTI- \\
Cooperacion
\end{tabular} & $\begin{array}{l}\text { COMPULSORY! in "RDTI } \\
\text { programme" ... Consortia science - } \\
\text { industry }\end{array}$ & & $\begin{array}{l}\text { within "RDTI programme" - planned } \\
\text { that only consortia (of what?) can } \\
\text { propose projects }\end{array}$ \\
\hline SL & $\begin{array}{l}\text { Univ. of } \\
\text { Maribor }\end{array}$ & \begin{tabular}{|l|} 
Industrial \\
Clusters \\
Development \\
Programme \\
(Toolmakers)
\end{tabular} & $\begin{array}{l}\text { SME as target group, cooperation } \\
\text { with PMIS lab from Univ. Maribor ... } \\
\text { Also in other clusters involvement of } \\
\text { university groups }\end{array}$ & $\begin{array}{l}\text { yes; University and enterprises } \\
\text { (leading enterprises and mostly SME) } \\
\text { in all } 3 \text { clusters: logistics, automotive, } \\
\text { toolmaker (tool and die; had a } \\
\text { forerunner in beginning of nineties); } \\
\text { Entrepreneurial Center as support }\end{array}$ & $\begin{array}{l}\mathrm{R}+\mathrm{D} \text {, marketing, internationalisation, } \\
\text { incubators ... "all business functions } \\
\text { including } \mathrm{R}+\mathrm{D} \text {... To the set up of } \\
\text { new companies"; }\end{array}$ \\
\hline IE & $\begin{array}{l}\text { NLR (on behalf } \\
\text { of the IEC) }\end{array}$ & Energy & $\begin{array}{l}\text { consortia include variable design (but } \\
\text { science - industry consortia is a } \\
\text { target), but bring in also local } \\
\text { governments and finance }\end{array}$ & $\begin{array}{l}\text { yes, studies, projects, international co- } \\
\text { operations: all on four topics } \\
\text { including House of Tommorrow, } \\
\text { Renewables and Supply (CHP) etc. } \\
\text { Also on improved skills (HR } \\
\text { developm.); infrastructure (support } \\
\text { networks) ... Policy networks }\end{array}$ & $\begin{array}{l}\text { yes; target groups are defined; } \\
\text { programme addresses industry, other } \\
\text { business (manufacturing and } \\
\text { services), research bodies, local } \\
\text { authorities, ... Feedback into policy } \\
\text { leads to other measures }\end{array}$ \\
\hline \begin{tabular}{|l|} 
BEI \\
Flanders
\end{tabular} & IIWT & \begin{tabular}{|l} 
VIS-CIN \\
(Cooperative \\
Innovation \\
Networks)
\end{tabular} & $\begin{array}{l}\text { in most of consortia also scientific } \\
\text { organisations and other non profit } \\
\text { organisations }\end{array}$ & $\begin{array}{l}\text { yes; RTD and Innovation funding: } 4 \\
\text { types of projects- 1) u. 2) thematic } \\
\text { Innovation stimulation: tech. advice } \\
\text { for firms with common needs (whole } \\
\text { Flanders but also sub-regional); } 3 \text { ) } \\
\text { Technological Service: Consultants } \\
\text { attached to Research Institutes - for } \\
\text { firms; 4) Collective Research Projects }\end{array}$ & $\begin{array}{l}\text { yes, at least } 20 \text { enterprises in one } \\
\text { network (no incorporation but strong } \\
\text { agreement) - with spokesman; or } \\
\text { existing organisations can be used; }\end{array}$ \\
\hline
\end{tabular}




\begin{tabular}{|c|c|c|c|c|c|}
\hline & & & & & \\
\hline Country & Organisation & MAP & 4. Public calls & $\begin{array}{l}\text { 5. External programme manager } \\
\text { (organisation) }\end{array}$ & $\begin{array}{l}\text { 6. Types of related activities } \\
\text { (accompanying measures) }\end{array}$ \\
\hline$\widehat{A T}$ & TIG & \begin{tabular}{|l} 
Kplus \\
Competence \\
Centre \\
Programme
\end{tabular} & yes, regular calls, 2 stage-evaluation & $\begin{array}{l}\text { yes, TIG as overall programme } \\
\text { manager }\end{array}$ & $\begin{array}{l}\text { yes; additional activities of Centres } \\
\text { ("Non K plus") }\end{array}$ \\
\hline SE & Vinnova & $\begin{array}{l}\text { Competence } \\
\text { Centre } \\
\text { Programme }\end{array}$ & yes, one big call (two stage) & $\begin{array}{l}\text { yes, VINNOVA responsible for whole } \\
\text { programme; programme created } \\
\text { within NUTEK; CC programme } \\
\text { survived agency change }\end{array}$ & yes, new management approaches \\
\hline $\mathrm{EE}$ & & \begin{tabular}{|l} 
Competence \\
Centre \\
Programme
\end{tabular} & & & \\
\hline$\overline{D E}$ & FhG-ISI & $\begin{array}{l}\text { Nanotechnology } \\
\text { Competence } \\
\text { Centre } \\
\text { Programme }\end{array}$ & $\begin{array}{l}\text { yes, but no competition ; review } \\
\text { panels }\end{array}$ & $\begin{array}{l}\text { yes, "Projektträger": two for } \\
\text { programme }\end{array}$ & \begin{tabular}{|l|} 
FhG-ISI came in as kind of mediator \\
and accompanying evaluator ... "but \\
too late", "already highly disturbed \\
relations between Centers and \\
ministry"; funding of research projects
\end{tabular} \\
\hline ES & $\begin{array}{l}\text { Asociación } \\
\text { Unitec }\end{array}$ & $\begin{array}{l}\text { PCTI- } \\
\text { Cooperacion }\end{array}$ & yes, planned in "RTDI programme" & $\begin{array}{l}\text { Basque ministry has unit for } \\
\text { programme }\end{array}$ & \\
\hline SL & $\begin{array}{l}\text { Univ. of } \\
\text { Maribor }\end{array}$ & \begin{tabular}{|l|} 
Industrial \\
Clusters \\
Development \\
Programme \\
(Toolmakers)
\end{tabular} & yes, open calls for interesting clusters & $\begin{array}{l}\text { no; MoE (economics ministry for } \\
\text { econ) }\end{array}$ & $\begin{array}{l}\text { international co-operation with e.g. } \\
\text { Austrian Clusters; interplay within } \\
\text { regional and innovation policy }\end{array}$ \\
\hline IE & $\begin{array}{l}\text { NLR (on behalf } \\
\text { of the IEC) }\end{array}$ & Energy & $\begin{array}{l}\text { yes, open calls for majority of project } \\
\text { types.... } 2 \text { stage calls }\end{array}$ & $\begin{array}{l}\text { yes, IEC (SEAI) - also sets goals like } \\
\text { reduction goals, number of } \\
\text { demonstrations }\end{array}$ & Energy policy measures .... \\
\hline \begin{tabular}{|l|} 
BE/ \\
Flanders
\end{tabular} & IWT & \begin{tabular}{|l} 
VIS-CIN \\
(Cooperative \\
Innovation \\
Networks)
\end{tabular} & $\begin{array}{l}\text { yes, open call, committees with } \\
\text { external experts }\end{array}$ & $\begin{array}{l}\text { yes; IWT in charge of programme; } \\
\text { framework decided by government }\end{array}$ & $\begin{array}{l}\text { Programme itself is about a bunch of } \\
\text { "accompanying" and stimulating } \\
\text { measures; new networks shall come } \\
\text { into life; how much help shall they get } \\
\text { from funding organisation }\end{array}$ \\
\hline
\end{tabular}




\begin{tabular}{|c|c|c|c|c|}
\hline Country & Organisation & MAP & $\begin{array}{l}\text { 7. Types of evaluations: ex ante, } \\
\text { monitoring and ex post }\end{array}$ & $\begin{array}{l}\text { 8. Types of specific learning } \\
\text { elements and feedback loops }\end{array}$ \\
\hline AT & TIG & \begin{tabular}{|l} 
Kplus \\
Competence \\
Centre \\
Programme
\end{tabular} & $\begin{array}{l}\text { yes (all), } 2 \text { stage-evaluation, TIG } \\
\text { does financial monitoring; scientific } \\
\text { evaluation; programme-ev. not } \\
\text { carried out by TIG; centre-ev. was } \\
\text { clear before progr. started -... Other } \\
\text { monitoring within Centres (Boards) }\end{array}$ & $\begin{array}{l}\text { yes: e.g. SOL; yes workshops with } \\
\text { Centres and other management / } \\
\text { learning instruments, internat. Best } \\
\text { practice }\end{array}$ \\
\hline $\mathrm{SE}$ & Vinnova & \begin{tabular}{|l} 
Competence \\
Centre \\
Programme
\end{tabular} & $\begin{array}{l}\text { yes ... Big ex ante evaluation, regular } \\
\text { (year } 2-5-7-10 \text { ) evaluation .... } \\
\text { Monitoring on Centre level is Centre } \\
\text { responsibility (Boards) }\end{array}$ & $\begin{array}{l}\text { learning loops, step by step } \\
\text { (evaluations), organisational learning } \\
\text { in programme; strong role for } \\
\text { VINNOVA; foreign "standing" } \\
\text { evaluators; internat. Best practice }\end{array}$ \\
\hline $\mathrm{HU}$ & $\mathrm{OM} \mathrm{K}+\mathrm{F}$ & \begin{tabular}{|l|} 
KKK \\
Competence \\
Centre \\
Programme \\
\end{tabular} & yes, long ex ante procedure ... & internat. Best practice \\
\hline EE & & \begin{tabular}{|l|} 
Competence \\
Centre \\
Programme
\end{tabular} & & \\
\hline$\overline{D E}$ & FhG-ISI & \begin{tabular}{|l|} 
Nanotechnology \\
Competence \\
Centre \\
Programme
\end{tabular} & $\begin{array}{l}\text { yes; ex ante and .... mid term } \\
\text { evaluations; and monitoring / } \\
\text { evaluations .... See related activities }\end{array}$ & $\begin{array}{l}\text { role of FHG-ISI as accompanying } \\
\text { evaluators ... }\end{array}$ \\
\hline UK & Technopolis & \begin{tabular}{|l|} 
Partners in \\
Innovation (P II)
\end{tabular} & $\begin{array}{l}\text { ex ante evaluation rather quick; } \\
\text { monitoring with help from sector } \\
\text { experts; some audits; financial ex } \\
\text { post evaluation by project officers; } \\
\text { programme only partly reviewed }\end{array}$ & $\begin{array}{l}\text { increase transfer of knowledge ..... } \\
\text { Link to regulation }\end{array}$ \\
\hline ES & $\begin{array}{l}\text { Asociación } \\
\text { Unitec }\end{array}$ & \begin{tabular}{|l} 
PCTI- \\
Cooperacion
\end{tabular} & ex ante & new programme \\
\hline $\mathbf{S L}$ & \begin{tabular}{|l|} 
Univ. of \\
Maribor
\end{tabular} & $\begin{array}{l}\text { Industrial } \\
\text { Clusters } \\
\text { Development } \\
\text { Programme } \\
\text { (Toolmakers) }\end{array}$ & $\begin{array}{l}\text { reporting: yes, done by ministry, } \\
\text { formalised; project evaluation: yes, } \\
\text { done by ministry }\end{array}$ & $\begin{array}{l}\text { see: cross border co-operations with } \\
\text { other Clusters }\end{array}$ \\
\hline IE & $\begin{array}{l}\text { NLR (on behalf } \\
\text { of the IEC) }\end{array}$ & Energy & yes & no formal mechanism \\
\hline \begin{tabular}{|l|}
$\mathrm{BE} /$ \\
Flanders
\end{tabular} & IWT & $\begin{array}{l}\text { VIS-CIN } \\
\text { (Cooperative } \\
\text { Innovation } \\
\text { Networks) }\end{array}$ & $\begin{array}{l}\text { yes; number of indicators to monitor } \\
\text { and evaluate programme }\end{array}$ & \\
\hline
\end{tabular}


'12.3.2. Design of MAPs (March 2002)

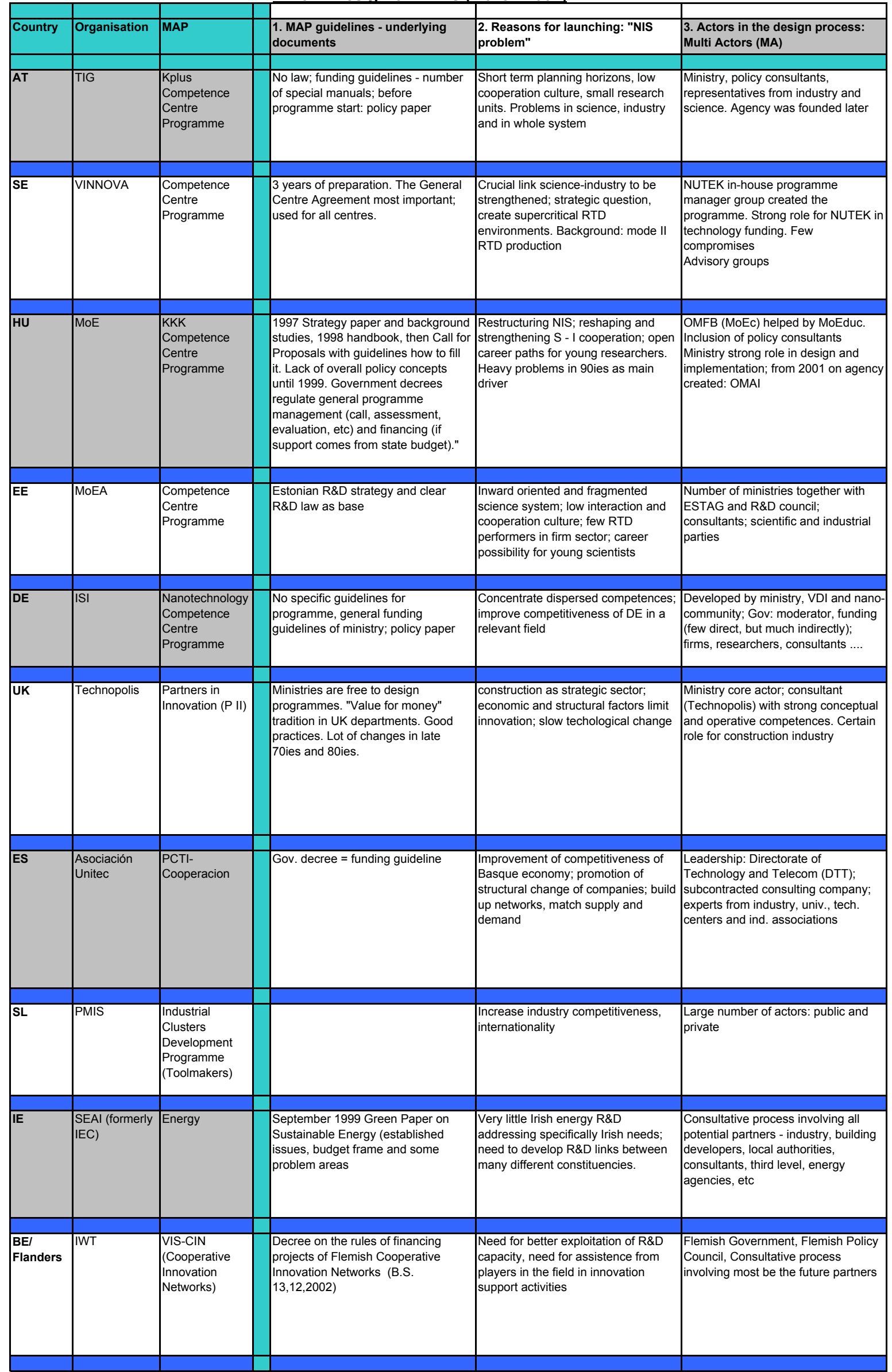




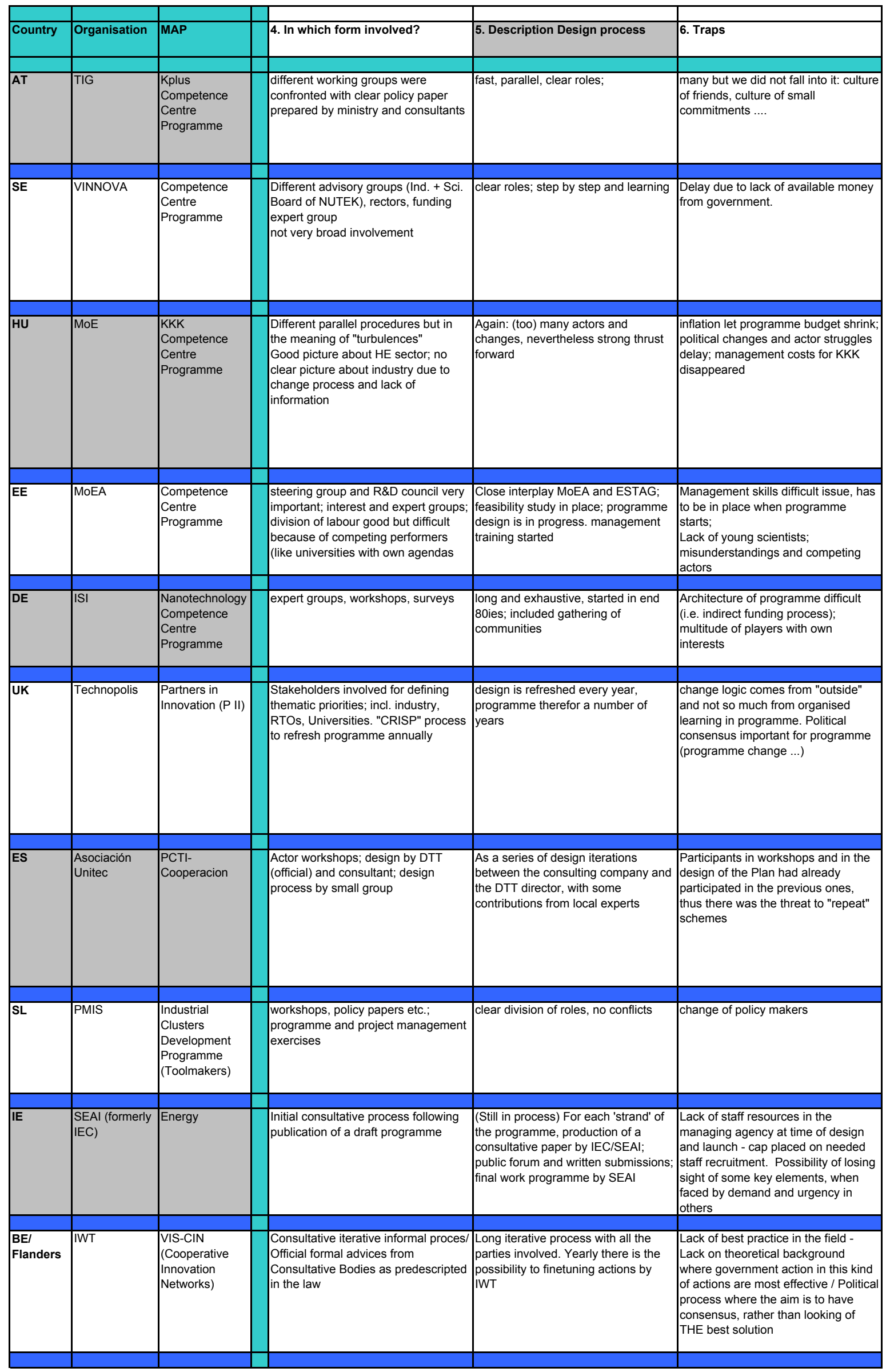




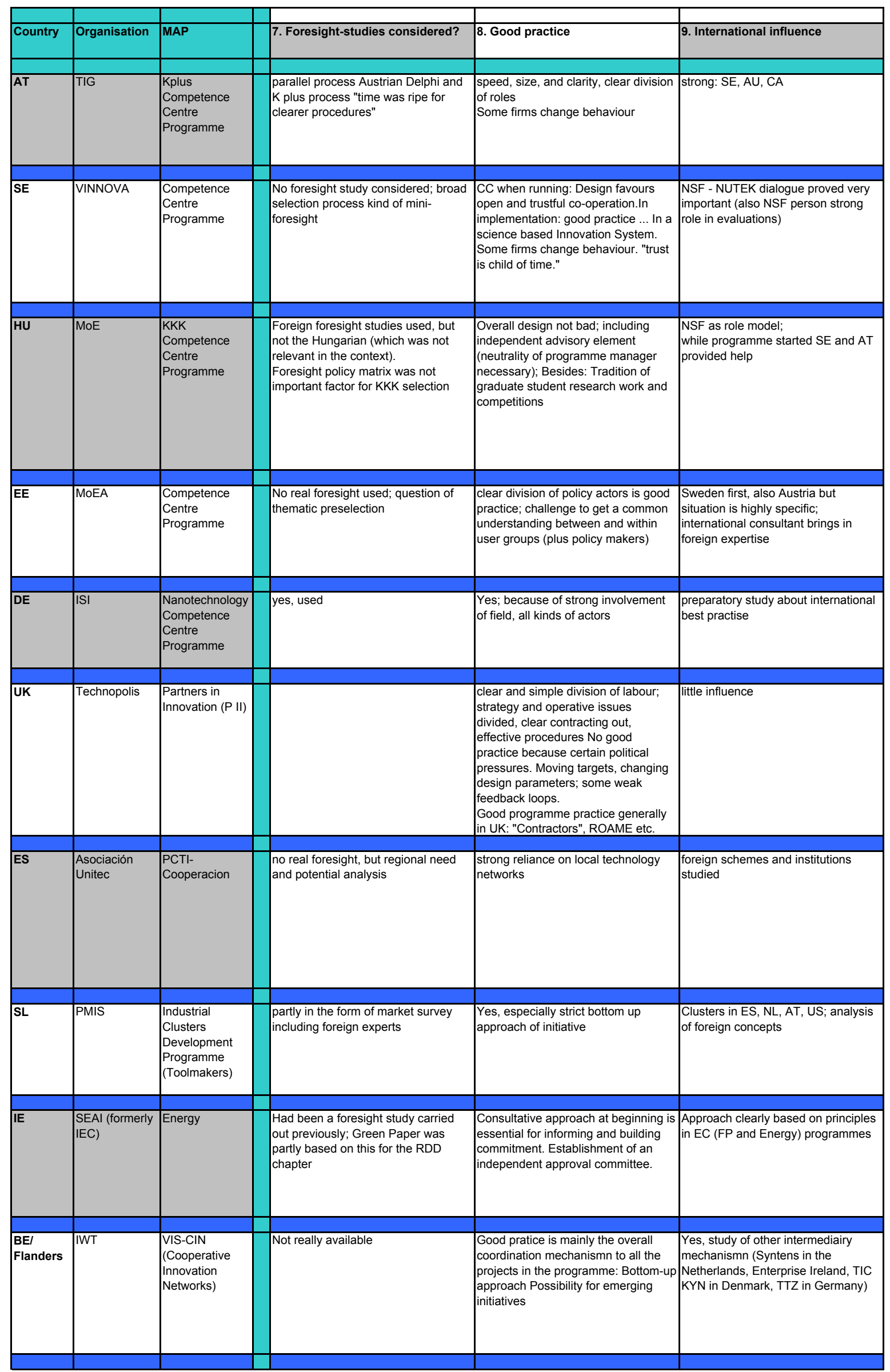




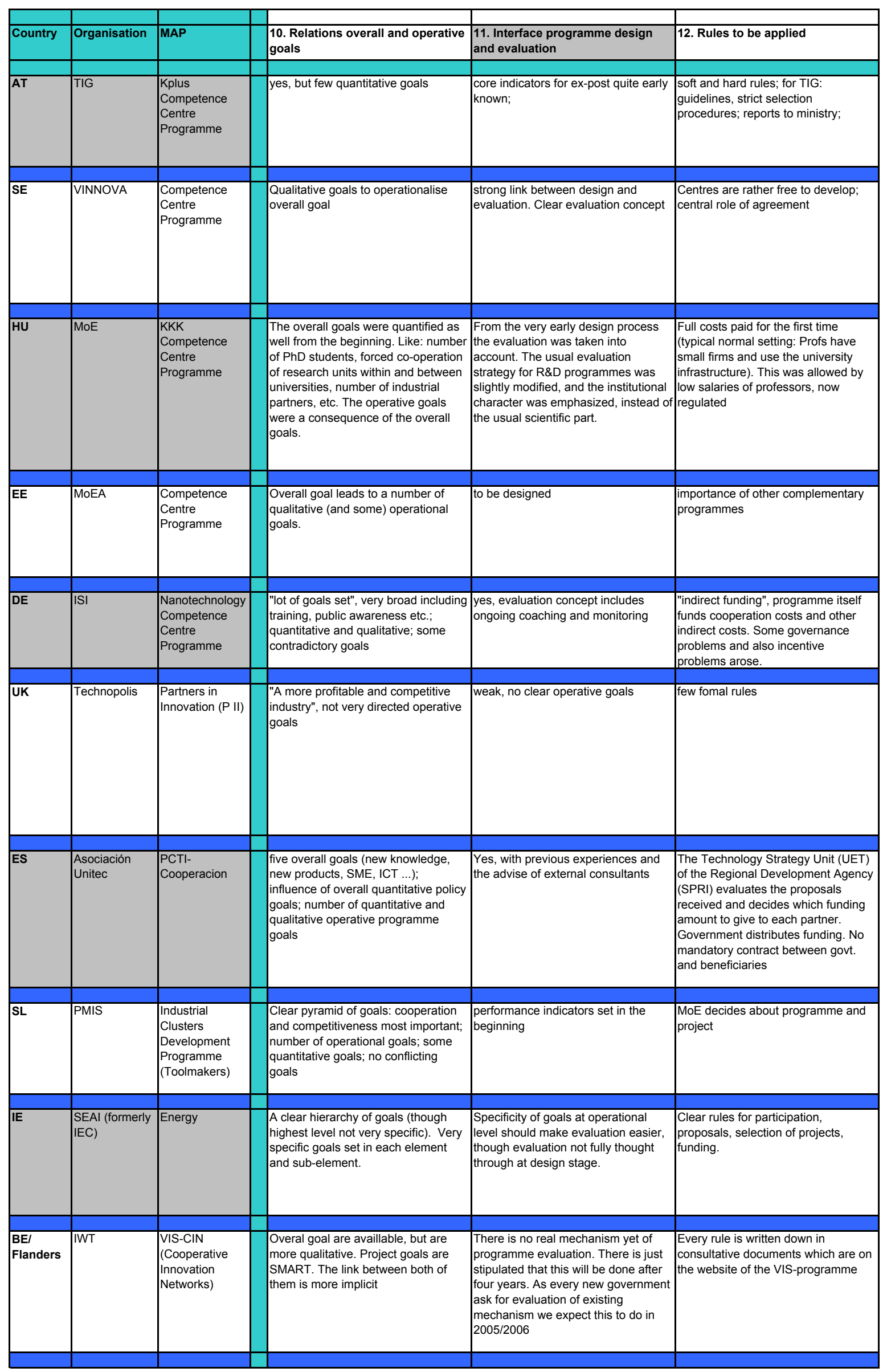




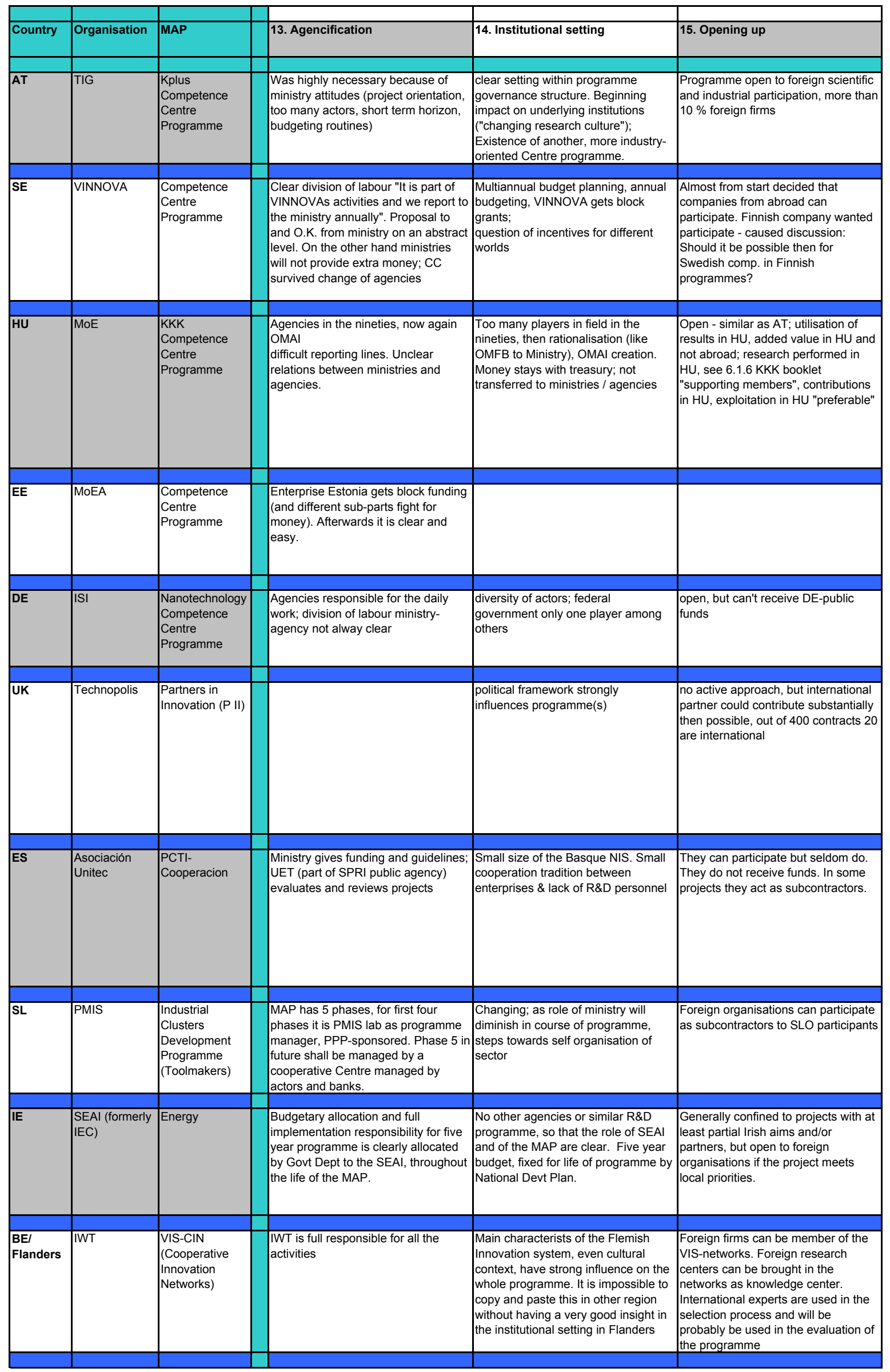


12.3.3 Selection Procedures for MAPs (June 2002)

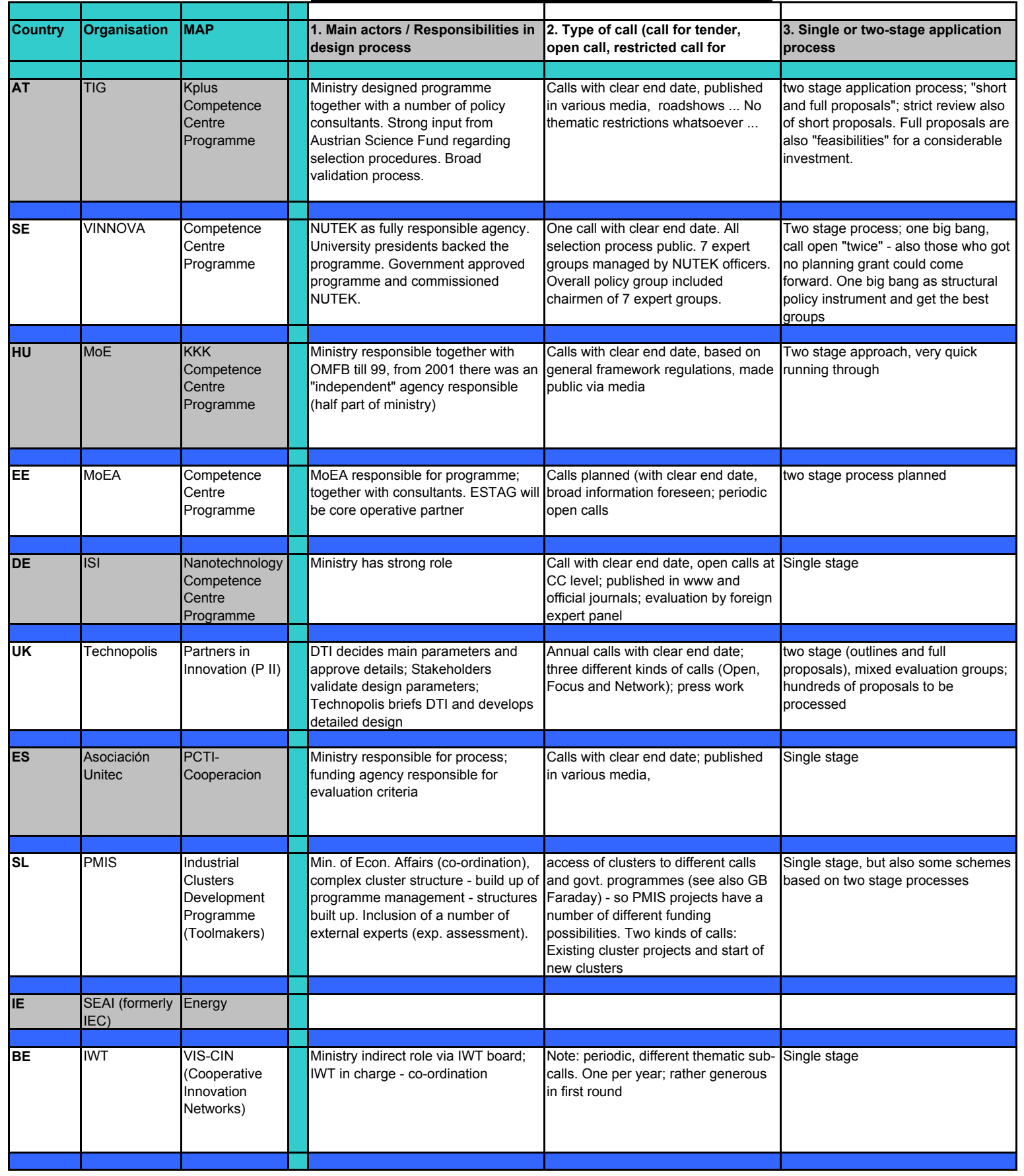




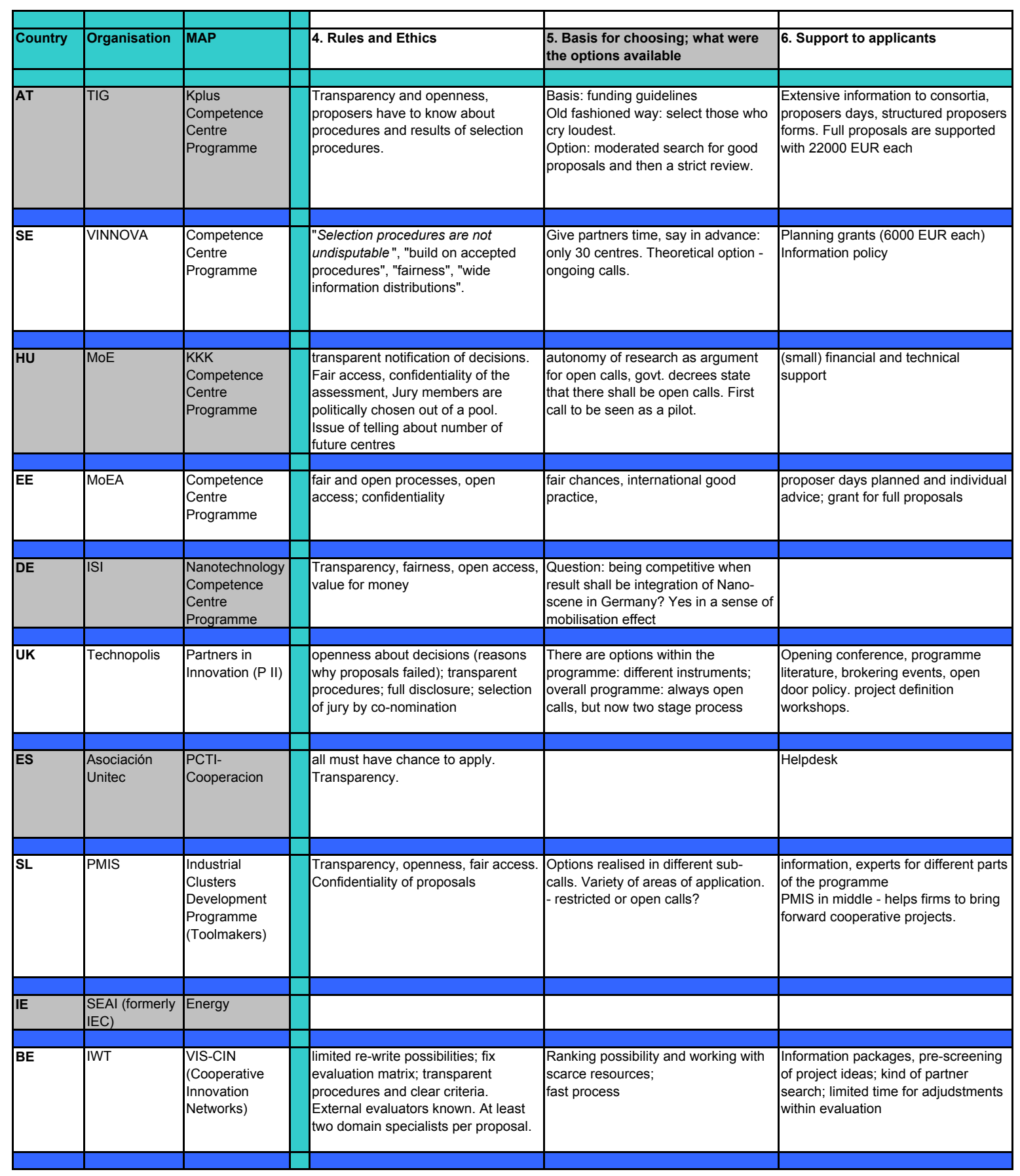




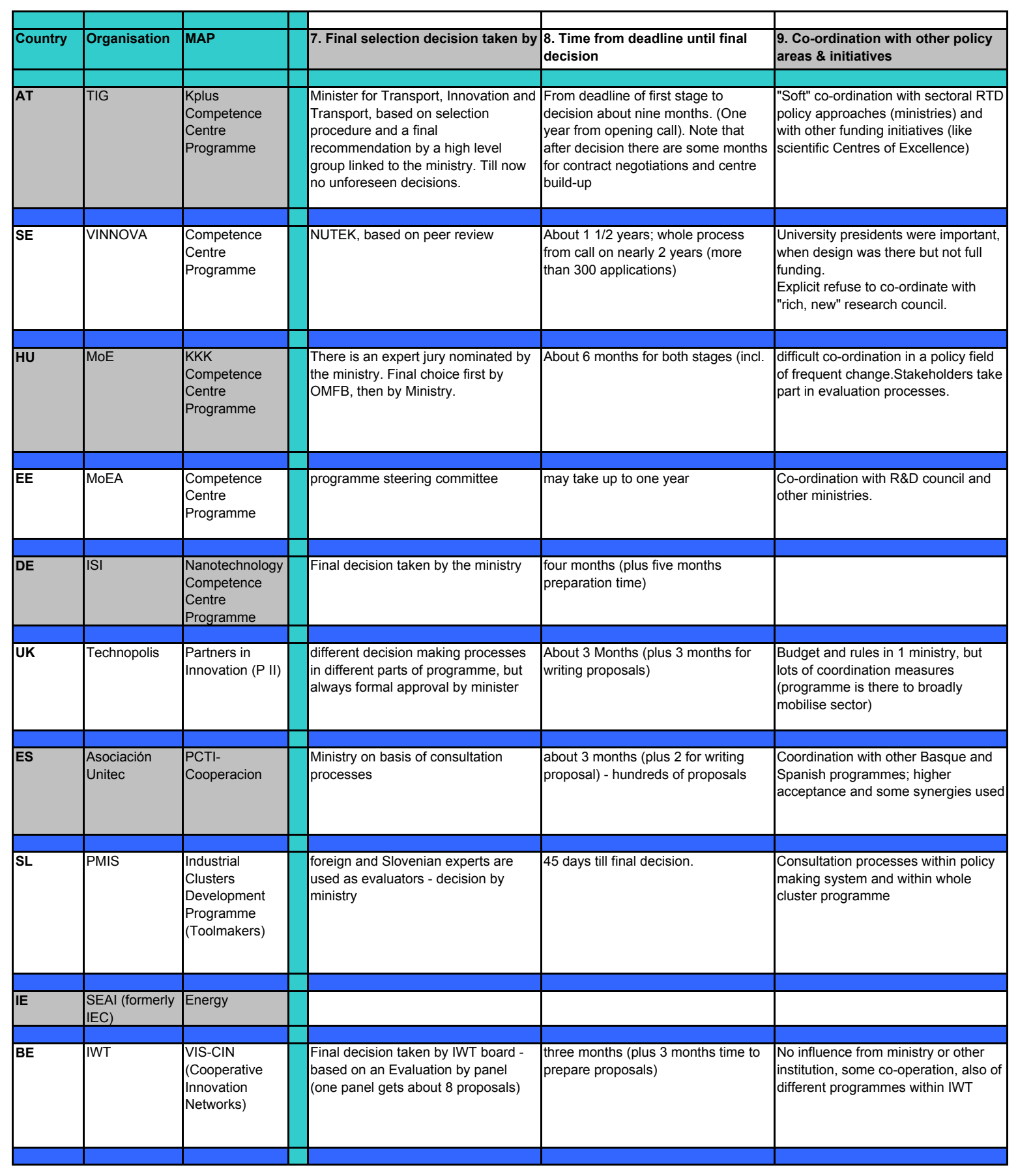




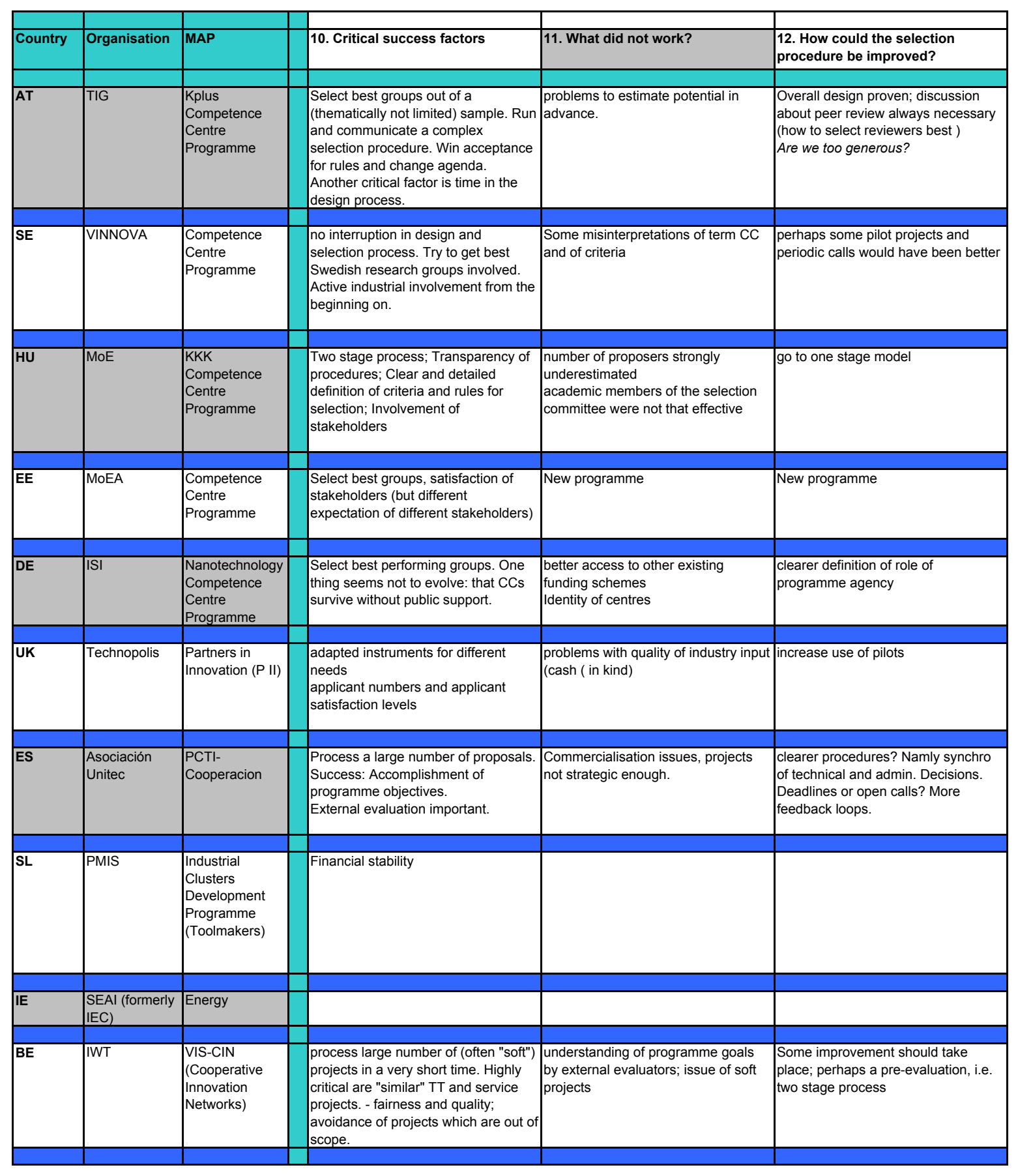




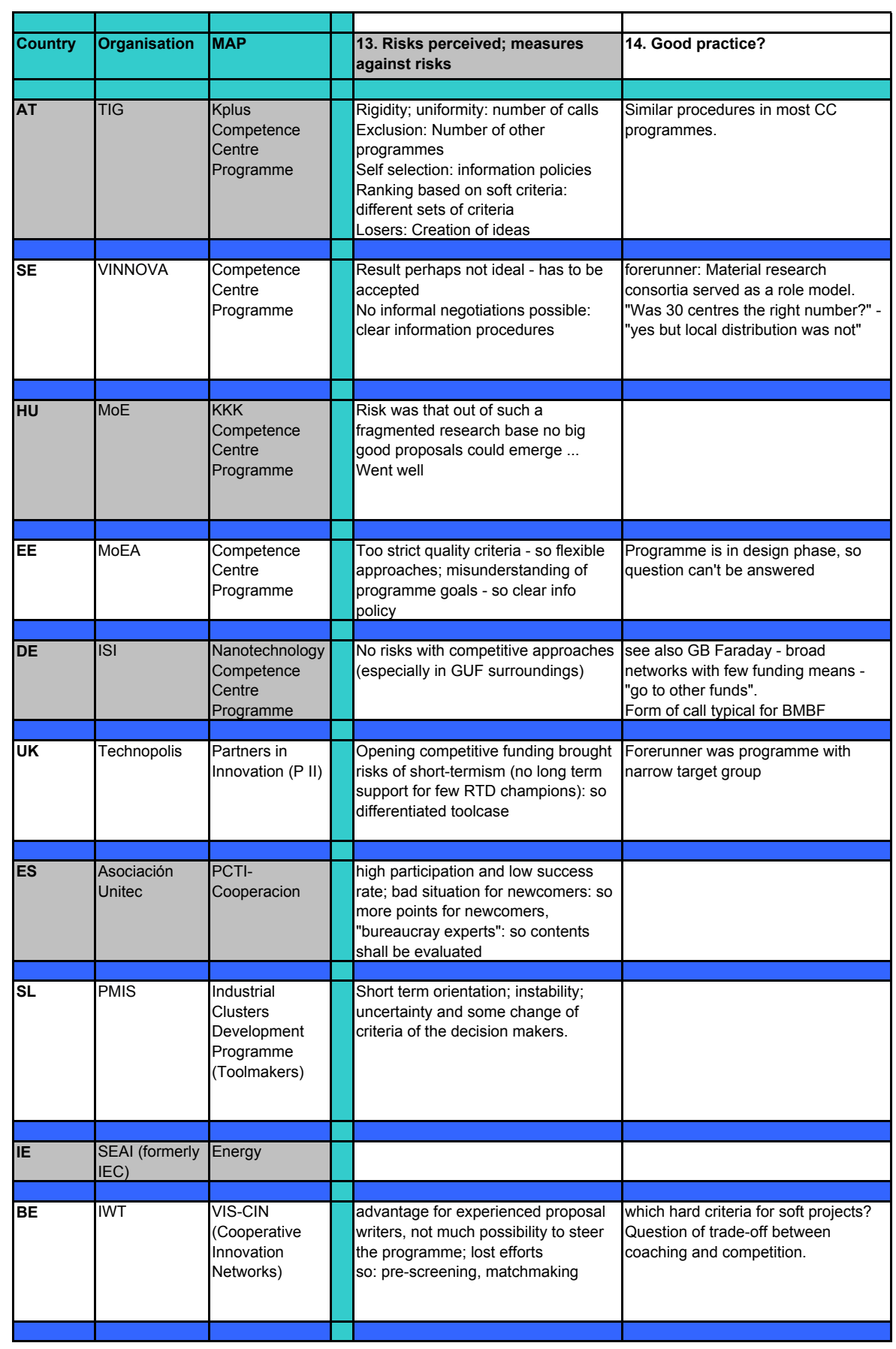




\begin{tabular}{|c|c|c|c|c|c|}
\hline & & & & & \\
\hline Country & Organisation & MAP & 1. Support strategy & 2. Scope of support & 3. Communication channels \\
\hline AT & TIG & \begin{tabular}{|l} 
Kplus \\
Competence \\
Centre \\
Programme
\end{tabular} & \begin{tabular}{|l|} 
internet, newspaper when call is \\
opened; never matchmaker, advice \\
on best practices; never advice on \\
technical details; financial support for \\
second stage
\end{tabular} & & $\begin{array}{l}\text { guidelines, homepage, offer to } \\
\text { present the programme at different } \\
\text { organisations, bilateral meetings; } \\
\text { proposer's days }\end{array}$ \\
\hline SE & VINNOVA & \begin{tabular}{|l|} 
Competence \\
Centre \\
Programme
\end{tabular} & $\begin{array}{l}\text { Only one selection round so far - } \\
\text { model for selection procedure was } \\
\text { taken from other programme } \\
\text { (materials); aim: encourage best } \\
\text { proposals and secure neutrality of } \\
\text { NUTEK }\end{array}$ & planning grant (EUR 6000.-) & \begin{tabular}{|l|} 
programme documentation and \\
written guidelines, NUTEK managers \\
available on request (15 managers)
\end{tabular} \\
\hline $\mathrm{HU}$ & $\begin{array}{l}\text { Ministry of } \\
\text { Education }\end{array}$ & \begin{tabular}{|l} 
KKK \\
Competence \\
Centre \\
Programme
\end{tabular} & $\begin{array}{l}\text { Consortia - higher funding volume + } \\
\text { educational programme influence } \\
\text { strategy }\end{array}$ & $\begin{array}{l}\text { Two-day seminar with researchers } \\
\text { from university, research institutes } \\
\text { and industry; KKK Committe: } \\
\text { brokering after the first round plus } \\
\text { comment on technical part; planning } \\
\text { grant for } 2 \text { nd round }(4000 €)\end{array}$ & \begin{tabular}{|l|} 
Newspapers, printed form, website \\
(=standard) plus direct mailing to \\
universities and research groups and \\
industry \\
long discussion process before \\
programme actually started
\end{tabular} \\
\hline $\mathrm{EE}$ & $\begin{array}{l}\text { Ministry of } \\
\text { Economic } \\
\text { Affairs }\end{array}$ & \begin{tabular}{|l|} 
Competence \\
Centre \\
Programme
\end{tabular} & \begin{tabular}{|l|} 
Information days, every-day \\
consultation with Programme \\
manager in EAS, general training, \\
specific training focusing on the \\
specificity of the competence centre \\
model, support grants for preparing of \\
the full proposals
\end{tabular} & $\begin{array}{l}\text { Revision of applications during the } \\
\text { full proposal phase. The process is } \\
\text { important not the static verification of } \\
\text { the applications at the end of the } \\
\text { application procedure. }\end{array}$ & $\begin{array}{l}\text { Newspapers, magazines, www; } \\
\text { information days, seminars; applicant } \\
\text { formal briefing; programme } \\
\text { documentation and case material }\end{array}$ \\
\hline $\mathrm{DE}$ & $\mid \mathrm{SI}$ & \begin{tabular}{|l|} 
Nanotechnology \\
Competence \\
Centre \\
Programme
\end{tabular} & $\begin{array}{l}\text { (informal) information delivered by } \\
\text { the ministry and agencies on "real } \\
\text { issues", assistance (if needed) in } \\
\text { finding relevant partners }\end{array}$ & informal information & $\begin{array}{l}\text { guidelines, homepage, personal } \\
\text { advice }\end{array}$ \\
\hline UK & Technopolis & \begin{tabular}{|l|} 
Partners in \\
Innovation (P II)
\end{tabular} & & 15.000 Pounds for large proposals; & \begin{tabular}{|l|} 
Booklets (wide dissemination: \\
participants, local communities, ... \\
website (with gov support databases); \\
feedback at the end of the procedure
\end{tabular} \\
\hline ES & $\begin{array}{l}\text { Asociacion } \\
\text { Unitec }\end{array}$ & $\begin{array}{l}\text { PCTI- } \\
\text { Cooperacion }\end{array}$ & $\begin{array}{l}\text { decree + recommendations - UET } \\
\text { officers (always prior to proposal } \\
\text { submission) }\end{array}$ & $\begin{array}{l}\text { Commenting early drafts. Industry } \\
\text { associations (clusters) organise } \\
\text { brokering activities where UET } \\
\text { officers take part. Participants are } \\
\text { supposed to be able to partner with } \\
\text { their own contacts, and to elaborate } \\
\text { excellent proposals. }\end{array}$ & $\begin{array}{l}\text { briefings for industry associations, } \\
\text { public conference and programme } \\
\text { documentation }\end{array}$ \\
\hline $\mathbf{S L}$ & PMSI-lab & $\begin{array}{l}\text { Industrial } \\
\text { Clusters } \\
\text { Development } \\
\text { Programme } \\
\text { (Toolmakers) }\end{array}$ & $\begin{array}{l}\text { Info on objectives and scope (funding } \\
\text { quota) from } \\
\text { programmemanagmenent, rules of } \\
\text { business }\end{array}$ & formal and informal & $\begin{array}{l}\text { Official printed material (aim, } \\
\text { purpose, evaluations, ...), }\end{array}$ \\
\hline$\overline{I E}$ & SEI & Energy & & $\begin{array}{l}15.000 \text { euros potentially available for } \\
\text { feasibility studies. }\end{array}$ & $\begin{array}{l}\text { Printed info packs; website and } \\
\text { downloadable forms etc }\end{array}$ \\
\hline $\mathrm{BE}$ & IIWT & \begin{tabular}{|l|} 
VIS-CIN \\
(Cooperative \\
Innovation \\
Networks)
\end{tabular} & $\begin{array}{l}\text { decree + documents on how to } \\
\text { submit a proposal - information and } \\
\text { communication sessions to possible } \\
\text { participants - possibilty to have of } \\
\text { prescreening of a proposal }\end{array}$ & Formal and informal & $\begin{array}{l}\text { Webiste, newsletter, information } \\
\text { sessions, individual appointments }\end{array}$ \\
\hline
\end{tabular}




\begin{tabular}{|c|c|c|c|c|c|}
\hline & & & & & \\
\hline Country & Organisation & MAP & 4. Format of outline proposal & 5. Format of full proposal & 6. Monitoring \& ex-post evaluation \\
\hline AT & TIG & \begin{tabular}{|l|} 
Kplus \\
Competence \\
Centre \\
Programme
\end{tabular} & 20 to 40 pages for "feasibility check" & $\begin{array}{l}\text { Projects for the first two years have to } \\
\text { be defined in detail }\end{array}$ & $\begin{array}{l}\text { for mid-term ev. Evaluators can have } \\
\text { also original proposals }\end{array}$ \\
\hline SE & VINNOVA & \begin{tabular}{|l|} 
Competence \\
Centre \\
Programme
\end{tabular} & $\begin{array}{l}\text { max. } 12 \text { pages - to save researchers } \\
\text { time ; very strong link with evaluation } \\
\text { criteria }\end{array}$ & $\begin{array}{l}\text { max. } 20 \text { pages (!), financial proposals } \\
\text { included but not evaluated }\end{array}$ & $\begin{array}{l}\text { NO. New and visionary concept - } \\
\text { outcome unclear, learning curve for } \\
\text { programme management }\end{array}$ \\
\hline $\mathrm{HU}$ & $\begin{array}{l}\text { Ministry of } \\
\text { Education }\end{array}$ & \begin{tabular}{|l} 
KKK \\
Competence \\
Centre \\
Programme
\end{tabular} & $\begin{array}{l}\text { Choice of proposals that seemed } \\
\text { viable form the cooperation side (not } \\
\text { only scientific quality); length: } 15-20 \\
\text { pages }\end{array}$ & $\begin{array}{l}\text { structure same outline proposal; } \\
\text { length: } 30 \text { pages - } 200 \text { pages incl. } \\
\text { Appendices; strong link to eval } \\
\text { criteria } \\
\text { "Strategy plus financial plan = } \\
\text { Business plan" (demanded by } \\
\text { industry) }\end{array}$ & $\begin{array}{l}\text { Contract negotiations: strategy and } \\
\text { financial plan is app. To contract - } \\
\text { strictly checked by monitoring system }\end{array}$ \\
\hline EE & $\begin{array}{l}\text { Ministry of } \\
\text { Economic } \\
\text { Affairs }\end{array}$ & \begin{tabular}{|l|} 
Competence \\
Centre \\
Programme
\end{tabular} & $\begin{array}{l}\text { The short and full proposals are } \\
\text { presented to EAS according to the } \\
\text { respective application forms (in paper } \\
\text { format, in electronic form). } \\
\text { Applications forms are very much in } \\
\text { compliance with the evaluation } \\
\text { criteria presented in the call. }\end{array}$ & $\begin{array}{l}\text { information on the research area and } \\
\text { the projects as per the conditions set } \\
\text { out under the operating principles of } \\
\text { the competence centres, general } \\
\text { conditions for the participants of the } \\
\text { competence centres, evaluation } \\
\text { criteria of competence centre } \\
\text { application and financing criteria of } \\
\text { the competence centres }\end{array}$ & $\begin{array}{l}\text { The applications are the } \\
\text { fundamentals of the establishment of } \\
\text { the competence centres. Based on } \\
\text { the semi-annual and annual reports } \\
\text { the efficiency of the competence } \\
\text { centres based on the first ideas in the } \\
\text { application incl. business plan are } \\
\text { evaluated. }\end{array}$ \\
\hline $\mathrm{DE}$ & $|S|$ & \begin{tabular}{|l|} 
Nanotechnology \\
Competence \\
Centre \\
Programme
\end{tabular} & single step procedure & \begin{tabular}{|l|} 
proposals differ substantially \\
reaching from 20 up to about 50 \\
pages. Core elements are objectives, \\
previous experiences, work plan, \\
success prospective (scientific and \\
technological), members of the CCN, \\
reasons for the applied means.
\end{tabular} & $\begin{array}{l}\text { proposals to refer to evaluation } \\
\text { criteria, for example mentioning the } \\
\text { intended activities in the field of } \\
\text { public relations }\end{array}$ \\
\hline UK & Technopolis & \begin{tabular}{|l|} 
Partners in \\
Innovation (P II)
\end{tabular} & $\begin{array}{l}4 \text { page outline, } 16 \text { page full proposal; } \\
\text { indicative letters of support; for full } \\
\text { proposal incl. Amount of money }\end{array}$ & & \\
\hline ES & $\begin{array}{l}\text { Asociacion } \\
\text { Unitec }\end{array}$ & $\begin{array}{l}\text { PCTI- } \\
\text { Cooperacion }\end{array}$ & There is no outline proposal & $\begin{array}{l}\text { Approx } 30 \text { pages. Technical and } \\
\text { financial aspects }\end{array}$ & $\begin{array}{l}\text { Workplan in proposal is link to } \\
\text { monitoring }\end{array}$ \\
\hline $\mathbf{S L}$ & PMSI-lab & $\begin{array}{l}\text { Industrial } \\
\text { Clusters } \\
\text { Development } \\
\text { Programme } \\
\text { (Toolmakers) }\end{array}$ & $\begin{array}{l}\text { chose among competing ideas, link } \\
\text { with programme scope, applicants } \\
\text { references }\end{array}$ & $\begin{array}{l}50-100 \text { pages, (min. } 20 \text { companies); } \\
\text { need to show depening of existing } \\
\text { cooperations; monitoring indicators }\end{array}$ & \begin{tabular}{|l} 
business goals and connected \\
indicators, project milestone plan
\end{tabular} \\
\hline IE & SEI & Energy & & $\begin{array}{l}\text { 9-page form, with detailed suggested } \\
\text { layout for proposals }\end{array}$ & $\begin{array}{l}\text { work programme with specified } \\
\text { milestones }\end{array}$ \\
\hline $\mathrm{BE}$ & IWT & $\begin{array}{l}\text { VIS-CIN } \\
\text { (Cooperative } \\
\text { Innovation } \\
\text { Networks) }\end{array}$ & single-step procedure & $\begin{array}{l}\text { set out in a document available on } \\
\text { the website }\end{array}$ & $\begin{array}{l}\text { project proposal has to describe the } \\
\text { monitoring and evaluation procedure } \\
\text { and indicators }\end{array}$ \\
\hline
\end{tabular}




\begin{tabular}{|c|c|c|c|c|c|}
\hline & & & & & \\
\hline Country & Organisation & MAP & $\begin{array}{l}\text { 7. Co-ordination body of evaluation } \\
\text { process }\end{array}$ & $\begin{array}{l}\text { 8. Procedures of evaluation } \\
\text { process }\end{array}$ & $\begin{array}{l}\text { 9. Final decisions for evaluation } \\
\text { process }\end{array}$ \\
\hline$\overline{A T}$ & TIG & \begin{tabular}{|l} 
Kplus \\
Competence \\
Centre \\
Programme
\end{tabular} & TIG acts as secretariat & $\begin{array}{l}\text { eligibility check by TIG, strictly } 3 \\
\text { internat. Peers; (for short proposal); } \\
6 \text { int. Scientific reviewers .... And } \\
\text { visiting committees - on day } \\
\text { discussion and feedback loop }\end{array}$ & $\begin{array}{l}\text { Minister based on recommendations } \\
\text { of the jury }\end{array}$ \\
\hline SE & VINNOVA & \begin{tabular}{|l|} 
Competence \\
Centre \\
Programme
\end{tabular} & \begin{tabular}{|l|} 
NUTEK, arrangement with advisory \\
groups (7 expert groups, central \\
policy group, meetings with university \\
and industrial groups)
\end{tabular} & & $\begin{array}{l}\text { NUTEK based on recommendations, } \\
\text { report to government }\end{array}$ \\
\hline $\mathrm{HU}$ & $\begin{array}{l}\text { Ministry of } \\
\text { Education }\end{array}$ & $\begin{array}{l}\text { KKK } \\
\text { Competence } \\
\text { Centre } \\
\text { Programme }\end{array}$ & $\begin{array}{l}\text { OMFB / Ministry of Education; in fact: } \\
\text { agency }\end{array}$ & $\begin{array}{l}\text { Compliance test (Clearing with other } \\
\text { funding organisations); KKK Comittee } \\
\text { (external evaluators) : all members } \\
\text { check all aspects (technical, financial, } \\
\text { management, performance indicators } \\
\text {...) plus institutional visit }\end{array}$ & OMFB / Ministry \\
\hline $\mathrm{EE}$ & $\begin{array}{l}\text { Ministry of } \\
\text { Economic } \\
\text { Affairs }\end{array}$ & \begin{tabular}{|l|} 
Competence \\
Centre \\
Programme
\end{tabular} & $\begin{array}{l}\text { EAS (Enterprise Estonia, the } \\
\text { foundation operating under the } \\
\text { responsibility of the Ministry of } \\
\text { Economic Affairs and } \\
\text { Communications) }\end{array}$ & \begin{tabular}{|l|} 
Announcement of public competition \\
of the programme; submission of \\
short proposals; evaluation of short \\
proposals and decisions on the \\
financing of the preparation of full \\
proposals; submission of full \\
proposals; evaluation of full \\
proposals and adoption of final \\
financing decisions; negotiations with \\
the selected competence centres and \\
conclusion of financing agreements
\end{tabular} & EAS Board \\
\hline$\overline{D E}$ & $\mid \mathrm{SI}$ & \begin{tabular}{|l|} 
Nanotechnology \\
Competence \\
Centre \\
Programme
\end{tabular} & ministry and agencies & $\begin{array}{l}\text { proposals are evaluated by a jury of } 9 \\
\text { international experts stemming from } \\
\text { science and industry }\end{array}$ & $\begin{array}{l}\text { Ministry based on recommendations } \\
\text { of the jury }\end{array}$ \\
\hline UK & Technopolis & \begin{tabular}{|l|} 
Partners in \\
Innovation (P II)
\end{tabular} & $\begin{array}{l}\text { Technopolis selectes the appraisers; } \\
\text { try to find good balance of experts }\end{array}$ & \begin{tabular}{|l|}
2 external and 2 internal experts for \\
each proposal; $1-5$ scale with \\
standard deviation; YES/NO criteria \\
is the main selection; not \\
mechanisitic -discussion after \\
numerical ranking leads to change of \\
up to $25 \%$; complete process is \\
anonymous, in the end list of \\
appraisers is published
\end{tabular} & $\begin{array}{l}4 \text { people - } 4 \text { to } 0 \text { stars; } 4 \text { stars no } \\
\text { discussion; } 2-3 \text { stars financial cut off } \\
\text { line; minister final decision }\end{array}$ \\
\hline ES & $\begin{array}{l}\text { Asociacion } \\
\text { Unitec }\end{array}$ & \begin{tabular}{|l} 
PCTI- \\
Cooperacion
\end{tabular} & $\begin{array}{l}\text { The Direction of Technology and } \\
\text { Telecommunications (DTT) of the } \\
\text { Department of Industry, Fishing and } \\
\text { Agriculture; in fact: UET }\end{array}$ & $\begin{array}{l}\text { Bureaucratic test. Detailed set of } \\
\text { evaluation criteria. Scoring system } 0 \\
\text { to } 5 \text { points }\end{array}$ & UET executive committee \\
\hline SL & PMSI-lab & $\begin{array}{l}\text { Industrial } \\
\text { Clusters } \\
\text { Development } \\
\text { Programme } \\
\text { (Toolmakers) }\end{array}$ & Ministry of Economic Affairs & $\begin{array}{l}\text { Formal compliance test, Evaluators = } \\
\text { black box; duration } 60 \text { days; amount } \\
\text { of money not disclosed; }\end{array}$ & Ministry of Economic affairs \\
\hline $\mathrm{IE}$ & SEI & Energy & SEI & $\begin{array}{l}\text { Internal evaluation in SEI against } \\
\text { published evaluation critria. Use of } \\
\text { external experts where judged } \\
\text { appropriate }\end{array}$ & $\begin{array}{l}\text { SEI prior to formal approval by } \\
\text { external committee }\end{array}$ \\
\hline $\mathrm{BE}$ & IIWT & \begin{tabular}{|l|} 
VIS-CIN \\
(Cooperative \\
Innovation \\
Networks)
\end{tabular} & IWT & $\begin{array}{l}\text { Appointement with the project } \\
\text { submittant by IWT-staff, possibility to } \\
\text { make clarifications, discussion with } \\
\text { external referrees, formulation of IWT } \\
\text { advice }\end{array}$ & IWT Board \\
\hline
\end{tabular}




\begin{tabular}{|c|c|c|c|c|c|}
\hline & & & & & \\
\hline Country & Organisation & MAP & \begin{tabular}{|l|}
$\begin{array}{l}\text { 10. Application / evaluation } \\
\text { timetable for evaluation process }\end{array}$ \\
\end{tabular} & $\begin{array}{l}\text { 11. Costs and resources for } \\
\text { evaluation process }\end{array}$ & 12. Selection of evaluators \\
\hline$\overline{A T}$ & TIG & \begin{tabular}{|l|} 
Kplus \\
Competence \\
Centre \\
Programme \\
\end{tabular} & $\begin{array}{l}\text { roughly one year ( } 3 \text { months for short } \\
\text { proposals deadline),.... For starting } \\
\text { a centre between } 4 \text { and } 12 \text { months }\end{array}$ & $\begin{array}{l}0,1 \% \text { of funding, TIG pays a nominal } \\
\text { fee }=500 \text { Euro (more than usually in } \\
\text { basic science) }\end{array}$ & by FWF (Austrian Science Funds) \\
\hline SE & VINNOVA & $\begin{array}{l}\text { Competence } \\
\text { Centre } \\
\text { Programme }\end{array}$ & & \begin{tabular}{|l|} 
external evaluators paid on voluntary \\
basis
\end{tabular} & $\begin{array}{l}\text { chosen from NUTEK network, all } \\
\text { decisions as a group, names known }\end{array}$ \\
\hline $\mathrm{HU}$ & $\begin{array}{l}\text { Ministry of } \\
\text { Education }\end{array}$ & \begin{tabular}{|l|} 
KKK \\
Competence \\
Centre \\
Programme
\end{tabular} & $\begin{array}{l}\text { 1st round: approximately } 2 \text { months } \\
\text { 2nd round: } 3 \text { months }=2 \text { months } \\
\text { evaluation }+1 \text { month for institutional } \\
\text { visits }\end{array}$ & $\begin{array}{l}\text { separated budget at OMFB/Ministry } \\
\text { for evaluation; KKK Committee } \\
\text { members well paid in relation to } \\
\text { normal evaluators; the whole process } \\
\text { was organised by the programme } \\
\text { manager, now the Ministry of } \\
\text { Education is responsible for the } \\
\text { evaluation of proposals }\end{array}$ & \begin{tabular}{|l|} 
Database in OMFB with 10.000 \\
researchers in Hungary; 35 chosen \\
by the Council of OMFB: Main \\
criteria: open minded and trustful \\
people (all Hungarian out of which 2 \\
are foreign citizens but Hungarian \\
born); out of the 11 members 6 are \\
from industry the rest according to \\
quota from university, research \\
institutes, banks, ..; got well paid \\
(EUR 2000 for 5 days working - 20 \\
times more than usual sum for \\
evaluators)
\end{tabular} \\
\hline EE & \begin{tabular}{|l} 
Ministry of \\
Economic \\
Affairs
\end{tabular} & $\begin{array}{l}\text { Competence } \\
\text { Centre } \\
\text { Programme }\end{array}$ & Around half a year. & $\begin{array}{l}\text { Around } 4400000 \text { Estonian Krones } \\
(=281200 \text { Euro })\end{array}$ & by EAS \\
\hline $\mathrm{DE}$ & $|S|$ & \begin{tabular}{|l|} 
Nanotechnology \\
Competence \\
Centre \\
Programme
\end{tabular} & roughly 6 months & not known & by ministry \\
\hline UK & Technopolis & \begin{tabular}{|l|} 
Partners in \\
Innovation (P II)
\end{tabular} & $\begin{array}{l}5 \text { weeks from application to decision; } \\
30 \text { appraisers are paid EUR } 3200 \text { for } \\
5 \text { days work }\end{array}$ & & \\
\hline ES & $\begin{array}{l}\text { Asociacion } \\
\text { Unitec }\end{array}$ & \begin{tabular}{|l} 
PCTI- \\
Cooperacion
\end{tabular} & \begin{tabular}{|l|} 
Two months application period and \\
two months to evaluate and take \\
decisions by officers. One to two \\
months to make public the decisions \\
after check by administrative officers.
\end{tabular} & $\begin{array}{l}\text { Two full dedication experts, Three } \\
\text { part time dedication experts, One } \\
\text { director, one administrative, one } \\
\text { secretary }\end{array}$ & $\begin{array}{l}\text { Experts database. One external } \\
\text { expert per knowledge area. Role } \\
\text { recommendation. Expertise and } \\
\text { independence from proposers is } \\
\text { needed. }\end{array}$ \\
\hline SL & PMSI-lab & \begin{tabular}{|l} 
Industrial \\
Clusters \\
Development \\
Programme \\
(Toolmakers)
\end{tabular} & 60 days & & Black Box \\
\hline IE & SEI & Energy & & & \\
\hline BE & IWT & $\begin{array}{l}\text { VIS-CIN } \\
\text { (Cooperative } \\
\text { Innovation } \\
\text { Networks) }\end{array}$ & $\begin{array}{l}\text { Three months application period and } \\
\text { e evaluation procedure must be done } \\
\text { in } 75 \text { worksdays }\end{array}$ & $\begin{array}{l}\text { Internal costs : } 4 \text { à } 5 \text { VTE advisor } \\
\text { External referrees }\end{array}$ & $\begin{array}{l}\text { A mix of the background of experts is } \\
\text { needed : academic, economist, } \\
\text { innovation mgt, industrial background } \\
\text { Selected from the knowledge base of } \\
\text { IWT }\end{array}$ \\
\hline
\end{tabular}




\begin{tabular}{|c|c|c|c|c|c|}
\hline & & & & & \\
\hline Country & Organisation & MAP & 13.Traps & $\begin{array}{l}\text { 14. Evaluation criteria for the } \\
\text { selection of proposals }\end{array}$ & $\begin{array}{l}\text { 15. Experiences with anonymous I } \\
\text { non-anonymous evaluation }\end{array}$ \\
\hline$\overline{A T}$ & TIG & \begin{tabular}{|l} 
Kplus \\
Competence \\
Centre \\
Programme
\end{tabular} & $\begin{array}{l}\text { communicate often what are the aims } \\
\text { of the programme; loosing balance } \\
\text { between scientific excellent and } \\
\text { management; style of scientific } \\
\text { criticism (very soft) }\end{array}$ & see others & $\begin{array}{l}\text { written reviews are strictly } \\
\text { confidential; names are just known } \\
\text { from the visiting committee }\end{array}$ \\
\hline SE & VINNOVA & \begin{tabular}{|l|} 
Competence \\
Centre \\
Programme
\end{tabular} & $\begin{array}{l}\text { interpretation of criteria not accepted } \\
\text { / understood by some evaluators; } \\
\text { balance of interest }\end{array}$ & $\begin{array}{l}\text { Effect of renewal important, but } \\
\text { difficult to interpret }\end{array}$ & $\begin{array}{l}\text { non anonymous; "principle of } \\
\text { publicity" in all public agencies; } \\
\text { recommendations given by groups, } \\
\text { not individuals; outcome accepted by } \\
\text { research community }\end{array}$ \\
\hline $\mathrm{HU}$ & $\begin{array}{l}\text { Ministry of } \\
\text { Education }\end{array}$ & \begin{tabular}{|l|} 
KKK \\
Competence \\
Centre \\
Programme
\end{tabular} & & $\begin{array}{l}\text { The main principle was the viability of } \\
\text { the centre, but also scientific quality, } \\
\text { the personnel choosen, the director, } \\
\text { the technology innovation, the } \\
\text { reseach and training strategy, the } \\
\text { business plan of the centre, the } \\
\text { utilisation of IPR and the quality } \\
\text { assurance procedures for the } \\
\text { functioning of the centres. }\end{array}$ & $\begin{array}{l}\text { Winners get results, losers only on } \\
\text { request; problems with secrecy } \\
\text { (pharmaceuticals); } \\
\text { Evaluators were public (first time in } \\
\text { Hungary) - increased quality and } \\
\text { responsibility also from the side of } \\
\text { the evaluators; names of evaluators } \\
\text { knwon beforehand }\end{array}$ \\
\hline EE & $\begin{array}{l}\text { Ministry of } \\
\text { Economic } \\
\text { Affairs }\end{array}$ & \begin{tabular}{|l|} 
Competence \\
Centre \\
Programme
\end{tabular} & $?$ & \begin{tabular}{|l|} 
elgibility; objectives and proposed \\
outcomes of the CC; quality of \\
research programme of CCs; quality \\
of application of outcomes of \\
research programme; Development of \\
human resources for the \\
implementation of research \\
programme; CC consortium and \\
organisation structure; work plan and \\
financial plan of CC
\end{tabular} & $?$ \\
\hline $\mathrm{DE}$ & $\mid \mathrm{ISI}$ & \begin{tabular}{|l|} 
Nanotechnology \\
Competence \\
Centre \\
Programme
\end{tabular} & $\begin{array}{l}\text { finding independent but also } \\
\text { accepted experts for the jury }\end{array}$ & \begin{tabular}{|l|} 
scientific performance, cooperation \\
features; existing cooperations / \\
networks; innovative products, \\
procedures, services; new \\
enterprises; education and further \\
training; norms and standards; public \\
relations; long-term perspective of the \\
CCN
\end{tabular} & reviews are strictly confidential \\
\hline UK & Technopolis & \begin{tabular}{|l|} 
Partners in \\
Innovation (P II)
\end{tabular} & $\begin{array}{l}\text { Conflict of interest - agreement; } \\
\text { immediate returning proposal from } \\
\text { project; different department is OK; } \\
\text { not in final discussion } \\
\text { "secret" parts of the proposal - HARD } \\
\text { STAND: if ist so secret - fund it } \\
\text { yourself }\end{array}$ & known to the applicants & \\
\hline ES & $\begin{array}{l}\text { Asociacion } \\
\text { Unitec }\end{array}$ & \begin{tabular}{|l} 
PCTI- \\
Cooperacion
\end{tabular} & \begin{tabular}{|l|} 
Little time period to select/invite/bring \\
in external evaluator --> less \\
expertise than desired to be obtained.
\end{tabular} & $\begin{array}{l}\text { Human ressources, Innovative } \\
\text { Character, suitable methodology, } \\
\text { correct management } \\
\text { eligibilty, scientific quality, } \\
\text { management, exploitation plans; } \\
\text { scores assigned to qualitative criteria }\end{array}$ & always non-anonymous \\
\hline SL & PMSI-lab & \begin{tabular}{|l|} 
Industrial \\
Clusters \\
Development \\
Programme \\
(Toolmakers)
\end{tabular} & & $\begin{array}{l}\text { Eligibility, Scientific quality, socio- } \\
\text { economic aspects, Management, } \\
\text { National strategies implementation } \\
\text { impact }\end{array}$ & anonymous \\
\hline IE & SEI & Energy & & \begin{tabular}{|l|} 
Technical compliance. Relevance to \\
objectives of programme. Project mgt \\
capability. Provision for follow-up and \\
dissemination. Compliance with \\
admin requirements
\end{tabular} & \\
\hline $\mathrm{BE}$ & IWT & \begin{tabular}{|l|} 
VIS-CIN \\
(Cooperative \\
Innovation \\
Networks)
\end{tabular} & \begin{tabular}{|l|} 
People which follow the news of IWT \\
of very well, have advantage on \\
organisation which are new in the \\
field. Projects which are written down \\
very well, are not always the best \\
proposals. The difficulty is take into \\
account a lot of of tacit and informal \\
information in the selection \\
procedure, whilst staying objective \\
and neutral and relying the decision \\
on measureble elements
\end{tabular} & \begin{tabular}{|l|} 
Two main dimension : technical \\
quality of the proposal, innovation \\
and socio-economic potential/ Also \\
considered : track record and overall \\
distribution of money (not to one \\
technology or one sector)
\end{tabular} & Non-anoymous evaluation \\
\hline
\end{tabular}


12.3.4 Proposal Evaluation of MAPs (June 2002)

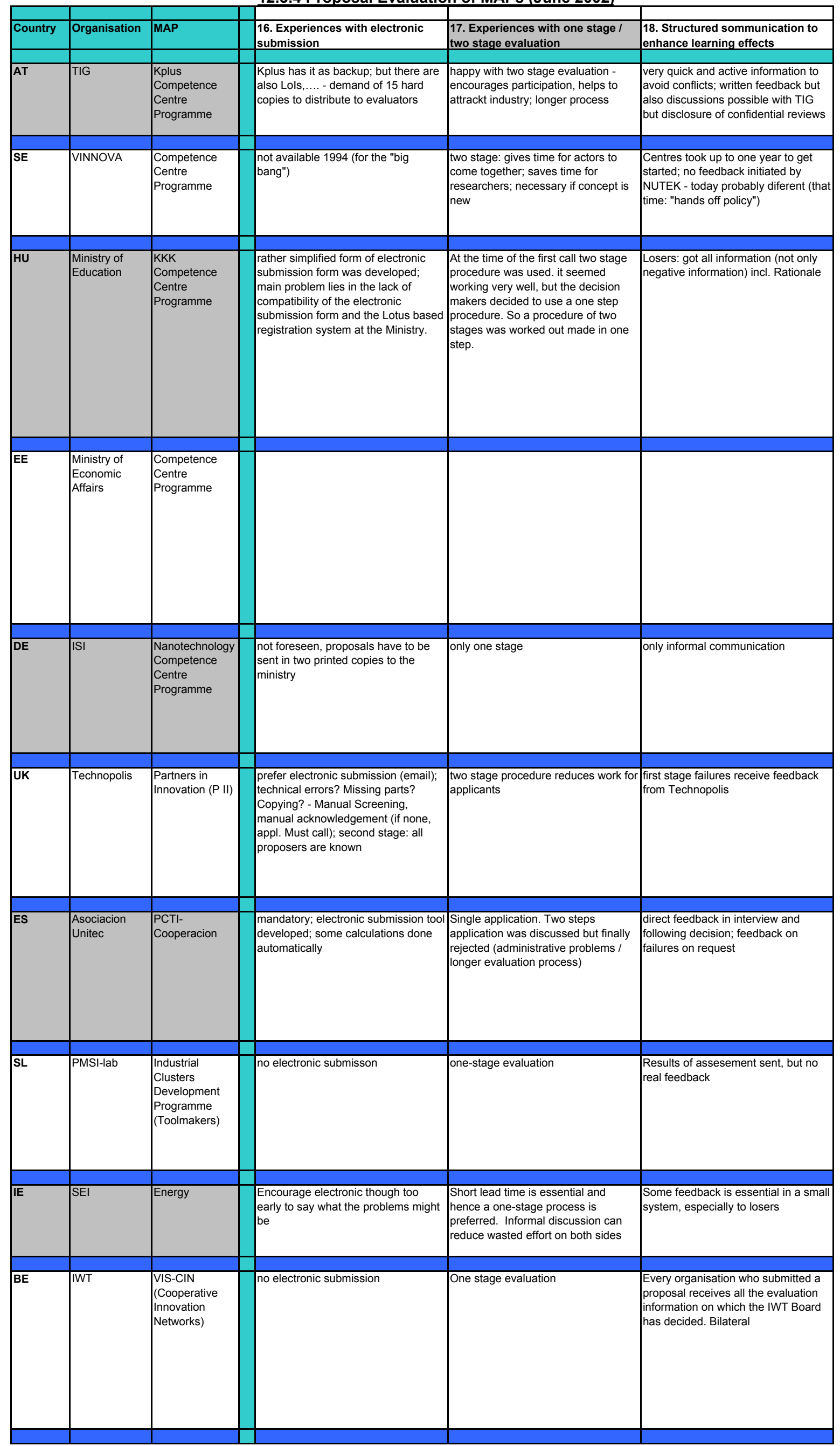




\begin{tabular}{|c|c|c|c|}
\hline & & & \\
\hline Country & Organisation & MAP & 19. Critical success factors \\
\hline AT & TIG & \begin{tabular}{|l} 
Kplus \\
Competence \\
Centre \\
Programme
\end{tabular} & $\begin{array}{l}\text { quality of outcome; even applicants } \\
\text { that are not chosen accept the } \\
\text { outcome as correct and fair }\end{array}$ \\
\hline SE & VINNOVA & \begin{tabular}{|l|} 
Competence \\
Centre \\
Programme
\end{tabular} & $\begin{array}{l}\text { Succesful development of centres, } \\
\text { outcome accepted by research } \\
\text { community; one centre was a failure, } \\
\text { because it was a network between } \\
\text { three universities; new concepts and } \\
\text { criteria }\end{array}$ \\
\hline $\mathrm{HU}$ & $\begin{array}{l}\text { Ministry of } \\
\text { Education }\end{array}$ & \begin{tabular}{|l} 
KKK \\
Competence \\
Centre \\
Programme
\end{tabular} & $\begin{array}{l}5 \text { centers running, } 4 \text { working well; } 73 \\
\text { applicants - only one complaining } \\
\text { about process; } 3 \text { centres were set up } \\
\text { without public money; strength: } \\
\text { transparency, open information; } \\
\text { flexibility with applicants }\end{array}$ \\
\hline EE & \begin{tabular}{|l} 
Ministry of \\
Economic \\
Affairs
\end{tabular} & \begin{tabular}{|l|} 
Competence \\
Centre \\
Programme
\end{tabular} & $\begin{array}{l}\text { satisfaction of applicants; the } \\
\text { selection of experts }\end{array}$ \\
\hline $\mathrm{DE}$ & $|S|$ & \begin{tabular}{|l|} 
Nanotechnology \\
Competence \\
Centre \\
Programme
\end{tabular} & $\begin{array}{l}\text { quality of outcome; acceptance of the } \\
\text { procedure in terms of time/duration, } \\
\text { selected peers, results, transparency }\end{array}$ \\
\hline UK & Technopolis & \begin{tabular}{|l|} 
Partners in \\
Innovation (P II)
\end{tabular} & $\begin{array}{l}\text { Monitoring application numbers, } \\
\text { application satisfaction levels; learn } \\
\text { form others and adapt; cyclicyl } \\
\text { consultation with stakeholders; failed } \\
\text { iniatives: demanding } 10 \% \text { cash (clear } \\
\text { definition! }\end{array}$ \\
\hline ES & $\begin{array}{l}\text { Asociacion } \\
\text { Unitec }\end{array}$ & \begin{tabular}{|l} 
PCTI- \\
Cooperacion
\end{tabular} & $\begin{array}{l}\text { Evaluation guide: weight of criteria; } \\
\text { indicators: value the impact to } \\
\text { improve the process; Strength: direct } \\
\text { interview und use of external experts; } \\
\text { Weakness: administrative process } \\
\text { prior to techn. eval is too long; } \\
\text { Interaction with proposers would } \\
\text { increase quality; Specific contract } \\
\text { proposers and Government }\end{array}$ \\
\hline SL & PMSI-lab & \begin{tabular}{|l|} 
Industrial \\
Clusters \\
Development \\
Programme \\
(Toolmakers)
\end{tabular} & $\begin{array}{l}\text { Evaluation procedures not too } \\
\text { complicated, fast process, fair results; } \\
\text { simple and transparent criteria; fair } \\
\text { play; good ex-post monitoring } \\
\text { system; To be changed: not black } \\
\text { box, procedures too long; best } \\
\text { practice: open systems for applicants } \\
\text { questions }\end{array}$ \\
\hline IE & SEI & Energy & $\begin{array}{l}\text { Perceived fairness. Speed of } \\
\text { processing. Clarity of link with } \\
\text { evaluation criteria. Acceptance by } \\
\text { proposer of result. }\end{array}$ \\
\hline BE & IWT & \begin{tabular}{|l|} 
VIS-CIN \\
(Cooperative \\
Innovation \\
Networks)
\end{tabular} & $\begin{array}{l}\text { Clear information beforehand, Good } \\
\text { preparation of the process } \\
\text { beforehand (no improvisation during } \\
\text { the selection procedure), open } \\
\text { communication towards all the } \\
\text { players involved }\end{array}$ \\
\hline
\end{tabular}


12.3.5 Contract Negotiations (November 2002)

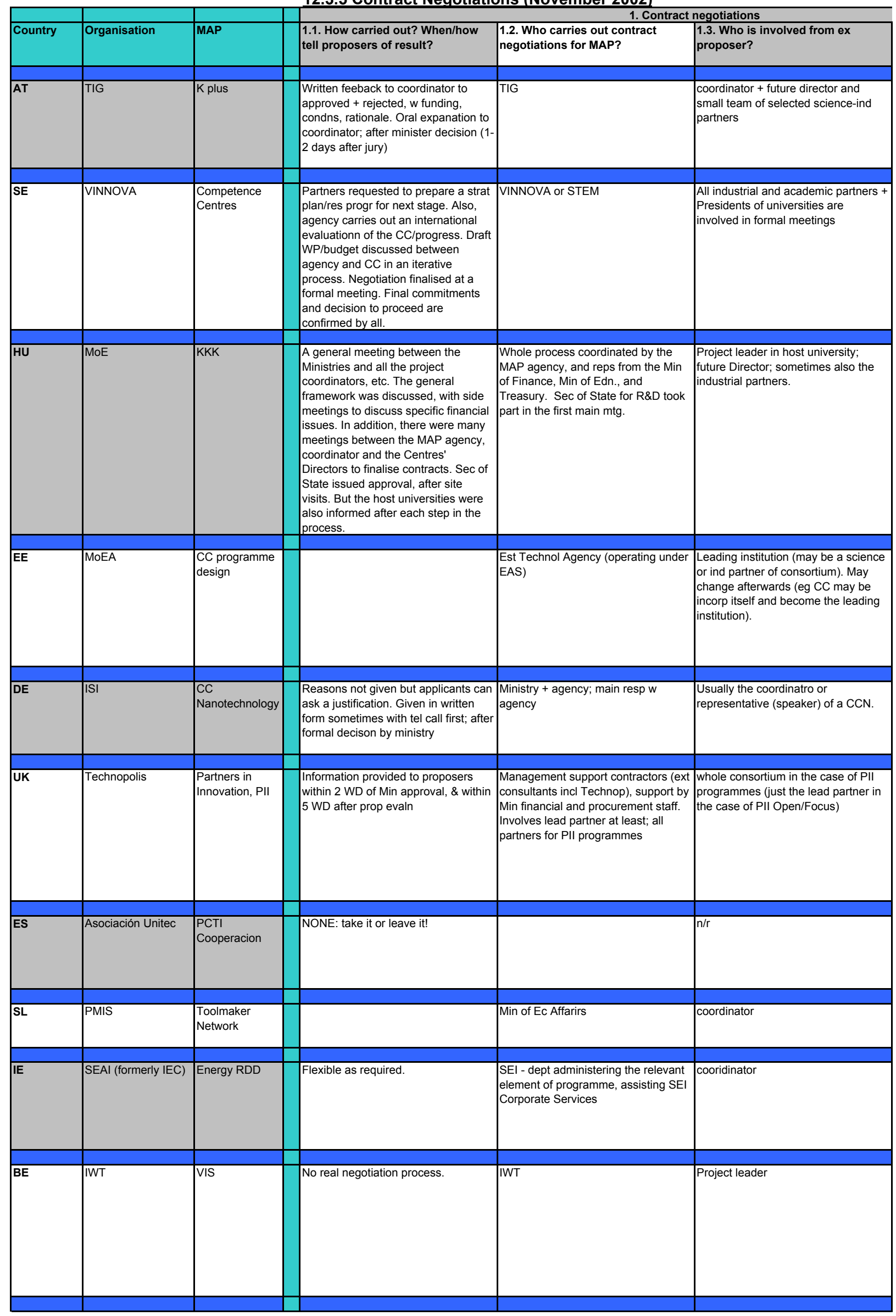




\begin{tabular}{|c|c|c|c|c|c|}
\hline & & & & & \\
\hline Country & Organisation & MAP & 1.4. Form of negotiations? & 2.1. What is the contract like? & $\begin{array}{l}\text { 2.2. Who checks governmental } \\
\text { aid/quota? }\end{array}$ \\
\hline AT & TIG & K plus & $\begin{array}{l}\text { Mix of meetings with coordinator; } \\
\text { presumptive director; small team; + } \\
\text { some with all partnersForm of } \\
\text { negotiations? Mix writing/meetings, } \\
\text { formal/informal }\end{array}$ & $\begin{array}{l}\text { Agreements signed by all partners } \\
\text { based on BP. Standard funding } \\
\text { contract between TIG/Centre. V } \\
\text { Standard (BP) individual contracts } \\
\text { TIG-partners }\end{array}$ & $\begin{array}{l}\text { Specification in Agreement \& Funding } \\
\text { contract. Checked by TIG, Centre, } \\
\text { TIG/evaluation }\end{array}$ \\
\hline SE & VINNOVA & $\begin{array}{l}\text { Competence } \\
\text { Centres }\end{array}$ & $\begin{array}{l}\text { Meetings with coordinator }(\mathrm{CC}) \text { and } \\
\text { finally with whole consortium }\end{array}$ & $\begin{array}{l}\text { A Principal Agreement - incl LT } \\
\text { strategic plan, joint research } \\
\text { programme., budget and success } \\
\text { criteria. More details in paper. }\end{array}$ & $\begin{array}{l}\text { VINNOVA or STEM, before } \\
\text { agreement is signed, and again when } \\
\text { final report has been submitted by } \\
\text { CC. }\end{array}$ \\
\hline HU & MoE & KKK & See 2.1 above. & $\begin{array}{l}\text { A model contract with reference to the } \\
\text { Govt decree. The KKK contract } \\
\text { annexed (agreement between } \\
\text { founding members) and an } \\
\text { agreement on IPR are peculiar to the } \\
\text { KKK programme. }\end{array}$ & $\begin{array}{l}\text { Financial part of the contract is only } \\
\text { checked after the R\&D activities have } \\
\text { been approved (by Min of Edn R\&D } \\
\text { Division). MAP agency checks the } \\
\text { financial elements. }\end{array}$ \\
\hline $\mathrm{EE}$ & MoEA & \begin{tabular}{|l|} 
CC programme \\
design
\end{tabular} & & $\begin{array}{l}\text { Regulates rights and obligations of } \\
\text { EAS and the lead institution. Must be } \\
\text { concluded within } 5 \text { moths after the } \\
\text { financing decision. }\end{array}$ & Ministry of Finance \\
\hline $\mathrm{DE}$ & $\mid \mathrm{SI}$ & \begin{tabular}{|l|} 
CC \\
Nanotechnology
\end{tabular} & $\begin{array}{l}\text { Mix: sometimes meetings, telephone, } \\
\text { written... }\end{array}$ & A standard (not special) type & The agency, but Ministry involved too \\
\hline UK & Technopolis & \begin{tabular}{|l|} 
Partners in \\
Innovation, PII
\end{tabular} & $\begin{array}{l}100 \% \text { meetings for PII Programmes. } \\
33 \% \text { meetings for PII Open/ Focus; } \\
66 \% \text { just written for PII Open/Focus }\end{array}$ & $\begin{array}{l}\text { Standard terms: liability, conduct IPR, } \\
\text { termination, etc. CN moves from } \\
\text { proposal to explicit commitments on } \\
\text { outputs, milestones, resources. }\end{array}$ & $\begin{array}{l}\text { Research Management Contractor, } \\
\text { with queries referred to DTI if } \\
\text { necessary. DTI checks programmes } \\
\text { against state aids. }\end{array}$ \\
\hline ES & Asociación Unitec & $\begin{array}{l}\text { PCTI } \\
\text { Cooperacion }\end{array}$ & $\mathrm{n} / \mathrm{r}$ & $\begin{array}{l}\text { No contract; accepting the money } \\
\text { implies accept of decree detail }\end{array}$ & $\begin{array}{l}\text { The Technology Strategy Unit in the } \\
\text { first place; then governmental } \\
\text { auditors. }\end{array}$ \\
\hline SL & PMIS & $\begin{array}{l}\text { Toolmaker } \\
\text { Network }\end{array}$ & just written form & $\begin{array}{l}\text { Standard contract prepared by } \\
\text { Ministry of Economic Affairs }\end{array}$ & Ministry \\
\hline IE & SEAI (formerly IEC) & Energy RDD & & $\begin{array}{l}\text { A standard SEI contract with WP } \\
\text { annexed. }\end{array}$ & SEI \\
\hline $\mathrm{BE}$ & IWT & VIS & & $\begin{array}{l}\text { Like normal IWT contracts as far as } \\
\text { poss. Diffs include: interaction w } \\
\text { Users Cttee; duty to support further } \\
\text { ec use of res o/p; due diligence in } \\
\text { reln to IPR and confy. }\end{array}$ & $\begin{array}{l}\text { No in-advance quotas; whole thing is } \\
\text { bottom up (GBOU and VIS). Written } \\
\text { evidence of requested final input } \\
\text { must be supplied where relevant. } \\
\text { Overlap/double financing checked } \\
\text { prior to IWT Board decision. }\end{array}$ \\
\hline
\end{tabular}




\begin{tabular}{|c|c|c|c|c|c|}
\hline & & & & & 2. Contract $s$ \\
\hline Country & Organisation & MAP & $\begin{array}{l}\text { 2.3.Integration of proposal } \\
\text { evaluation within work } \\
\text { programme? }\end{array}$ & 2.4. Average length of contract? & 2.5. Funding \\
\hline AT & TIG & K plus & \begin{tabular}{|l|} 
Proposal plans updated with \\
recommendations from eval. \\
Respective plan is part of agreement \\
(2-3 years)
\end{tabular} & 4 years (Agreement/ funding contract & Broken into annual budgets \\
\hline SE & VINNOVA & \begin{tabular}{|l} 
Competence \\
Centres
\end{tabular} & $\begin{array}{l}\text { Results of international evaluation is } \\
\text { an input to the } \mathrm{CC} \text { and negotiations. } \\
\text { Taken into account in planning next } \\
\text { stage. Actions taken are reported at } \\
\text { final confirming meeting and followed } \\
\text { up at next evaluation. }\end{array}$ & 2-3 years/agrmnt. & \\
\hline HU & MoE & KKK & \begin{tabular}{|l|} 
All proposals revised after the \\
evaluation process. A meeting betw \\
the KKK Committee and the Centre \\
Directors discussed all aspects of the \\
proposals. Some industrial partners \\
also involved in this.
\end{tabular} & $\begin{array}{l}3 \text { years, but support should last at } \\
\text { least } 9 \text { years, with repeat contracts. }\end{array}$ & $\begin{array}{l}\text { No fixed \% for payments; depends on } \\
\text { the reporting period in the contract. } \\
\text { Inititial advance } 10 \% \text {; Based on WP, } \\
\text { but payments only after finishing a } \\
\text { working period, which creates } \\
\text { liquidity problems. }\end{array}$ \\
\hline $\mathrm{EE}$ & MoEA & $\begin{array}{l}\text { CC programme } \\
\text { design }\end{array}$ & $\begin{array}{l}\text { WP has to be revised taking } \\
\text { evaluation comments into account, } \\
\text { and this is dealt with during } \\
\text { negotiation. }\end{array}$ & 3 years & $\begin{array}{l}\text { Determined in the Financial } \\
\text { Agreement }\end{array}$ \\
\hline $\mathrm{DE}$ & ISI & $\begin{array}{l}\text { CC } \\
\text { Nanotechnology }\end{array}$ & $\begin{array}{l}\text { Work plan, cost calculation, } \\
\text { consortium etc have to be changed } \\
\text { by applicants }\end{array}$ & Not $>3$ years & $\begin{array}{l}\text { Final payment not }>10 \% \text { of total. } \\
\text { Needs evidence }\end{array}$ \\
\hline UK & Technopolis & \begin{tabular}{|l|} 
Partners in \\
Innovation, PII
\end{tabular} & \begin{tabular}{|l|} 
Applicants are 'invited' to take \\
account of evaluation, but not \\
mandatory. Greater interest from \\
Ministry where funding \% is greater \\
and where priorities are clearer; but \\
not more than $10-20 \%$ influence. If \\
more than this, then re-submission or \\
a competitive tender process is run.
\end{tabular} & $\begin{array}{l}\text { Varies from a few months for small } \\
\text { grants to } 5-10 \text { years }\end{array}$ & $\begin{array}{l}\text { No fixed \% for payments; is based on } \\
\text { work and milestones; initial advance } \\
\text { being considered; finaly payment } \\
\text { normally }>10 \%\end{array}$ \\
\hline ES & Asociación Unitec & $\begin{array}{l}\mathrm{PCTI} \\
\text { Cooperacion }\end{array}$ & $\begin{array}{l}\text { Before, yes. Now consortia are } \\
\text { expected to adopt recommendations } \\
\text { from evaluators }\end{array}$ & 3 years & \\
\hline SL & PMIS & $\begin{array}{l}\text { Toolmaker } \\
\text { Network }\end{array}$ & & 1 year & $\begin{array}{l}\text { Periodic payment is defined in the } \\
\text { contract. }\end{array}$ \\
\hline IE & SEAI (formerly IEC) & Energy RDD & $\begin{array}{l}\text { Modification to WP on basis of } \\
\text { observations from evaluation. }\end{array}$ & $\begin{array}{l}0.5 \text { to } 3 \text { years. Average about } 2 \\
\text { years. }\end{array}$ & $\begin{array}{l}\text { In phases based on milestones in } \\
\text { contract. }\end{array}$ \\
\hline BE & IWT & VIS & $\begin{array}{l}\text { Clear modifications are made on } \\
\text { basis of evaluators' or IWT's analysis, } \\
\text { but with limited scope for } \\
\text { modifications other than re-submit. }\end{array}$ & 4 years & 9 equal instalments \\
\hline
\end{tabular}




\begin{tabular}{|c|c|c|c|c|c|}
\hline & & & pecifications & & \\
\hline Country & Organisation & MAP & 2.6. Freqency of payments? & $\begin{array}{l}\text { 2.7. Project deliverables - in what } \\
\text { form? }\end{array}$ & 2.8. How often are reports due? \\
\hline AT & TIG & K plus & $\begin{array}{l}\text { Quaterly based on budget + progress } \\
\text { (controlled by TIG) }\end{array}$ & $\begin{array}{l}\text { Each Centre has research projects; } \\
\text { deliverables planned at project level } \\
\& \text { incl in agreement }\end{array}$ & $\begin{array}{l}\text { Financial: quarterly; } 1 \text { overall annual } \\
\text { report }\end{array}$ \\
\hline $\mathbf{S E}$ & $\begin{array}{l}\text { VINNOVA } \\
\end{array}$ & \begin{tabular}{|l|} 
Competence \\
Centres
\end{tabular} & \begin{tabular}{|l|} 
Quaterly from VINNOVA to the \\
university. Ind Partners' payments are \\
in proportion with VINNOVA's.
\end{tabular} & $\begin{array}{l}\text { Project results: publ in accordance w } \\
\text { good intern stds. Annual progress } \\
\text { report and final report from CCs. }\end{array}$ & Annually and at end of stage \\
\hline HU & $\mathrm{MoE}$ & KKK & Generally every 6 months. & Scientific activity reports and costs. & $\begin{array}{l}\text { Is defined by applicants and written } \\
\text { into the contract WP. }\end{array}$ \\
\hline $\mathrm{EE}$ & MoEA & $\begin{array}{l}\text { CC programme } \\
\text { design }\end{array}$ & 2/year & $\begin{array}{l}\text { Report formats specified by EAS. } \\
\text { Strong focus on financing. }\end{array}$ & see above \\
\hline $\mathrm{DE}$ & $\mid \mathrm{ISI}$ & \begin{tabular}{|l|} 
CC \\
Nanotechnology
\end{tabular} & Every 3 months to companies & Reports & $\begin{array}{l}\text { Interim reports every } 6 \text { months. } \\
\text { Annual exloitation plan. Final report } 6 \\
\text { months after project completion, incl } \\
\text { an evaluation report }\end{array}$ \\
\hline UK & Technopolis & \begin{tabular}{|l|} 
Partners in \\
Innovation, PII
\end{tabular} & $\begin{array}{l}\text { Open/Focus: depends on milestones. } \\
\text { Programmes: half-yearly based on full } \\
\text { annual performance audit. }\end{array}$ & $\begin{array}{l}\text { All: Admin (tech/fin) reports. Other: } \\
\text { project-specific. }\end{array}$ & $\begin{array}{l}\text { Agreed by DTI/lead partner based on } \\
\text { milestones }\end{array}$ \\
\hline ES & Asociación Unitec & \begin{tabular}{|l|} 
PCTI \\
Cooperacion
\end{tabular} & $1 /$ year & $\begin{array}{l}\text { Progress reports incl cost statements } \\
\& \text { technical reports. Technical } \\
\text { deliverables depending....even large } \\
\text { equipt.; electronic }\end{array}$ & Annually \\
\hline $\mathbf{S L}$ & PMIS & \begin{tabular}{|l} 
Toolmaker \\
Network
\end{tabular} & 30 days after reports received. & $\begin{array}{l}\text { Specific deliverables, plus costs and } \\
\text { finance reports. }\end{array}$ & Every 60 days \\
\hline$\overline{I E}$ & \begin{tabular}{|l} 
SEAI (formerly IEC) \\
\end{tabular} & \begin{tabular}{|l} 
Energy RDD \\
\end{tabular} & & $\begin{array}{l}\text { Outputs as spec in WP; periodic } \\
\text { reports for phase payments; final } \\
\text { report. }\end{array}$ & \\
\hline $\mathrm{BE}$ & IWT & VIS & & $\begin{array}{l}\text { Research output. Quality of l'action } \\
\text { between res grp and users cttee; potl } \\
\text { for use of res o/p (follow-up R\&D for } \\
\text { basic; TT to SMEs for ind). }\end{array}$ & \\
\hline
\end{tabular}




\begin{tabular}{|c|c|c|c|c|c|}
\hline & & & & & 3.1 \\
\hline Country & Organisation & MAP & $\begin{array}{l}\text { 2.9. Amendments: How are they } \\
\text { dealt with? What restrictions }\end{array}$ & 2.10. Join/leave consortium? & 3.1. Who owns the results? \\
\hline AT & TIG & K plus & $\begin{array}{l}\text { Budget/res plan updated on annual } \\
\text { basis. Budget checked by TIG; res } \\
\text { plan: Centre Board. Other amend: } \\
\text { letter to TIG; Board inv. }\end{array}$ & Rules stipulated in agreement & $\begin{array}{l}\text { Centre owns results and grants } \\
\text { access to use and comm of results }\end{array}$ \\
\hline SE & VINNOVA & \begin{tabular}{|l} 
Competence \\
Centres
\end{tabular} & $\begin{array}{l}\text { Board of CC should keep partners } \\
\text { informed of any possible delays, etc. }\end{array}$ & \begin{tabular}{|l|} 
If a new Ind Partner (these are \\
encouraged), a Connection \\
Agreement is signed by new partner \\
and all ex partners. Withdrawal \\
possible with 6 months written notice.
\end{tabular} & \begin{tabular}{|l|} 
The originator (researcher in HE; co \\
in industry) owns the results. But \\
generally now an Incorporeal Rights \\
Agreement regulates rights and fees, \\
though Pr Agreement grants all \\
Industrial Partners a non-excl comml \\
right of uses.
\end{tabular} \\
\hline $\mathrm{HU}$ & MoE & KKK & \begin{tabular}{|l|} 
Financial tables may be revised each \\
year up to mid-February. \\
Amendments to contract for R\&D \\
activities are longer and more difficult \\
anf there must be a very good \\
reason.
\end{tabular} & $\mid \begin{array}{l}\text { KKK contract (between members) has } \\
\text { special provisions for this. If a partner } \\
\text { leaves, univ must find another } \\
\text { partner(s) to make up the money. A } \\
\text { joining partner may join as a founding } \\
\text { partner or a supporting member. May } \\
\text { be a special fund set up by Ministry to } \\
\text { attract new founding members. }\end{array}$ & $\begin{array}{l}\text { The Centres. But utilisation and } \\
\text { commercialisation depend on the } \\
\text { agreements between members; these } \\
\text { can be very sophisticated. }\end{array}$ \\
\hline EE & MoEA & \begin{tabular}{|l|} 
CC programme \\
design
\end{tabular} & $\begin{array}{l}\text { Cost modifications may be included in } \\
\text { the reports. Must not exceed res prog } \\
\text { overall budget. Modifications } \\
\text { exceeding } 10 \% \text { of total budget must } \\
\text { be approved by Programme Steering } \\
\text { Committee }\end{array}$ & $\begin{array}{l}\text { Is covered in the Consortium Agrmnt } \\
\text { annexed to Final Agreement. }\end{array}$ & $\begin{array}{l}\text { Depends on what has been specified } \\
\text { in the Consortium Agreement. When } \\
\text { results are achieved, the partners } \\
\text { must immediately start to apply for } \\
\text { IPR protection based on the } \\
\text { Consortium Agreement - or else make } \\
\text { results publicly available. }\end{array}$ \\
\hline $\mathrm{DE}$ & $\mid \mathrm{ISI}$ & \begin{tabular}{|l|} 
CC \\
Nanotechnology
\end{tabular} & \begin{tabular}{|l|} 
Depends on size and scope of the \\
amendments; Costs may not increase
\end{tabular} & $\begin{array}{l}\text { No problems, since no formal } \\
\text { obligations in advance }\end{array}$ & $\begin{array}{l}\text { The applicants; they have to apply for } \\
\text { property rights }\end{array}$ \\
\hline UK & Technopolis & \begin{tabular}{|l} 
Partners in \\
Innovation, PII
\end{tabular} & \begin{tabular}{|l|} 
Timing, technical and financial \\
amendments permitted - even \\
increases in funding if benefits exist. \\
Decisions left to mgt contractor based \\
on reasonableness and \\
consequences of denying.
\end{tabular} & $\begin{array}{l}\text { With } 1 \text { mths notice in case of } \\
\text { acquisitions, mergers, loss of key } \\
\text { staff. DTI can terminate for } \\
\text { misconduct etc., or at any time w } 3 \\
\text { mths notice. }\end{array}$ & $\begin{array}{l}\text { Contractor, but Crown has unlimited } \\
\text { rights to use results, and control over } \\
\text { publication of results and further } \\
\text { commercialisation }\end{array}$ \\
\hline ES & Asociación Unitec & \begin{tabular}{|l}
$\mathrm{PCTI}$ \\
Cooperacion
\end{tabular} & $\begin{array}{l}\text { Annually. Including modifications for } \\
\text { next year's WP }\end{array}$ & $\begin{array}{l}\text { Yes, with approval from evaluators } \\
\text { (not a problem because annual } \\
\text { 'contracts') }\end{array}$ & Private property; partners \\
\hline $\mathbf{S L}$ & PMIS & $\begin{array}{l}\text { Toolmaker } \\
\text { Network }\end{array}$ & No amendments & No restrictions & \\
\hline $\mathrm{IE}$ & SEAI (formerly IEC) & Energy RDD & \begin{tabular}{|l|} 
As flexibly as possible. Minor \\
amendments may be confirmed by \\
exchange of letters. Generally not an \\
increase in overall funding.
\end{tabular} & By written agreement to leave. & \begin{tabular}{|l|} 
Research project participants. \\
Attempt to maintain balance between \\
need to protect IP and desire to \\
publish. SEI will help partners in \\
managing protection of IP with \\
commercial potential.
\end{tabular} \\
\hline $\mathrm{BE}$ & IWT & VIS & $\begin{array}{l}\text { Written request from project leader, } \\
\text { and formal letter from IWT's } \\
\text { management. Minimum, as max } \\
\text { flexibility and emphasise on } \\
\text { performance are the keys. }\end{array}$ & $\begin{array}{l}\text { Maximum flexibility to add additional } \\
\text { users to a running project. }\end{array}$ & $\begin{array}{l}\text { Research consortium. Arrangements } \\
\text { must be spec in Consort Agrmnt if }>1 \\
\text { organisation. Users (companies) don't } \\
\text { automatically acquire rights. Balance } \\
\text { betw protection and dissemn is the } \\
\text { aim, in consensus with IWT and univ. } \\
\text { Increase professionalism is an aim. }\end{array}$ \\
\hline & & & & & \\
\hline
\end{tabular}




\begin{tabular}{|c|c|c|c|c|c|}
\hline & & & PR & \multicolumn{2}{|c|}{ 4. Auditing } \\
\hline Country & Organisation & MAP & 3.2. What about dissemination? & 4.1. Financial Audit: How/when? & $\begin{array}{l}\text { 4.2. Technical verification: How } \\
\text { /when? }\end{array}$ \\
\hline AT & TIG & K plus & & $\begin{array}{l}\text { Quaterly financial report provides } \\
\text { basis for funding \& monitor }\end{array}$ & $\begin{array}{l}\text { Prime responsibility with Centre; } \\
\text { External verification by expert peers } \\
\text { during } 4 \text { years evaluation }\end{array}$ \\
\hline SE & VINNOVA & $\begin{array}{l}\text { Competence } \\
\text { Centres }\end{array}$ & $\begin{array}{l}\text { No additionall requests but most CCs } \\
\text { arrange open seminars/workshops. }\end{array}$ & $\begin{array}{l}\text { VINNOVA and/or Ind Partners may } \\
\text { appoint an auditor - only used very } \\
\text { occasionally but can be and are. }\end{array}$ & Regularly by international teams \\
\hline HU & MoE & KKK & $\begin{array}{l}\text { Scientific seminars (at different } \\
\text { intervals) where PhD students } \\
\text { present results. Sometimes with } \\
\text { international partners present; weekly } \\
\text { seminars - only professional attend. } \\
\text { All centres have websites and publish } \\
\text { their results, with open and closed } \\
\text { sections. }\end{array}$ & $\begin{array}{l}\text { Annual report includes a financial } \\
\text { report audited independently. }\end{array}$ & $\begin{array}{l}\text { Annual activity report; Contains a } \\
\text { comparison with previous years, and } \\
\text { plans for the next year. Self- } \\
\text { assessment. }\end{array}$ \\
\hline $\mathrm{EE}$ & MoEA & $\begin{array}{l}\text { CC programme } \\
\text { design }\end{array}$ & & \begin{tabular}{|l|} 
All annual reports must be audited. \\
REps of EAS and EAS-appointed \\
auditors must have free access to a/c \\
admin system plus rel documents.
\end{tabular} & $\begin{array}{l}\text { As part of process of approving half- } \\
\text { yearly and annual reports by } \\
\text { Programme Steering Committee. }\end{array}$ \\
\hline $\mathrm{DE}$ & $\mid \mathrm{ISI}$ & \begin{tabular}{|l|} 
CC \\
Nanotechnology
\end{tabular} & Depends on confidentiality & TBC & TBC \\
\hline UK & Technopolis & \begin{tabular}{|l|} 
Partners in \\
Innovation, PII
\end{tabular} & $\begin{array}{l}\text { Usually TT and dissemn activs } \\
\text { involved, on 'research diffusion' logic. }\end{array}$ & $\begin{array}{l}\text { Random audit - about } 10 \% \text { of } \\
\text { contractors visited annually }\end{array}$ & $\begin{array}{l}\text { Used in the past, but considered too } \\
\text { costly for any added value. }\end{array}$ \\
\hline ES & Asociación Unitec & \begin{tabular}{|l|} 
PCTI \\
Cooperacion
\end{tabular} & $\begin{array}{l}\text { No formal but normally requested by } \\
\text { evaluators/reviewers }\end{array}$ & Never so far & $\begin{array}{l}\text { Yes, and including a superficial } \\
\text { financial audit; at least yearly }\end{array}$ \\
\hline $\mathbf{S L}$ & PMIS & \begin{tabular}{|l} 
Toolmaker \\
Network
\end{tabular} & $\begin{array}{l}\text { Public presentations and press } \\
\text { conferences. }\end{array}$ & $\begin{array}{l}\text { Each project report is audited by Mins } \\
\text { of Ec Affairs and of Finance. }\end{array}$ & $\begin{array}{l}\text { yes, by Min of Ec Affairs; before } \\
\text { contract and after each project report }\end{array}$ \\
\hline IE & SEAI (formerly IEC) & Energy RDD & $\begin{array}{l}\text { Consortium must work with SEl to } \\
\text { maximise exploitation via appropriate } \\
\text { dissemination }\end{array}$ & At discretion of SEI & $\begin{array}{l}\text { As part of verifying and evaluating } \\
\text { project reports at milestones and at } \\
\text { end }\end{array}$ \\
\hline $\mathrm{BE}$ & IWT & VIS & & $\begin{array}{l}\text { Verification of ann fin report. } \\
\text { Straightfwd unless there are negative } \\
\text { indications. }\end{array}$ & $\begin{array}{l}\text { Mainly via participation of IWTs } \\
\text { advisor in user committee meetings. } \\
\text { Closer review of annual technical } \\
\text { report. Mid-term eval after } 2 \text { years } \\
\text { focuses on key deliverables. }\end{array}$ \\
\hline
\end{tabular}




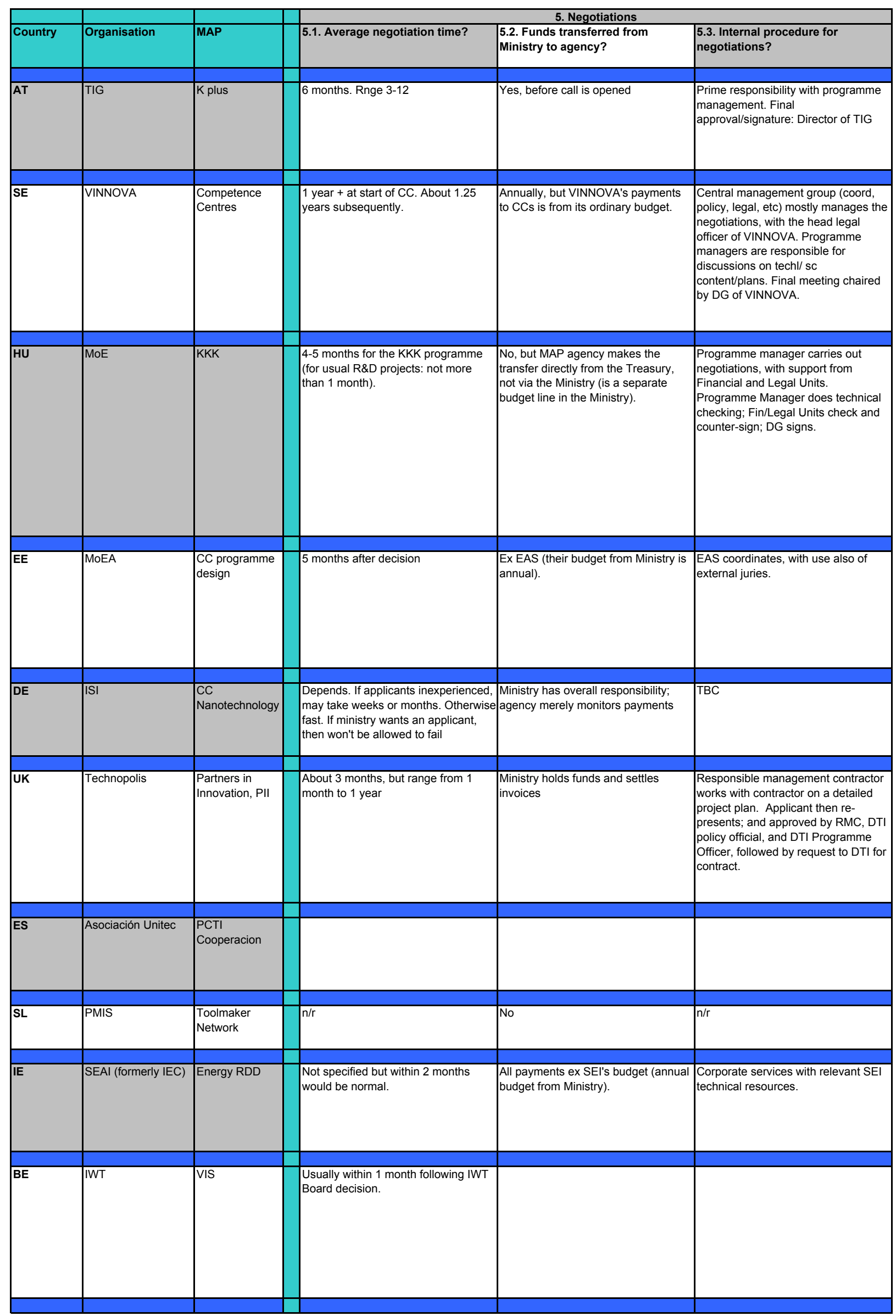




\begin{tabular}{|c|c|c|c|c|c|}
\hline & & & & & 6. Resume \\
\hline Country & Organisation & MAP & 6.1. What worked well? & 6.2. What see as Best Practice? & 6.3. What didn't work? Traps? \\
\hline AT & TIG & K plus & $\begin{array}{l}\text { Social element of negotiation } \\
\text { practice; Agreement as basis for LT } \\
\text { cooperation works well. Centres can } \\
\text { be used to encourage traditional } \\
\text { organisations to learn. }\end{array}$ & $\begin{array}{l}\text { Taking strong positions but remaining } \\
\text { flexible and open. }\end{array}$ & $\begin{array}{l}\text { Lack of info on (eg) LT effects of } \\
\text { different IPR rules. Little influence on } \\
\text { negotiation duruation. Social dynamic } \\
\text { creates undue emphasise on some } \\
\text { issues eg IPR. Flexibility can lead to } \\
\text { sub-optimal outcomes. }\end{array}$ \\
\hline SE & VINNOVA & $\begin{array}{l}\text { Competence } \\
\text { Centres }\end{array}$ & $\begin{array}{l}\text { Input to negotiations from the } \\
\text { international evaluations has been } \\
\text { very successful. Request that CCs } \\
\text { take evaluation comments on board } \\
\text { directly into their plans works well. } \\
\text { Iterative/soft negotion process has } \\
\text { been good where any doubts exist. }\end{array}$ & $\begin{array}{l}\text { A formal agreement, signed by all } \\
\text { (especially the industrial Partners). } \\
\text { Negotiation process that creates } \\
\text { discussion about all issues incl IPR. }\end{array}$ & $\begin{array}{l}\text { Process can be very long drawn-out, } \\
\text { esp if agrmnt not signed immediately } \\
\text { by the Ind Partners. }\end{array}$ \\
\hline HU & MoE & KKK & $\begin{array}{l}\text { Involvement of industrial partners. } \\
\text { Involvement of the KKK Committee } \\
\text { members. }\end{array}$ & $\begin{array}{l}\text { Clear rules for CNs. Existence of a } \\
\text { model contract. }\end{array}$ & $\begin{array}{l}\text { Involvement of the Ministries. } \\
\text { Excessive change in structures, } \\
\text { responsibilities, etc., at time of } \\
\text { implementation. }\end{array}$ \\
\hline $\mathrm{EE}$ & MoEA & $\begin{array}{l}\text { CC programme } \\
\text { design }\end{array}$ & Too soon to say. & & \\
\hline $\mathrm{DE}$ & $\mid \mathrm{ISI}$ & \begin{tabular}{|l|} 
CC \\
Nanotechnology
\end{tabular} & TBC & TBC & TBC \\
\hline UK & Technopolis & $\begin{array}{l}\text { Partners in } \\
\text { Innovation, PII }\end{array}$ & $\begin{array}{l}\text { All works well, on basis of balance } \\
\text { between bureaucracy and application } \\
\text { of human intelligence }\end{array}$ & $\begin{array}{l}\text { Intro of RMCs has worked well. } \\
\text { Switch to milestone-based } \\
\text { reporting/management has helped } \\
\text { control, goal-orientation, and } \\
\text { enforceability. }\end{array}$ & $\begin{array}{l}\text { Closely prescribed rules, eg funding. } \\
\text { Introduction of Steering Committees - } \\
\text { led to inflation, and in-kind } \\
\text { contributions without real } \\
\text { engagement of stakeholders. }\end{array}$ \\
\hline ES & Asociación Unitec & $\begin{array}{l}\text { PCTI } \\
\text { Cooperacion }\end{array}$ & & & \\
\hline SL & PMIS & \begin{tabular}{|l} 
Toolmaker \\
Network
\end{tabular} & & Ability to negotiate contracts & \\
\hline IE & SEAI (formerly IEC) & Energy RDD & $\begin{array}{l}\text { Too soon to provide lessons, either } \\
\text { positive or negative. }\end{array}$ & & \\
\hline $\mathrm{BE}$ & IWT & VIS & \begin{tabular}{|l|} 
Interactive process; positive \\
emphasise on exploitation of \\
research results. Presence of \\
networks / assns. Existence of \\
champions. Adequate \\
communication, flexibility/openness. \\
Alignment of industrial and acadamic \\
interests with mutual respect.
\end{tabular} & & Cult differences; lack of trust \\
\hline
\end{tabular}




\begin{tabular}{|c|c|c|c|c|}
\hline Country & Organisation & MAP & $\begin{array}{l}\text { 6.4. Recommendations for } \\
\text { MAPpers? }\end{array}$ & 6.5. What's MAP-specific? \\
\hline AT & TIG & K plus & $\begin{array}{l}\text { "Interest" vs "Positions". Put a time } \\
\text { limit on negn process }\end{array}$ & $\begin{array}{l}\text { Negotiation of agreements. Less } \\
\text { standardisation possible. Special } \\
\text { roles for agencies, responsible } \\
\text { leaders, \& CC directors }\end{array}$ \\
\hline & & & & \\
\hline SE & VINNOVA & \begin{tabular}{|l} 
Competence \\
Centres
\end{tabular} & $\begin{array}{l}\text { Ensure that ALL performing actors } \\
\text { are really committed to the } \\
\text { cooperation effort. Have formal } \\
\text { agreements signed by all partners. }\end{array}$ & $\begin{array}{l}\text { The formal agreement signed by all } \\
\text { (is motiv by Multi-actor aspect). The } \\
\text { iterative negotiation process is based } \\
\text { on a LT/mutual learning process, } \\
\text { which is specific to MAPs. }\end{array}$ \\
\hline $\mathrm{HU}$ & MoE & KKK & & $\begin{array}{l}\text { Evaluation (KKK) Committee plays } \\
\text { role of facilitator. A more complex } \\
\text { negotiation process. Special contract } \\
\text { format. }\end{array}$ \\
\hline $\mathrm{EE}$ & MoEA & $\begin{array}{l}\text { CC programme } \\
\text { design }\end{array}$ & & \\
\hline$\overline{D E}$ & $\mid \mathrm{ISI}$ & $\begin{array}{l}\text { CC } \\
\text { Nanotechnology }\end{array}$ & TBC & TBC \\
\hline UK & Technopolis & $\begin{array}{l}\text { Partners in } \\
\text { Innovation, PII }\end{array}$ & $\begin{array}{l}\text { CNs should be proportional to size/ } \\
\text { complexity of government funding. } \\
\text { Seek win-win. Avoid autocracy. Don't } \\
\text { micro-manage. }\end{array}$ & $\begin{array}{l}\text { Need to consider each step. High } \\
\text { level of discussion/ consultation. } \\
\text { Highly professional administration }\end{array}$ \\
\hline ES & Asociación Unitec & \begin{tabular}{|l} 
PCTI \\
Cooperacion
\end{tabular} & & \\
\hline $\mathbf{S L}$ & PMIS & $\begin{array}{l}\text { Toolmaker } \\
\text { Network }\end{array}$ & & \\
\hline IE & SEAI (formerly IEC) & Energy RDD & & \\
\hline$\overline{B E}$ & IWT & VIS & $\begin{array}{l}\text { Good balance betw knowledge protn } \\
\text { and dissemn. Balance betw internl } \\
\text { and ec return to region. Balance betw } \\
\text { tf to ex cos vs attn to genberating } \\
\text { spin-off opportunities. Balance betw } \\
\text { need for open/flex approach vs desire } \\
\text { for clarity re things like IPR }\end{array}$ & $\begin{array}{l}\text { Such programmes have to be } \\
\text { designed in the absence of } \\
\text { substantial insights from ex post } \\
\text { evaluations. In MAPs, the significant } \\
\text { o/ps may also be the ones difficult to } \\
\text { quantify. }\end{array}$ \\
\hline
\end{tabular}


12.3.6. Evaluation and Monitoring of MAPs (June / September 2003)

\begin{tabular}{|c|c|c|c|c|}
\hline & & & & \\
\hline Country & Organisation & MAP & 1. Monitoring & 1.1. In which form are project deliverables required \\
\hline AT & TIG & K plus & $\begin{array}{l}\text { A monitoring system which includes financial and output- } \\
\text { related data is in operation }\end{array}$ & $\begin{array}{l}\text { Annual report to TIG and the general public; Quarterly cost } \\
\text { statements, mainly financial but also including progress of } \\
\text { projects to funding agencies; Publications and patents (twice a } \\
\text { year, submitted together with financial report) }\end{array}$ \\
\hline SE & VINNOVA & \begin{tabular}{|l} 
Competence \\
Centres \\
Programme
\end{tabular} & $\begin{array}{l}\text { The system for monitoring of centres (= projects) is modest } \\
\text { with a deliberate principle to decentralize responsibilities to } \\
\text { Centre Boards. }\end{array}$ & Centre progress reports incl cost statements \\
\hline HU & $\mathrm{MoE}$ & KKK & & $\begin{array}{l}\text { Reports: "Scientific Report" + "Financial Repoprt". The } \\
\text { Financial Report is the cost statement. Collection of data: } \\
\text { continuous from the time of applications until the end of a } \\
\text { project and is some cases after heving finished the project. }\end{array}$ \\
\hline $\mathrm{EE}$ & MoEA & $\begin{array}{l}\text { CC programme } \\
\text { design }\end{array}$ & $\begin{array}{l}\text { Everything described here is a plan, because we have no } \\
\text { established any CCs yet }\end{array}$ & \begin{tabular}{|l|} 
1. report about CCs activities and comparison with planned \\
and achieved results. 2 . financial statements for last 6 months
\end{tabular} \\
\hline DE & |SI & $\begin{array}{l}\text { CC } \\
\text { Nanotechnology }\end{array}$ & & $\begin{array}{l}\text { Different kind of reports have to be devlivered: an interim } \\
\text { report (every half year), an annual exploitation plan, a final } \\
\text { report (six months after the project is finished), including a } \\
\text { short summary and a so-called "Erfolgskontrollbericht" } \\
\text { (evaluation report) }\end{array}$ \\
\hline UK & Technopolis & $\begin{array}{l}\text { Partners in } \\
\text { Innovation, PII }\end{array}$ & & $\begin{array}{l}\text { Progress statement, cost statements, copies of any intermediat } \\
\text { outputs }\end{array}$ \\
\hline ES & $\begin{array}{l}\text { Asociación } \\
\text { Unitec }\end{array}$ & $\begin{array}{l}\text { PCTI } \\
\text { Cooperacion }\end{array}$ & $\begin{array}{l}\text { Based on annual reviews of work done in previous period and } \\
\text { on updated workprogramme. Monitoring of projects is } \\
\text { performed by internal officers assisted by administrative } \\
\text { personnel }\end{array}$ & $\begin{array}{l}\text { Monitoring Reports in pre-established formats and electronic } \\
\text { form. Technical deliverables in Consortium decided forms. } \\
\text { Year cost statements }\end{array}$ \\
\hline SL & PMIS & $\begin{array}{l}\text { Toolmaker } \\
\text { Network }\end{array}$ & & reports, cost statements \\
\hline IE & $\begin{array}{l}\text { SEAl (formerly } \\
\text { IEC) }\end{array}$ & Energy RDD & & Reports, cost statements \\
\hline $\mathrm{BE}$ & IWT & VIS & & $\begin{array}{l}\text { The project partners have to submit a scorecard every three } \\
\text { months. After } 20 \text { months there is needed a first summary } \\
\text { report. At the end of the project a thorough end report with } \\
\text { evaluation and feedback from the customer of the project } \\
\text { included. Financial statement are due every year. We ask also } \\
\text { the annual report of each organisation. }\end{array}$ \\
\hline
\end{tabular}




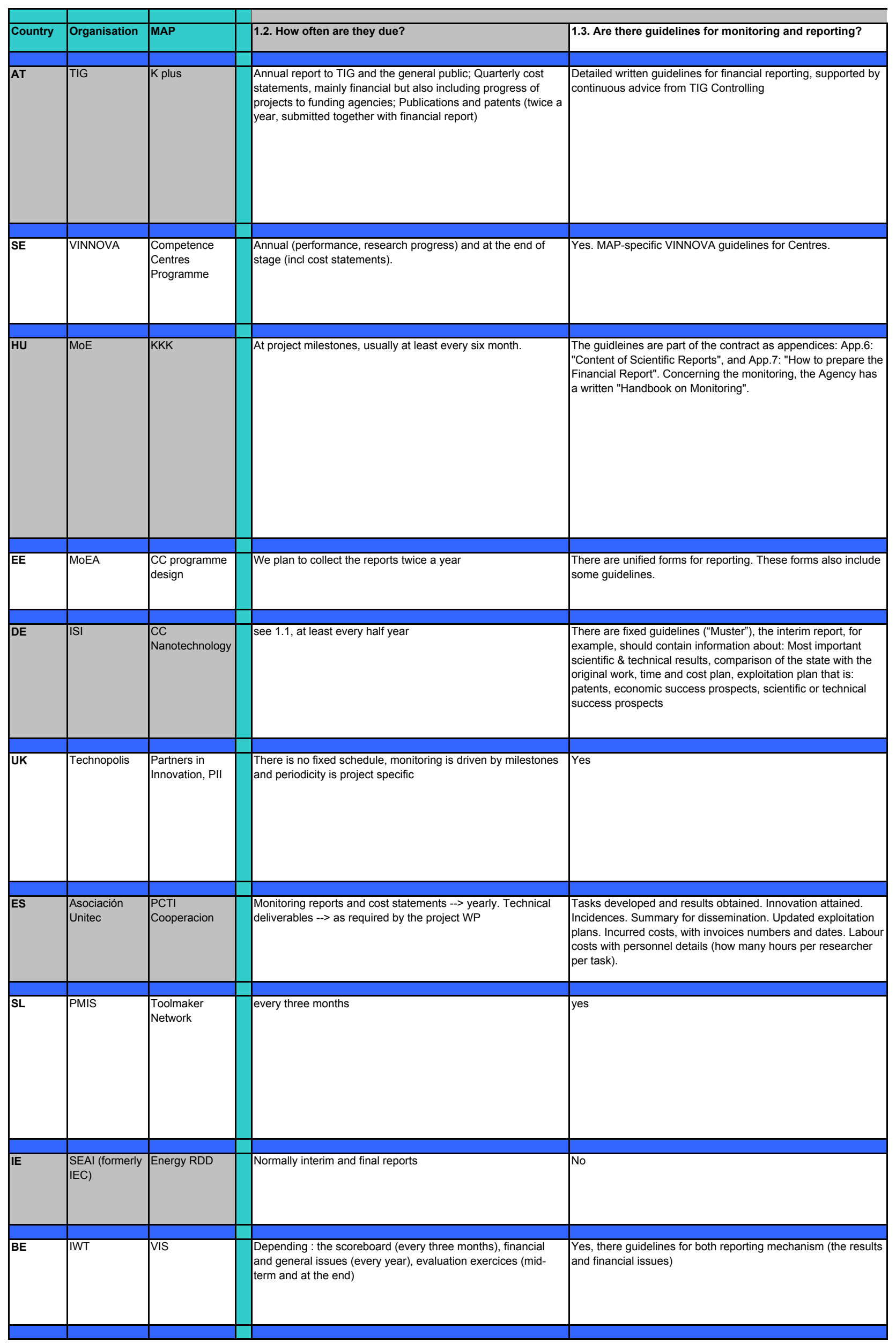




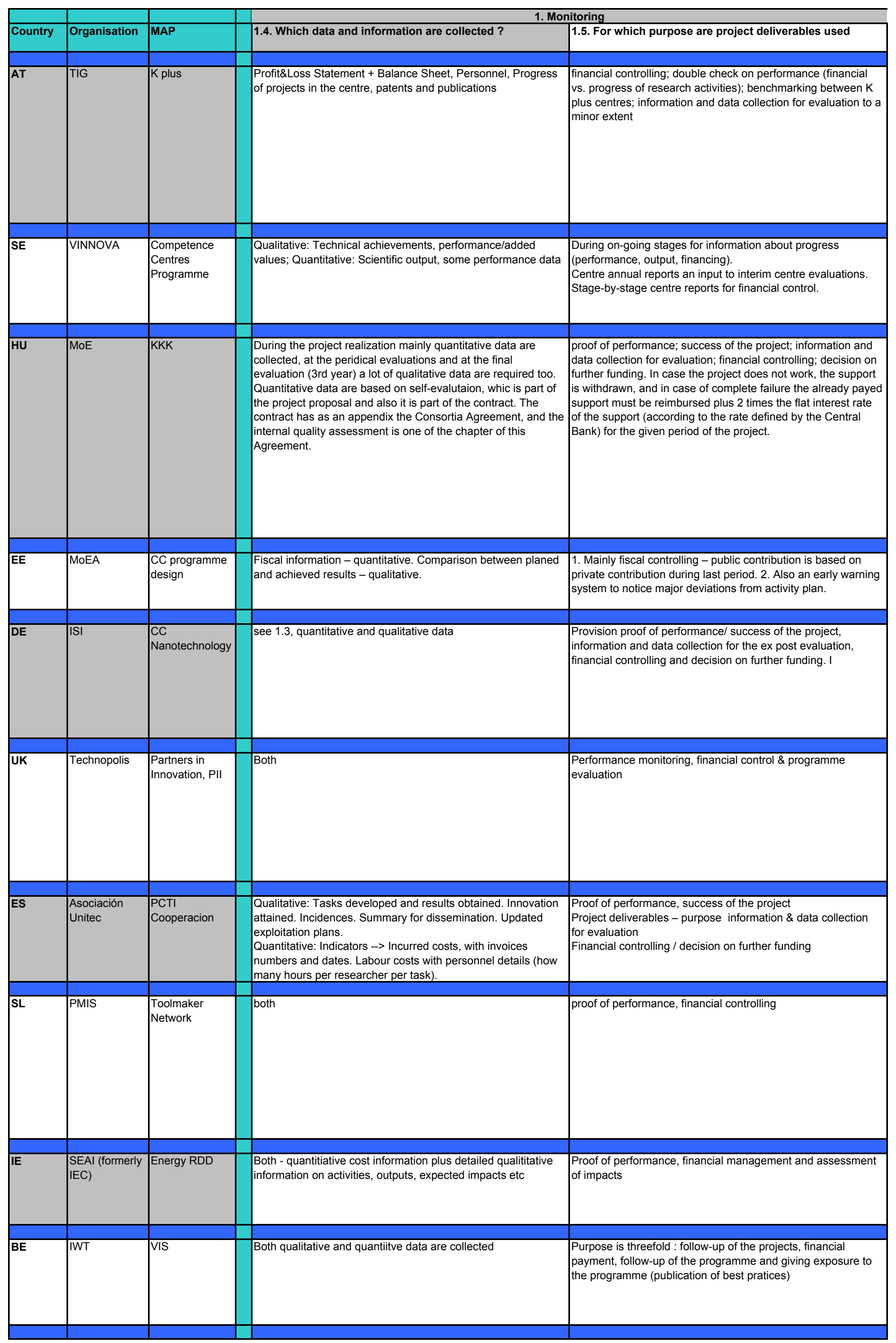




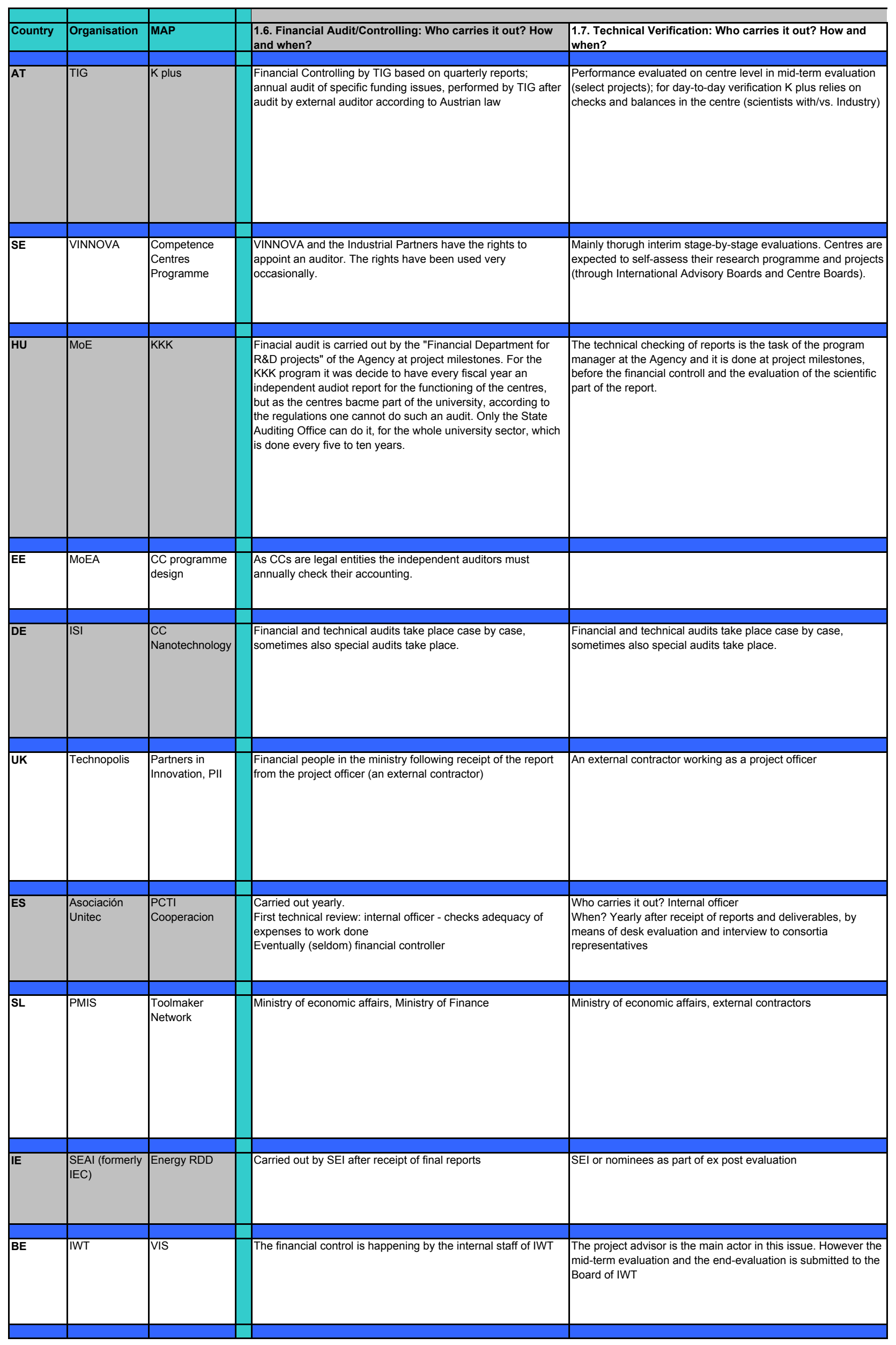




\begin{tabular}{|c|c|c|c|c|}
\hline & & & & \\
\hline Country & Organisation & MAP & 1.8. What are the strengths of your monitoring system? & 1.9. What are the weaknesses of your monitoring system? \\
\hline AT & TIG & K plus & $\begin{array}{l}\text { Monitoring provides sound basis for funding; Controlling effort } \\
\text { is high but arguable with amount of funding; controlling } \\
\text { activities also have a strong educational effect (lack of } \\
\text { controlling competence in universities and subsequently in the } \\
\text { centres); Good but not central input for project evaluation } \\
\text { Participation in boards provides important soft information that } \\
\text { would be impossible to obtain otherwise }\end{array}$ & $\begin{array}{l}\text { Amount of data and information: financial monitoring has } \\
\text { reached high level of sophistication; adaptations may be } \\
\text { considered in the near future }\end{array}$ \\
\hline SE & VINNOVA & \begin{tabular}{|l|} 
Competence \\
Centres \\
Programme
\end{tabular} & $\begin{array}{l}\text { The modest ambitions in our system mean low risk for } \\
\text { monitoring fatigue. The Centres themselves have gradually } \\
\text { realized the value of monitoring data and appreciate the } \\
\text { interim evaluations! }\end{array}$ & $\begin{array}{l}\text { Aggregated programme information (performance, output) not } \\
\text { available until recently. }\end{array}$ \\
\hline & & & 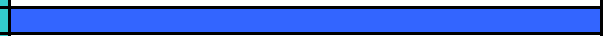 & \\
\hline $\mathrm{HU}$ & MoE & KKK & $\begin{array}{l}\text { The Agency collects a lot of data concerning the projects, both } \\
\text { technical (financial and qualitative for the progress of the } \\
\text { projects) and also data concerning the policy or the rational for } \\
\text { the program. But this later has been used rarely until now. As } \\
\text { there severity measures at national level (less money available } \\
\text { than planned), this later will become more and more important: } \\
\text { impact analysis, etc. }\end{array}$ & $\begin{array}{l}\text { It is too administrative, it is a real burden for the applicants. } \\
\text { The same for the Agency, over complicated administrative } \\
\text { steps, which makes the agecy's work rather non-efficient from } \\
\text { the outside world. (About } 50 \% \text { of the available fund are } \\
\text { disbursed yearly! }\end{array}$ \\
\hline EE & MoEA & $\begin{array}{l}\text { CC programme } \\
\text { design }\end{array}$ & Haven't tried it yet. & Haven't tried it yet. \\
\hline $\mathrm{DE}$ & $\mid \mathrm{SI}$ & $\begin{array}{l}\text { CC } \\
\text { Nanotechnology }\end{array}$ & $\begin{array}{l}\text { The monitoring system for R\&D projects in the context of the } \\
C C N \text { is the one which is used for all R\&D grants in Germany. It } \\
\text { is well known to the researchers (from university and industry), } \\
\text { not too exhaustive but fulfills the requirements to assure } \\
\text { accountability. }\end{array}$ & $\begin{array}{l}\text { Almost no MAP specific criteria and procedures except from } \\
\text { the monitoring evaluation }\end{array}$ \\
\hline UK & Technopolis & $\begin{array}{l}\text { Partners in } \\
\text { Innovation, PII }\end{array}$ & Project-specific, fast turnaround, good data capture & $\begin{array}{l}\text { As with any monitoring system, it is very poor at estimating } \\
\text { future impacts. }\end{array}$ \\
\hline ES & $\begin{array}{l}\text { Asociación } \\
\text { Unitec }\end{array}$ & $\begin{array}{l}\text { PCTI } \\
\text { Cooperacion }\end{array}$ & $\begin{array}{l}\text { Close relationship between the Scientific Officer and the } \\
\text { consortium which allows for overcoming recurrent } \\
\text { administrative /bureaucratic obstacles. }\end{array}$ & $\begin{array}{l}\text { The electronic tool used is not too user friendly, but allows for } \\
\text { efficient monitoring and reporting. }\end{array}$ \\
\hline SL & PMIS & $\begin{array}{l}\text { Toolmaker } \\
\text { Network }\end{array}$ & $\begin{array}{l}\text { Amount of data and information: fine } \\
\text { Collection and controlling effort: fine } \\
\text { Basis for funding decision: fair }\end{array}$ & NA \\
\hline IE & $\begin{array}{l}\text { SEAI (formerly } \\
\text { IEC) }\end{array}$ & Energy RDD & $\begin{array}{l}\text { Good control of costs, and reasonable monitoring of technical } \\
\text { results/impacts }\end{array}$ & Too soon to say, but possibly longer term evaluation of results \\
\hline BE & IIWT & VIS & $\begin{array}{l}\text { Daily management of the projects are subjected to the } \\
\text { programme managers Follow-up is easy }\end{array}$ & $\begin{array}{l}\text { Administrative burden must be kept simple for both sides } \\
\text { (clients and IWT) }\end{array}$ \\
\hline
\end{tabular}




\begin{tabular}{|c|c|c|c|c|}
\hline Country & Organisation & MAP & 2. Project Evaluation & 2.1. When is it carried out? \\
\hline & & & & \\
\hline AT & TIG & K plus & $\begin{array}{l}\text { There are ex-ante and interim project evaluations, ex-post } \\
\text { evaluations will be carried out (undecided yet whether on } \\
\text { project or programme level); } 43 \text { ex-ante evaluations, } 8 \text { interim } \\
\text { evaluations to date }\end{array}$ & Interim evaluation: First quarter of 4 th year \\
\hline SE & VINNOVA & $\begin{array}{l}\text { Competence } \\
\text { Centres } \\
\text { Programme }\end{array}$ & $\begin{array}{l}\text { Centre }(=\text { project }) \text { evaluations, especially the interim } \\
\text { evaluations are a key part of the programme. }\end{array}$ & $\begin{array}{l}\text { Ex ante: In 1993-94 when the MAP was launched (only one } \\
\text { call so far). Interim: At the end of each stage (1997, 2000, } \\
\text { 2003); Ex post: Not yet decided. }\end{array}$ \\
\hline $\mathrm{HU}$ & MoE & KKK & $\begin{array}{l}\text { The followings are valid only for the KKK program: ex-ante } \\
\text { evaluation: } 1 \text { month after the deadline for the call for proposal; } \\
\text { interim avalutaion: at project milestones defined by the } \\
\text { applicants; ex-post evaluation: after the final reports. The } \\
\text { evaluators: ex-ante: external experts, mainly peer-review; } \\
\text { interim: program managers plus external experts and siet } \\
\text { visits; ex-post: Agency and external experts. }\end{array}$ & $\begin{array}{l}\text { The followings are valid only for the KKK program: ex-ante } \\
\text { evaluation: } 1 \text { month after the deadline for the call for proposal; } \\
\text { interim avalutaion: at project milestones defined by the } \\
\text { applicants; ex-post evaluation: after the final reports. }\end{array}$ \\
\hline EE & MoEA & $\begin{array}{l}\text { CC programme } \\
\text { design }\end{array}$ & $\begin{array}{l}\text { Everything described here is a plan, because we have no } \\
\text { established any CCs yet }\end{array}$ & $\begin{array}{l}\text { Interim evaluation in the beginning of the } 4 \text { th year of the CC. } \\
\text { Ex-post after the } 7 \text { th year. }\end{array}$ \\
\hline & & & & \\
\hline $\mathrm{DE}$ & $\mid \mathrm{ISI}$ & $\begin{array}{l}\text { CC } \\
\text { Nanotechnology }\end{array}$ & $\begin{array}{l}\text { Interim evaluations at project level take place every half year, } \\
\text { based on the interim reports, and are carried out by the project } \\
\text { agencies: the result is a "stop-or-go“ decision. The ex post } \\
\text { evaluation of R\&D projects is carried out } 4 \text { months after } \\
\text { termination of the legal year or } 6 \text { weeks after termination of the } \\
\text { project, also by the project agencies. Possible consequences } \\
\text { are - in the case that the project was a complete failure - a } \\
\text { refusal of the final payment. }\end{array}$ & see 2 \\
\hline UK & Technopolis & \begin{tabular}{|l} 
Partners in \\
Innovation, PII
\end{tabular} & $\begin{array}{l}\text { ex ante appraisal is run annually, at two points in time (Oct, } \\
\text { March). Interim evaluations occur only with the larger projects, } \\
\text { and timing is project specific. Ex-post project evaluation is } \\
\text { carried out for every project, by the lead partner, as part of } \\
\text { their final claim, and has to be approved by the project officer, } \\
\text { who in turn will rate the work. }\end{array}$ & Project specific \\
\hline ES & $\begin{array}{l}\text { Asociación } \\
\text { Unitec }\end{array}$ & $\begin{array}{l}\text { PCTI } \\
\text { Cooperacion }\end{array}$ & Monitoring and evaluations are done jointly & Ex-ante, interim \& ex-post project evaluation are done yearly. \\
\hline SL & PMIS & $\begin{array}{l}\text { Toolmaker } \\
\text { Network }\end{array}$ & ex-ante, ex-post & 4 months \\
\hline IE & $\begin{array}{l}\text { SEAI (formerly } \\
\text { IEC) }\end{array}$ & Energy RDD & $\begin{array}{l}\text { Longer projects would have ongoing monitoring though not } \\
\text { evaluation. Ex post evaluation carried out as part of closing the } \\
\text { contract and assessing final deliverables. Longer term } \\
\text { evaluation: may depend on resources available - too soon to } \\
\text { comment at this stage. }\end{array}$ & See previous response \\
\hline BE & IIWT & VIS & $\begin{array}{l}\text { In the selection procedure of the projects there is an ex ante } \\
\text { evaluation. After } 24 \text { months there is mid-term monitoring (not a } \\
\text { real evaluation). At hte end there is planned to be an in depth } \\
\text { evaluation (first projects of the VIS will end in 2005) }\end{array}$ & $\begin{array}{l}\text { Mid-term monitoring : in the period month } 20 \text { to } 24 \text {, End } \\
\text { evalaution in the period month } 44 \text { to } 48\end{array}$ \\
\hline
\end{tabular}




\begin{tabular}{|c|c|c|c|c|}
\hline & & & 2. Project Evaluation & \\
\hline Country & Organisation & MAP & 2.2. What kind of info do you use for the project evaluation & $\begin{array}{l}\text { 2.3. How do you take specific MAP characteristics into } \\
\text { account? }\end{array}$ \\
\hline AT & TIG & K plus & $\begin{array}{l}\text { Main input for mid-term evaluation is the "Core Document" } \\
\text { containing a report for the years } 1-3 \text { and the plan for the years } \\
\text { 4-7; This is supplemented by the TIG report and a short } \\
\text { statement by the ERP-Fonds (Relation to industry, technology } \\
\text { transfer, regional aspects, strategic position); Central for the } \\
\text { mid-term evaluation is a } 1,5 \text { day discussion with the centre and } \\
\text { its partners (site visit); Site visit and Final Report is structured } \\
\text { by a questionnaire that builds on the indicators for the } \\
\text { evaluation }\end{array}$ & $\begin{array}{l}\text { Three members of the evaluation team visit all the centres } \\
\text { (“Standing Group") enabling learning processes and } \\
\text { "organisational memory"; The result of the evaluation (final } \\
\text { report) is fed back to the centres and serve as an input into the } \\
\text { agreement and funding contract for the years 5-7 }\end{array}$ \\
\hline SE & VINNOVA & \begin{tabular}{|l|} 
Competence \\
Centres \\
Programme
\end{tabular} & $\begin{array}{l}\text { Special centre reports according to MAP-specific VINNOVA } \\
\text { guidelines. }\end{array}$ & $\begin{array}{l}\text { The first interim evaluation in } 1997 \text { (after } 1 \frac{1}{2} \text { years) focused } \\
\text { on MAP-specific "added centre values". } \\
\text { In the mid-term evaluation (after } 41 / 2 \text { years) "Development as } \\
\text { a Competence Centre. Added values" was one of three criteria. }\end{array}$ \\
\hline $\mathrm{HU}$ & MoE & KKK & $\begin{array}{l}\text { scientific value and usefulness of teh results, centre } \\
\text { management methods, financial data, number of PhD students, } \\
\text { number of normal students and number of reserchers } \\
\text { (aacademic and industrial) involved in the projects. }\end{array}$ & $\begin{array}{l}\text { In the case of other R\&D projects the site visits are based on } \\
\text { random selections, about } 10 \% \text { of the projects are evaluated } \\
\text { this way. In case of MAP projects the site visits are obligatory. } \\
\text { Besides, the program manager and a responsible person from } \\
\text { the National Office for Research and Technology takes part on } \\
\text { the Board meetings of the centres, and also on their Scientific } \\
\text { meetings or special conferences. On these occasions we } \\
\text { inform the centres about their overall performance, or better to } \\
\text { say what we think where they are in compariosn to other } \\
\text { centres. It is a kind of benchmarking, and works well. }\end{array}$ \\
\hline $\mathrm{EE}$ & MoEA & $\begin{array}{l}\text { CC programme } \\
\text { design }\end{array}$ & $\begin{array}{l}\text { For interim and ex-post evaluation the CCs will deliver a report } \\
\text { about their activities, the comparison of planned and achieved } \\
\text { results and the fiscal information. Also the biannual monitoring } \\
\text { reports will be used. }\end{array}$ & \\
\hline $\mathrm{DE}$ & $|S|$ & $\begin{array}{l}\text { CC } \\
\text { Nanotechnology }\end{array}$ & see 1.3 & $\begin{array}{l}\text { The project evaluation system in the context of the CCN is the } \\
\text { one which is used for all R\&D grants in Germany. It is well } \\
\text { known to the researchers (from university and industry), not } \\
\text { too exhaustive but fulfills the requirements to assure } \\
\text { accountability. Particularities of MAPs are not taken into } \\
\text { account }\end{array}$ \\
\hline UK & Technopolis & $\begin{array}{l}\text { Partners in } \\
\text { Innovation, PII }\end{array}$ & $\begin{array}{l}\text { Interim evalutions consider feasibility in light of progress, } \\
\text { quality of work performed, satsifaction of consortium members, } \\
\text { record of outputs, revised estimate of likely impact, a decision } \\
\text { to continue or not. Ex post evaluations will mainly comprise a } \\
\text { reference to reports and other outputs, a description of } \\
\text { anticipated impact, with quantitative estimates if possible, and } \\
\text { a rating of project performance (quality of work, } \\
\text { responsiveness, impact) by the project officer. }\end{array}$ & $\begin{array}{l}\text { ex ante and interim evaluations will consider } \\
\text { support/satisfaction of consortium members }\end{array}$ \\
\hline ES & $\begin{array}{l}\text { Asociación } \\
\text { Unitec }\end{array}$ & \begin{tabular}{|l} 
PCTI \\
Cooperacion
\end{tabular} & $\begin{array}{l}\text { Monitoring data. Interviews / site visits, where correspondence } \\
\text { between WP and results is checked. }\end{array}$ & MAP characteristics are not normally taken into account. \\
\hline $\mathbf{S L}$ & PMIS & $\begin{array}{l}\text { Toolmaker } \\
\text { Network }\end{array}$ & monitoring data, interviews, site visits, public presentations & $\begin{array}{l}\text { integration of specific learning elements and feedback loops, } \\
\text { use of MAP specific methods }\end{array}$ \\
\hline $\mathrm{IE}$ & $\begin{array}{l}\text { SEAI (formerly } \\
\text { IEC) }\end{array}$ & Energy RDD & Ex post & $\begin{array}{l}\text { Hasn't been decided yet; specific MAP characteristics (multi- } \\
\text { actor, etc) should be taken into account but may not be an } \\
\text { explicit or central element of evaluation }\end{array}$ \\
\hline BE & IWT & VIS & $\begin{array}{l}\text { interim : written documents and reports from the project } \\
\text { coordinator, reports of the meeting of the client groups } \\
\text { accompanying the projects; ex post : we hope that we also do } \\
\text { a customer satisfaction study }\end{array}$ & $\begin{array}{l}\text { The key indicators that the project coordinators have to sent to } \\
\text { us every three months take into a account of the Map- } \\
\text { characteristics) and also the networking issues between } \\
\text { projects (eg signposting to each other services and common } \\
\text { projects) }\end{array}$ \\
\hline
\end{tabular}




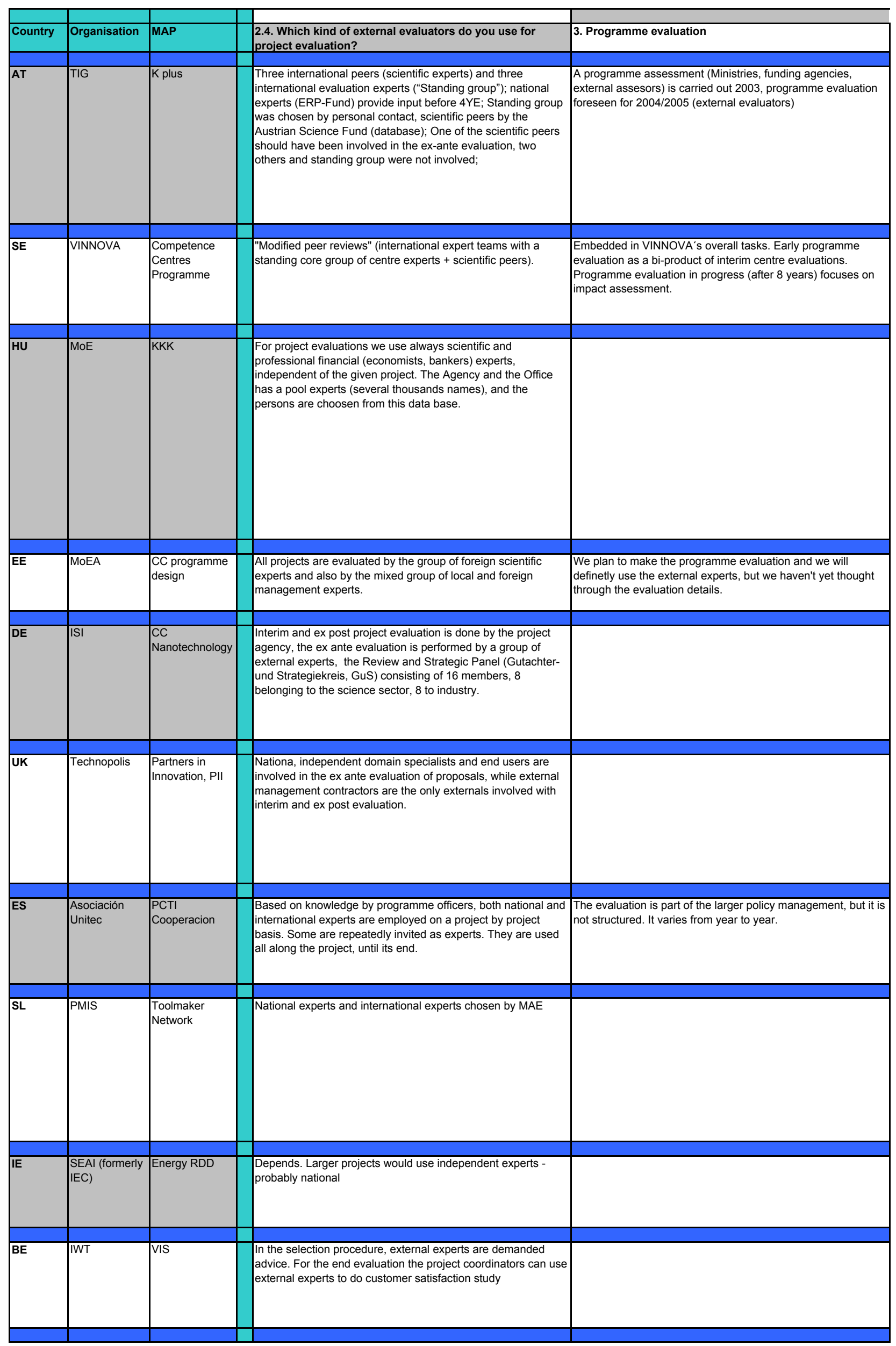




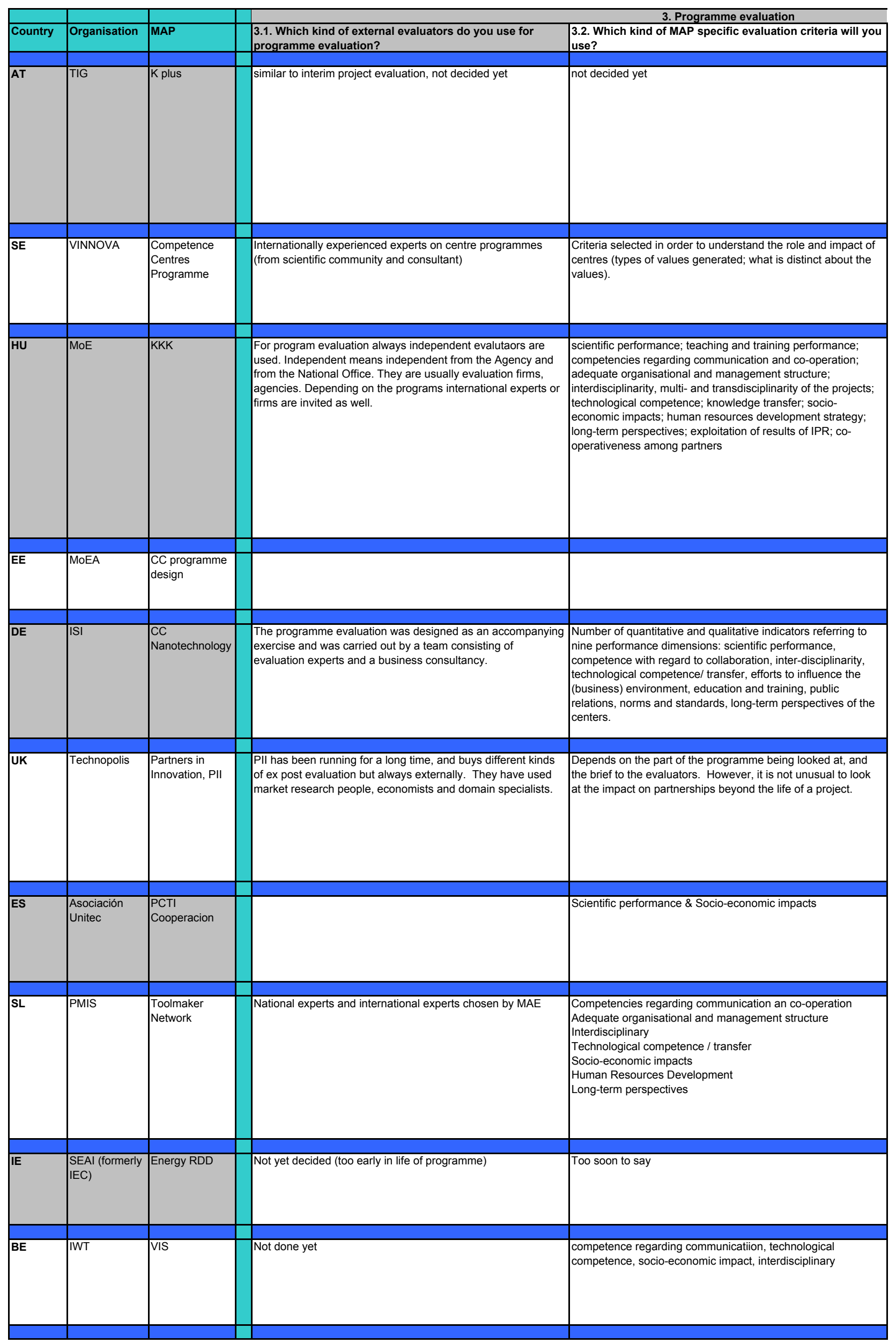




\begin{tabular}{|c|c|c|c|c|}
\hline Country & Organisation & MAP & 3.3. Which kind of methods will you use? & $\begin{array}{l}\text { 3.4. What is the connection between project evaluation and } \\
\text { programme evaluation? }\end{array}$ \\
\hline AT & TIG & K plus & not decided yet & $\begin{array}{l}\text { interim project evaluation serves as input for programme } \\
\text { evaluation }\end{array}$ \\
\hline SE & VINNOVA & $\begin{array}{l}\text { Competence } \\
\text { Centres } \\
\text { Programme }\end{array}$ & $\begin{array}{l}\text { A combination of traditional (case studies, success stories, } \\
\text { mapping) and partly new methods (i e Knowledge Value } \\
\text { Collectives). }\end{array}$ & $\begin{array}{l}\text { From the programme objectives five strict criteria for the ex- } \\
\text { ante selection of centres were worked out. Ten performance } \\
\text { criteria formed a basis for the three interim evaluations of the } \\
\text { centres. The 3rd of them gives an input to the impact } \\
\text { evaluation of the programme. }\end{array}$ \\
\hline $\mathrm{HU}$ & MoE & KKK & $\begin{array}{l}\text { Program evalutaion: innovation studies; econometric models; } \\
\text { bibliometric and patent analysis; control group approaches; } \\
\text { cost/benefit analysis; expert panel and peer review; field and } \\
\text { case studies; technology assessment, foresight studies; } \\
\text { benchmarking. There is a Strategy Handbook on Evaluations } \\
\text { put down by former OMFB (National Committe for } \\
\text { Technological Development: now it is the Agency (KPI) and the } \\
\text { National Office for Reasearch and Technology) }\end{array}$ & $\begin{array}{l}\text { The different parts of the results of the project evaluations are } \\
\text { part of the program evaluation. For the program evaluations } \\
\text { also survays are carried out: for most of the R\&D programmes } \\
\text { the survays are based on saplnig, but the case of some } \\
\text { specific porgrammes (high-tech and KKK) all the partners are } \\
\text { give a questionnaire. }\end{array}$ \\
\hline $\mathrm{EE}$ & MoEA & $\begin{array}{l}\text { CC programme } \\
\text { design }\end{array}$ & & \\
\hline $\mathrm{DE}$ & $\mid \mathrm{SI}$ & $\begin{array}{l}\text { CC } \\
\text { Nanotechnology }\end{array}$ & $\begin{array}{l}\text { Analysis of (Project and Center) Documents Qualitative } \\
\text { Interviews, Mail Survey, Network Analysis, Workshops, } \\
\text { Training }\end{array}$ & $\begin{array}{l}\text { There is no link between the (internal) monitoring of projects } \\
\text { and the (external) evaluation, for two reasons: (1) particularity } \\
\text { of the German promotional scheme and (2) fear that internal } \\
\text { project details are disseminated to a wider public (formulated } \\
\text { by the project agencies). }\end{array}$ \\
\hline UK & Technopolis & $\begin{array}{l}\text { Partners in } \\
\text { Innovation, PII }\end{array}$ & $\begin{array}{l}\text { Peer review of quality, market research to gauge participant } \\
\text { satisfaction, case studies of project outcomes and impacts }\end{array}$ & $\begin{array}{l}\text { end-of-project assessments are aggregated in a reporting } \\
\text { system, and in some cases programme evaluators have used } \\
\text { these data to perform statistical analyses associating impacts } \\
\text { with other features and to guide investigations towards } \\
\text { samples of high and low performing projects }\end{array}$ \\
\hline ES & $\begin{array}{l}\text { Asociación } \\
\text { Unitec }\end{array}$ & \begin{tabular}{|l|} 
PCTI \\
Cooperacion
\end{tabular} & Benchmarking, econometric models, innovation studies. & $\begin{array}{l}\text { Project derived data serve to calculate programme indicators. } \\
\text { Programme evaluation is based on "numeric, cold" data from } \\
\text { projects, and done asynchronously, when requested by } \\
\text { manager }\end{array}$ \\
\hline SL & PMIS & $\begin{array}{l}\text { Toolmaker } \\
\text { Network }\end{array}$ & $\begin{array}{l}\text { innovations studies } \\
\text { econometric models } \\
\text { bibliometric and patent analysis } \\
\text { cost benefit analysis } \\
\text { expert panel / peer review } \\
\text { field / case studies } \\
\text { network analysis } \\
\text { foresight / technology assessment } \\
\text { benchmarking }\end{array}$ & a strong connection between project and programme level \\
\hline IE & $\begin{array}{l}\text { SEAl (formerly } \\
\text { IEC) }\end{array}$ & Energy RDD & Too soon to say & Too soon to say \\
\hline BE & IWT & VIS & not decided yet & $\begin{array}{l}\text { the programme evaluation is not the same as the sum op the } \\
\text { project evaluations. Programme evaluations goes about the } \\
\text { effect of all the projects together and is a more comprehensive } \\
\text { exercice. }\end{array}$ \\
\hline
\end{tabular}




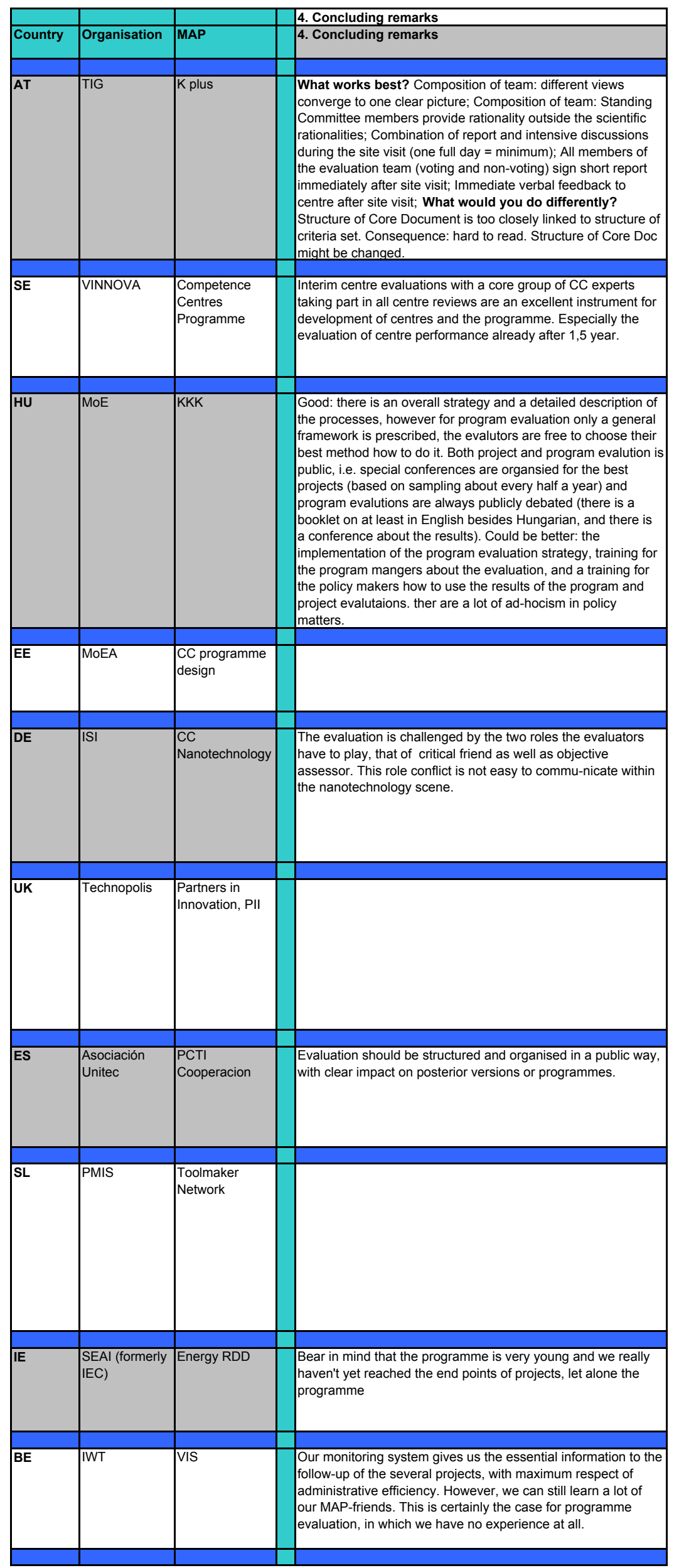


12.3.7 StarMAPs

\begin{tabular}{|c|c|c|c|c|}
\hline & & 1. Governance & 2. Design & \\
\hline Country & MAP & 1.1 Governance & $\begin{array}{l}\text { 2.1. Introduction/overview on } \\
\text { design of this specific MAP }\end{array}$ & $\begin{array}{l}\text { 2.2. MAP guidelines, underlying } \\
\text { documents }\end{array}$ \\
\hline AU & CRC & $\begin{array}{l}\text { Minister of Education Science and } \\
\text { Training } \\
\text { CRC Committee } \\
\text { CRC Secretariat (--> Administration) } \\
\text { Expert Panel (Life Science \& Physical } \\
\text { Science and Engineering) } \\
\text { Visitors }\end{array}$ & $\begin{array}{l}\text { design process started in } 1989 \text { (chief } \\
\text { scientist - prime minister). Got topic of } \\
\text { elections and was approved in } 1990 .\end{array}$ & $\begin{array}{l}\text { Guidleines have undergone several } \\
\text { modifications (e.g. stornger emphasis } \\
\text { on SMEs) but principly repained } \\
\text { unchanged }\end{array}$ \\
\hline CA & NCE & $\begin{array}{l}\text { Ministry: member of Steering } \\
\text { Committee; StCom (= Deputy Minister } \\
\text { + } 3 \text { Presidents of Granting Councils); } \\
\text { Agency = NCE directorate: } \\
\text { organisation of all aspects of } \\
\text { implementation; External experts: } \\
\text { high level for selection committeed } \\
\text { (recommendations) + peers for } \\
\text { Interdisciplinary expert panel } \\
\text { (technical reports) }\end{array}$ & $\begin{array}{l}\text { no linear process; Minister } \\
\text { announced in } 1989 \text { programme } \\
\text { during elections }+10 \text { evaluation } \\
\text { criteria; original intention: centre } \\
\text { programme; pr. Designed in } 1 \text { year } \\
\text { jointly with Research Councils }\end{array}$ & $\begin{array}{l}\text { Pr. Follow laws from Tresury Board - } \\
\text { evaluation every } 5 \text { year required }\end{array}$ \\
\hline FI & Technology Programmes & $\begin{array}{l}\text { Each programme has a steering } \\
\text { group, an external programme } \\
\text { director and a responsible person at } \\
\text { Tekes. Large programmes } \\
\text { additionally are divided into theme } \\
\text { areas with its own steering group. } \\
\text { projects might have their own } \\
\text { advisory board or steering group as } \\
\text { well. }\end{array}$ & \begin{tabular}{|l|} 
result of the recommendations of \\
several committees appointed by the \\
Council of State in the early 1980 to \\
overcome the economic recession \\
and the problems based on structural \\
changes
\end{tabular} & $\begin{array}{l}\text { There is a manual of about } 15 \text { pages } \\
\text { for tasks of the programme manager } \\
\text { and the steering group. Additionally, } \\
\text { there exist a general guideline for } \\
\text { evaluations of technology } \\
\text { programmes }\end{array}$ \\
\hline FR & PREDIT & $\begin{array}{l}\text { designed and funded by } 4 \text { ministries } \\
\text { and } 2 \text { national agencies; managed by } \\
11 \text { operational groups }\end{array}$ & $\begin{array}{l}\text { 3rd PREDIT programme launched in } \\
\text { March } 2002\end{array}$ & $\begin{array}{l}\text { small number of basis documents } \\
\text { which provide the legal basis }\end{array}$ \\
\hline NL & TS & $\begin{array}{l}\text { Ministry of Economic Affairs } \\
\text { responsible for programme design, } \\
\text { budgeting and supervision; Senter = } \\
\text { programme manager; external } \\
\text { experts for project evaluations and ex- } \\
\text { post evaluations }\end{array}$ & $\begin{array}{l}\text { to reinforce the co-operation between } \\
\text { firms and knowledge institutes, } \\
\text { government announced widening of } \\
\text { its policy for promoting strategic co- } \\
\text { operation between high-tech firms } \\
\text { and the public research infrastructure } \\
\text { by a new technological co-operation } \\
\text { scheme, BTS }\end{array}$ & some legal acts \\
\hline NO & TEFT & $\begin{array}{l}\text { TEFT is a national sub-programme } \\
\text { within the MOBI programme and is } \\
\text { financed by the Ministry for Local } \\
\text { Government and Regional Affairs and } \\
\text { the Ministry of Trade and Industry } \\
\text { through the RCN. TEFT is governed } \\
\text { by a combined mechanism of a } \\
\text { board, or programme committee, } \\
\text { elected by the RCN, a programme } \\
\text { manager at the RCN, an } \\
\text { implementation manager at SINTEF } \\
\text { and a TEFT steering group. }\end{array}$ & \begin{tabular}{|l|} 
In 1989, the TEFT forerunner \\
programme DTS was launched and \\
ran for five years. basic idea was \\
perceived need of firms to enhance \\
their technological capacity through \\
technology assistance (target group = \\
SMEs); programme evaluated and \\
further devolopped - TEFT
\end{tabular} & $\begin{array}{l}\text { National Budget; Programme } \\
\text { catalogue for } 2000 \text { from the Research } \\
\text { Council of Norway; }\end{array}$ \\
\hline
\end{tabular}




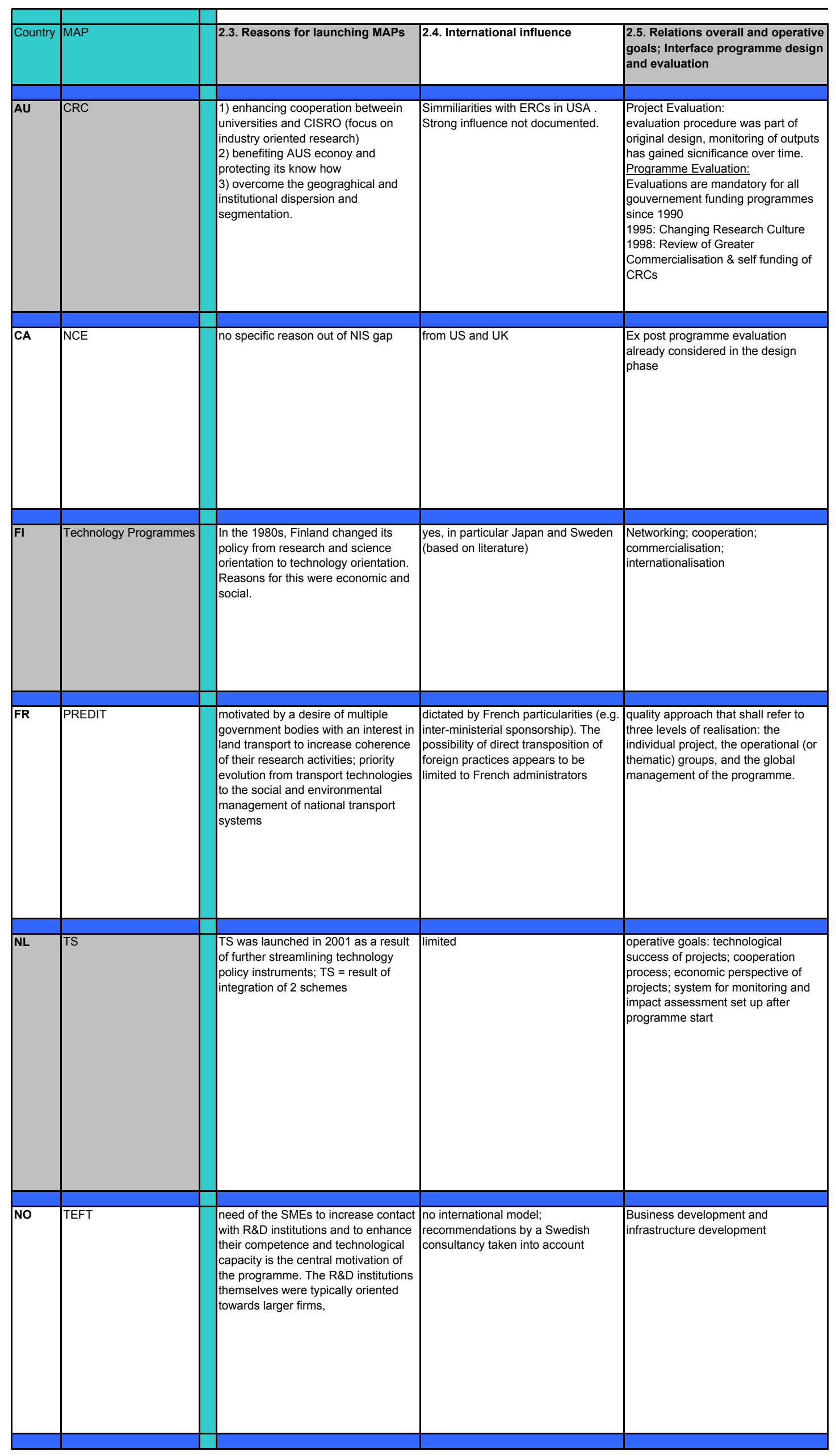




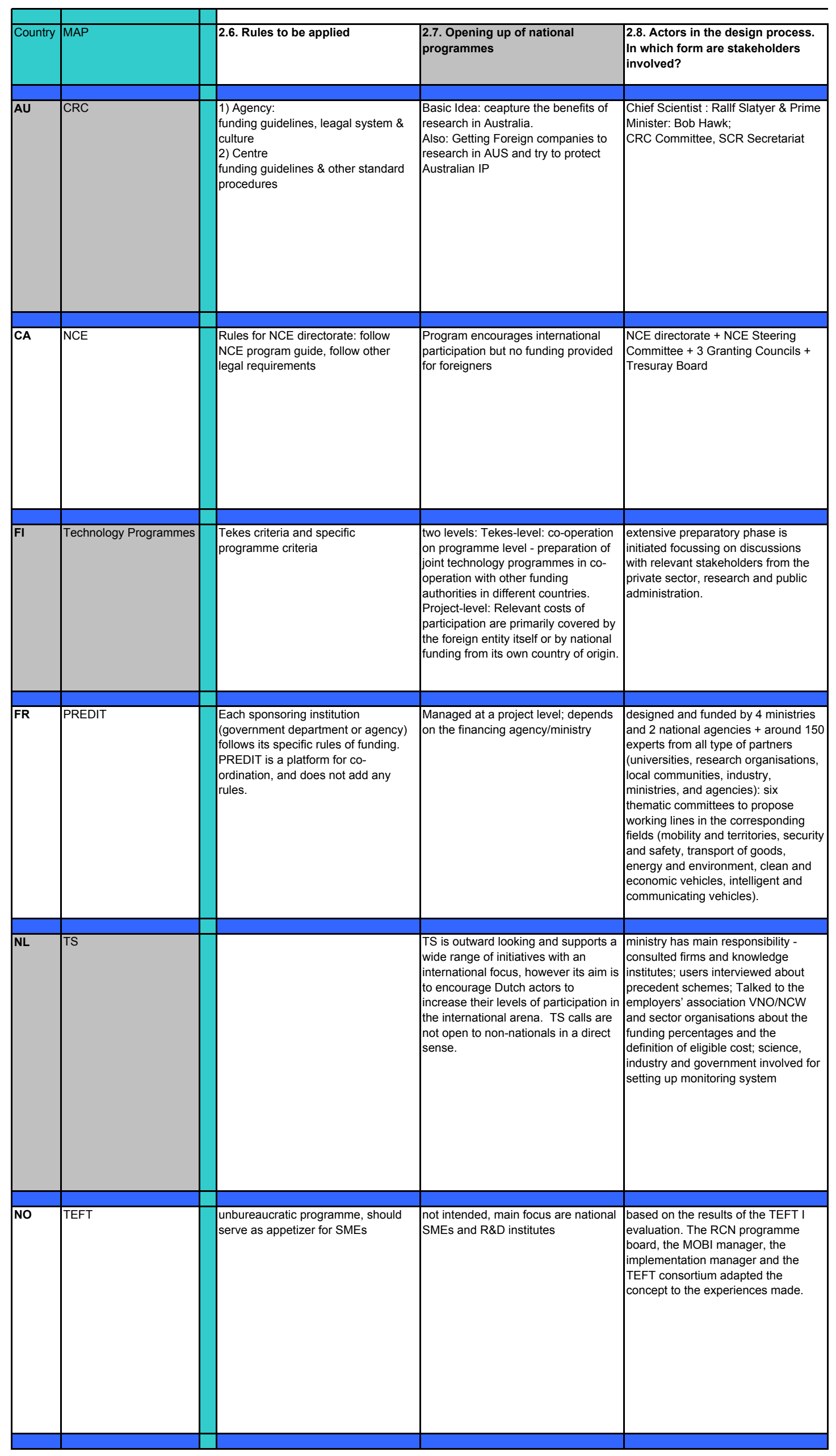




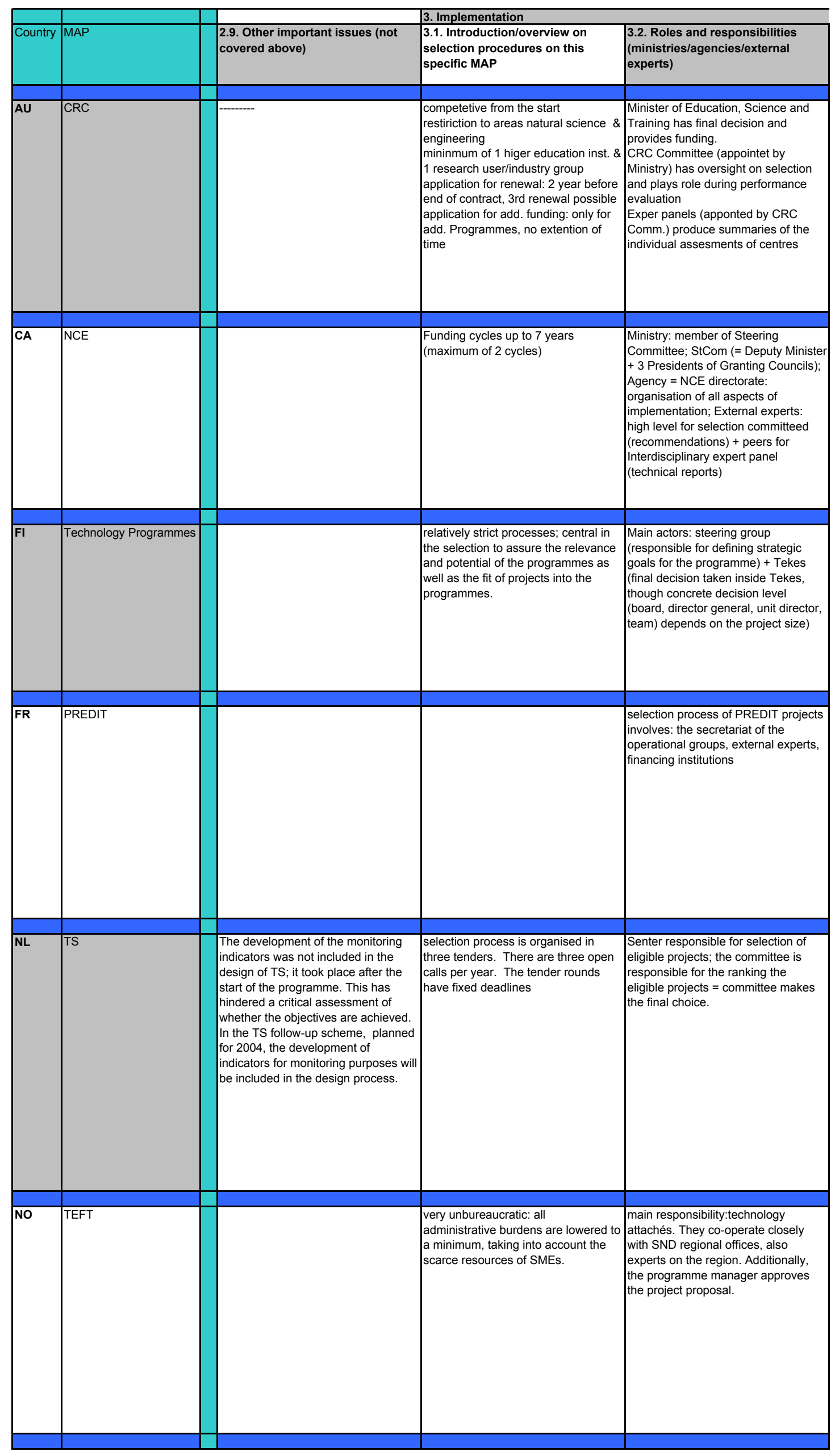




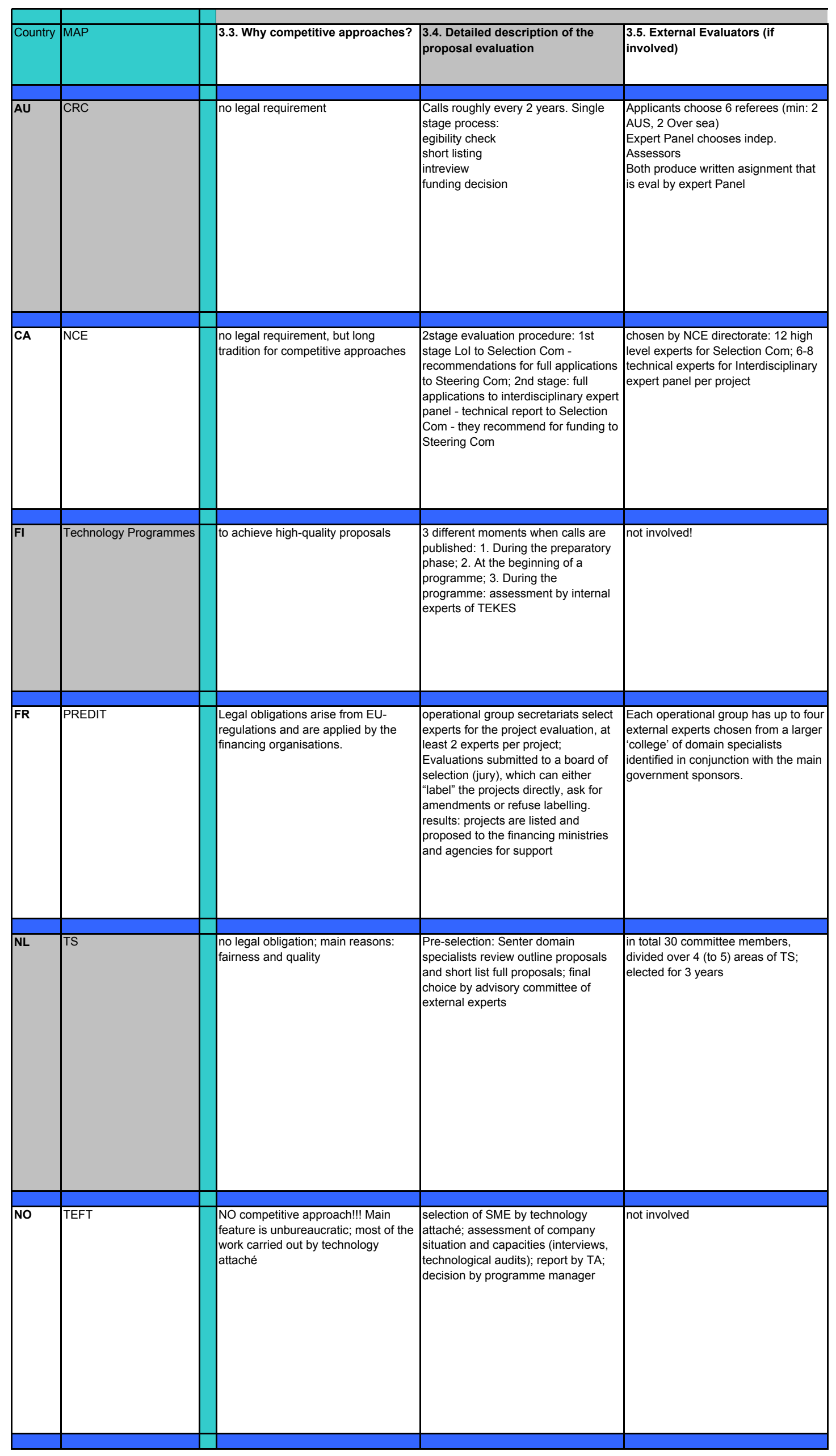




\begin{tabular}{|c|c|c|c|c|}
\hline & & & & 4. Contract Negotiations, funding pr \\
\hline Country & MAP & 3.6. Evaluation criteria & $\begin{array}{l}\text { 3.7. Other important issues (not } \\
\text { mentioned above) }\end{array}$ & 4.1. Introduction \\
\hline AU & CRC & $\begin{array}{l}\text { OUTCOME: } \\
\text { Objectives of the CRS } \\
\text { Qulaity and Relevance } \\
\text { OUTPUT: } \\
\text { Stratigy for utilisation \& } \\
\text { Commercialisation } \\
\text { Education \& Training } \\
\text { Collaborative arrangements } \\
\text { INPUT \& intermediate OUTPUT: } \\
\text { Resources and Budget } \\
\text { Management Structure } \\
\text { Performance Evaluation }\end{array}$ & $\begin{array}{l}\text { State gov. Coach applicants or } \\
\text { provide support }\end{array}$ & $\begin{array}{l}\text { Carried out by CRC Secret. } \\
\text { Time: } 4 \text { months. } \\
\text { Participants develop Centre } \\
\text { Agreement } \\
\text { CRC Secritariat \& Key personal } \\
\text { develop Commonwealth Agreement } \\
\text { Model for Centre Agreement is } \\
\text { provided. } \\
\text { Commonwealth Agreement is highly } \\
\text { standardised }\end{array}$ \\
\hline CA & NCE & $\begin{array}{l}\text { 1. Excellence of Research program; } \\
\text { 2. Development of Highly Qualified } \\
\text { Personnel; } 3 . \text { Networking and } \\
\text { Partnership; } 4 . \text { Knowledge and } \\
\text { Technology Exchange and } \\
\text { Exploitation; } 5 . \text { Management of } \\
\text { Network }\end{array}$ & & $\begin{array}{l}\text { part carried out between NCE } \\
\text { directorate and NCEs; main part is } \\
\text { network agreement between all } \\
\text { participants in an NCE }\end{array}$ \\
\hline FI & Technology Programmes & $\begin{array}{l}\text { Programme criteria: networking with } \\
\text { other companies; joint ventures; the } \\
\text { use of local SME subcontractors in } \\
\text { the case of larger companies; } \\
\text { participation in national technology } \\
\text { programs; contracting of services } \\
\text { from Finnish research institutes; } \\
\text { universities and promotion of } \\
\text { international co-operation }\end{array}$ & $\begin{array}{l}\text { Selection of new technology } \\
\text { programmes: idea for a new } \\
\text { technology programme comes either } \\
\text { from Tekes, a big company or } \\
\text { business association or as a follow up } \\
\text { from another programme }\end{array}$ & \begin{tabular}{|l|} 
applying institute receives short \\
report and information on the funding \\
decision and the contract. \\
negotiations are a continuous \\
process with the project manager. \\
timespan between the turning in of \\
the proposal until the contract is \\
signed is about 40 days.
\end{tabular} \\
\hline FR & PREDIT & $\begin{array}{l}\text { Appropriateness in relation to the } \\
\text { objectives of the operational group; } \\
\text { Originality (potential contribution to } \\
\text { the state of the art); Relevance of the } \\
\text { methodology (scientific quality); Cost } \\
\text { appreciation (means asked for); } \\
\text { Credibility of the team; Valorisation of } \\
\text { results }\end{array}$ & & \begin{tabular}{|l|} 
PREDIT supports in different ways \\
(subsidies, refundable loans) \\
research contracts, financing of \\
doctoral theses, subsidies for \\
employment of researchers in \\
industry: final selection and \\
negotiation of contracts, operated by \\
the individual financing institutions \\
(the four ministries and two agencies) \\
according to their particular rules and \\
procedures.
\end{tabular} \\
\hline$\overline{N L}$ & TS & $\begin{array}{l}\text { several eligibility criteria (not listed } \\
\text { here); for ranking important: } \\
\text { cooperation, technological innovation, } \\
\text { economic perspective }\end{array}$ & $\begin{array}{l}\text { support for applicants: 1) Senter asks } \\
\text { potential applicants to send them a } \\
\text { one page description; 2) Application } \\
\text { manual for applicants }\end{array}$ & $\begin{array}{l}\text { average negotiating time is a few } \\
\text { weeks; Senter tries to visit } \\
\text { organisations at the start of a project } \\
\text { to make sure that conditions, etc. are } \\
\text { clear. }\end{array}$ \\
\hline NO & TEFT & $\begin{array}{l}\text { TA looks at following issues at SMEs: } \\
\text { the general manager's ambitions, } \\
\text { motivation, and competence; strategic } \\
\text { capabilities; financial situation (the } \\
\text { firm should possess resources } \\
\text { suffcient to embark on relevant } \\
\text { activities); willingness to deploy } \\
\text { financial resources; ownership; R\&D } \\
\text { competence and experience; R\&D } \\
\text { intensity; knowledge level and } \\
\text { competence }\end{array}$ & $\begin{array}{l}\text { The technology attachés come from } \\
\text { four main research institutes of } \\
\text { Norway: SINTEF (the Foundation for } \\
\text { Scientific and Industrial Research at } \\
\text { the Norwegian Institute of } \\
\text { Technology), IFE (Institute for Energy } \\
\text { Technology), RF (Rogaland } \\
\text { Research: Research activities within } \\
\text { Petroleum, Aquatic Environment, } \\
\text { Social Science and Business } \\
\text { Development) and FFI (Norwegian } \\
\text { Defence Research Establishment). } \\
\text { They are senior advisors, who have } \\
\text { long experience with/in industrial } \\
\text { SME's and/or research institutes. }\end{array}$ & $\begin{array}{l}\text { The results of the interview and } \\
\text { technology audit are the basis for the } \\
\text { funding decision and become } \\
\text { resumed by the attaché in a pilot } \\
\text { project report. This together with the } \\
\text { project proposal including the budget } \\
\text { is presented to the programme } \\
\text { committee and the programme } \\
\text { manager at SINTEF who take the } \\
\text { final decision. }\end{array}$ \\
\hline
\end{tabular}




\begin{tabular}{|c|c|c|c|c|}
\hline & & \multicolumn{3}{|l|}{ inciples and consortia } \\
\hline Country & MAP & 4.2. Funding & 4.3. Consortia & 4.4. IPR \\
\hline$A U$ & CRC & $\begin{array}{l}\text { Eligible Costs: } \\
\text { Salaries (researcher, professionsl } \\
\text { staff) } \\
\text { Capital items over } \$ 20000 \text { (incl. } \\
\text { Equipment) } \\
\text { Administraion Overheads, other } \\
\text { salaries, salary on-costs,... }\end{array}$ & $\begin{array}{l}\text { apart fom the rquest for } 1 \text { higher } \\
\text { education institute there are no legal } \\
\text { requirements for the consorti. }\end{array}$ & $\begin{array}{l}\text { results are owned by the CRC and } \\
\text { given (terms negotiated) to parners. }\end{array}$ \\
\hline CA & NCE & $\begin{array}{l}\text { for a maximum of } 14 \text { years (after that } \\
\text { "sunset"); Average NCE funding per } \\
\text { year between } 1,9 \text { and } 3,7 \text { Mio } € \text { from } \\
\text { NCE programme - more than doubled } \\
\text { by private sector }\end{array}$ & $\begin{array}{l}\text { no specific requirement on the form of } \\
\text { consortia; practice is: half uni and half } \\
\text { private }\end{array}$ & $\begin{array}{l}\text { Standard NCE Network Agreement: } \\
\text { Ownership of Network-Supported } \\
\text { Intellectual Property (NSIP) shall be } \\
\text { determined by applicable Canadian } \\
\text { law and the policies of the relevant } \\
\text { participating institutions. }\end{array}$ \\
\hline FI & Technology Programmes & $\begin{array}{l}\text { Usually about half of the costs of the } \\
\text { programmes are financed by Tekes. } \\
\text { The second half comes from } \\
\text { participating companies. In several } \\
\text { programmes there are co-financiers } \\
\text { like the Academy of Finland and } \\
\text { ministries }\end{array}$ & $\begin{array}{l}\text { Basically all national companies and } \\
\text { research can apply for funding, } \\
\text { though the funding is especially } \\
\text { oriented towards SMEs: If an } \\
\text { international institute and company } \\
\text { wishes to participate in a technology } \\
\text { programme, it must receive funding } \\
\text { from its country of origin }\end{array}$ & $\begin{array}{l}\text { widely standardised for all } \\
\text { programmes. research results } \\
\text { become public after the completion of } \\
\text { a programme. to have access to the } \\
\text { results already beforehand, } \\
\text { companies buy themselves into the } \\
\text { steering groups, so they can } \\
\text { participate in the results } \\
\text { dissemination activities }\end{array}$ \\
\hline FR & PREDIT & $\begin{array}{l}\text { PREDIT } 3 \text { has an overall budget of } \\
\text { EUR } 306 \text { million (public funds) for the } \\
\text { period } 2002-2006, \text { covered as follows } \\
\text { by six funding institutions ( } 4 \\
\text { ministries and } 2 \text { agencies) }\end{array}$ & $\begin{array}{l}\text { public and private transport providers } \\
\text { and other service companies, } \\
\text { multinational companies as well as } \\
\text { SMEs (both buyers and suppliers (of } \\
\text { equipment for instance)), public } \\
\text { research centres, and universities. }\end{array}$ & $\begin{array}{l}\text { no rules concerning IPR that are } \\
\text { specific to PREDIT, IPR regulations } \\
\text { vary according to the type of } \\
\text { financing and the financing } \\
\text { organisation. Generally, results of } \\
\text { projects that have received subsidies } \\
\text { or refundable loans belong to the } \\
\text { participants, }\end{array}$ \\
\hline $\mathrm{NL}$ & TS & $\begin{array}{l}\text { TS is composed of a generic R\&D } \\
\text { component and a complementary } \\
\text { measure to support specific areas } \\
\text { where international co-operation is } \\
\text { deemed to be of strategic importance } \\
\text { to the Netherlands. These include } \\
\text { grants to support EUREKA projects, } \\
\text { market research and cooperative } \\
\text { projects to support engagement with } \\
\text { emerging markets, co-operation } \\
\text { within the maritime sector and co- } \\
\text { operation in the area of ICT- } \\
\text { breakthrough projects. In addition, TS } \\
\text { sponsors measures such as } \\
\text { networking, internationalisation and } \\
\text { knowledge transfer }\end{array}$ & $\begin{array}{l}\text { In order to be eligible for support the } \\
\text { application is submitted by one Dutch } \\
\text { firm in co-operation with one or } \\
\text { several companies and/or knowledge } \\
\text { institutes or universities. This may } \\
\text { involve co-operation with Dutch or } \\
\text { foreign partners. }\end{array}$ & $\begin{array}{l}\text { The IPR belongs to the consortium. It } \\
\text { is up to the partners to make the } \\
\text { specific arrangements. }\end{array}$ \\
\hline NO & TEFT & $\begin{array}{l}75 \% \text { of the total costs of the project } \\
\text { are funded by TEFT which is used to } \\
\text { buy services from the selected } \\
\text { research institution(s) or other } \\
\text { companies. The participating } \\
\text { company itself does not receive any } \\
\text { money directly from TEFT. }\end{array}$ & $\begin{array}{l}\text { co-operation takes place between the } \\
\text { SME and one or more research } \\
\text { institutes or other SMEs (national or } \\
\text { European). }\end{array}$ & $\begin{array}{l}\text { The rights on the results are defined } \\
\text { in the project contract and generally } \\
\text { belong to the SMEs. All rights to } \\
\text { results not explicitly described in the } \\
\text { contract belong to Sintef }\end{array}$ \\
\hline
\end{tabular}




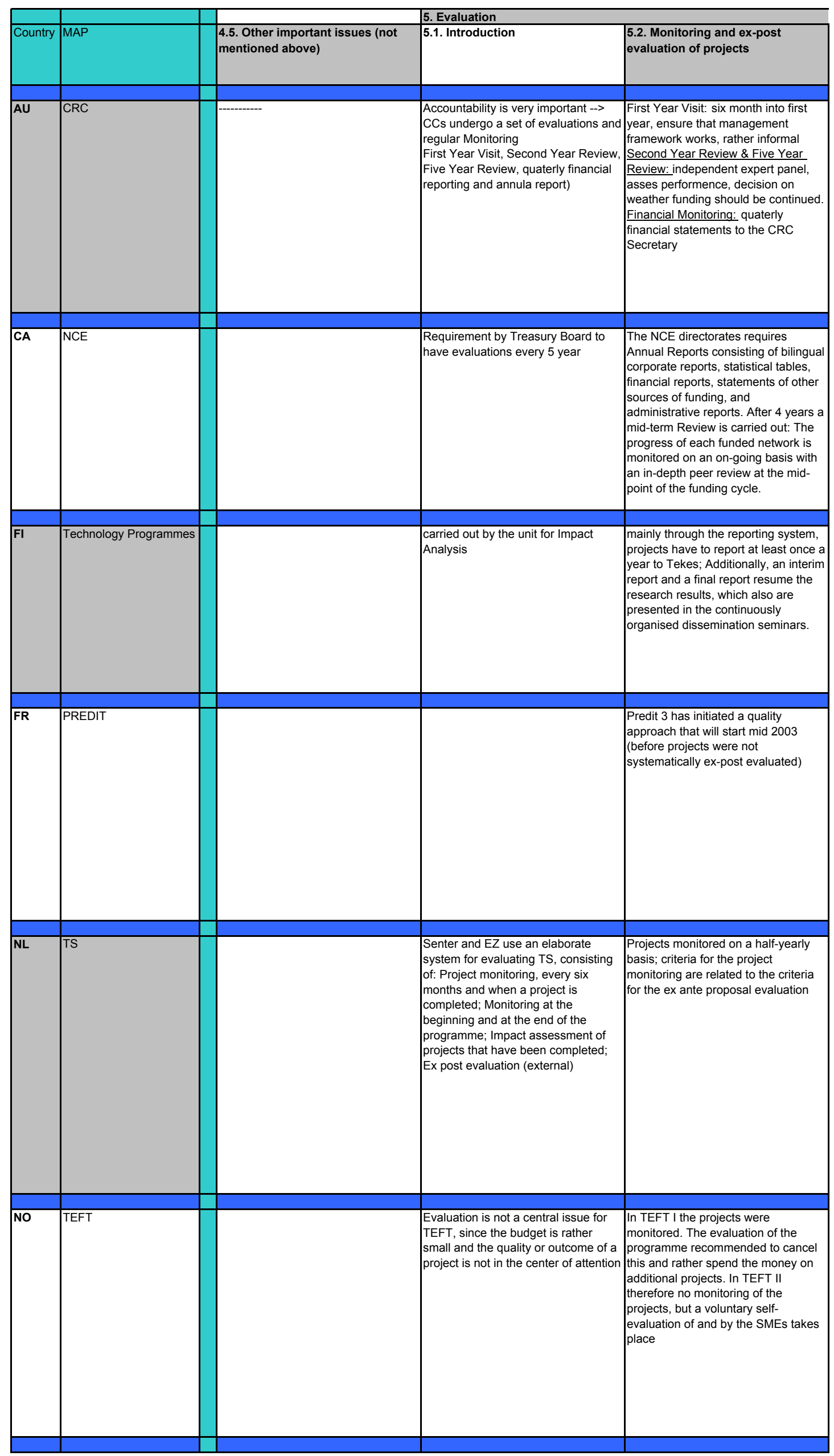




\begin{tabular}{|c|c|c|c|c|}
\hline Country & MAP & 5.3. Programme Evaluation & 5.4. Other important issues (not & \begin{tabular}{|l|} 
6. Horizontal Issues \\
6.1. Introduction
\end{tabular} \\
\hline & & & mentioned above) & \\
\hline AU & CRC & & spin off generation & $\begin{array}{l}\text { organisational learning } \\
\text { governance } \\
\text { institutional sttings } \\
\text { involvement of SMEs } \\
\text { cross/border cooperation } \\
\text { transferability of key elements } \\
\text { unintended effects,... }\end{array}$ \\
\hline CA & NCE & $\begin{array}{l}3 \text { program evaluations so far: } 1 \text { st on management } \\
\text { issues of NCEs; } 2 \text { nd on impacts of program; 3rd on } \\
\text { rationale of programme }\end{array}$ & 97 spinn-offs so far & \\
\hline FI & Technology Programmes & $\begin{array}{l}\text { Ex-ante evaluation of the programme: group support } \\
\text { system (GSS) to analyse prospective technological } \\
\text { advances and to take the 'lessons learned' from } \\
\text { earlier programmes into account for the planning of } \\
\text { a new programme. Monitoring of the programme: } \\
\text { steering group of projects; Mid-term evaluations; Ex- } \\
\text { post evaluations }\end{array}$ & $\begin{array}{l}\text { the responsibility of Tekes' } \\
\text { Evaluation and Quality Unit, which } \\
\text { also selects the external evaluators } \\
\text { (if needed) }\end{array}$ & $\begin{array}{l}\text { mid } 1990 \text { more thematic approaches were } \\
\text { introduced (e.g. environmental perspectives); } \\
\text { Recently, the function of technology programmes } \\
\text { concentrates on enhancing of the national } \\
\text { innovation system and field-specific innovation } \\
\text { environments for technology development, } \\
\text { transfer and implementation. The programme try } \\
\text { to stimulate networking and co-operation between } \\
\text { actors and to connect the Finnish context with } \\
\text { international trends and developments. }\end{array}$ \\
\hline FR & PREDIT & \begin{tabular}{|l|} 
Predit 1 (1990-1994): final ev. in 1995; Predit 2 \\
(1996-2000): mid-term in 1998, ex-post in 2001; \\
Predit 3 2002-2006): mid-term and ex-post foreseen
\end{tabular} & & \\
\hline NL & TS & \begin{tabular}{|l|} 
programme evaluation consists of 3 types: project \\
reporting system + annual impact assessment \\
conducted by Senter staff + every four years ex post \\
evaluation by external evaluators, which serves to \\
regulate the internal reviews deriving from the \\
project level evaluations
\end{tabular} & & \\
\hline NO & TEFT & \begin{tabular}{|l|} 
ex-post evaluation of TEFT II is currently in the \\
preparatory phase; The results should be available \\
by the 1 st of October 2003. evaluation addressed \\
mainly 4 issues: 1 . the programme from the \\
companies' perspective (what were the results and \\
impacts achieved?) 2 . the programme from the \\
perspective of the R\&D institute (what impact had \\
the programme participation?) 2 . the role of the \\
technology attachés (how did they do their work, did \\
they do it right? 3 . Networking (what effects had the \\
programme on network building of regional actors?)
\end{tabular} & & \\
\hline
\end{tabular}




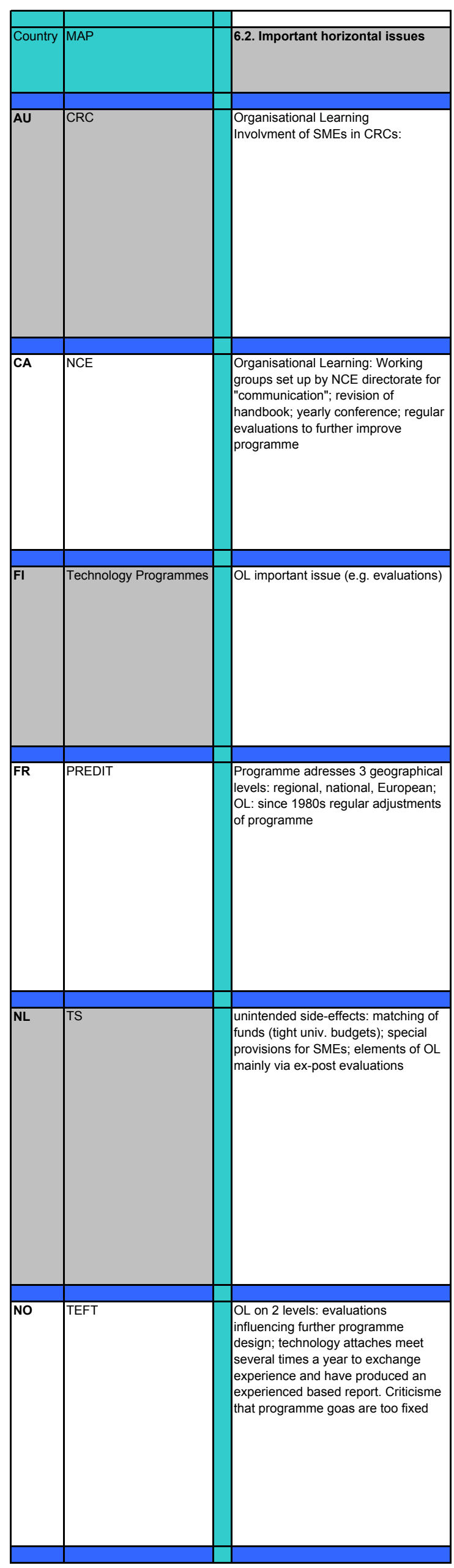




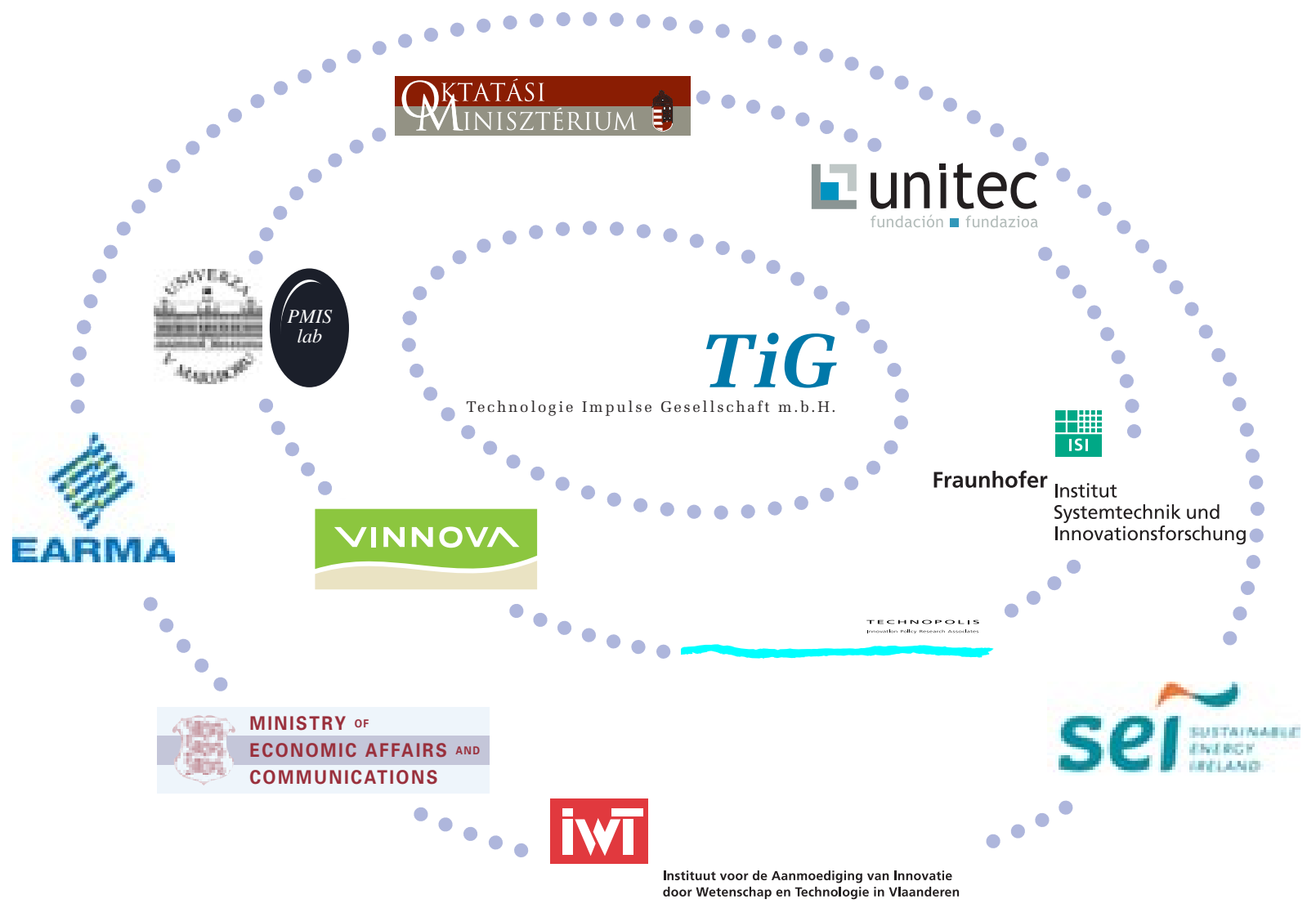

\section{Information / Contact:}

TIG Technologie Impulse Gesellschaft

Grillparzerstrasse 7

A-1010 Vienna, Austria

T: $+43 / 1 / 5132627-0$

F: $+43 / 1 / 5132627-10$

E: birgit.baumann@tig.or.at or barbara.kunz@tig.or.at

W: www.tig.or.at

Further information available on the MAP-TN homepage:

www.map-network.net 